\title{
Face In Video Evaluation (FIVE) Face Recognition of Non-Cooperative Subjects
}

Patrick Grother

George Quinn

Mei Ngan

This publication is available free of charge from:

https://doi.org/10.6028/NIST.IR.8173

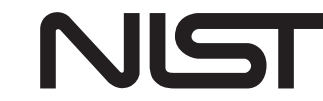

National Institute of Standards and Technology U.S. Department of Commerce 


\title{
Face In Video Evaluation (FIVE) Face Recognition of Non-Cooperative Subjects
}

\author{
Patrick Grother \\ George Quinn \\ Mei Ngan \\ Information Access Division \\ Information Technology Laboratory
}

This publication is available free of charge from:

https:/ /doi.org/10.6028/NIST.IR.8173

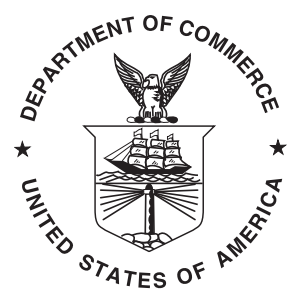

U.S. Department of Commerce Wilbur L. Ross, Jr., Secretary

National Institute of Standards and Technology Kent Rochford, Acting NIST Director and Under Secretary of Commerce for Standards and Technology 


\section{ACKNOWLEDGEMENTS}

The authors would like to thank the Department of Homeland Security (DHS) Science and Technology (S\&T) Directorate First Responders Group Office for Public Safety Research for support of this effort.

Additionally, we thank the DHS S\&T Homeland Security Advanced Research Agency Air Entry/Exit Reengineering (AEER) Directorate for their support.

We are indebted to the staff of the Pacific Northwest National Laboratory for their development and collection of imagery used in this study. Similarly, we are grateful to SAIC and the staff at the Maryland Test Facility for their development and collection of imagery used in this study.

Thanks also go to the Richard Lazarick, Michael Garris, Marek Rejman-Greene, Jason Prince, and Catherine Tilton for discussion and reviews of this material.

\section{DISCLAIMER}

Specific hardware and software products identified in this report were used in order to perform the evaluations described in this document. In no case does identification of any commercial product, trade name, or vendor, imply recommendation or endorsement by the National Institute of Standards and Technology, nor does it imply that the products and equipment identified are necessarily the best available for the purpose.

\section{STRUCTURE OF THIS REPORT}

The report is organized with an executive summary, a high-level background, and a technical summary preceeding the main body of the report which gives more detailed information on participation, test design, performance metrics, datasets, and the results.

\begin{tabular}{|l|l|l|l|}
\hline \multicolumn{4}{|c|}{ PARTICIPANT KEY } \\
\hline $\mathrm{A}=$ DIGITAL BARRIERS & $\mathrm{E}=$ NEUROTECHNOLOGY & $\mathrm{I}=$ EYEDEA & $\mathrm{M}=$ NEC \\
\hline $\mathrm{B}=$ HBINNO & F = VAPPLICA & $\mathrm{J}=$ HISIGN & $\mathrm{N}=$ TOSHIBA \\
\hline C = VIGILANT & G = MORPHO & $\mathrm{K}=$ COGNITEC & $\mathrm{Q}=$ IMAGUS \\
\hline $\mathrm{D}=$ AYONIX & $\mathrm{H}=$ 3M COGENT & $\mathrm{L}=$ CYBEREXTRUDER & $\mathrm{R}=$ RANK ONE \\
\hline
\end{tabular}

\begin{tabular}{|l|l|l|l|l|l|}
\hline SET & SCENE & CAMERA & SET & SCENE & CAMERA \\
\hline C & PHOTOJOURNALISM & PRO & T & CONCOURSE & PRO \\
\hline J & PASSENGER LOADING & PRO & H & CONCOURSE & PRO \\
\hline P & SPORTS ARENA & CONSUMER & U & CHOKEPOINT & WEBCAM \\
\hline L & LUGGAGE RACK & WEBCAM & & & \\
\hline
\end{tabular}




\section{EXECUTIVE SUMMARY}

$\triangleright$ Overview: This report documents the Face in Video Evaluation (FIVE), an independent, public test of face recognition of non-cooperating subjects who are recorded passively and are mostly oblivious to the presence of cameras. The report enumerates accuracy and speed of face recognition algorithms applied to the identification of persons appearing in video sequences drawn from six different video datasets mostly sequestered at NIST. These datasets represent video surveillance, chokepoint, and social media applications of the technology. In six cases, videos from fixed cameras are searched against portrait-style photographs of up to 48000 enrolled identities. In one case, videos are searched against faces enrolled from other videos. Additionally, the effect of supplementing enrollment with non-frontal images is examined.

$\triangleright$ Participation: FIVE was open to any organization worldwide, at no charge. The research arms of sixteen major commercial suppliers of face recognition technologies submitted thirty six algorithms, allowing FIVE to document a robust comparative evaluation. The algorithms were submitted to NIST in December 2015 so this report does not capture research and development gains since then. The algorithms are research prototypes, evaluated as black boxes without developer training or tuning. They implement a NIST-specified interface and so may not be immediately commercially available. They run without graphical processing units (GPU).

$\triangleright$ Difficulty: Face recognition is much more difficult in non-cooperative video than with traditional portrait-style photos. The initial face detection task is non-trivial because a scene may contain no faces or may contain many, and these can appear over a range of resolutions (scales), orientations (poses), and illumination conditions. Second, subjects move, so their faces must be tracked through time and this is harder when motion blur occurs or when a face is occluded by closer persons or objects. Third, resolution in video is compromised by optical tradeoffs (magnification, field of view, depth of field, cost) and then by compression used to satisfy data rate or storage limitations. Finally, other adverse aspects of image quality and face presentation degrade recognition scores so that scores from unrelated individuals can be similarly high, making discrimination between known and unknown individuals error prone. This leads to the possibility that a member of the public can be falsely matched to someone on a watchlist; the occurence of such hazards is mitigated by elevating a recognition threshold.

$\triangleright$ Key conclusions: This study was conducted to support new and existing applications of face recognition, particularly to assess viability and technology readiness. These range from surveillance, to expedited single-factor access control, and to the tagging of faces uploaded to social media. Whether face recognition can be used successfully depends on an operations-research analysis of the intended use given the most important design parameters in Table 1. This report provides data to inform that. For example, timing estimates imply that real-time processing on a single server is achievable only with certain algorithms.

This report documents situations where face recognition of non-cooperative persons is accurate enough to satisfy some operational requirements. It also demonstrates cases where the core recognition technology fails to compensate for deficient imaging. That notwithstanding, subjects appearing in video can be identified with error rates approaching those measured in cooperative face recognition, but only if image quality reaches the level attained in engineered systems such as e-Passport gates. That is a very difficult goal.

High accuracy recognition of passively-imaged subjects is only achievable with: a) a small minority of the algorithms tested here; b) a dedicated and deliberate design effort that must embed optical, architectural, human factors, operationsresearch, and face recognition expertise; c) galleries limited to small numbers of actively curated images; and d) field tests with empirical quantitative calibration and optimization.

None of the datasets used in this report represent peak attainable performance. Given better cameras, better design, and the latest algorithm developments, recognition accuracy can advance even further. However, even with perfect

\begin{tabular}{|l|l|l|l|}
\hline \multicolumn{5}{|c|}{ PARTICIPANT KEY } \\
\hline $\mathrm{A}=$ DIGITAL BARRIERS & $\mathrm{E}=$ NEUROTECHNOLOGY & $\mathrm{I}=$ EYEDEA & $\mathrm{M}=$ NEC \\
\hline $\mathrm{B}=$ HBINNO & $\mathrm{F}=$ VAPPLICA & $\mathrm{J}=$ HISIGN & $\mathrm{N}=$ TOSHIBA \\
\hline $\mathrm{C}=$ VIGILANT & $\mathrm{G}=$ MORPHO & $\mathrm{K}=$ COGNITEC & $\mathrm{Q}=$ IMAGUS \\
\hline $\mathrm{D}=$ AYONIX & $\mathrm{H}=$ 3M COGENT & $\mathrm{L}=$ CYBEREXTRUDER & $\mathrm{R}=$ RANK ONE \\
\hline
\end{tabular}

\begin{tabular}{|l|l|l|l|l|l|}
\hline SET & SCENE & CAMERA & SET & SCENE & CAMERA \\
\hline C & PHOTOJOURNALISM & PRO & T & CONCOURSE & PRO \\
\hline J & PASSENGER LOADING & PRO & H & CONCOURSE & PRO \\
\hline P & SPORTS ARENA & CONSUMER & U & CHOKEPOINT & WEBCAM \\
\hline L & LUGGAGE RACK & WEBCAM & & & \\
\hline
\end{tabular}


design, some proportion of a non-cooperative population will not be recognized simply because their faces were not availble to the receiving system. Such failure to acquire cases occur when subjects never look toward the camera (e.g. at a mobile phone instead) or because their faces were occluded (e.g. by sunglasses, hats, or by taller people standing in front of them). While such failures can be mitigated by careful placement of, in principle, multiple cameras, recognition essentially only succeeds if a clear line of sight to a sufficiently frontal face can be engineered (assuming the reference image is frontal).

Deployment should proceed only after quantitative assessment of objectives, alternatives, ease of evasion or circumvention, enrolled population sizes, search volumes, the proportion of searches expected to have an enrolled mate, accuracy requirements, consequences and procedures for resolution of errors, and speed and hardware cost constraints. In particular, deployers must weight their tolerance for misses and their risk appetite. In addition, when non-cooperative face recognition is used to identify individuals nominated to a watchlist, human reviewers must be employed to adjudicate whether candidate matches are true or false positives. This has two consequences. First, the recognition system must be dynamically configured to produce (true or false) candidate alerts at a rate matched to available labor resources. Second, because humans also commit face recognition errors, the overall error rates of the hybrid machine-human system must be understood and planned for.

\section{OUT OF SCOPE}

The following are out of the FIVE scope: One-to-one verification of identity claims; identification from body worn cameras, license plate cameras, drones and other aerial vehicles; video analytics, scene understanding, anomaly detection, and spatial boundary violation; suspicious behavior and intent detection; estimation of emotional state; full body recognition (static and kinematic) including gait recognition; use of imagery from exotic sensors such as infrared and thermal emission.

\begin{tabular}{|l|l|l|l|}
\hline \multicolumn{4}{|c|}{ PARTICIPANT KEY } \\
\hline $\mathrm{A}=$ DIGITAL BARRIERS & E = NEUROTECHNOLOGY & I = EYEDEA & $\mathrm{M}=$ NEC \\
\hline $\mathrm{B}=$ HBINNO & $\mathrm{F}=$ VAPPLICA & $\mathrm{J}=$ HISIGN & $\mathrm{N}=$ TOSHIBA \\
\hline $\mathrm{C}=$ VIGILANT & $\mathrm{G}=$ MORPHO & $\mathrm{K}=$ COGNITEC & $\mathrm{Q}=$ IMAGUS \\
\hline $\mathrm{D}=$ AYONIX & $\mathrm{H}=$ 3M COGENT & $\mathrm{L}=$ CYBEREXTRUDER & $\mathrm{R}=$ RANK ONE \\
\hline
\end{tabular}

\begin{tabular}{|l|l|l|l|l|l|}
\hline SET & SCENE & CAMERA & SET & SCENE & CAMERA \\
\hline C & PHOTOJOURNALISM & PRO & T & CONCOURSE & PRO \\
\hline J & PASSENGER LOADING & PRO & H & CONCOURSE & PRO \\
\hline P & SPORTS ARENA & CONSUMER & U & CHOKEPOINT & WEBCAM \\
\hline L & LUGGAGE RACK & WEBCAM & & & \\
\hline
\end{tabular}




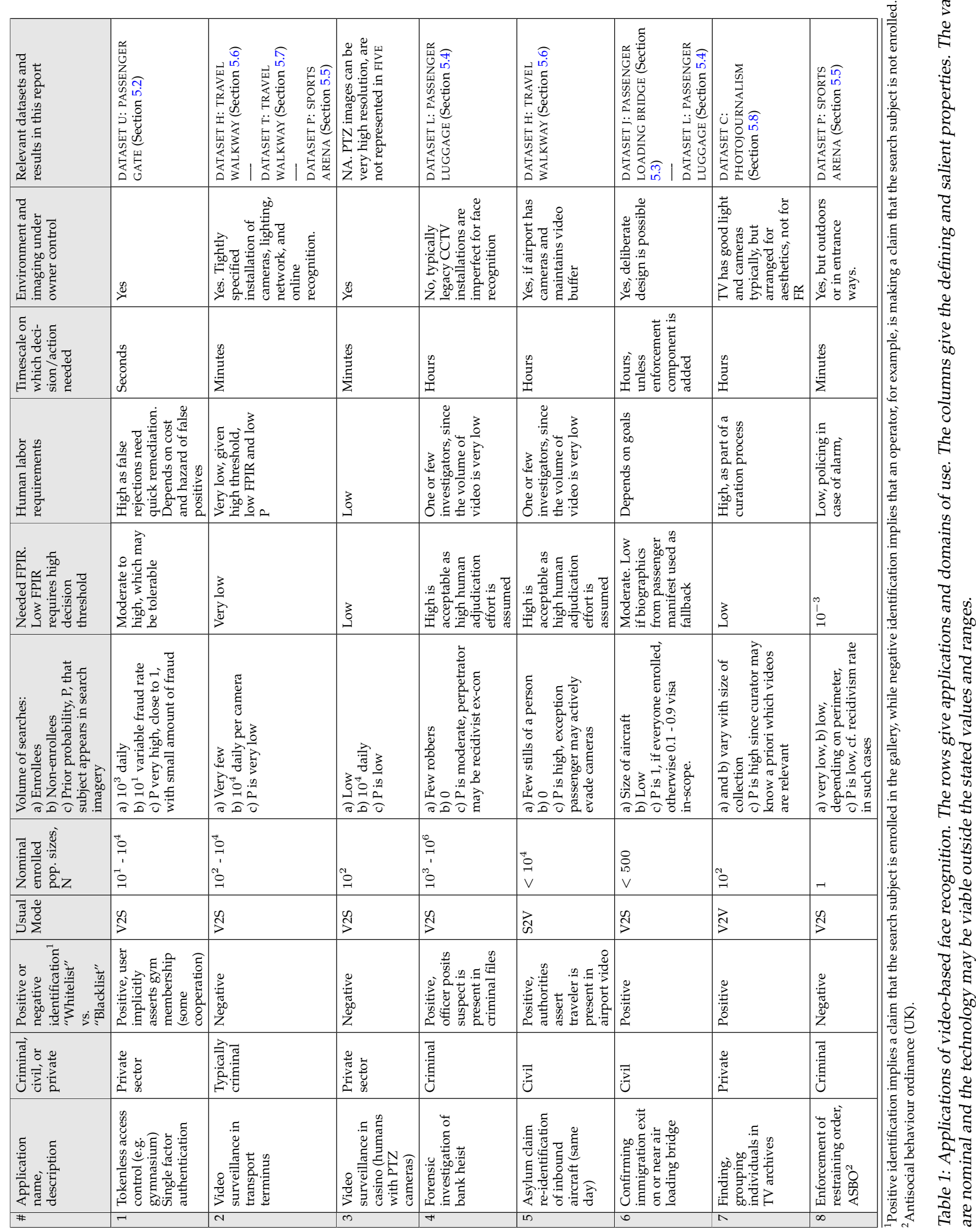




\section{BACKGROUND}

Non-cooperative face recognition: Prior NIST reports on the evaluation of face recognition prototypes [19, 21] have quantified the accuracy available from still photographs that were collected with cooperation of the subject and for which the imaging apparatus, environment and configuration were controlled according to formal face recognition standards $[15,18]$. In contrast, this report details recognition performance available from video sequences of human subjects who do not make explicitly cooperative attempts to present their faces to a camera; instead they are observed passively and are usually oblivious of the presence of the cameras. Non-cooperative imaging of subjects is generally adverse to recognition accuracy, because face recognition algorithms are sensitive to head orientation relative to the optical axis of the camera, accompanying shadows, and to variations in facial expression. Note also that cooperation is not all-or-nothing: It can be induced by using a video display attractor. We investigate this with Dataset J.

Challenges of face recognition in video imagery: Video sequences present additional challenges to face recognition systems. First, subjects may be entirely absent for extended periods, and with a cluttered background, algorithms will yield false detections. There is generally a tradeoff between missed detections and adequate suppression of false detections. Second, the subjects are generally moving, and this motion is not necessarily parallel to the optical axis. Motion, which can cause blur, requires algorithms to track faces through time, a task that is not trivial in cluttered scenes with variable lighting and the possibility of occlusion by other subjects. Third, there may be multiple subjects present, each of which must be localized. Moreover, this must occur over varying resolutions (scales) and head orientations (poses). Fourth, facial detail may be lost to motion blur and to the lossy compression that remains a ubiquitous requirement in video transmission and storage. Finally, optical distortion can occur when subjects are displaced off the optical axis, or, more rarely, are too close to the camera.

The FIVE study leverages seven video datasets whose characteristics differ, with variations in duration, frame rate, camera quality, camera configuration, illumination environment and geometric placement - see Table 3. These factors are known to influence accuracy, and sometimes computational cost. Note however, that all of the cameras used in the collection had fixed position, orientation and optics, and none were under active human control. Even without variation of those parameters, face recognition in video presents considerable challanges beyond those faced in traditional still-image tasks.

Applications: Non-cooperative face recognition can be applied in a number of applications, beyond the video surveillance task popularized in television and films. These can be categorized in several ways: First, many applications search video against enrolled still images (V2S), others could search still to video (S2V), and yet more might search video to video $(\mathrm{V} 2 \mathrm{~V})$. This will have architectural and computational implications. Second, they can be categorized by whether the technical specifications (camera, optics, illumination) are under the control of the operator vs. provided to a receiving agency, as is. Third, applications vary by the frequency with which enrolled subjects are encountered in a search. Fourth is whether they embed positive or negative identity claims - would a typical user claim to be enrolled, or claim not to be enrolled. The canonical application, video surveillance, involves searching live video feeds against enrollment imagery from known persons of interest. This "blacklist" search is a negative identification application in that there is an implicit claim that most searches are not expected to have an enrolled mate. This prior probability that an enrolled subject is actually encountered can be very low. If it is too low, then this contraindicates deployment of the system, unless the risk of not doing so is to miss a person of such interest that it has large adverse effects. An additional trouble with "rare hit" applications is boredom and lack of reward for the human reviewers who adjudicate candidate matches.

The report also seeks to support positive "whitelist" identification applications such as expedited access control in which non-cooperative subjects are not required to make a deliberate interaction with a biometric sensor, such that the duration of the transaction is by-definition zero. This is contrary to the more typical access control application in which a subject presents a credential to a reader (swipe or chip read, for example), makes one or more cooperative presentations to

\begin{tabular}{|l|l|l|l|}
\hline \multicolumn{4}{|c|}{ PARTICIPANT KEY } \\
\hline A = DIGITAL BARRIERS & E = NEUROTECHNOLOGY & I = EYEDEA & M = NEC \\
\hline B = HBINNO & F = VAPPLICA & J = HISIGN & N = TOSHIBA \\
\hline C = VIGILANT & G = MORPHO & K = COGNITEC & $\mathrm{Q}=$ IMAGUS \\
\hline D = AYONIX & $\mathrm{H}=$ 3M COGENT & L = CYBEREXTRUDER & $\mathrm{R}=$ RANK ONE \\
\hline
\end{tabular}

\begin{tabular}{|l|l|l|l|l|l|}
\hline SET & SCENE & CAMERA & SET & SCENE & CAMERA \\
\hline C & PHOTOJOURNALISM & PRO & T & CONCOURSE & PRO \\
\hline J & PASSENGER LOADING & PRO & H & CONCOURSE & PRO \\
\hline P & SPORTS ARENA & CONSUMER & U & CHOKEPOINT & WEBCAM \\
\hline L & LUGGAGE RACK & WEBCAM & & & \\
\hline
\end{tabular}


a sensor (finger placement, for example), and then proceeds. In contrast, passive non-cooperative imaging elides the possession and presentation of a credential. The downside to this approach is the likely reduction in true acceptance rates associated with configuring the system to limit false positives inherent in comparing imagery with that of $\mathrm{N}$ enrolled identities.

Note that in some applications, the enrolled subjects may be entirely unknown i.e. there may be no biographic metadata - and the goal of biometric search is simply to link images across two or more events, without any actual identification.

Table 1 gives a matrix of applications and their defining properties. This is intended to support prospective users in analysis of their application and mapping that onto results available later in this report.

Evasion of face recognition: This report does not cover a further operational challenge for recognition: subjects who actively impede face processing. Deliberately uncooperative subjects can expend arbitrary effort to evade detection and recognition by avoiding the camera, either completely or by keeping their head down, or by altering the appearance of their face, for example by wearing (sun)glasses [32], hats, cosmetics, and masks. The likelihood that such techniques will be used - they have been ubiquitous in bank robberies since the advent of the CCTV camera - may well contraindicate the use of face recognition. Such techniques can be 100\% effective. Effective evasion is however predicated on knowledge of when cameras are present and face recognition may be in use. Without that knowledge, successful evasion would require continuous persistent effort.

State of face recognition research: While face recognition has been a perenial popular research challenge since its inception, there has been a marked escalation in this research in recent years due to the confluence of several supporting factors. These are the development of convolutional neural networks and deep learning [27] schemes, particularly for face recognition [30], which invariably leverage [31] the ever increasing amount of web-downloadable face images. The face imagery is taken from identity-labeled photographs downloaded automatically from social media. These are available in large part due to the advent of the digital camera, particularly on the smart phone, and critically, the internet as a distribution mechanism.

Open-universe identification: Non-cooperative face recognition is implicitly a one-to-many identification task. It is not a one-to-one verification task because it does not involve the subject in making an identity claim. As such, it requires unknown video imagery to be compared with that previously collected from multiple individuals. Given $\mathrm{N}$ individuals, the task is called 1-to-N identification, the goal being to determine the identity of the query subject. However, a key point is that most applications are open universe where some proportion of query subjects will not have a corresponding mate entry among the previously enrolled identities. The face identification algorithm must then minimize both false positive errors where an unenrolled person is mistakenly returned, and a false negative error where an enrolled person is missed. This is critical whenever the proportion of non-mated searches is naturally large particularly in the canonical "watchlist" surveillance application where a large majority of individuals in the field of view are not enrolled and should not be returned by the system. So a face recognition system looking for terrorism suspects in a crowded railway station must be configured to produce few false positives, no more than what can be sustainably adjudicated by trained reviewers who would determine the veracity of the candidate match, and then initiate action. If, on the other hand, the proportion of unmated searches is low, as is the case, for example, with patrons entering their gymnasium, the system must be configured to tolerate a few false positives, i.e. to admit the infrequent non-customer who attempts access.

Relevant accuracy measures: It is necessary to report accuracy in terms of both false negative identification rate quantifying how often enrollees are not recognized, and the false positive identification rate stating how often algorithms incorrectly issue false alarms. Accordingly, FIVE supported measurement of open-set accuracy, including both actor false negatives ("misses") and non-actor false positives ("false alarms"). In this respect, this benchmark differs from recent face recognition evaluations [25] that assume all searches have an enrolled mate.

Academic research focuses on closed-universe applications: Most research papers on face identification have two

\begin{tabular}{|l|l|l|l|}
\hline \multicolumn{5}{|c|}{ PARTICIPANT KEY } \\
\hline $\mathrm{A}=$ DIGITAL BARRIERS & $\mathrm{E}=$ NEUROTECHNOLOGY & $\mathrm{I}=$ EYEDEA & $\mathrm{M}=$ NEC \\
\hline $\mathrm{B}=$ HBINNO & $\mathrm{F}=$ VAPPLICA & $\mathrm{J}=$ HISIGN & $\mathrm{N}=$ TOSHIBA \\
\hline $\mathrm{C}=$ VIGILANT & $\mathrm{G}=$ MORPHO & $\mathrm{K}=$ COGNITEC & $\mathrm{Q}=$ IMAGUS \\
\hline $\mathrm{D}=$ AYONIX & $\mathrm{H}=$ 3M COGENT & $\mathrm{L}=$ CYBEREXTRUDER & $\mathrm{R}=$ RANK ONE \\
\hline
\end{tabular}

\begin{tabular}{|l|l|l|l|l|l|}
\hline SET & SCENE & CAMERA & SET & SCENE & CAMERA \\
\hline C & PHOTOJOURNALISM & PRO & T & CONCOURSE & PRO \\
\hline J & PASSENGER LOADING & PRO & H & CONCOURSE & PRO \\
\hline P & SPORTS ARENA & CONSUMER & U & CHOKEPOINT & WEBCAM \\
\hline L & LUGGAGE RACK & WEBCAM & & & \\
\hline
\end{tabular}


related limitations. First, their experiments do not include searches for which a mate does not exist in the enrolled gallery. This is a closed universe application of biometrics; it derives from the approach used in the computer vision and pattern recognition community in which benchmarks involve the classification of the image of an object to a closed set of exemplar objects. This almost always does not apply in biometrics, because some subjects have usually not been encountered before. For example, in a criminal justice application, the closed universe assumption is tantamount to assuming everyone has been previously arrested and photographed. By failing to execute non-mate searches, academic experimentation does not measure false positive identification rates i.e. proportion of non-mate searches that incorrectly return an enrolled identity. Instead, most research reports accuracy using rank based recognition rates drawn from the cumulative match characteristic. While some publications [31] do execute 1:1 verification, with many impostor comparisons, they do not explicitly consider the largest of $\mathrm{N}$ impostor scores generated in a 1:N search. It is this extreme value which, if high enough, leads to false alarms in identification systems ${ }^{1}$.

The underlying point here is that much research is directed at optimizing a metric that is often inappropriate to typical use cases. Specifically, the research evalutions assume the prior probability that a search photograph has a mate is 1 . This departs from operational reality in that the prior probability can span many orders of magnitude below 1 . At the high end, for example, a majority of faces uploaded to a face recognition tagging service on a social networking website will belong to the universe of friends of the uploader. In law enforcement applications, the prior probability that a photograph searched against a criminal database would have an enrolled mate will be related to the recidivism rate which has been measured around 0.6 in the United States [10]. In a casino, the proportion of card-sharps among the gambling population might never exceed 1:1000, while in a border crossing drug interdiction setting the prior probability could be below 1:10000. In a counter-terrorism application, the probability could be much lower still. The point is that for all the unenrolled subjects, the face recognition system must correctly not issue a false alarm, or, least do so rarely. This necessitates good algorithmic discrimination and high, stringent, similarity thresholds. This is at odds with much of the academic research which focuses on employing deep convolutional neural networks (DCNNs) designed for their invariance to pose, translation, illumination. That is appropriate in a social media setting perhaps. It is less so for governmental applications where, often, the camera and illumination can be controlled even with a non-cooperating population. There remain open questions with DCNNs: Can they surpass traditional approaches at very low false positive rates, at least in cooperative portrait-style imagery? Can they be used "off-the-shelf", i.e. without either training from scratch, or without some kind of adaptation e.g. transfer learning.

Human adjudication of true and false matches: Most applications of automated face recogition are monitored by human operators who adjudicate recognition decisions. So, for example, it is typical for sets of five e-Passport verification gates to be observed by an immigration officer who can open the gate if a traveler (who may be a legimate passport holder or an impostor) is not authenticated automatically, or refer him to a traditional process. In one-to-many situations, for example, the detection of duplicate visa or driving license submissions, the examining official must determine whether the identity of the search image and the hypothesized gallery entry are indeed the same, or if a false positive has occured. The system managers must ensure sufficient labor is available to adjudicate such outcomes. Practically, the recognition threshold is set to limit the number to manageable levels.

\footnotetext{
${ }^{1}$ For quick background see http://www.wjscheirer.com/misc/openset/cvpr2016-open-set-part2.pdf, or [22] for the effect of extreme values on 1:N accuracy.

\begin{tabular}{|l|l|l|l|}
\hline \multicolumn{4}{|c|}{ PARTICIPANT KEY } \\
\hline A = DIGITAL BARRIERS & E = NEUROTECHNOLOGY & I = EYEDEA & M = NEC \\
\hline B = HBINNO & F = VAPPLICA & J = HISIGN & N = TOSHIBA \\
\hline C = VIGILANT & G = MORPHO & K = COGNITEC & Q = IMAGUS \\
\hline D = AYONIX & H = 3M COGENT & L = CYBEREXTRUDER & R = RANK ONE \\
\hline
\end{tabular}

\begin{tabular}{|l|l|l|l|l|l|}
\hline SET & SCENE & CAMERA & SET & SCENE & CAMERA \\
\hline C & PHOTOJOURNALISM & PRO & T & CONCOURSE & PRO \\
\hline J & PASSENGER LOADING & PRO & H & CONCOURSE & PRO \\
\hline P & SPORTS ARENA & CONSUMER & U & CHOKEPOINT & WEBCAM \\
\hline L & LUGGAGE RACK & WEBCAM & & & \\
\hline
\end{tabular}
}




\section{TECHNICAL SUMMARY}

Face detection: The first step in automated identification in video sequences is face detection. This is challenging due to variation in resolution and head orientation (pose). Given the same input video clips, algorithms vary by an order of magnitude in the number of faces they detect. Variation arises due to false detections (of non-faces), missed detections (of actual faces), and fragmented tracking through time. The result is that in the highest quality dataset we ran $(\mathrm{H})$, using over 20 hours of video recorded in a transport terminus, the number of detections varied from 8 to 150 per minute, with a consensus (from the more accurate algorithms) of between 10 and 15 per minute - the actual number is not known, nor well defined. Similarly in 43 hours of video shot using 11 cameras mounted in the access areas of a sports arena (Dataset P), the number of detections also varies by a factor of 20 .

See Tables 19 and 24

Verbose detection algorithms - those that report more faces in video - cause face recognition implementations to incur computational expenses associated with searching their outputs. Their accuracy is not necessarily worse, becoming so only if the higher volume of detections yields more false positives, and that occurs only if non-faces, small faces or otherwise poor quality faces yield high impostor scores. Terse algorithms, those that produce so few detections that they miss some actual faces can clearly give increased false negative identification rates. We note a few instances of algorithms reporting fewer detections than there are actors.

See Figure 22

Variation across algorithms: As with most other tests of biometric recognition algorithms, there is a massive variation in accuracy between algorithms: some give many fewer errors than others. This is particularly pronounced here as noncooperative video stresses algorithms. For even the easiest dataset, with small $(N=480)$ galleries, the proportion of searches that do not yield the correct identity at rank $1^{2}$ ranges from below $1 \%$ to above $40 \%$.

See Table 6.

Similarly, using professional-grade ceiling-mounted cameras, miss rates can range from $20 \%$ to over $90 \%$ even for algorithms provided by experienced developers. While accuracy is just one factor necessary to successful deployment others are speed, scalability, stability, ease of integration and configuration, and cost - such results imply a "procurement risk" associated with fielding the wrong algorithm.

See Table 24.

Variation across datasets: The FIVE activity employed seven datasets representative of applications given at the foot of this page. These range from high quality datasets typical of what would be expected if a dedicated design and installation effort had been made, to a dataset more representative of many legacy installations where CCTV is available at a crime scene but is poorly-suited to face recognition. Dataset $C$ is difficult because it is comprised of celebrity videos, where high yaw angles are typical, and the enrollment images are also unconstrained. All other datasets used controlled portraits. The inset table shows rank one miss rates from five of the more accurate providers. The enrolled population size is small, $N=480$. These

\begin{tabular}{|l|r|r|r|r|r|r|r|}
\hline Alg $\backslash$ Dataset & U & J & L & P & H & T & C \\
\hline M30V & $1 \%$ & $6 \%$ & $12 \%$ & $14 \%$ & $15 \%$ & $13 \%$ & $42 \%$ \\
\hline I30V & $8 \%$ & $10 \%$ & $24 \%$ & $23 \%$ & $15 \%$ & $10 \%$ & $49 \%$ \\
\hline H30V & $4 \%$ & $12 \%$ & $25 \%$ & $39 \%$ & $16 \%$ & $16 \%$ & $60 \%$ \\
\hline N33V & $4 \%$ & $14 \%$ & $32 \%$ & $44 \%$ & $17 \%$ & $28 \%$ & $53 \%$ \\
\hline G32V $/$ G30V $^{+}$ & $6 \%$ & $+30 \%$ & $40 \%$ & $35 \%$ & $+21 \%$ & $23 \%$ & $36 \%$ \\
\hline
\end{tabular}

numbers are applicable to investigational applications where ranked candidate lists are examined by reviewers, without any thresholding. Miss rates range widely, and certain algorithms are ill-suited to certain datasets. For example, while several algorithms perform well on the professional surveillance Datasets $\mathrm{H}$ and $\mathrm{T}$, only one handles the lower resolution Datasets L and P, yielding about half as many errors as algorithms from the next most accurate developer.

We summarize accuracy by stating the best single camera results here. Later we report results for fusion across cameras.

Surveillance: When using ceiling-mounted professional-grade cameras to identify previously enrolled actors appearing in about 20 hours of transport-terminus surveillance video, the most accurate algorithm detects and reports more than 17000 faces, some of those actors, most of them un-enrolled passers-by. Of the actors, the algorithm incorrectly

\footnotetext{
${ }^{2}$ This report universally uses "miss rates" i.e. false negative identification error rates, $x$, where smaller values are better. It is more common to see "hit rate" values, $1-x$. We do not quote these because they can lead to a framing bias when success rates are high. For example, $97 \%$ and $99 \%$ correspond to three times as many errors.
}

\begin{tabular}{|l|l|l|l|}
\hline \multicolumn{5}{|c|}{ PARTICIPANT KEY } \\
\hline $\mathrm{A}=$ DIGITAL BARRIERS & $\mathrm{E}=$ NEUROTECHNOLOGY & $\mathrm{I}=$ EYEDEA & $\mathrm{M}=$ NEC \\
\hline $\mathrm{B}=$ HBINNO & $\mathrm{F}=$ VAPPLICA & $\mathrm{J}=$ HISIGN & $\mathrm{N}=$ TOSHIBA \\
\hline $\mathrm{C}=$ VIGILANT & $\mathrm{G}=$ MORPHO & $\mathrm{K}=$ COGNITEC & $\mathrm{Q}=$ IMAGUS \\
\hline $\mathrm{D}=$ AYONIX & $\mathrm{H}=3 \mathrm{M}$ COGENT & $\mathrm{L}=$ CYBEREXTRUDER & $\mathrm{R}=$ RANK ONE \\
\hline
\end{tabular}

\begin{tabular}{|l|l|l|l|l|l|}
\hline SET & SCENE & CAMERA & SET & SCENE & CAMERA \\
\hline C & PHOTOJOURNALISM & PRO & T & CONCOURSE & PRO \\
\hline J & PASSENGER LOADING & PRO & H & CONCOURSE & PRO \\
\hline P & SPORTS ARENA & CONSUMER & U & CHOKEPOINT & WEBCAM \\
\hline L & LUGGAGE RACK & WEBCAM & & & \\
\hline
\end{tabular}


misses about $20 \%$ of them in a gallery constructed from $\mathrm{N}=480$ individuals' frontal photographs. This degrades with population size as shown in the inset table. This applies to identification mode where a high threshold is set to limit false positives to just 10; this is a very low count given the 20 hour, 2 million frame,

\begin{tabular}{|l|r|r|r|}
\hline Dataset $\mathrm{H}$, Algorithm M30V & $\mathrm{N}=480$ & $\mathrm{~N}=4800$ & $\mathrm{~N}=48000$ \\
\hline Identification miss rate & $20 \%$ & $21 \%$ & $35 \%$ \\
\hline Investigation miss rate & $14 \%$ & $16 \%$ & $26 \%$ \\
\hline
\end{tabular}
duration of video and the presence of thousands individuals. If, instead, forensic investigators had isolated a clip of interest from the same video collection, say resulting from a terrorism incident at a particular time, then they could review candidate identities reported by face algorithms. In such cases, the recognition threshold is set to zero, and trained human reviewers adjudicate photos on candidate lists. As illustrated in the second row, rank-based identification miss rates are better because they allow weak-scoring hits to count. Elevating a threshold, on the other hand, causes some hits to become misses, but is necessary in most practical cases to limit false positives. Further gains are possible considering longer candidate lists. Here for a dataset of $\mathrm{N}=48000$ persons, the two most accurate algorithms would miss about $24 \%$ of suspects within the top-20 ranks vs. $26 \%$ at rank 1 .

See Tables $23-25$.

For this reason, it is imperative that enrolled population sizes be kept as small as possible as part of an active gallery curation process. The high accuracy values reported later are achieved with gallery sizes three order of magnitude below those used in contemporary cooperative passport and driving license de-duplication systems.

Boarding gate: In the case where a turnstile or gate is equipped with a camera that passively records subjects presenting paper barcode tokens, the most accurate algorithm will miss just $6 \%$ of subjects present in an enrolled database of $N=480$ individuals. This degrades as shown in the inset table. This benign approximately logarithmic increase in miss rates with database size has been reported in prior tests of face recognition with

\begin{tabular}{|l|r|r|r|}
\hline Dataset U: Gate & $\mathrm{N}=480$ & $\mathrm{~N}=4800$ & $\mathrm{~N}=48000$ \\
\hline Identification miss rate & $6 \%$ & $9 \%$ & $18 \%$ \\
\hline Investigation miss rate & $1 \%$ & $2 \%$ & $4 \%$ \\
\hline
\end{tabular}
cooperative portrait-style images [23], and is the underlying reason why face recognition has utility for large scale face identification. Accuracy may be improved with refined camera placement or additional cameras. See Tables 6 - 8 .

Aircraft loading bridge: Consider the use of ceiling mounted cameras deployed on the passenger loading bridge. The video imagery could be searched simultaneously against "blacklist" and "whitelist" galleries. In the former case the application would be to prevent certain individuals from boarding, essentially as a public safety or law enforcement (LE) matter. In the latter, there are at least two possible applications. One would be simple access control - to allow only ticketholders to board - where some gate mechanism would deny boarding to a traveler whose video imagery did not match the enrollment photo. A second whitelist application would be immigration exit - a facilitation application - in which the goal is for visa holders to confirm exit from a country by achieving a positive match against their enrollment record. This differs from the access control situation in terms of security goals and error consequences, and relevant performance metrics as shown in the inset table. Using a purpose-built simulated aircraft passenger loading bridge, the most accurate algorithm correctly identifies all but $6 \%$ of subjects when searching a gallery of $\mathrm{N}=480$ individuals. This is achieved with the aid of an attractor to induce a frontal

\begin{tabular}{|l|l|l|l|l|l|l|}
\hline Application & $\begin{array}{l}\text { Blacklist / } \\
\text { Whitelist }\end{array}$ & $\mathrm{N}$ & $\begin{array}{l}\text { Prob } \\
\text { Mate }\end{array}$ & $\begin{array}{l}\text { FPIR } \\
\text { Required }\end{array}$ & $\begin{array}{l}\text { False positive } \\
\text { Consequence }\end{array}$ & $\begin{array}{l}\text { False negative } \\
\text { Consequence }\end{array}$ \\
\hline Public safety LE & Black & $\mathrm{O}\left(10^{4}\right)$ & $\rightarrow 0$ & Very low & Detain innocent & Safety, crim. evasion \\
\hline Access control & White & $<500$ & $\rightarrow 1$ & 0.001 cf. eGate & Wrong boarding & Deny boarding \\
\hline Immigration exit & White & $<500$ & $\mathrm{P}($ visa $)$ & Low & $\begin{array}{l}\text { Overstay fraud } \\
\text { or wrong status }\end{array}$ & $\begin{array}{l}\text { Wrong immi. status } \\
\text { rest on biographic }\end{array}$ \\
\hline
\end{tabular}
view and a bottleneck to force a delay, and when the threshold is set to allow 10 false positives. In a video surveillance application intended to detect rare "blacklisted" individuals this number of false positives may be tenable during the boarding of individuals onto an aircraft, as officers could review those ten candidate hits. However, for the "whitelist" access control application, where positive match above threshold opens a gate, the threshold is probably too low because, from a security perspective, 10 false positives during the boarding of a 480 person aircraft is poor: the implied false positive rate $(\sim 0.02)$ is much higher than is typically configured in an access control system $(\leq 0.001)$. Absent fraud, all persons should legitimately be able to board an aircraft (by matching any enrolled identity), any misses would

\begin{tabular}{|l|l|l|l|}
\hline \multicolumn{4}{|c|}{ PARTICIPANT KEY } \\
\hline A = DIGITAL BARRIERS & E = NEUROTECHNOLOGY & I = EYEDEA & M = NEC \\
\hline B = HBINNO & F = VAPPLICA & J = HISIGN & N = TOSHIBA \\
\hline C = VIGILANT & G = MORPHO & K = COGNITEC & $\mathrm{Q}=$ IMAGUS \\
\hline D = AYONIX & $\mathrm{H}=$ 3M COGENT & L = CYBEREXTRUDER & $\mathrm{R}=$ RANK ONE \\
\hline
\end{tabular}

\begin{tabular}{|l|l|l|l|l|l|}
\hline SET & SCENE & CAMERA & SET & SCENE & CAMERA \\
\hline C & PHOTOJOURNALISM & PRO & T & CONCOURSE & PRO \\
\hline J & PASSENGER LOADING & PRO & H & CONCOURSE & PRO \\
\hline P & SPORTS ARENA & CONSUMER & U & CHOKEPOINT & WEBCAM \\
\hline L & LUGGAGE RACK & WEBCAM & & & \\
\hline
\end{tabular}


require human assistance and that would delay boarding. Now consider the immigration exit alternative where no gate is present, everyone proceeds regardless of the recognition results. A "miss" will later be met with an assertion from the traveller that he did indeed leave, which will be supported by biographic logs from passenger manifests. However, any visa overstayer could send a confederate, ensure that their face was missed (by looking down at a mobile phone). The overstayer could later leave and re-enter relying on that biographic claim.

See Figures 17 and 18.

Legacy activity monitoring cameras: We regard the webcam imagery of Dataset $\mathrm{L}$ as more representative of the sub-optimal imagery acquired in, for example, banks and convenience stores where the goal was recording but not specifically face recognition. The imagery is characterized by adverse elevation angles and lower resolution. In this case the $\mathrm{M}$ algorithms give fewer than half the errors of any other algorithm.

Effect of camera height: The algorithm effect is prominent for recognition when cameras are mounted above head height. Such installation is typical. For subjects in a concession stand queue of a sports arena, recognition accuracy in a gallery $N=480$ frontal stills is superior for cameras mounted at a height of 1.83 vs. 2.44 meters ( 6 vs 8 feet). However, the magnitude of this depends strongly on the algorithm - see the miss rates in the inset table for algorithms from the more accurate developers on this dataset.

\begin{tabular}{|l|r|r|r|r|r|}
\hline Miss rate & $\mathrm{G}$ & $\mathrm{M}$ & $\mathrm{H}$ & $\mathrm{I}$ & $\mathrm{R}$ \\
\hline High mount & $47 \%$ & $15 \%$ & $63 \%$ & $39 \%$ & $55 \%$ \\
\hline Low mount & $42 \%$ & $13 \%$ & $43 \%$ & $32 \%$ & $43 \%$ \\
\hline
\end{tabular}

Error rate tradeoff: As in all biometrics systems, false negative outcomes can be traded off against false positives. When a stringent high threshold is imposed to suppress false positives, the false negative rate rises. When false positives are tolerable, lower false negative rates are achievable. In conventional biometrics, it is ubiquitous to compute an error tradeoff characteristic and plot false negative vs. false positive identification rates. In surveillance, the actual number of subjects appearing within the field of view is unknown (and poorly defined) such that the false positive identification rate is replaced by the false positive count. In operations, the relevant metric becomes the false positive count per unit time given some canonical population flow rate.

See Figure 10.

In any case, both kinds of identity error are influential. Prospective deployers should consider the consequences of both types of error, and ensure (including contractually) that they have an ability to alter the threshold.

Algorithms for investigation vs. identification: It appears that some FIVE developers have not targeted recognition accuracy in cases where high thresholds are necessary to suppress false positives. Instead, as with much academic research, the focus has been on rank-based reporting. This manifests itself with high-threshold accuracy being far inferior to rank-based zero-threshold accuracy. Thus algorithms developed for investigational applications with low search volume (e.g. bank robberies) are not automatically appropriate to identification applications such as surveillance with crowds or sustained volumes.

Effect of enrolling multiple images per person: As face recognition has a well known dependence on head orientation, accuracy can be improved if a gallery is populated with additional views of a subject. Here, by enrolling three views together - full frontal and two images for which head yaw is $\pm 22^{\circ}$ - we see accuracy benefits with many algorithms. Modest gains are available in surveillance like applications, but more substantial gains appear for investigations where humans will review candidate lists. The inset table shows the proportion of actors not returned among the top 10 candidates. The technique is effective at promoting weak-scoring identity hypotheses up the candidate list. The technique has implications for standardized photo capture. While the face recognition industry has grown around the collection and enrollment of

\begin{tabular}{|l|r|r|r|r|}
\hline Enrollment & M & I & G & H \\
\hline One view & $4 \%$ & $5 \%$ & $19 \%$ & $18 \%$ \\
\hline Three views & $3 \%$ & $4 \%$ & $10 \%$ & $9 \%$ \\
\hline
\end{tabular}
frontal portrait photographs, supported by formal standards, the forensics community, motivated by their involvement in the adjudication of outputs of automated face recognition systems, has called for a) higher resolution photographs, and b) additional views. Greater resolution is available with virtually all cameras today. Collection of photographs from different viewpoints will likely require additional labor and possibly equipment, so further standardization should be predicated on an understanding of which views are most beneficial, the consistency with which they can be achieved,

\begin{tabular}{|l|l|l|l|}
\hline \multicolumn{5}{|c|}{ PARTICIPANT KEY } \\
\hline $\mathrm{A}=$ DIGITAL BARRIERS & $\mathrm{E}=$ NEUROTECHNOLOGY & $\mathrm{I}=$ EYEDEA & $\mathrm{M}=$ NEC \\
\hline $\mathrm{B}=$ HBINNO & $\mathrm{F}=$ VAPPLICA & $\mathrm{J}=$ HISIGN & $\mathrm{N}=$ TOSHIBA \\
\hline $\mathrm{C}=$ VIGILANT & $\mathrm{G}=$ MORPHO & $\mathrm{K}=$ COGNITEC & $\mathrm{Q}=$ IMAGUS \\
\hline $\mathrm{D}=$ AYONIX & $\mathrm{H}=3 \mathrm{M}$ COGENT & $\mathrm{L}=$ CYBEREXTRUDER & $\mathrm{R}=$ RANK ONE \\
\hline
\end{tabular}

\begin{tabular}{|l|l|l|l|l|l|}
\hline SET & SCENE & CAMERA & SET & SCENE & CAMERA \\
\hline C & PHOTOJOURNALISM & PRO & T & CONCOURSE & PRO \\
\hline J & PASSENGER LOADING & PRO & H & CONCOURSE & PRO \\
\hline P & SPORTS ARENA & CONSUMER & U & CHOKEPOINT & WEBCAM \\
\hline L & LUGGAGE RACK & WEBCAM & & & \\
\hline
\end{tabular}


and on the implementation cost.

See Table 20 and Figures $34-35$.

Effect of stalling a queue: When a ceiling mounted camera is used to identify subjects traversing a loading bridge, identification of subjects walking is much less successful than if an artificial bottleneck is used to cause the subjects to wait in a queue. The inset table shows miss rates for $N=480$ when a modest threshold is used to limit false positives to

\begin{tabular}{|l|r|r|r|r|}
\hline Accuracy & M & I & J & H \\
\hline Freely walking & $17 \%$ & $46 \%$ & $54 \%$ & $53 \%$ \\
\hline Queue stalled & $9 \%$ & $27 \%$ & $36 \%$ & $23 \%$ \\
\hline
\end{tabular}
ten during the 248 person experiment (without an attractor). These accuracy gains are available but with the cost that processing durations that are up to three times longer with queued subjects.

See Figure 17 and Table 33.

Effect of using an attractor: As face recognition algorithms have well documented sensitivity to head pose elevation, identification error rates increase with both the angle of the head relative to the optical axis of the camera, and with the difference of that angle to whatever it is in the enrollment photograph. It is common to install some device that attracts subjects' attention, and thereby improve pose. The audio-video attractor

\begin{tabular}{|l|r|r|r|r|}
\hline Attractor & M & I & N & H \\
\hline Off & $26 \%$ & $46 \%$ & $56 \%$ & $53 \%$ \\
\hline Digital mirror & $20 \%$ & $40 \%$ & $54 \%$ & $45 \%$ \\
\hline Agent audio video & $10 \%$ & $26 \%$ & $33 \%$ & $20 \%$ \\
\hline
\end{tabular}
reduced identification misses substantially. An alternative attractor, a live display of the subjects walking was less effective as it induced adverse behaviors (hand waving, exaggerated expressions). Subjects walked freely. The threshold is set to give 10 false positives in each condition.

See Figure 17.

Effect of multiple co-located cameras: Most results in the report relate to the failure to recognize an individual appearing before a single camera. However, when subjects walk freely through a volume that is concurrently imaged by three cameras, identification error rates vary between the cameras, and can be improved by fusion of scores produced during search. This was achieved by NIST implementing a max-score fusion across the three cameras. This step should more appropriately be done by passing multiple video streams to the algorithms, but this was not supported by the FIVE API. The threshold is set to give 10 false positives from each camera, and in the case of fusion, 10 from all cameras. The attractor was off.

\begin{tabular}{|l|r|r|r|r|}
\hline Position & $\mathrm{M}$ & $\mathrm{I}$ & $\mathrm{N}$ & $\mathrm{H}$ \\
\hline Ceiling & $26 \%$ & $46 \%$ & $63 \%$ & $53 \%$ \\
\hline Right & $35 \%$ & $64 \%$ & $70 \%$ & $62 \%$ \\
\hline Left & $26 \%$ & $50 \%$ & $60 \%$ & $53 \%$ \\
\hline Fusion & $16 \%$ & $38 \%$ & $58 \%$ & $41 \%$ \\
\hline
\end{tabular}

Capability to identify over multiple cameras and multiple locations: The prior paragraph addressed fusion of recognition hypotheses from multiple cameras observing a subject simultaneously. We ask two similar questions here. First, what proportion of subjects are missed if we fuse over all appearances before a single camera. This fusion over time (in the case of Dataset $\mathrm{P}$, a few hours), is effective if subjects are ever recognized. Second, we ask what proportion of subjects are missed if we fuse over all appearances before all cameras. This is fusion over space and time. The inset table shows fusion is very effective, suggesting installation of more cameras will improve accurary. Some obvious caveats, however: First, fusion over time will be ineffective if subjects don't appear on more than one occasion. Second, fusion over space will be ineffective if subjects only appear in one location. Third, fusion over time changes

\begin{tabular}{|l|l|r|r|r|r|}
\hline Fusion & Cameras & M & I & H & G \\
\hline None & low-near & $14 \%$ & $32 \%$ & $43 \%$ & $42 \%$ \\
\hline Over time & low near & $2 \%$ & $5 \%$ & $8 \%$ & $6 \%$ \\
\hline \hline None & All & $31 \%$ & $54 \%$ & $67 \%$ & $61 \%$ \\
\hline Over time and space & All & $1 \%$ & $4 \%$ & $6 \%$ & $1 \%$ \\
\hline
\end{tabular}
the operational relevance in that it is largely useless to know that a kleptomaniac has entered a shop only at the end of a day. The fused error rates are germane only to retroactive applications, like asylum claim backtracking in an airport, or non-repudiation.

See section 5.5.1.

Applicability in photojournalism: Imagery captured and published by professional photographs and television camera operators is distinguished by good illumination, focus, and composition. However it remains challenging because of diverse head poses and facial expressions. In a video-tostill mode, the inset table shows rank-1 miss rates are much higher than a ceiling-mounted surveillance setting, despite use of a gallery five times smaller. The reasons for this is

\begin{tabular}{|l|l|l|r|r|r|r|r|}
\hline Set & Use & Mode & Gallery & G & M & I & N \\
\hline C & Photojournalism & V2S & $\mathrm{N}=935$ & $36 \%$ & $42 \%$ & $50 \%$ & $52 \%$ \\
\hline H & Surveillance & V2S & $\mathrm{N}=4800$ & $28 \%$ & $16 \%$ & $17 \%$ & $19 \%$ \\
\hline H & Surveillance & V2S & $\mathrm{N}=48000$ & $48 \%$ & $26 \%$ & $50 \%$ & $37 \%$ \\
\hline
\end{tabular}
wider variation in pose in the photojournalism data, and that both the reference photograph and the search video are

\begin{tabular}{|l|l|l|l|}
\hline \multicolumn{5}{|c|}{ PARTICIPANT KEY } \\
\hline $\mathrm{A}=$ DIGITAL BARRIERS & $\mathrm{E}=$ NEUROTECHNOLOGY & $\mathrm{I}=$ EYEDEA & $\mathrm{M}=$ NEC \\
\hline $\mathrm{B}=$ HBINNO & $\mathrm{F}=$ VAPPLICA & $\mathrm{J}=$ HISIGN & $\mathrm{N}=$ TOSHIBA \\
\hline $\mathrm{C}=$ VIGILANT & $\mathrm{G}=$ MORPHO & $\mathrm{K}=$ COGNITEC & $\mathrm{Q}=$ IMAGUS \\
\hline $\mathrm{D}=$ AYONIX & $\mathrm{H}=$ 3M COGENT & $\mathrm{L}=$ CYBEREXTRUDER & $\mathrm{R}=$ RANK ONE \\
\hline
\end{tabular}

\begin{tabular}{|l|l|l|l|l|l|}
\hline SET & SCENE & CAMERA & SET & SCENE & CAMERA \\
\hline C & PHOTOJOURNALISM & PRO & T & CONCOURSE & PRO \\
\hline J & PASSENGER LOADING & PRO & H & CONCOURSE & PRO \\
\hline P & SPORTS ARENA & CONSUMER & U & CHOKEPOINT & WEBCAM \\
\hline L & LUGGAGE RACK & WEBCAM & & & \\
\hline
\end{tabular}


unconstrained. For the surveillance dataset $(\mathrm{H})$, the approximately frontal videos are searched against pristine frontal photographs.

See Table 31.

When photojournalism imagery is used in a video-to-video search application, identification miss rates are improved, as shown in the inset table. Here the task is to detect and enroll faces from 393 multi-person videos. This results in galleries containing from 1000 to 3000 tem-

\begin{tabular}{|l|l|l|r|r|r|r|r|r|}
\hline Set & Use & Mode & Gallery & G & M & I & N & H \\
\hline C & Photojournalism & V2S & N = 935 & $36 \%$ & $42 \%$ & $50 \%$ & $52 \%$ & $60 \%$ \\
\hline C & Photojournalism & V2V & N = Variable & $21 \%$ & $6 \%$ & $22 \%$ & $25 \%$ & $27 \%$ \\
\hline
\end{tabular}
plates, varying by algorithm. Then 1356 new videos each featuring one of the 393 main actors are then searched against the gallery. Accuracy is improved because any enrollment video will have more pose variation and a better likelihood to have some head pose in common with the search imagery.

See Figure 39.

Recently enormous development has been done using images of celebrities and politicians and more general amateur photos due to their internet availability. The important result here is that such imagery is more challenging than with video acquired from cameras whose installation is favorable. It suggests that cross-pose recognition of the human head (which often cannot be controlled) remains the primary impediment to better accuracy.

Computational resources for enrollment of images: For enrollment of single faces in still photographs, algorithms vary in their their execution duration and in the size of the template they produce. Template size can be important because it will drive memory requirements, and speed of communication (e.g. across a computer bus). It will be more important for embedded processors, and low power devices, and less important on server-class hardware. Template sizes range over three orders of magnitude, from 128 to 109150 bytes. The most accurate algorithm has size 2585 bytes. This range is indicative that the industry is far from consensus on the mathematical encoding of features from faces. Using a single server-class core, template generation durations range from 0.06 seconds to 1.49 seconds.

See Table 34 .

Computational resources for processing of videos: Recognition in video is computationally much more expensive than in still photographs. The duration of the entire video processing function scales with (at least) the image dimensions, the length of the video, and the number of faces. If an implementation extracted features from video frames using the same still-image technology - they don't - none of these algorithms would be capable of extracting features from 24 frames per second video in real-time on a single core. Instead, the implementations process entire video clips using dedicated algorithms implemented behind a FIVE API function call. This function is passed $k \gg 1$ frames (i.e. not single frames) thereby devolving responsibility of video processing to the algorithm. Internally the algorithm finds zero or more faces, tracks them through space and time, and extracts features, producing a template for each track. An algorithm could extract features from a best-frame, or it might integrate features over time. As a black box test, the particular techniques remain proprietary trade secrets. Only a small minority of the algorithms have speed sufficient to execute face recognition in video in real-time using a single server-class processing core. While the duration depends on how crowded a scene is, many of the more accurate algorithms can sustain processing of a single video stream in realtime on a single multi-core server. Deployers will need to weigh network and hardware costs, given expected search volumes. In addition, the offline nature of the FIVE test methodology does not simply translate to a mode of operation in which video is continuously streamed to a recognition engine, so software (and hardware) architectural effort will be necessary.

See Table 33

Privacy implications: The availability of effective means to biometrically identify persons in public spaces without their knowledge has been discussed in everything from Hollywood movies and dystopian novels to policy documents, government-led forums [4], public procurements, reports on regulation [11,29], and national strategy drafts [6]. In the the United States, legal issues arise primarily from the Fourth Amendment to the Constitition which prohibits "unreasonable searches and seizures" without "probable cause, supported by oath or affirmation" i.e. a warrant or "consent" which retires the requirement for a warrant. The constitutionality of acquiring faces (or other biometric data) in a public space without a warrant in order to identify or track an individual has not been considered: "The Supreme Court has

\begin{tabular}{|l|l|l|l|}
\hline \multicolumn{5}{|c|}{ PARTICIPANT KEY } \\
\hline $\mathrm{A}=$ DIGITAL BARRIERS & $\mathrm{E}=$ NEUROTECHNOLOGY & $\mathrm{I}=$ EYEDEA & $\mathrm{M}=$ NEC \\
\hline $\mathrm{B}=$ HBINNO & $\mathrm{F}=$ VAPPLICA & $\mathrm{J}=$ HISIGN & $\mathrm{N}=$ TOSHIBA \\
\hline $\mathrm{C}=$ VIGILANT & $\mathrm{G}=$ MORPHO & $\mathrm{K}=$ COGNITEC & $\mathrm{Q}=$ IMAGUS \\
\hline $\mathrm{D}=$ AYONIX & $\mathrm{H}=3 \mathrm{M}$ COGENT & $\mathrm{L}=$ CYBEREXTRUDER & $\mathrm{R}=$ RANK ONE \\
\hline
\end{tabular}

\begin{tabular}{|l|l|l|l|l|l|}
\hline SET & SCENE & CAMERA & SET & SCENE & CAMERA \\
\hline C & PHOTOJOURNALISM & PRO & T & CONCOURSE & PRO \\
\hline J & PASSENGER LOADING & PRO & H & CONCOURSE & PRO \\
\hline P & SPORTS ARENA & CONSUMER & U & CHOKEPOINT & WEBCAM \\
\hline L & LUGGAGE RACK & WEBCAM & & & \\
\hline
\end{tabular}


considered the Fourth Amendment implications of the police use of tracking beepers, electronic eavesdropping devices, photographic cameras with zoom lenses, and thermal-imaging devices, but not the use of video surveillance systems [9]. At issue will be whether any consent to be photographed additionally implies consent to storage (recording) of the result, enrollment by a feature extraction algorithm, and automated search. Would such consent be tied to a specific purpose, and would it last in perpetuity?

FIVE is germane to these issues because imaging resolution and duration have been deemed relevant in the courts. In 2016, in U.S. v. Houston [7], a warrantless search was permissible because "the use of the pole camera did not violate Houston's reasonable expectations of privacy because the camera recorded the same view of the farm as that enjoyed by passersby". In 2014, a lower court had reached the opposite conclusion in a very similar case, U.S. v. Vargas [5], which reflects why the opinion in Dow Chemical v. United States [1] noted "Fourth Amendment cases must be decided on the facts of each case, not by extravagant generalizations". In that case, the government prevailed because the aerial surveillance (of a Dow facility) was of low enough resolution that "No objects as small as 1/2-inch in diameter such as a class ring, for example, are recognizable, nor are there any identifiable human faces or secret documents captured in such a fashion as to implicate more serious privacy concerns." The implication, albeit in 1986, that face capture would trigger the Fourth Amendment, leads us to report image resolution needed for face recognition. Among the datasets evaluated here, the best accuracy is obtained from faces appearing in turnstile video clips with mean minimum and maximum interocular distances of 20 and 55 pixels respectively (See Table 9). These distances, which are lower than the 120 pixels mandated by the ISO / IEC 19794-5 passport specification, can readily be achieved with modern sensors and cameras placed tens of meters from the subject ${ }^{3}$, given adequate light. For resolution, the argument is "magnification may be functionally equivalent to a physical search of persons, papers, or effects" [9] in the same way that training specialized optics on a home can be tantamount to an unconstitutional search viz. Kyllo v. United States [2] which held that use of a "device that is not in general public use, to explore details of a private home that would previously have been unknowable without physical intrusion, the surveillance is a Fourth Amendment 'search,' and is presumptively unreasonable without a warrant".

Regarding duration (of surveillance), algorithms are capable of identifying faces that appear for very short durations below one second, and at frame rates well below that of broadcast video - and this means that a public figure might be identified at the entrance to a restaurant, or a protestor could be identified at a point on a march. This presumes the existence of a database of prior reference imagery, typically an authoritative database including portrait photographs and biographical data, but could also be any curated collection of photographs. This requires that subjects approximately face the camera (conservatively, to within 15 degrees) for a short interval (conservatively, for one second). See Figures 22 and 19 .

Human involvement and human fallibility: False positive and negative error rates are high enough that all consequential deployments of non-cooperative face recognition will require human adjudication of candidate matches. Prospective deployers must integrate human labor requirements into the workflow. In negative blacklist applications, where hits are expected to be rare, the system should be configured to yield (false) positives at a tractable rate. In positive whitelist applications, where all users are expected to yield a system response, and human review is not the default, the system must be configured to yield few enough false positives to satisfy security goals. Given that human review is implied and assumed in the operation of non-cooperative face recognition systems, and that humans commit recognition errors [14,33], readers should consider how the various errors from the human and automated parts of the hybrid system interact and how they can be mitigated. Note humans may be able to exploit non-face cues in video to video comparison [35].

Standards: There are standardization gaps associated with the use of automated face recognition. These are listed below. The first two are related to the necessity of human review of candidates from automated identification systems.

${ }^{3}$ For example, it is possible to sample 80 pixels across an interocular distance of $8 \mathrm{~cm}$ on a subject standing at 60 meters from a modern 36 megapixel SLR camera by using a 300mm lens set with an aperture of $\mathrm{f} 8$, achieving a depth of field of 3.4 meters. This configuration is likely expensive c. $\$ 10000$.

\begin{tabular}{|l|l|l|l|}
\hline \multicolumn{5}{|c|}{ PARTICIPANT KEY } \\
\hline $\mathrm{A}=$ DIGITAL BARRIERS & $\mathrm{E}=$ NEUROTECHNOLOGY & $\mathrm{I}=$ EYEDEA & $\mathrm{M}=$ NEC \\
\hline $\mathrm{B}=$ HBINNO & $\mathrm{F}=$ VAPPLICA & $\mathrm{J}=$ HISIGN & $\mathrm{N}=$ TOSHIBA \\
\hline $\mathrm{C}=$ VIGILANT & $\mathrm{G}=$ MORPHO & $\mathrm{K}=$ COGNITEC & $\mathrm{Q}=$ IMAGUS \\
\hline $\mathrm{D}=$ AYONIX & $\mathrm{H}=3 \mathrm{M}$ COGENT & $\mathrm{L}=$ CYBEREXTRUDER & $\mathrm{R}=$ RANK ONE \\
\hline
\end{tabular}

\begin{tabular}{|l|l|l|l|l|l|}
\hline SET & SCENE & CAMERA & SET & SCENE & CAMERA \\
\hline C & PHOTOJOURNALISM & PRO & T & CONCOURSE & PRO \\
\hline J & PASSENGER LOADING & PRO & H & CONCOURSE & PRO \\
\hline P & SPORTS ARENA & CONSUMER & U & CHOKEPOINT & WEBCAM \\
\hline L & LUGGAGE RACK & WEBCAM & & & \\
\hline
\end{tabular}


The remaining items support deployment of robust face recognition infrastructure.

$\diamond$ While a vibrant industry has developed around the frontal image types of the the ISO/IEC 19794-5 standard, this has had two unintended consequences. One is that because the standard recommends only 300 kilopixel images (640x480 "Token" geometry photographs), high resolution (multi-megapixel) images that are collected and used in their preparation are often not retained to support downstream review purposes. The standardized practice should be to collect high resolution, retain it for human review, and prepare the Token imagery from it for ingest into automated face recognition systems. Secondly, while the collection of frontal and profile views has existed in law enforcement since at least $1900^{4}$, it is not universally done, and collection of non-frontal views has been deprecated in most civil applications. The standardization gap is to formalize which non-frontal views best support human adjudication of potential false positives in one-to-many recognition.

$\diamond$ There is no standard for the display of photographs to human reviewers. The standard should specify: the optical display properties of the display device (resolution, color depth, gamma correction etc.); rotation and interpolation (magnification) algorithms; cropping; graphical user interface functionality, including with an integrated face recognition engine; procedures for the display of single photographs (e.g. for markup), and comparison of two hypothesized mated photographs. The standard should prohibit certain steps too, for example, certain image manipulation techniques such as alterations to parts of the face.

$\diamond$ Testing of biometric performance of video-based systems, e.g. surveillance systems, is non-trivial. Measurement techniques used in the FIVE study have been contributed toward development of a new standard: ISO/IEC 30137 Use of biometrics in video surveillance systems - Part 2: Performance testing and reporting. In early 2017, the standard is under development at the working draft stage.

$\diamond$ Installation of cameras for passive collection of faces for automated face recognition is a non-trivial process, particularly regarding geometry and optics. The FIVE results will be provided as quantitative support to the developement of the ISO/IEC 30137 - Use of biometrics in video surveillance systems - Part 1: Design and specification, now under development.

Conclusion: The accuracy of face recognition algorithms applied to the identification of non-cooperating individuals can approach that for the case of recognition of cooperating individuals in still photographs. This, however, will only be achieved if it is possible to repeatably capture similarly high quality frontal photographs. This is unlikely to occur as some proportion of a non-cooperating, passively imaged population will inevitably not present a suitable face image to the camera - for example, that population who happen to be wearing peaked hats while looking at a mobile phone. Others will present non-frontal faces. Further, high accuracy can only be achieved by deliberate installation and configuration of cameras and the environment, and such control over the deployment may sometimes be impossible, for physical, economic or societal reasons.

On the basis of the results in this report, the largest drivers of recognition accuracy are, in decreasing order of influence: algorithm selection; camera placement; the tolerable false positive rate for the particular application; subject walking speed and duration; gallery composition (portraits vs. selfies, for example); enrolled population size; camera type and the number of co-located cameras. This report provides some of the complex narratives around these variables, including interdependency.

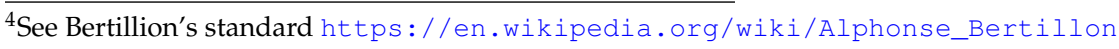

\begin{tabular}{|l|l|l|l|}
\hline \multicolumn{4}{|c|}{ PARTICIPANT KEY } \\
\hline A = DIGITAL BARRIERS & E = NEUROTECHNOLOGY & I = EYEDEA & M = NEC \\
\hline B = HBINNO & F = VAPPLICA & J = HISIGN & N = TOSHIBA \\
\hline C = VIGILANT & G = MORPHO & K = COGNITEC & Q = IMAGUS \\
\hline D = AYONIX & H = 3M COGENT & L = CYBEREXTRUDER & R = RANK ONE \\
\hline
\end{tabular}

\begin{tabular}{|l|l|l|l|l|l|}
\hline SET & SCENE & CAMERA & SET & SCENE & CAMERA \\
\hline C & PHOTOJOURNALISM & PRO & T & CONCOURSE & PRO \\
\hline J & PASSENGER LOADING & PRO & H & CONCOURSE & PRO \\
\hline P & SPORTS ARENA & CONSUMER & U & CHOKEPOINT & WEBCAM \\
\hline L & LUGGAGE RACK & WEBCAM & & & \\
\hline
\end{tabular}




\section{CAVEATS}

Readers are cauttioned that, as documentation of a laboratory test of core technology, this FIVE report is not a guide to the procurement of a face recognition enabled video based system, nor is it a complete mechanism to predict performance. The following specific cautions supplement those in the main text.

$\diamond$ Streaming: The FIVE study was conducted by passing single video clips to algorithms which output proprietary recognition templates and spatial face track information. This is done offline, in a batch process. This differs from online processing in which an algorithm receives a continuous video stream, and is asked to either detect and track faces in real-time or, further, to render identity hypotheses or decisions. Our approach is adopted to allow evaluation on large datasets and to achieve repeatability, but has two effects: First FIVE does not force algorithms to operate within some defined computation budget. Stated another way, it allows the algorithms to consume as many computer cycles as the developer deems necessary. To expose this, we measure and report computational expense. Secondly, it avoids the need to more use more complex accuracy measuring techniques. An online evaluation that allows algorithms to render real-time decisions from partial video sequences would measure the tradeoff between false negative and false positive identification rates and recognition time. The issues and proper conduct of such an evaluation has has been succintly described in the e-Passport gate context [16].

$\diamond$ Recent Development: The algorithms whose results appear in this report were submitted to NIST in Decemmber 2015. This report therefore does not document possible performance gains realized since that time. This is a consequence of mismatched evaluation vs. development schedules. It is not ideal, yet inevitable, that a report that attempts to broadly document the landscape of non-cooperative face recognition performance will take time longer than the development timescales of the core face recognition algorithms. This means that some suppliers will have progressed capability beyond that available in December 2015 when these algorithms were provided to the FIVE evaluation. This has motivated NIST to launch, in March 2017, an ongoing automated face recognition benchmark ${ }^{5}$.

$\diamond$ Same day recognition: Many of the trials described in the FIVE report include recognition of video collected on the same day as the enrollment still photographs. This is generally deprecated in performance testing standards such as ISO / IEC 19795 because it is known that accuracy is better for most biometric modalities when same-day matching is involved. This aspect is necessary for the cost-efficient collection of video data. We suggest that the increase in error rates from using say 30-day old photographs is small compared to the overall error rates, and the uncertainty in error rates from other sources. These include: population variance; population age, race and sex sample bias ${ }^{6}$; difficulty in reproducing cameras, compression procedures, environments, and algorithm parameterizations; and actor behavior departing from that expected operationally.

$\diamond$ Accuracy is not the entire story: Implementers should consider the following when considering which algorithms to integrate: cost; software maturity; software documentation; ease of programming; extensibility across servers and databases; performance requirements; accuracy reported here; accuracy reported in other independent test reports; accuracy dependence on image properties, such as resolution; dependence of accuracy on enrolled population size; template generation duration; search duration, and its dependence on enrolled population size.

\footnotetext{
${ }^{5}$ See https://www.nist.gov/programs-projects/face-recognition-vendor-test-frvt-ongoing.

${ }^{6}$ For example, younger people are harder to recognize
}

\begin{tabular}{|c|c|c|c|}
\hline \multicolumn{4}{|c|}{ PARTICIPANT KEY } \\
\hline $\mathrm{A}=$ DIGITAL BARRIERS & $\mathrm{E}=\mathrm{NEUROTECHNOLOGY}$ & $I=$ EYEDEA & $M=N E C$ \\
\hline $\mathrm{B}=\mathrm{HBINNO}$ & $\mathrm{F}=$ VAPPLICA & $\mathrm{J}=\mathrm{HISIGN}$ & $\mathrm{N}=$ TOSHIBA \\
\hline $\mathrm{C}=$ VIGILANT & $G=$ MORPHO & $\mathrm{K}=$ COGNITEC & $\mathrm{Q}=$ IMAGUS \\
\hline $\mathrm{D}=\mathrm{AYONIX}$ & $\mathrm{H}=3 \mathrm{M}$ COGENT & $\mathrm{L}=$ CYBEREXTRUDER & $\mathrm{R}=\mathrm{RANK}$ ONE \\
\hline
\end{tabular}

\begin{tabular}{|l|l|l|l|l|l|}
\hline SET & SCENE & CAMERA & SET & SCENE & CAMERA \\
\hline C & PHOTOJOURNALISM & PRO & T & CONCOURSE & PRO \\
\hline J & PASSENGER LOADING & PRO & H & CONCOURSE & PRO \\
\hline P & SPORTS ARENA & CONSUMER & U & CHOKEPOINT & WEBCAM \\
\hline L & LUGGAGE RACK & WEBCAM & & & \\
\hline
\end{tabular}




\section{RELEASE NOTES}

FIVE Reports: The results of the FIVE appear as a series of NIST Interagency Reports. All reports are linked from https://www.nist.gov/programs-projects/face-video-evaluation-five and its sub-pages.

Typesetting: Virtually all of the tabulated content in this report was produced automatically. This involved the use of scripting tools to generate directly type-settable $\mathrm{LT}_{\mathrm{E}} \mathrm{X}$ content. This improves timeliness, flexibility, maintainability, and reduces transcription errors.

Graphics: Many of the figures in this report were produced using Hadley Wickham's ggplot2 package running under $\mathbb{R}$, the capabilities of which extend beyond those evident in this document.

Contact: Correspondence regarding this report should be directed to FIVE at NIST dot GOV.

\begin{tabular}{|l|l|l|l|}
\hline \multicolumn{4}{|c|}{ PARTICIPANT KEY } \\
\hline A = DIGITAL BARRIERS & E = NEUROTECHNOLOGY & I = EYEDEA & $\mathrm{M}=$ NEC \\
\hline B = HBINNO & F = VAPPLICA & J = HISIGN & N = TOSHIBA \\
\hline C = VIGILANT & G = MORPHO & K = COGNITEC & Q = IMAGUS \\
\hline D = AYONIX & H = 3M COGENT & L = CYBEREXTRUDER & R = RANK ONE \\
\hline
\end{tabular}

\begin{tabular}{|l|l|l|l|l|l|}
\hline SET & SCENE & CAMERA & SET & SCENE & CAMERA \\
\hline C & PHOTOJOURNALISM & PRO & T & CONCOURSE & PRO \\
\hline J & PASSENGER LOADING & PRO & H & CONCOURSE & PRO \\
\hline P & SPORTS ARENA & CONSUMER & U & CHOKEPOINT & WEBCAM \\
\hline L & LUGGAGE RACK & WEBCAM & & & \\
\hline
\end{tabular}




\section{Contents}

ACKNOWLEDGEMENTS

DisClAimER

EXECUTIVE SUMMARY

BACKGROUND

TECHNICAL SUMMARY 8

CAVEATS

RELEASE Notes

1 INTRODUCTION 20

2 PARTicipation 20

3 TEST DESIgN 21

4 ACCURACY METRICS 21

4.1 Limits ON GROUND TRUTH ANNOTATION ACCURACY . . . . . . . . . . . . . . . . . . . . . . . . . 22

4.2 QUANTIFYING FALSE NEGATIVE ACCURACY . . . . . . . . . . . . . . . . . . . . . . . 23

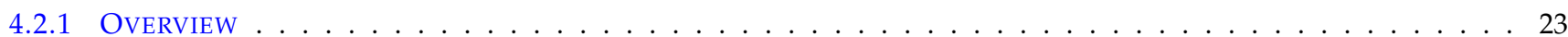

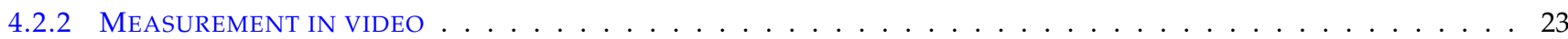

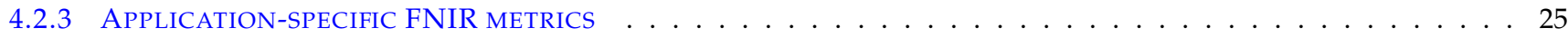

4.3 QUANTIFYING FALSE POSITIVE ACCURACY . . . . . . . . . . . . . . . . . . . . . . . 26

4.4 UNCERTAINTY IMPLIED BY INCOMPLETE GROUND TRUTH . . . . . . . . . . . . . . . . . . . . . . . 27

5 EXPERIMENTS AND RESULTS

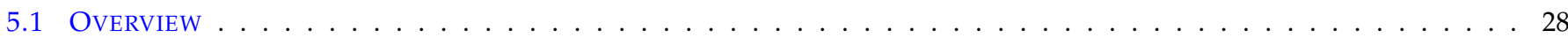

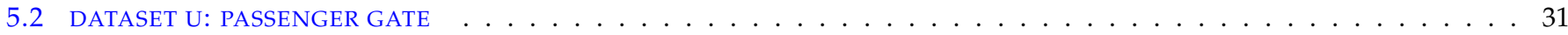

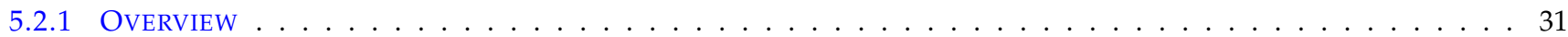

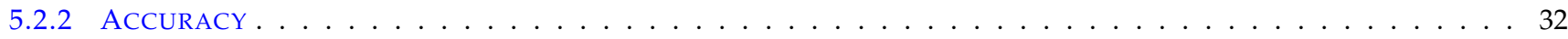

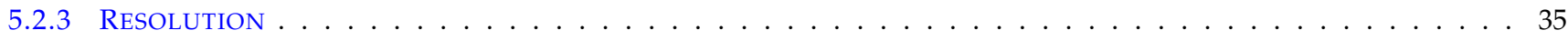

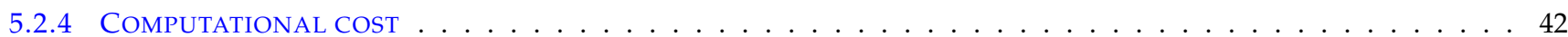

5.3 DATASET J: PASSENGER LOADING BRIDGE . . . . . . . . . . . . . . . . . . . . . . . . 46

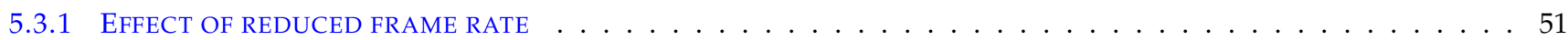

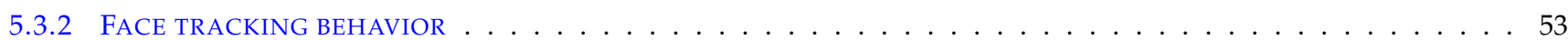

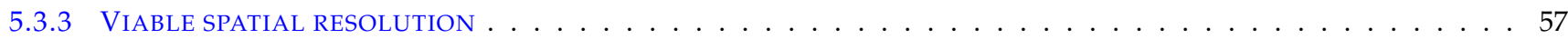

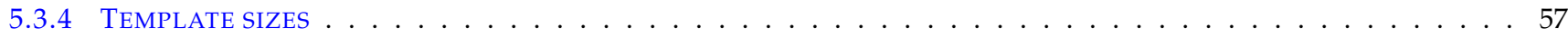

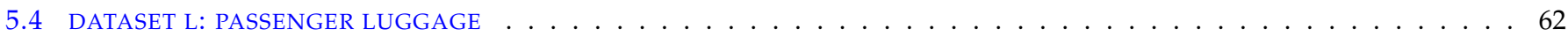

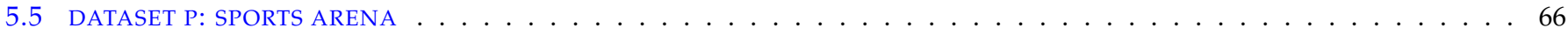

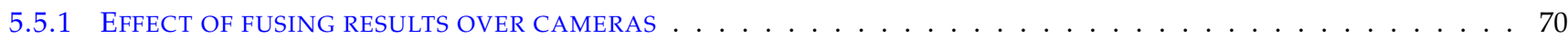

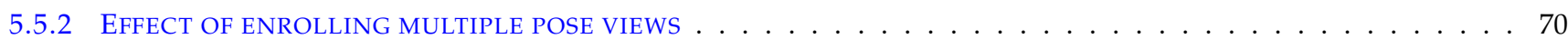

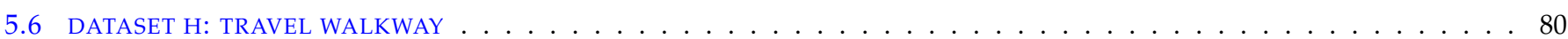

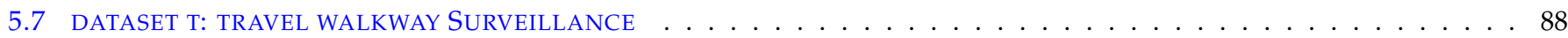

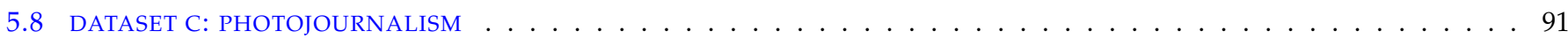

6 COMPUTATIONAL RESOURCE REQUIREMENTS 95

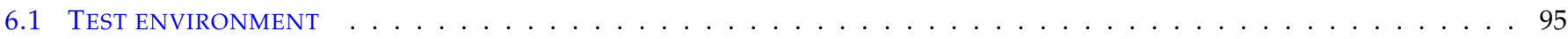

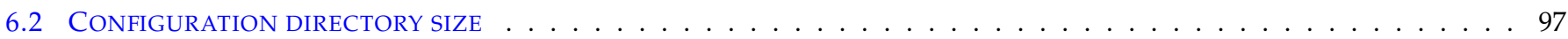

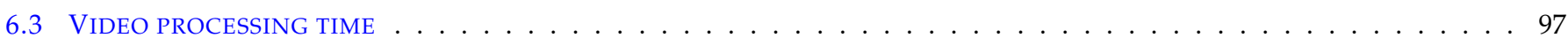

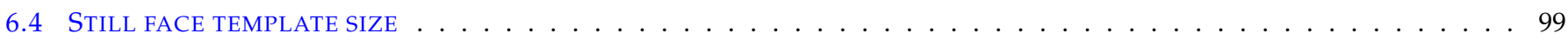

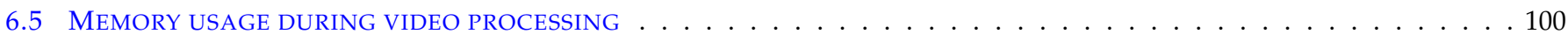

TABLES OF INTEROCULAR DISTANCES 105

\begin{tabular}{|c|c|c|c|c|c|c|c|c|c|}
\hline \multicolumn{4}{|c|}{ PARTICIPANT KEY } & \multirow{2}{*}{\begin{tabular}{|l|} 
SET \\
$\mathrm{C}$ \\
\end{tabular}} & SCENE & \multirow{2}{*}{\begin{tabular}{|l|} 
CAMERA \\
PRO
\end{tabular}} & \multirow{2}{*}{\begin{tabular}{|l|} 
SET \\
$\mathrm{T}$ \\
\end{tabular}} & \multirow{2}{*}{\begin{tabular}{|l|} 
SCENE \\
CONCOURSE \\
\end{tabular}} & \multirow{2}{*}{\begin{tabular}{|l} 
CAMERA \\
PRO
\end{tabular}} \\
\hline $\mathrm{A}=$ DIGITAL BARRIERS & $\mathrm{E}=$ NEUROTECHNOLOGY & $\mathrm{I}$ = EYEDEA & $M=N E C$ & & PHOTOJOURNALISM & & & & \\
\hline $\mathrm{C}=$ VIGILANT & $\mathrm{G}=\mathrm{MORPHO}$ & $\mathrm{K}=$ COGNITEC & $Q=$ IMAGUS & $\mathrm{P}$ & SPORTS ARENA & CONSUMER & $\mathrm{U}$ & CHOKEPOINT & WEBCAM \\
\hline $\mathrm{D}=$ AYONIX & $\mathrm{H}=3 \mathrm{M}$ COGENT & $\mathrm{L}=$ CYBEREXTRUDER & $\mathrm{R}=\mathrm{RANK}$ ONE & $\mathrm{L}$ & LUGGAGE RACK & WEBCAM & & & \\
\hline
\end{tabular}




\section{List of Tables}

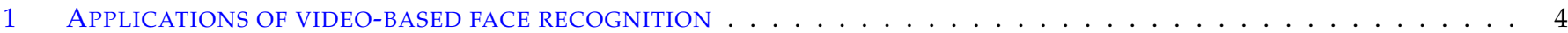

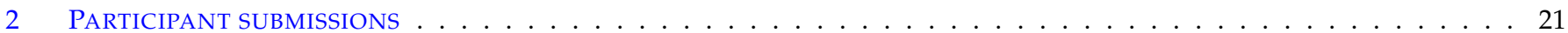

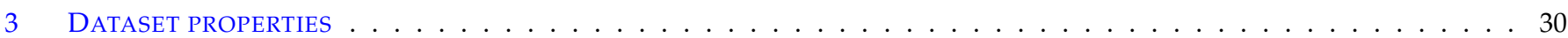

4 DATASET U: PASSENGER GATE KEY IMAGING PROPERTIES . . . . . . . . . . . . . . . . . . . . . . . . . 31

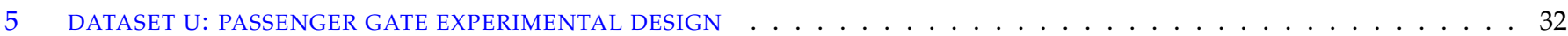

6 DATASET U: PASSENGER GATE ACCURACY SUMMARY, $\mathrm{N}=480 \ldots \ldots \ldots \ldots$

7 DATASET U: PASSENGER GATE ACCURACY SUMMARY, $\mathrm{N}=4800 \ldots \ldots \ldots \ldots$

8 DATASET U: PASSENGER GATE ACCURACY SUMMARY, $\mathrm{N}=48000 \ldots \ldots \ldots$

9 DATASET U: PASSENGER GATE TRACK AND INTEROCULAR DISTANCE STATISTICS . . . . . . . . . . . . . . . . 42

10 DATASET J: PASSENGER LOADING BRIDGE KEY IMAGING PROPERTIES . . . . . . . . . . . . . . . . . . . . . . . . 46

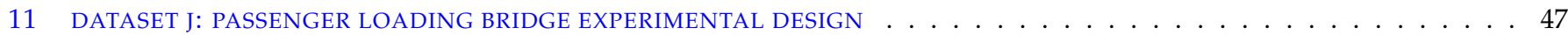

12 DATASET J: PASSENGER LOADING BRIDGE TRACK AND INTEROCULAR DISTANCE STATISTICS . . . . . . . . . . . . . 61

13 DATASET L: PASSENGER LUGGAGE KEY IMAGING PROPERTIES . . . . . . . . . . . . . . . . . . . . . 62

14 DATASET L: PASSENGER LUGGAGE EXPERIMENTAL DESIGN . . . . . . . . . . . . . . . . . . . . . 62

15 DATASET L: PASSENGER LUGGAGE TRACK AND INTEROCULAR DISTANCE STATISTICS . . . . . . . . . . . . . 64

16 DATASET L: PASSENGER LUGGAGE ACCURACY SUMMARY . . . . . . . . . . . . . . . . . . . . . . 65

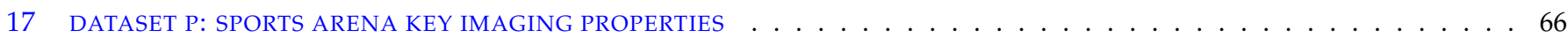

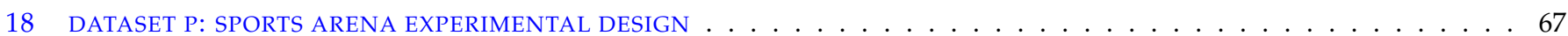

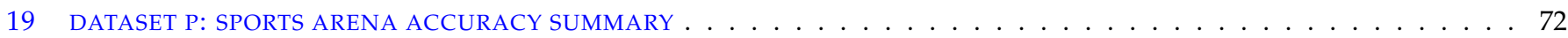

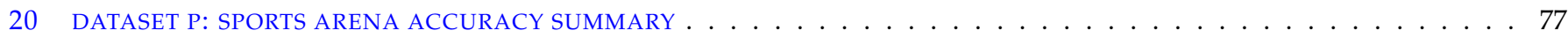

21 DATASET H: TRAVEL WALKWAY KEY IMAGING PROPERTIES . . . . . . . . . . . . . . . . . . . . . 80

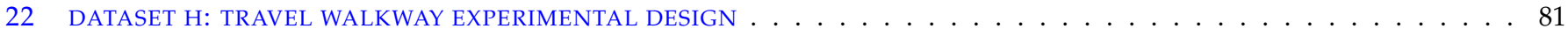

23 DATASET H: TRAVEL WALKWAY ACCURACY SUMMARY: CAMERAS ALL, $\mathrm{N}=480 \ldots \ldots$

24 DATASET H: TRAVEL WALKWAY ACCURACY SUMMARY: CAMERAS ALL, $\mathrm{N}=4800 \ldots \ldots$. . . . . . . . . . . 84

25 DATASET H: TRAVEL WALKWAY ACCURACY SUMMARY: CAMERAS ALL, $\mathrm{N}=48000 \ldots \ldots$. . . . . . . . . . . 85

26 DATASET H: TRAVEL WALKWAY ACCURACY SUMMARY COMPARING CAMERA SITES . . . . . . . . . . . . 86

27 DATASET T: TRAVEL WALKWAY ACCURACY SUMMARY $\ldots \ldots \ldots \ldots$

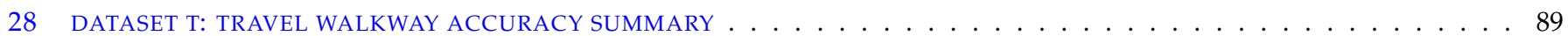

29 DATASET C: PHOTOJOURNALISM KEY IMAGING PROPERTIES . . . . . . . . . . . . . . . . . . . . . . . . 91

30 DATASET C: PHOTOJOURNALISM EXPERIMENTAL DESIGN . . . . . . . . . . . . . . . . . . . . . 93

31 DATASET C: PHOTOJOURNALISM ACCURACY SUMMARY . . . . . . . . . . . . . . . . . . . . . . . 94

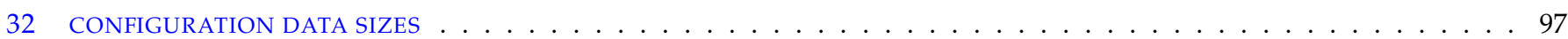

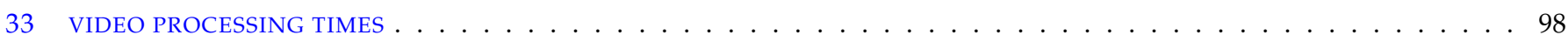

34 Template Size And Generation Time for Still Face IMAges . . . . . . . . . . . . . . . . . . . . . . 99

35 Template Size And Times for Still Face IMAGes . . . . . . . . . . . . . . . . . . . . . . . . 100

36 DATASET P: SPORTS ARENA TRACK AND INTEROCULAR DISTANCE STATISTICS . . . . . . . . . . . . . . . . 105

37 DATASET P: SPORTS ARENA TRACK AND INTEROCULAR DISTANCE STATISTICS . . . . . . . . . . . . . . . . 106

38 DATASET P: SPORTS ARENA TRACK AND INTEROCULAR DISTANCE STATISTICS . . . . . . . . . . . . . . . . . . . . . . . . . . . . .

39 DATASET P: SPORTS ARENA TRACK AND INTEROCULAR DISTANCE STATISTICS . . . . . . . . . . . . . . . . 108

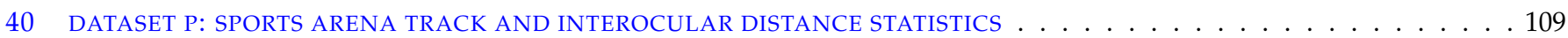

41 DATASET H: TRAVEL WALKWAY TRACK AND INTEROCULAR DISTANCE STATISTICS . . . . . . . . . . . . . . . . . 110

42 DATASET H: TRAVEL WALKWAY TRACK AND INTEROCULAR DISTANCE STATISTICS . . . . . . . . . . . . . . . . . 111

43 DATASET H: TRAVEL WALKWAY TRACK AND INTEROCULAR DISTANCE STATISTICS . . . . . . . . . . . . . . . 112

44 DATASET C: PHOTOJOURNALISM TRACK AND INTEROCULAR DISTANCE STATISTICS . . . . . . . . . . . . . . . 113

\section{List of Figures}

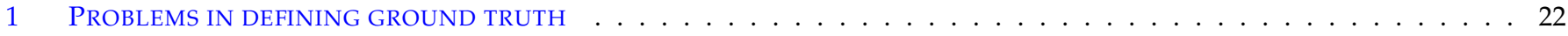

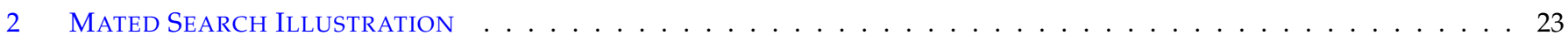

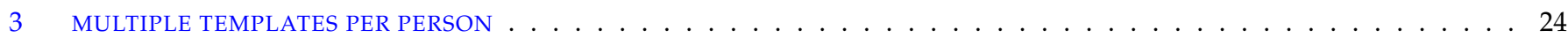

\begin{tabular}{|c|c|c|c|c|c|c|c|c|c|}
\hline \multicolumn{4}{|c|}{ PARTICIPANT KEY } & \multicolumn{2}{|c|}{\begin{tabular}{|l|l|} 
SET & SCENE \\
\end{tabular}} & \multirow{2}{*}{\begin{tabular}{|l|} 
CAMERA \\
PRO
\end{tabular}} & \multirow{2}{*}{\begin{tabular}{|l|} 
SET \\
$\mathrm{T}$ \\
\end{tabular}} & \multirow{2}{*}{\begin{tabular}{|l|} 
SCENE \\
CONCOURSE \\
\end{tabular}} & \multirow{2}{*}{\begin{tabular}{|l|} 
CAMERA \\
PRO
\end{tabular}} \\
\hline $\mathrm{A}=$ DIGITAL BARRIERS & $\mathrm{E}=\mathrm{NEUROTECHNOLOGY}$ & $\mathrm{I}=$ EYEDEA & $M=N E C$ & C & PHOTOJOURNALISM & & & & \\
\hline $\mathrm{C}=$ VIGILANT & $\mathrm{G}=\mathrm{MORPHO}$ & $\mathrm{K}=$ COGNITEC & $\mathrm{Q}=\mathrm{IMAGUS}$ & $\mathrm{P}$ & SPORTS ARENA & CONSUMER & $\mathrm{U}$ & CHOKEPOINT & WEBCAM \\
\hline $\mathrm{D}=$ AYONIX & $\mathrm{H}=3 \mathrm{M}$ COGENT & $\mathrm{L}=$ CYBEREXTRUDER & $\mathrm{R}=$ RANK ONE & $\mathrm{L}$ & LUGGAGE RACK & WEBCAM & & & \\
\hline
\end{tabular}




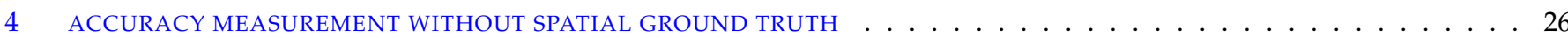

5 ACCURACY MEASUREMENT WITHOUT SPATIAL GROUND TRUTH $\ldots \ldots \ldots \ldots$

6 TEMPORAL ANNOTATION OF SUBJECT IDENTITY . . . . . . . . . . . . . . . . . . . . . . . . . . . . . 29

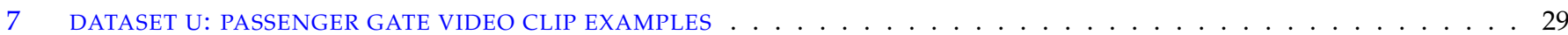

8 DATASET U: PASSENGER GATE ENROLLMENT IMAGE EXAMPLES . . . . . . . . . . . . . . . . . . . 31

9 DATASET U: PASSENGER GATE FNIR ACCURACY BAR PLOT . . . . . . . . . . . . . . . . . . . . . 36

10 DATASET U: PASSENGER GATE ERROR TRADEOFF CHARACTERISTICS . . . . . . . . . . . . . . . . . 40

11 DATASET U: PASSENGER GATE SCALABILITY TO LARGE POPULATIONS . . . . . . . . . . . . . . . . . . . . 41

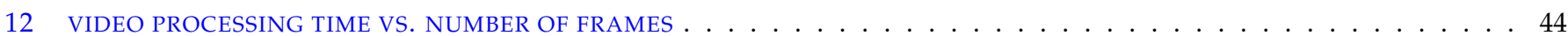

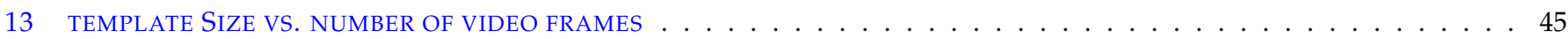

14 DATASET J: PASSENGER LOADING BRIDGE VIDEO CLIP EXAMPLES . . . . . . . . . . . . . . . . . . . . . 46

15 DATASET J: PASSENGER LOADING BRIDGE VIDEO CLIP EXAMPLES . . . . . . . . . . . . . . . . . . . . . 46

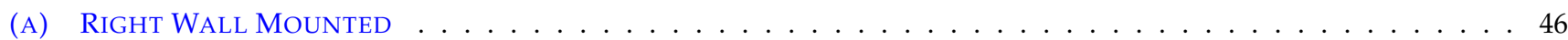

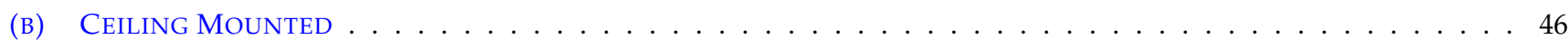

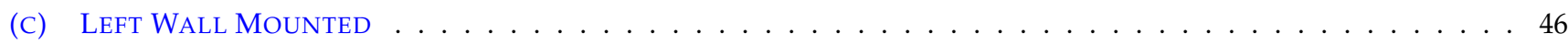

16 DATASET J: PASSENGER LOADING BRIDGE ENROLLMENT IMAGE EXAMPLES . . . . . . . . . . . . . . . . . . . 48

17 DATASET J: PASSENGER LOADING BRIDGE THRESHOLD-BASED ACCURACY . . . . . . . . . . . . . . . . . . . 49

18 DATASET J: PASSENGER LOADING BRIDGE RANK-BASED ACCURACY . . . . . . . . . . . . . . . . . . . 50

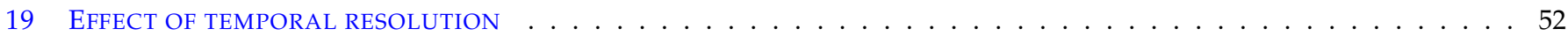

20 EXAMPLES Of IMAGES FROM THE DATASET J: PASSENGER LOADING BRIDGE DATASET . . . . . . . . . . . . . . . 53

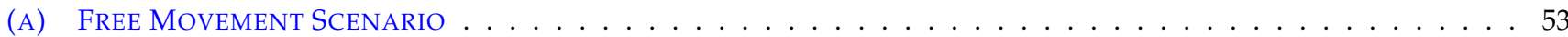

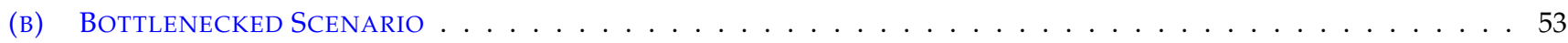

21 Detection COUNTS IN THE DATASET J: PASSENGER LOADING BRIDGE DATASET . . . . . . . . . . . . . . . . . . 55

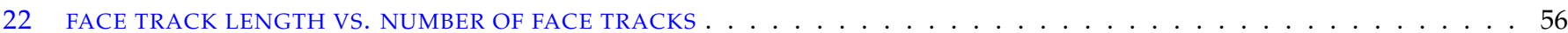

23 DATASET J: PASSENGER LOADING BRIDGE INTEROCULAR DISTANCE . . . . . . . . . . . . . . . . . . . . 58

24 DATASET J: PASSENGER LOAdING BRIDGE VIDEO TEMPLATE SIZES ． . . . . . . . . . . . . . . . . . . . . . 59

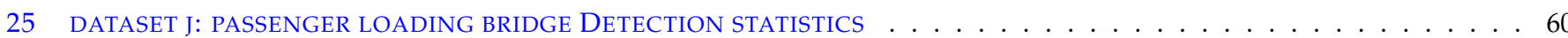

26 DATASET L: PASSENGER LUGgAgE VIDEO CLIP EXAMPLE . . . . . . . . . . . . . . . . . . . . . 63

28 DATASET P: SPORTS ARENA VIDEO CLIP EXAMPLES . . . . . . . . . . . . . . . . . . . . . . . . 67

27 DATASET P: SPORTS ARENA VIDEO CLIP EXAMPLES . . . . . . . . . . . . . . . . . . . . . . 68

29 DATASET P: SPORTS ARENA FNIR ACCURACY BAR PLOT . . . . . . . . . . . . . . . . . . . . . . . 71

30 DATASET P: SPORTS ARENA DETECTION AND FALSE POSITIVE COUNTS BY CAMERA LOCATION . . . . . . . . . . . . 73

31 DATASET P: SPORTS ARENA EFFECT OF FUSION OVER ALL SIGHTINGS . . . . . . . . . . . . . . . . . . . . . . 74

32 DATASET P: SPORTS ARENA EFFECT OF FUSION OVER ALL SIGHTINGS . . . . . . . . . . . . . . . . . . . . . . . . 75

33 DATASET P: SPORTS ARENA ENROLLMENT IMAGE EXAMPLES . . . . . . . . . . . . . . . . . 76

34 DATASET P: SPORTS ARENA EFFECT OF RICH GALLERY ON MATE RANK . . . . . . . . . . . . . . . . . . . 78

35 DATASET P: SPORTS ARENA EFFECT OF RICH GALLERY ON MATE SCORE . . . . . . . . . . . . . . . . . . . . . 79

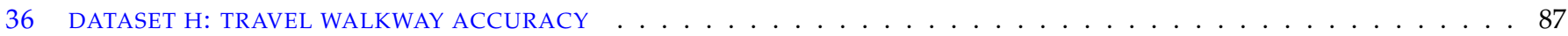

37 DATASET C: PHOTOJOURNALISM ENROLLMENT IMAGE EXAMPLES . . . . . . . . . . . . . . . . 91

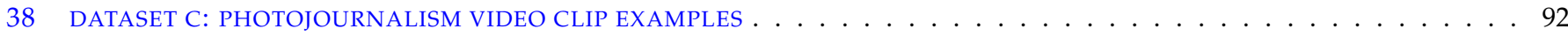

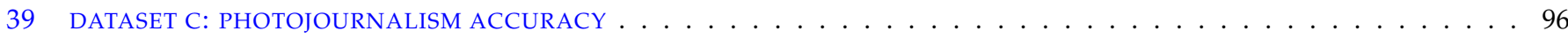

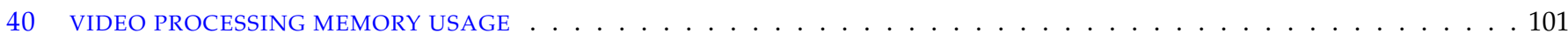

\begin{tabular}{|c|c|c|c|c|c|c|c|c|c|}
\hline \multicolumn{4}{|c|}{ PARTICIPANT KEY } & \multirow{2}{*}{\begin{tabular}{|l|} 
SET \\
$\mathrm{C}$ \\
\end{tabular}} & SCENE & \multirow{2}{*}{\begin{tabular}{|l|} 
CAMERA \\
PRO
\end{tabular}} & \multirow{2}{*}{\begin{tabular}{|l|} 
SET \\
$T$
\end{tabular}} & \multirow{2}{*}{\begin{tabular}{|l|} 
SCENE \\
CONCOURSE \\
\end{tabular}} & \multirow{2}{*}{\begin{tabular}{|l|} 
CAMERA \\
PRO
\end{tabular}} \\
\hline $\mathrm{A}=$ DIGITAL $\mathrm{B}$. & $E=$ NEUROTECHNOLOGY & $I=$ EYEDEA & $M=N E C$ & & PHOTOJOURNALISM & & & & \\
\hline $\mathrm{C}=$ VIGILANT & $G=\mathrm{MORPHO}$ & $\mathrm{K}=$ COGNITEC & $Q=$ IMAGUS & $P$ & SPORTS ARENA & CONSUMER & $\mathrm{U}$ & CHOKEPOINT & WEBCAM \\
\hline $\mathrm{D}=\mathrm{AYONIX}$ & $\mathrm{H}=3 \mathrm{M}$ COGENT & $\mathrm{L}=$ CYBEREXTRUDER & $\mathrm{R}=\mathrm{RANK}$ ONE & $\mathrm{L}$ & LUGGAGE RACK & WEBCAM & & & \\
\hline
\end{tabular}




\section{Introduction}

The Face In Video Evaluation (FIVE) was conducted to assess the capability of face recognition algorithms to correctly identify or ignore persons appearing in video sequences i.e. the open-set identification problem. This test was intended to support a plural marketplace of face recognition in video systems as there is considerable interest in the potential use of face recognition for identification of persons in videos.

NIST initiated FIVE in the second half of 2014. The evaluation was focused on one-to-many identification tests for video sequences. The test was intended to represent identification applications for face recognition in video, which included:

- Video-to-Still(V2S): This scenario supports identification of persons in video sequences against a gallery of enrolled stills, which has application in watch-list surveillance for example.

- Still-to-Video(S2V): This scenario supports identification of persons in stills against a gallery of identities enrolled from videos, which may have application in media search and asylum re-identification.

- Video-to-Video(V2V): This scenario supports identification of persons in video sequences against a gallery of identities enrolled from videos, which may have application in identity clustering and re-identification.

Out of scope: Areas that are out of scope for this evaluation and were not studied include: One-to-one verification of identity claims; identification from body worn cameras, license plate cameras, and aerial vehicles; video analytics, scene understanding, anomaly detection, and spatial boundary violation; suspicious behavior and intent detection; estimation of emotional state; gait recognition.

\section{Participation}

The FIVE program was open to participation worldwide. The participation window opened on November 17, 2014, and submission to the final phase closed on December 11,2015. There was no charge to participate.

The process and format of algorithm submissions to NIST was described in the FIVE Concept, Evaluation Plan and Application Programming Interface (API) document [20]. Participants provided their submissions in the form of libraries compiled on a specified Linux kernel, which were linked against NIST's test harness to produce executables. NIST provided a validation package to participants to ensure that NIST's execution of submitted libraries produced the expected output on NIST's test machines. FIVE had three submission phases where participants could submit algorithms to NIST. Results from phase 1 and 2 were provided back to the participants and are not documented in this report. This report documents the results of all algorithms submitted in the final phase (phase 3). Table 2 lists the FIVE participants, the letter code associated with the submitting organization, and the number of submissions made in each phase. The letter codes assigned to the participants are also located at the bottom of each page for reference.

Note that neither social media companies nor academic institutions elected to submit algorithms, and this report therefore only captures their capabilities to the extent that those technologies have been adopted or licensed by FIVE participants.

\begin{tabular}{|l|l|l|l|}
\hline \multicolumn{5}{|c|}{ PARTICIPANT KEY } \\
\hline $\mathrm{A}=$ DIGITAL BARRIERS & $\mathrm{E}=$ NEUROTECHNOLOGY & $\mathrm{I}=$ EYEDEA & $\mathrm{M}=$ NEC \\
\hline $\mathrm{B}=$ HBINNO & $\mathrm{F}=$ VAPPLICA & $\mathrm{J}=$ HISIGN & $\mathrm{N}=$ TOSHIBA \\
\hline $\mathrm{C}=$ VIGILANT & $\mathrm{G}=$ MORPHO & $\mathrm{K}=$ COGNITEC & $\mathrm{Q}=$ IMAGUS \\
\hline $\mathrm{D}=$ AYONIX & $\mathrm{H}=$ 3M COGENT & $\mathrm{L}=$ CYBEREXTRUDER & $\mathrm{R}=$ RANK ONE \\
\hline
\end{tabular}

\begin{tabular}{|l|l|l|l|l|l|}
\hline SET & SCENE & CAMERA & SET & SCENE & CAMERA \\
\hline C & PHOTOJOURNALISM & PRO & T & CONCOURSE & PRO \\
\hline J & PASSENGER LOADING & PRO & H & CONCOURSE & PRO \\
\hline P & SPORTS ARENA & CONSUMER & U & CHOKEPOINT & WEBCAM \\
\hline L & LUGGAGE RACK & WEBCAM & & & \\
\hline
\end{tabular}




\begin{tabular}{|l|l|c|c|c||c|}
\hline $\begin{array}{c}\text { Letter } \\
\text { Code }\end{array}$ & Organization & $\begin{array}{c}\text { Phase 1 } \\
\text { (February 2015) }\end{array}$ & $\begin{array}{c}\text { Phase 2 } \\
\text { (June 2015) }\end{array}$ & $\begin{array}{c}\text { Phase 3 } \\
\text { (December 2015) }\end{array}$ & Total \# Submissions \\
\hline A & Digital Barriers & 1 & 2 & 2 & 5 \\
\hline B & HBInno & 1 & 1 & 1 & 3 \\
\hline C & Vigilant Solutions & 1 & 1 & 2 & 4 \\
\hline D & Ayonix & 1 & 1 & 2 & 5 \\
\hline E & Neurotechnology & 1 & 2 & 2 & 2 \\
\hline F & Vapplica & 1 & & 1 & 5 \\
\hline G & Safran Morpho & 1 & 1 & 3 & 5 \\
\hline H & 3M Cogent & 1 & 1 & 3 & 5 \\
\hline I & Eyedea Recognition & 1 & 2 & 2 & 5 \\
\hline J & Beijing Hisign Technology & & 2 & 3 & 1 \\
\hline K & Cognitec Systems & & 1 & 1 & 5 \\
\hline L & CyberExtruder & & & 3 & 5 \\
\hline M & NEC Corporation & & 1 & 4 & 2 \\
\hline N & Toshiba Corporation & & & 2 & 1 \\
\hline Q & Imagus & & & & 5 \\
\hline R & Rank One Computing & & & \\
\hline
\end{tabular}

Table 2: FIVE participants, and the number of algorithm submissions, by phase.

\section{Test design}

Offline evaluations: The evaluation was conducted by applying algorithms to video and still imagery that is sequestered on computers controlled by NIST. Such offline tests are attractive because they allow uniform, fair, repeatable, and largescale statistically robust testing. Most of the imagery was collected in separate collection activities staged over the last few years, the one exception being the photojournalism imagery which was produced over many years, gathered from the internet, and assembled for use at NIST.

No algorithm bias: The collection activities were conducted without any recognition algorithm involvement i.e. there is no quality bias that would result if a face recognition algorithm had been involved in the selection or retention of any images.

Operational representativeness: The degree to which the results realized in this test can be replicated in operational settings depends on many factors, the foremost of which would be collection of imagery with the same properties as that used here. The germane properties are those that generically affect face recognition performance, i.e. orientation of the face relative to the optical axis (pose), optical resolution, illumination, video compression and frame rate, and human behavior.

This test is advantaged over many biometric evaluations in that most of the video imagery is collected in a single pass without explicit subject cooperation and system feedback. The absence of a live interactive component means that offline evaluation can readily repeat what would have happened if recognition had been attempted as soon as the imagery was streamed to a receiving system.

\section{Accuracy metrics}

This section describes how accuracy is measured and reported. Biometric algorithms commit three kinds of errors: failures to acquire ${ }^{7}$, and false negatives and false positives. In the context of this report, failures to acquire occur when

\footnotetext{
${ }^{7}$ The term failure to acquire is an overloaded phrase: It can mean failure of the face detection algorithm to find a face; it can mean the choice by the algorithm to not compute features from a face it deems to have poor utility for recognition; it might even refer to software failures.

\begin{tabular}{|l|l|l|l|}
\hline \multicolumn{4}{|c|}{ PARTICIPANT KEY } \\
\hline A = DIGITAL BARRIERS & E = NEUROTECHNOLOGY & I = EYEDEA & M = NEC \\
\hline B = HBINNO & F = VAPPLICA & J = HISIGN & N $=$ TOSHIBA \\
\hline C = VIGILANT & G = MORPHO & K = COGNITEC & Q IMAGUS \\
\hline D = AYONIX & H = 3M COGENT & L = CYBEREXTRUDER & R = RANK ONE \\
\hline
\end{tabular}

\begin{tabular}{|l|l|l|l|l|l|}
\hline SET & SCENE & CAMERA & SET & SCENE & CAMERA \\
\hline C & PHOTOJOURNALISM & PRO & T & CONCOURSE & PRO \\
\hline J & PASSENGER LOADING & PRO & H & CONCOURSE & PRO \\
\hline P & SPORTS ARENA & CONSUMER & U & CHOKEPOINT & WEBCAM \\
\hline L & LUGGAGE RACK & WEBCAM & & & \\
\hline
\end{tabular}
}


a face is not detected in an image or video, false negatives are outcomes where a search should yield a specific enrolled identity but does not, and false positives correspond to a search returning enrolled identities when it should not. Before defining these more formally, we note that it is erroneous yet common to state performance using a single number, a practice which is incomplete and very often misleading. As described below, correct coverage should note several aspects: the tradeoff between false positives and negatives, the enrolled population size, the prior probabilities of known and unknown persons, the degree and type of human involvement, and the dependence on image quality which can render results only poorly portable to a new installation.

\subsection{Limits on ground truth annotation accuracy}

Before discussing recognition accuracy metrics, we first introduce the notion that measurement of accuracy in video surveillance is limited by the appearance of multiple people in a scene, their motion in and out of the scene, and their non-cooperative incidental presence in front of the camera. This complicates the question of actually how many people are present, something that is needed for a hard estimate of false positive identification rate.

When images are collected in non-staged settings and public spaces, for example from surveillance cameras or from photojournalists, and in other "in the wild" settings, the number of persons is not normally known. This is true in extended video sequences and still photographs. More importantly, the number of visible faces is not known, not least because "visibility" is poorly defined. Any human reviewer could sum the number of people present in Figure 1 but if a face only appears in two frames with 8 pixels between the eyes, does that count as a ground truth face? Does a person wearing sunglasses, a baseball hat and looking down at a mobile phone such that only his

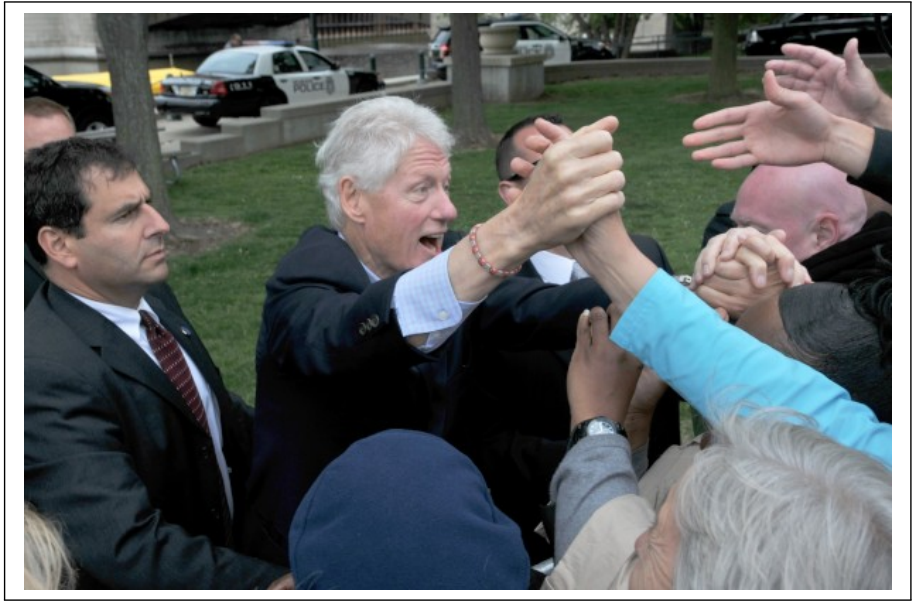

Figure 1: This image is an example of the difficulty in counting the number of faces available for recognition. This question comes in two parts: Given an enrollment image it is clear the main subject of the photo, President Bill Clinton, and the agent with the red tie could be included in a measurement of FNIR. While the bald man at right, and the police officer next to the car, could possibly be recognized by members of his family or inner circle, should an algorithm be tested on such images? The remaining faces are detectable as human beings but are arguably not recognizable. The second part is how many faces could be counted towards a measurement of FPIR? There are 13 individuals present in the photograph, but facial skin is only visible from seven. [Photo Credit: Marc Tasman. License: https://commons.wikimedia.org/wiki/ File:Bill_Clinton_Wisconsin.jpg] ${ }^{* *}$ This is image 913 in the IJBA dataset. It is distributed under a creative commons license.

chin is visible count as a recognizable face? In an operational utopia, both of these edge cases would be recognition opportunities, but in a practical sense, they are not - recognition will forever be uncertain. One rigorous, though not scalable, way forward is two establish two quantities. First is to count humans by providing imagery to an analyst with a play-forward play-backward reviewing capability and summing where the analyst can ascertain that a person was present, whether their face was at all visible or not. Second, is to count the number of recognizable faces by asking a skilled analyst to identify the faces in a very small gallery. This method is laborious, and subject to inter-rater variance. Given the volume of imagery, this procedure is not undertaken in FIVE.

Our conclusion here is that the ground truth number of faces present in non-cooperative video is often, not always, unknown and unknowable. The implication of this, as detailed below, is that the denominator in the false positive identification rate estimates is not known. Instead the operational metric is the count of false positives, rather than a rate. This

\begin{tabular}{|c|c|c|c|c|c|c|c|c|c|}
\hline \multicolumn{4}{|c|}{ PARTICIPANT KEY } & SET & SCENE & CAMERA & SET & SCENE & CAMERA \\
\hline $\mathrm{A}=$ DIGITAL BARRIERS & $\mathrm{E}=$ NEUROTECHNOLOGY & $\mathrm{I}=\mathrm{EYEDEA}$ & $M=N E C$ & C & PHOTOJOURNALISM & PRO & $\mathrm{T}$ & CONCOURSE & PRO \\
\hline $\mathrm{B}=\mathrm{HBINNO}$ & $\mathrm{F}=$ VAPPLICA & $\mathrm{J}=\mathrm{HISIGN}$ & $\mathrm{N}=$ TOSHIBA & $\mathrm{J}$ & PASSENGER LOADING & PRO & $\mathrm{H}$ & CONCOURSE & PRO \\
\hline$C=$ VIGILANT & $\mathrm{G}=\mathrm{MORPHO}$ & $\mathrm{K}=$ COGNITEC & $\mathrm{Q}=\mathrm{IMAGUS}$ & $\mathrm{P}$ & SPORTS ARENA & CONSUMER & $\mathrm{U}$ & CHOKEPOINT & WEBCAM \\
\hline $\mathrm{D}=$ AYONIX & $\mathrm{H}=3 \mathrm{M}$ COGENT & $\mathrm{L}=\mathrm{CYBEREXTRUDER}$ & $\mathrm{R}=\mathrm{RANK}$ ONE & $\mathrm{L}$ & LUGGAGE RACK & WEBCAM & & & \\
\hline
\end{tabular}




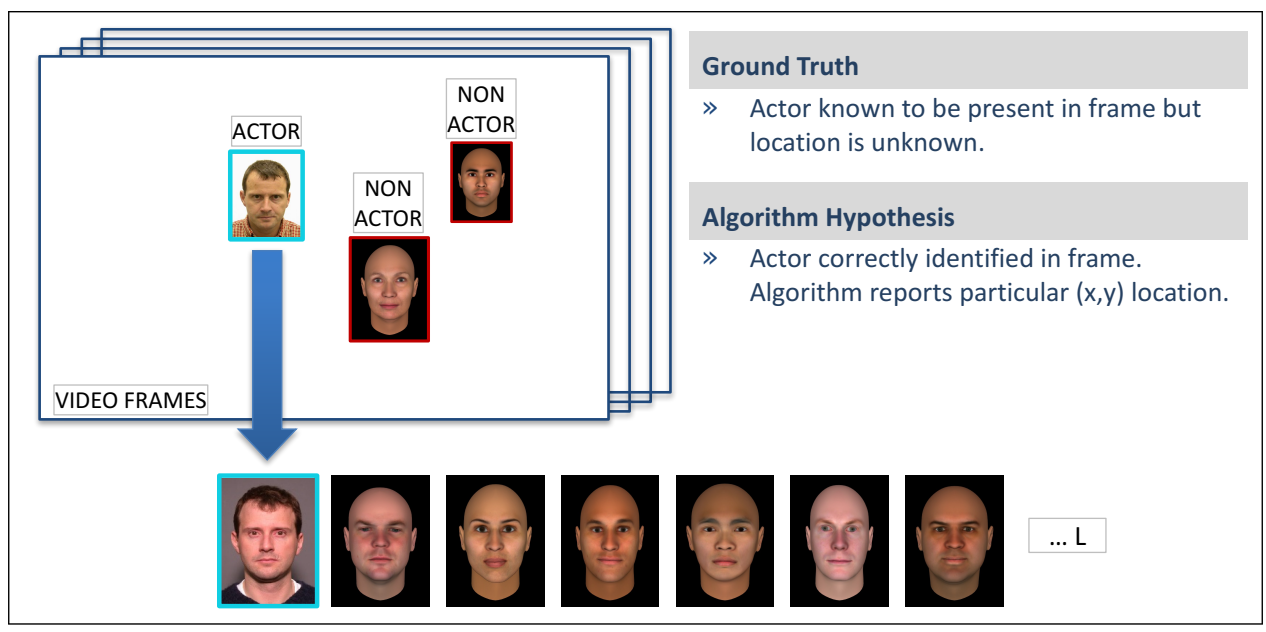

Figure 2: Illustration of a mated search. While three faces are present here, a video generally contains zero or more faces. The algorithm must detect and track faces across frames - it does not know a priori which, if any, faces are present in the enrolled gallery. The algorithm, which operates on the video sequence, produces a template for each track and this is searched against one or more enrollment galleries. The faces in this figure are of the first author or are synthetic.

does not have to be exact - an approximate estimate is sufficient as because other quality factors (resolution, pose) can alter false positive rates by larger amounts.

It could be argued that the number of known faces present is similarly unknown, such that the denominator in the false negative identification rate estimate is similarly unknown. However, in FIVE their presence is known, and their behaviour is expected to be that of the target population, they are all counted as recognition opportunities.

Annotation of faces in video imagery is now being standardized in ISO/IEC 30137 Use of biometrics in video surveillance systems - Part 4: Ground truth and video annotation procedure.

\subsection{Quantifying false negative accuracy}

\subsubsection{Overview}

The first error metric is the False Negative Identification Rate (FNIR) which can be regarded as a "miss rate". FNIR is the proportion of searches involving imagery of persons who are enrolled in the gallery which fail to produce the correct matching identity from the enrolled set of identities. FNIR is estimated by conducting mated searches (Figure 2) of people in videos or stills against an enrollment dataset where persons are known to be in both the search probe and the enrollment dataset.

\subsubsection{Measurement in video}

So far this definition is generic to biometrics; for faces in video clips we need more specificity. Particularly when the imagery is a video clip, two complications arise. First there can be many faces in the video. Second, a face detection and tracking algorithm may find one person on several occasions over the duration of the clip. Thus it may generate multiple templates for a single face track (see Figure 3), or incorrectly consolidate multiple face tracks into a single template. Such events must be appropriately reflected in the error metrics.

\begin{tabular}{|l|l|l|l|}
\hline \multicolumn{5}{|c|}{ PARTICIPANT KEY } \\
\hline $\mathrm{A}=$ DIGITAL BARRIERS & $\mathrm{E}=$ NEUROTECHNOLOGY & $\mathrm{I}=$ EYEDEA & $\mathrm{M}=$ NEC \\
\hline $\mathrm{B}=$ HBINNO & $\mathrm{F}=$ VAPPLICA & $\mathrm{J}=$ HISIGN & $\mathrm{N}=$ TOSHIBA \\
\hline $\mathrm{C}=$ VIGILANT & $\mathrm{G}=$ MORPHO & $\mathrm{K}=$ COGNITEC & $\mathrm{Q}=$ IMAGUS \\
\hline $\mathrm{D}=$ AYONIX & $\mathrm{H}=$ 3M COGENT & $\mathrm{L}=$ CYBEREXTRUDER & $\mathrm{R}=$ RANK ONE \\
\hline
\end{tabular}

\begin{tabular}{|l|l|l|l|l|l|}
\hline SET & SCENE & CAMERA & SET & SCENE & CAMERA \\
\hline C & PHOTOJOURNALISM & PRO & T & CONCOURSE & PRO \\
\hline J & PASSENGER LOADING & PRO & H & CONCOURSE & PRO \\
\hline P & SPORTS ARENA & CONSUMER & U & CHOKEPOINT & WEBCAM \\
\hline L & LUGGAGE RACK & WEBCAM & & & \\
\hline
\end{tabular}




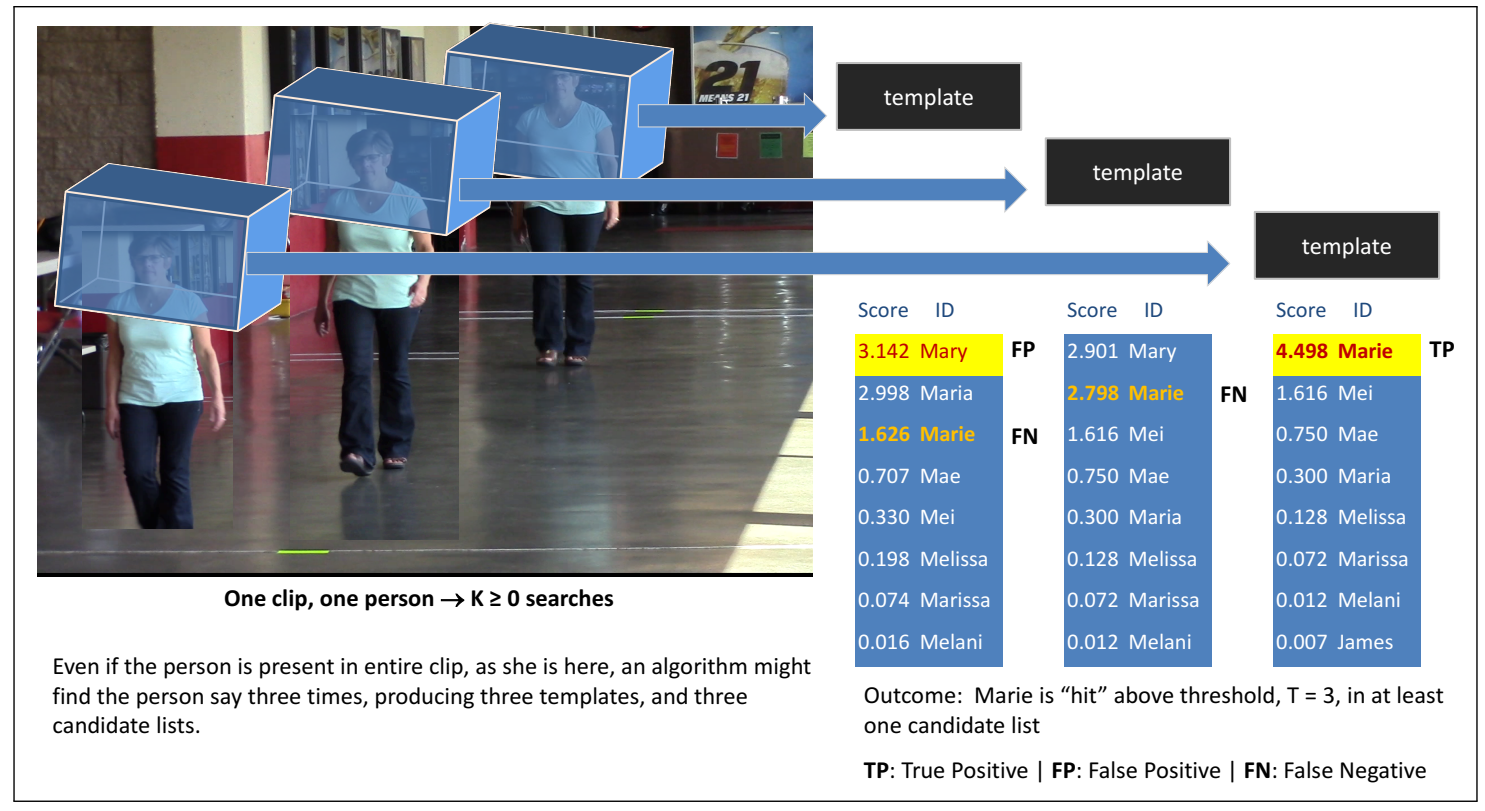

Figure 3: Example of how an algorithm may produce multiple templates for the same person/single face track in a video clip. ${ }^{* *}$ The face images in this figure are from a DHS S\&T provided dataset. We obtained written consent from DHS / S\&T to use these images in public reports.

Thus, given an input video, a recognition algorithm detects zero or more face tracks and produces a template from each. Each is searched against the enrolled database. The algorithm is required to return the $L$ closest (most similar) candidates. We quote accuracy by shortening the candidate list by either applying a threshold $T$, or by considering only the top $R \leq L$ candidates $^{8}$. Each candidate is comprised of a hypothesized identity, and a similarity score. The algorithm sorts the resulting candidate list in descending order of similarity score. Even when a clip contains only one person, we need to handle the detection and production of $K \geq 0$ candidate lists. Thus, for a video known to contain imagery of individual $i$ we take the maximum of $K$ mate scores or 0 if there are none.

Formally, when searching $\mathrm{N}$ identities, let $s_{i k r}$ be the comparison score associated with the $1 \leq r \leq L$-th ranked candidate from the $1 \leq k$-th candidate list from the $i$ th video, and let $p_{i k r}$ be the subject identifier for the $r$-th candidate from the same candidate list and video. Additionally, if $J_{i}$ is the number of templates returned for the $i$-th video clip, where $0<i \leq I$, and $M_{i}$ is the set of identifiers for subjects actually present in the $i$ th video then

$$
\operatorname{FNIR}(N, R, T)=\frac{\sum_{i=1}^{I} \sum_{j \in M_{i}} H\left[\max _{1 \leq r \leq R} \max _{1 \leq k \leq J_{i}}\left[s_{i k r} \mid p_{i k r}=j\right]-T\right]}{\sum_{i=1}^{I}\left|M_{i}\right|}
$$

where $\left|M_{i}\right|$ is the size of $M_{i}$ and the step function $H(x-T)$ is 1 if score $x$ is at or above threshold $T$. The denominator counts the total number of times subjects in the enrollment dataset appear in the video clips (only counting a subject

\footnotetext{
${ }^{8}$ To clarify the relationship between $\mathrm{L}$ and $\mathrm{R}: \mathrm{L}$ is the number of requested candidates that is communicated to the algorithm. Importantly setting $\mathrm{L}$ to a large value will generally cause search to go slower, so system administrators may not permit analysts to set $\mathrm{L}$ at all, or only to do with some bound. R, on the other hand, is the number of candidates that an analyst might look at in an application with a graphical user interface. The analyist is constrained to look at $R_{i}=L$. The performance metrics in this report survey over $R$ around, to see the effect on accuracy. Operationally, on a busy day, local workflow management software might limit analysts to look only at top $R=5$ canidates even though the underlying system was requested $\mathrm{L}=100$.
}

\begin{tabular}{|l|l|l|l|}
\hline \multicolumn{5}{|c|}{ PARTICIPANT KEY } \\
\hline $\mathrm{A}=$ DIGITAL BARRIERS & $\mathrm{E}=$ NEUROTECHNOLOGY & $\mathrm{I}=$ EYEDEA & $\mathrm{M}=$ NEC \\
\hline $\mathrm{B}=$ HBINNO & $\mathrm{F}=$ VAPPLICA & $\mathrm{J}=$ HISIGN & $\mathrm{N}=$ TOSHIBA \\
\hline $\mathrm{C}=$ VIGILANT & $\mathrm{G}=$ MORPHO & $\mathrm{K}=$ COGNITEC & $\mathrm{Q}=$ IMAGUS \\
\hline $\mathrm{D}=$ AYONIX & $\mathrm{H}=$ 3M COGENT & $\mathrm{L}=$ CYBEREXTRUDER & $\mathrm{R}=$ RANK ONE \\
\hline
\end{tabular}

\begin{tabular}{|l|l|l|l|l|l|}
\hline SET & SCENE & CAMERA & SET & SCENE & CAMERA \\
\hline C & PHOTOJOURNALISM & PRO & T & CONCOURSE & PRO \\
\hline J & PASSENGER LOADING & PRO & H & CONCOURSE & PRO \\
\hline P & SPORTS ARENA & CONSUMER & U & CHOKEPOINT & WEBCAM \\
\hline L & LUGGAGE RACK & WEBCAM & & & \\
\hline
\end{tabular}


multiple times if they appear in different video clips). Note that for each video clip, this equation only considers the highest score for an enrolled subject regardless of how many candidate lists the subject appears on. Thus for a given search clip, a correct identification occurs if the subject appears on at least one candidate list for the video clip with a score at or above threshold $T$ and rank no greater than $\mathrm{R}$. This gives no additional credit for correctly identifying a particular subject in a video clip several times. Algorithms vary considerably in how many templates they produce given the same input clip. This arises due to varying face detection methods and imperfect tracking of individuals across frames. Algorithms that generate more templates (and thus more candidate lists) have more opportunities to find the correct person. A "gaming strategy" might involve submitting an algorithm that produces very large numbers of templates essentially guessing at identities. Although this could reduce FNIR, it might also substantially elevate the number of false positives, particularly because some galleries here contain no actors (for measurement of FPIR - see section 4.3 below).

\subsubsection{Application-specific FNIR metrics}

The report includes extensive tabulation of FNIR reflecting two classes of use:

Forensic: In a high profile case, or in an application where only a few searches are ever conducted, a human analyst might examine say $1 \leq R \leq L$ candidates, where $\mathrm{L}$ is the maximum number available ${ }^{9}$ selecting $R$ according to the priority of the case, and labor availablity. The analyst might increase candidate list length, $L$, also to support a more laborious search for matching identities. In any case, the appropriate metric for this forensic use case, is a special case of equation 1, namely $\operatorname{FNIR}(N, R, 0)$ where threshold is set to zero so that all candidates can be available for review. This is a "miss rate" and is related to the ubiquitous cumulative match characteristics which states the proportion of searches with "hit" at rank R or better:

$$
\operatorname{CMC}(N, R)=1-F N I R(N, R, 0)
$$

By ignoring scores $(\mathrm{T}=0)$, this metric allows "weak" hits to count as strongly as high-scoring "strong" hits. Note the $\mathrm{CMC}$ metric is relevant to operations in which (trained) human reviewers who will traverse candidate lists in pursuit of hits are required and assumed. Their presence, in conjunction with a face recognition engine, forms a hybrid automatic-human system. The system functions only when when the volume of searches is low enough, and when the CMC is favorable enough, to occupy the available labor (and no more).

Surveillance: On the other hand, in applications such as surveillance in a public-space, where the prior probability of a mate is low, or where search volumes are very high and where human labor has limited availability, it becomes impossible to review all candidate lists. To limit workload a threshold $T$ is applied so that only candidates with score at or above threshold are provided for examination. The appropriate metric then is $\operatorname{FNIR}(N, L, T)$ where the rank criterion is relaxed by setting $\mathrm{R}$ equal to $\mathrm{L}$, and a non-zero threshold is applied ${ }^{10}$. High thresholds suppress

\footnotetext{
${ }^{9}$ Some biometric search implementations return only high scoring candidates. It is more common, however, for systems to return a specified number, $L$, candidates, and this value is communicated to the algorithm. This can be set by system policy, or sometimes by the analyst. In general, the duration of the search depends on L, the number of nearest neighbors are being sought, because multi-stage templates might be used, and because some fast search algorithms depend on the data. $R$ is the number of candidates that an analyst might look at in a GUI-enabled workstation. We distinguish the symbol $R$ from the length $L$ in order to that we may sweep it over its range $1 \leq R \leq L$ to see the effect on accuracy. Operationally, on a busy day, local workflow management software might limit analysts to look only at top $\mathrm{R}=5$ even though the underlying system was set to require $L=100$ from the algorithm.

${ }^{10}$ This formulation allows a mate to be at any rank $R<L$ as long as it is above threshold. Practically $\operatorname{FNIR}(N, L, T) \rightarrow$ FNIR $(N, 1, T)$ except when $T \rightarrow 0$. Once the threshold is elevated slightly sufficiently, mates are always found at rank 1 .
}

\begin{tabular}{|l|l|l|l|}
\hline \multicolumn{5}{|c|}{ PARTICIPANT KEY } \\
\hline $\mathrm{A}=$ DIGITAL BARRIERS & $\mathrm{E}=$ NEUROTECHNOLOGY & $\mathrm{I}=$ EYEDEA & $\mathrm{M}=$ NEC \\
\hline $\mathrm{B}=$ HBINNO & $\mathrm{F}=$ VAPPLICA & $\mathrm{J}=$ HISIGN & $\mathrm{N}=$ TOSHIBA \\
\hline $\mathrm{C}=$ VIGILANT & $\mathrm{G}=$ MORPHO & $\mathrm{K}=$ COGNITEC & $\mathrm{Q}=$ IMAGUS \\
\hline $\mathrm{D}=$ AYONIX & $\mathrm{H}=3 \mathrm{M}$ COGENT & $\mathrm{L}=$ CYBEREXTRUDER & $\mathrm{R}=$ RANK ONE \\
\hline
\end{tabular}

\begin{tabular}{|l|l|l|l|l|l|}
\hline SET & SCENE & CAMERA & SET & SCENE & CAMERA \\
\hline C & PHOTOJOURNALISM & PRO & T & CONCOURSE & PRO \\
\hline J & PASSENGER LOADING & PRO & H & CONCOURSE & PRO \\
\hline P & SPORTS ARENA & CONSUMER & U & CHOKEPOINT & WEBCAM \\
\hline L & LUGGAGE RACK & WEBCAM & & & \\
\hline
\end{tabular}




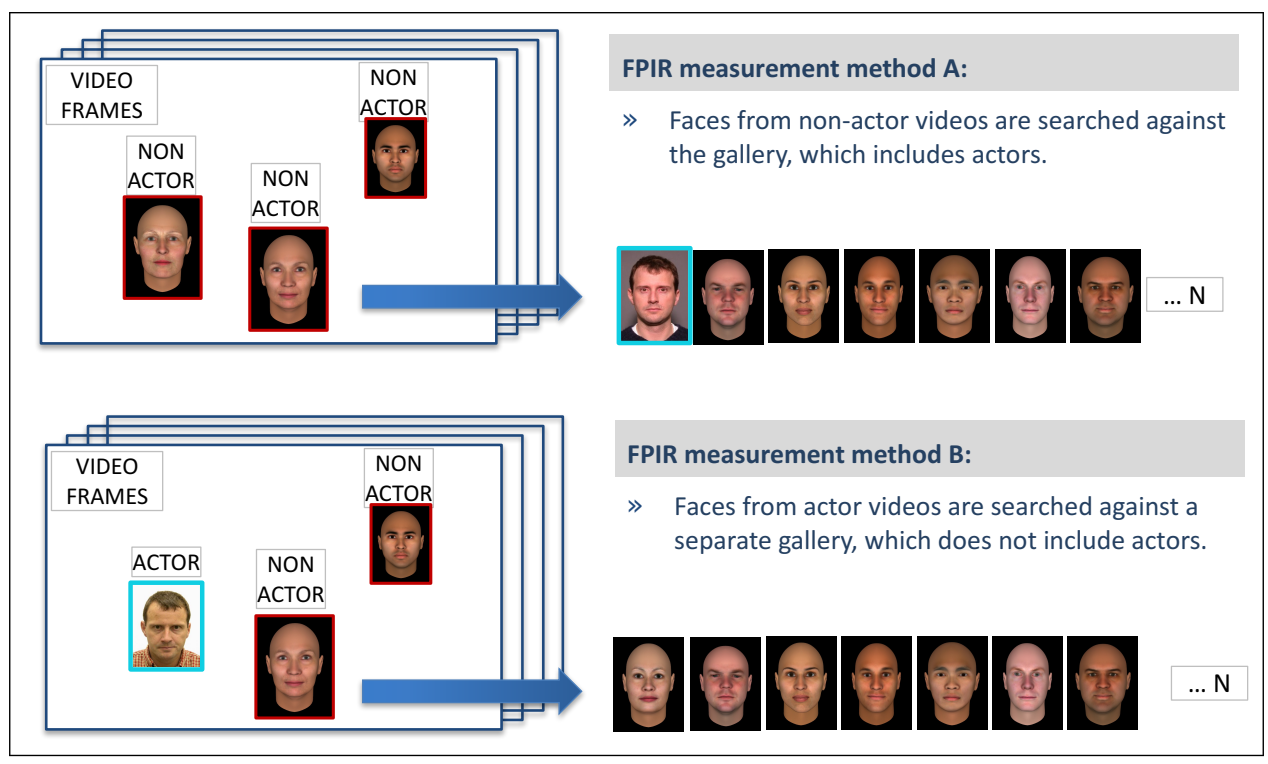

Figure 4: The figure shows two means of measuring FPIR. At top, videos containing faces not present in the gallery are used. Below the same set of videos used in measurement of FNIR are used but against a separate gallery not containing the actors. The faces in this figure are of the first author or are synthetic.

false positives, but elevate false negatives. For example, the 2007 German trial of a surveillance system in the Mainz train station [3] configured the threshold on each algorithms to target FPIR $=0.001$.

The threshold is set to limit the number of false positives. This is discussed in the next section.

\subsection{Quantifying false positive accuracy}

It is conventional in testing of biometric identification systems to measure the false positive identification rate (FPIR). This is done by running searches of individuals who are known to be absent from the enrolled gallery. FPIR is then computed as the proportion of searches that produces one or more false positives above a threshold, $\mathrm{T}$.

Here, this computation is not possible, because the number of individuals in the search imagery is not known, per the discussion of Figure 1. This leaves us to compute only a number of false positives from some searches. This still depends on the threshold, which we calibrate as follows. Given an input video, the recognition algorithm detects zero or more face tracks, producing a template from each. These are searched against a disjoint set of $\mathrm{N}$ individuals such that all reported candidates are, by definition, false positives. The threshold is set to the lowest value that results in a fixed number of observed false positives, denoted by $\operatorname{NFP}(T)$. This value is an integer rather than a proportion of the population.

As shown in Figure 4, NFP(T) is estimated in either of two ways: First by searching imagery of unrelated individuals against an enrolled actor gallery; and second by searching actor imagery against a gallery of unrelated indviduals. In a video-to-still experiment, the first of these methods necessitates collection of separate video, while the second method uses just the actor video but requires construction of a new, disjoint, gallery.

Most of the video clips used in this evaluation are fairly short ( $\leq 20$ or 30 seconds), and a particular subject only appears in a video clip once (although presentation of the face may be momentarily interrupted due to occlusion or pose changes).

To compute non-mated scores, in most cases the enrollment dataset was replaced with an equally sized set of frontal

\begin{tabular}{|l|l|l|l|}
\hline \multicolumn{5}{|c|}{ PARTICIPANT KEY } \\
\hline $\mathrm{A}=$ DIGITAL BARRIERS & $\mathrm{E}=$ NEUROTECHNOLOGY & $\mathrm{I}=$ EYEDEA & $\mathrm{M}=$ NEC \\
\hline $\mathrm{B}=$ HBINNO & $\mathrm{F}=$ VAPPLICA & $\mathrm{J}=$ HISIGN & $\mathrm{N}=$ TOSHIBA \\
\hline $\mathrm{C}=$ VIGILANT & $\mathrm{G}=$ MORPHO & $\mathrm{K}=$ COGNITEC & $\mathrm{Q}=$ IMAGUS \\
\hline $\mathrm{D}=$ AYONIX & $\mathrm{H}=$ 3M COGENT & $\mathrm{L}=$ CYBEREXTRUDER & $\mathrm{R}=$ RANK ONE \\
\hline
\end{tabular}

\begin{tabular}{|l|l|l|l|l|l|}
\hline SET & SCENE & CAMERA & SET & SCENE & CAMERA \\
\hline C & PHOTOJOURNALISM & PRO & T & CONCOURSE & PRO \\
\hline J & PASSENGER LOADING & PRO & H & CONCOURSE & PRO \\
\hline P & SPORTS ARENA & CONSUMER & U & CHOKEPOINT & WEBCAM \\
\hline L & LUGGAGE RACK & WEBCAM & & & \\
\hline
\end{tabular}




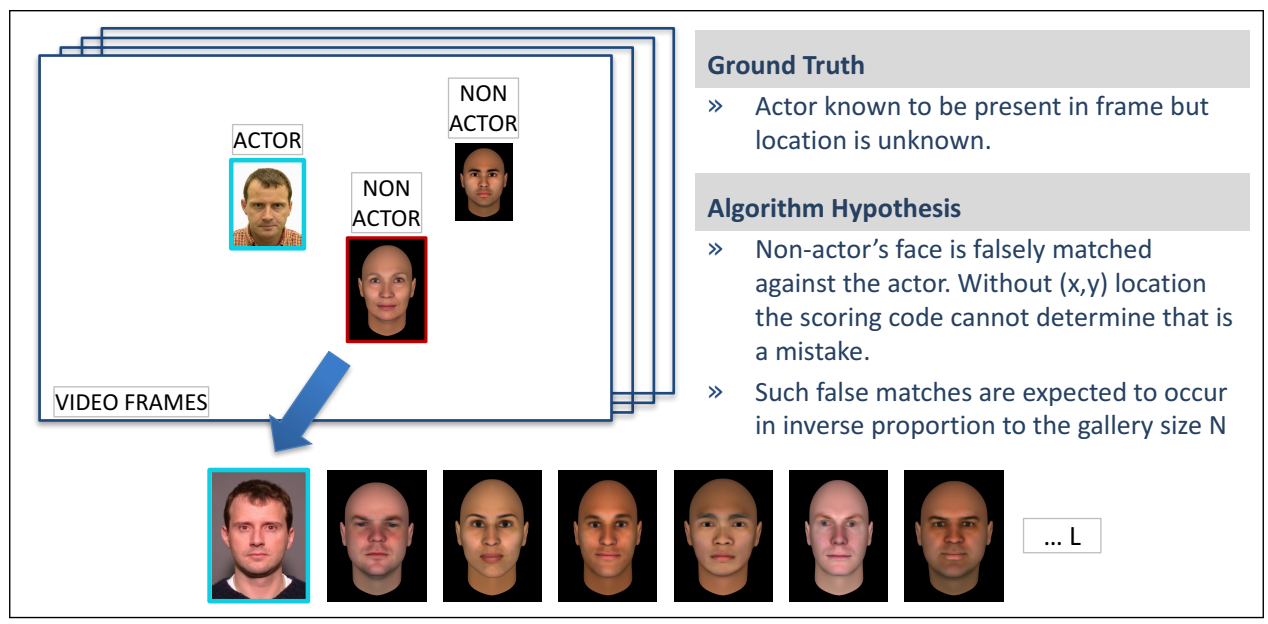

Figure 5: The figure shows that without location ground truth, it is possible for the algorithm to be credited with a correct identification of the actor, but for the wrong reason. The faces in this figure are of the first author or are synthetic.

stills of subjects that are not in any of the video clips. Let $q_{i j k}$ be the comparison score associated with the $k$ th candidate from the $j$ th candidate list from the $i$ th search. Since the enrollment dataset is populated with people not present in the video clips, all scores are non-mated. The number of false positives is computed as follows

$$
\operatorname{NFP}(T)=\sum_{i=1}^{I} \sum_{j=1}^{\left|J_{i}\right|} H\left(q_{i j 1}-T\right)
$$

NFP is the number of times a algorithm incorrectly flags someone as being in the enrollment dataset. Algorithms that detect more faces or produce more tracks will generate more false positives. The presence of the 1 subscript in $q$ indicates that a search produces a false positive if 1 or more (i.e. any) of the candidates are at or above threshold.

\subsection{Uncertainty implied by incomplete ground truth}

For a majority of the datasets, our evaluation methodology only checks that the correct person was found within a window, or in close proximity to the known ground truth. This is depicted in Figure 6. We do not test whether the subject was found in the correct location. This section describes the effect of this, and its mitigation.

Suppose we have search imagery where one actor and $n$ non-actors appear concurrently, and the actor has a mate in an $N$ person enrollment gallery. Suppose further we do not have spatiotemporal location information of the actor, i.e. we don't know where the actor is in the video. Suppose also that an algorithm detects all $n+1$ faces, tracks them, producing search templates and then candidate lists. There is some chance that a non-actor template incorrectly matches the actor's enrollment template randomly such that the accuracy computation counts a correct identifcation for the wrong reason see Figure 5. The probability that any one non-actor is returned as a match within the top $\mathrm{R}$ candidates can be obtained from the hypergeometric distribution as $R / N$. This applies on binomial grounds to all $n$ non-actors in the scene such that there is a systematic underestimation of the true FNIR as follows:

$$
\operatorname{FNIR}_{\mathrm{OBSERVED}}(N, R, 0)=\operatorname{FNIR}_{\mathrm{TRUE}}(N, R, 0)\left(1-\frac{R}{N}\right)^{n}
$$

\begin{tabular}{|l|l|l|l|}
\hline \multicolumn{4}{|c|}{ PARTICIPANT KEY } \\
\hline $\mathrm{A}=$ DIGITAL BARRIERS & $\mathrm{E}=$ NEUROTECHNOLOGY & $\mathrm{I}=$ EYEDEA & $\mathrm{M}=$ NEC \\
\hline $\mathrm{B}=$ HBINNO & $\mathrm{F}=$ VAPPLICA & $\mathrm{J}=$ HISIGN & $\mathrm{N}=$ TOSHIBA \\
\hline $\mathrm{C}=$ VIGILANT & $\mathrm{G}=$ MORPHO & $\mathrm{K}=$ COGNITEC & $\mathrm{Q}=$ IMAGUS \\
\hline $\mathrm{D}=$ AYONIX & $\mathrm{H}=$ 3M COGENT & $\mathrm{L}=$ CYBEREXTRUDER & $\mathrm{R}=$ RANK ONE \\
\hline
\end{tabular}

\begin{tabular}{|l|l|l|l|l|l|}
\hline SET & SCENE & CAMERA & SET & SCENE & CAMERA \\
\hline C & PHOTOJOURNALISM & PRO & T & CONCOURSE & PRO \\
\hline $\mathrm{J}$ & PASSENGER LOADING & PRO & H & CONCOURSE & PRO \\
\hline P & SPORTS ARENA & CONSUMER & U & CHOKEPOINT & WEBCAM \\
\hline L & LUGGAGE RACK & WEBCAM & & & \\
\hline
\end{tabular}


which has an approximation for small $\mathrm{R}^{11}$.

To gauge the worst case magnitude of this error, we examine the case of $\mathrm{N}=480$ (the smallest gallery we used), $\mathrm{R}=$ 20 (the highest rank we used), and $n=10$ (the approximate maximum number of people present in a clip). There the observed FNIR may understate the actual FNIR by a factor of 0.65 . This is large. For rank 1 recognition, the factor is 0.98 . For our largest gallery, $\mathrm{N}=48000$, the factor becomes negligible even at rank 20 (0.996).

However, thus far the analysis has been about random association of a non-actor with an actor. But face recognition algorithms do not place items on candidate lists randomly ${ }^{12}$, rather they nominate candidates in decreasing order of similarity score, rendering the hypergeometric model incorrect. This improves the situation markedly because the probability that a non-actor incorrectly matches the specific actor is related to the one-to-one false match rate, which reduces with threshold. This means that equation 4 is pessimistic, and particularly becomes irrelevant when the threshold is increased to limit false positives. A number of other aspects mitigate the problem further.

1. $\mathrm{N}$ is often much larger than our minimum here, $\mathrm{N}=480$.

2. For comparison of algorithms $\mathrm{R}=1$ is more appropriate.

3. We are mostly concerned with high threshold cases, particularly for the crowded surveillance datasets, $\mathrm{H}$ and $\mathrm{T}$.

4. For datasets $\mathrm{U}, \mathrm{J}, \mathrm{C}$, the number of people in the field of view, $n$, is often naturally near to 1 .

5. For datasets $\mathrm{J}, \mathrm{L}, \mathrm{H}$ and $\mathrm{T}$, the temporal ground truth is localized tightly such that the number of people present is usually below 5 . Thus even in video clips lasting several minutes (dataset J) where footage of hundreds of indviduals appeared, we imposed the additional constraint that the matching software must report the subject over the correct time interval (See Figure 6).

Nevertheless we add the caveat to some tables in this report, for $N=480$ and $R=20$, to direct readers to the arguments of this section.

This issue has been discussed previously [8].

\section{Experiments and results}

\subsection{Overview}

The prior section gave formal exposition of the metrics. This section previews the experiment runs that support the results that follow. Algorithm performance is assessed over seven biometric datasets. The datasets differ with respect to the types of cameras used for collection, their placement and number, the background environment, and many unknown and difficult to document factors. Each dataset roughly imitates a particular scenario. In all cases, user cooperation is essentially non-cooperative, meaning the people in the videos were variously unaware, not-cognizant, or oblivious to the presence of cameras. In one dataset, the camera was mounted alongside an attractor (computer display). It was included with the intent to induce head elevation and thereby a more frontal pose. In all cases, subjects were not given instructions

\footnotetext{
${ }^{11}$ With $1-(1-p)^{N} \rightarrow p N$ for small $p N$, the approximate formula is $\operatorname{FNIR}_{\operatorname{OBSERVED}}(N, R, 0)=\operatorname{FNIR}_{\mathrm{TRUE}}(N, R, 0)(1-n R / N)$

${ }^{12}$ Except in cases where the image quality is very poor.

\begin{tabular}{|l|l|l|l|}
\hline \multicolumn{4}{|c|}{ PARTICIPANT KEY } \\
\hline A = DIGITAL BARRIERS & E = NEUROTECHNOLOGY & I = EYEDEA & M = NEC \\
\hline B = HBINNO & F = VAPPLICA & J = HISIGN & N = TOSHIBA \\
\hline C = VIGILANT & G = MORPHO & K = COGNITEC & Q = IMAGUS \\
\hline D = AYONIX & H = 3M COGENT & L = CYBEREXTRUDER & R = RANK ONE \\
\hline
\end{tabular}

\begin{tabular}{|l|l|l|l|l|l|}
\hline SET & SCENE & CAMERA & SET & SCENE & CAMERA \\
\hline C & PHOTOJOURNALISM & PRO & T & CONCOURSE & PRO \\
\hline J & PASSENGER LOADING & PRO & H & CONCOURSE & PRO \\
\hline P & SPORTS ARENA & CONSUMER & U & CHOKEPOINT & WEBCAM \\
\hline L & LUGGAGE RACK & WEBCAM & & & \\
\hline
\end{tabular}
}




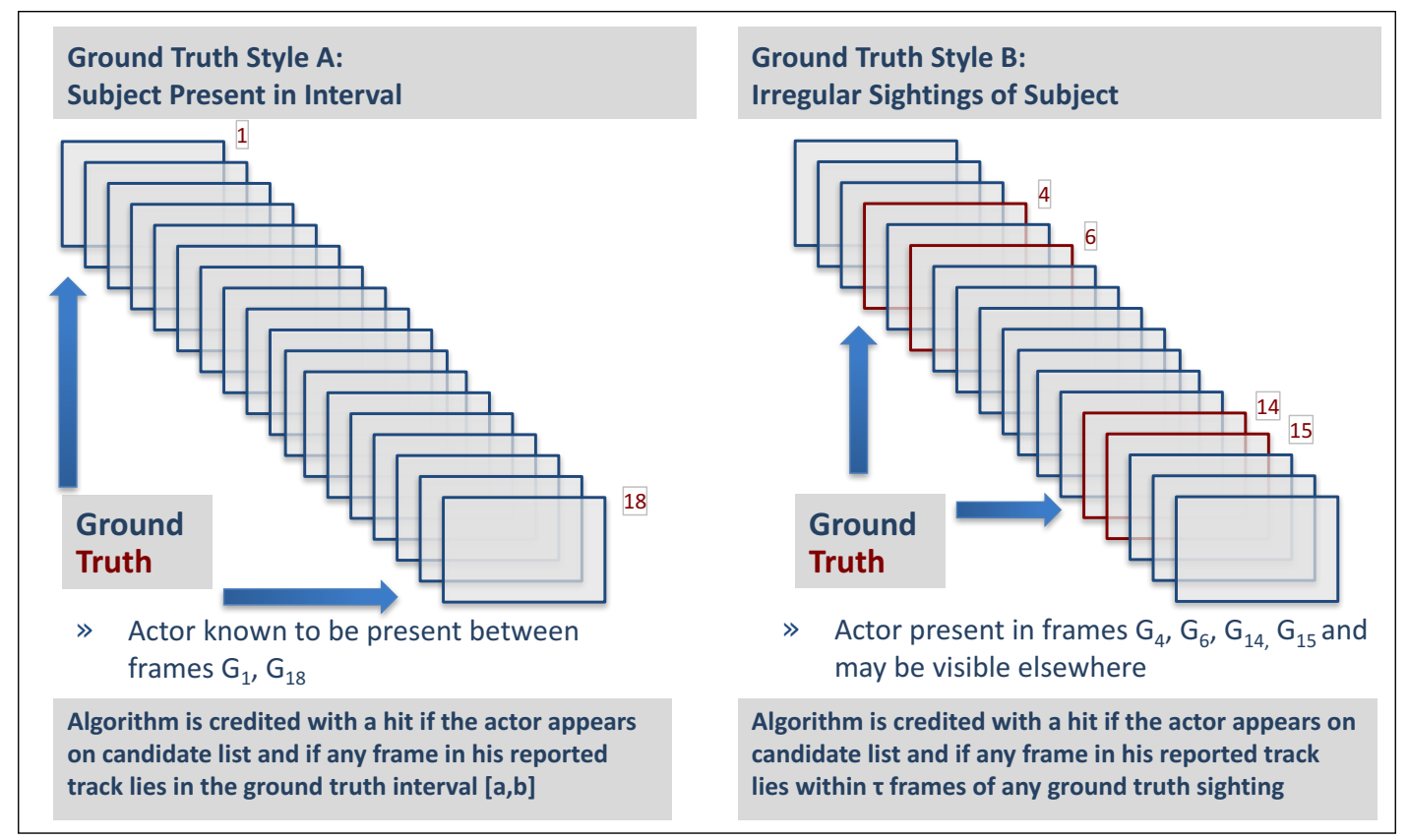

Figure 6: Algorithm evaluation based on ground truth methodology.

that would improve presentation of their faces to the camera. Non-cooperation renders recognition of persons a much more difficult problem compared to applications that involve cooperation of the subjects.

The properties of the data are summarized in Table 3. Further information about the image sets, associated metadata and ground truth are presented alongside the experimental design and the results in the following seven subsections. Each of these gives performance estimates for each evaluated algorithm. The term performance is a generic term covering recognition accuracy, computation duration, and storage requirements. Examples from the image sets are included alongside the recognition results.

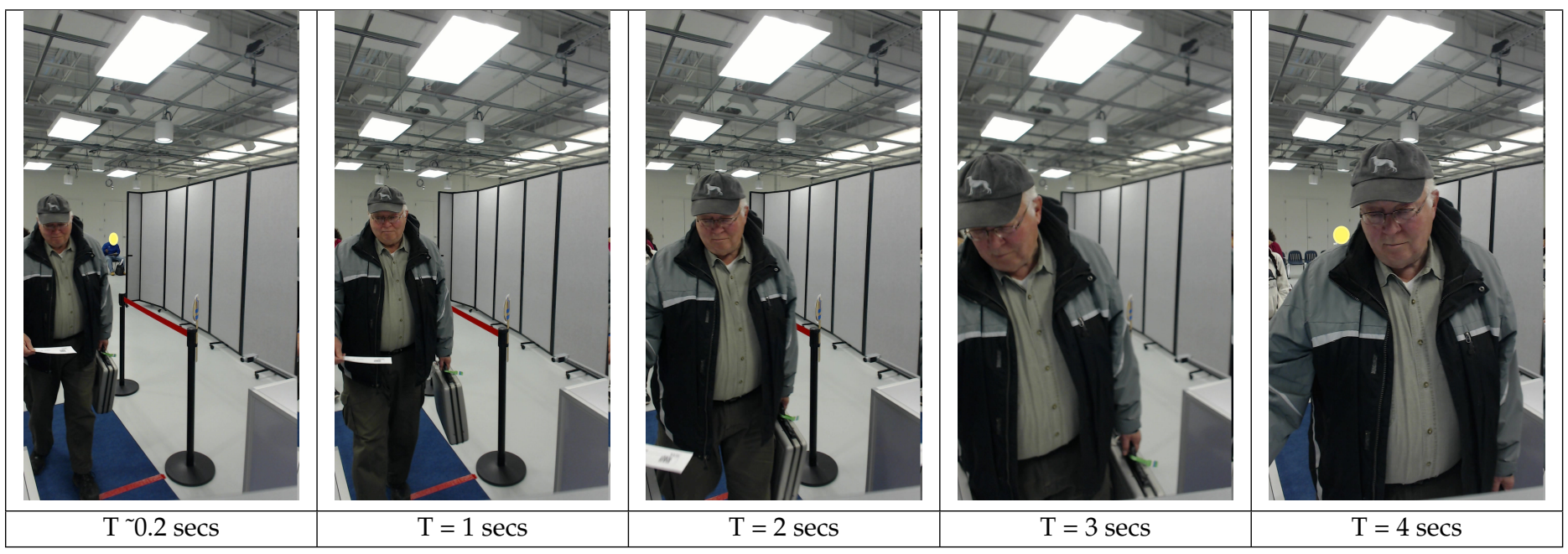

Figure 7: DATASET U: PASSENGER GATE video clip examples. ${ }^{* *}$ The face images in this figure are from the DHS/ S\&T provided AEER dataset. The included subjects consented to release their images in public reports. Subject 79195746 (Perm Granted). Where consent for public release from individuals in the background was not obtained, their faces were masked (yellow circles).

\begin{tabular}{|l|l|l|l|}
\hline \multicolumn{5}{|c|}{ PARTICIPANT KEY } \\
\hline $\mathrm{A}=$ DIGITAL BARRIERS & $\mathrm{E}=$ NEUROTECHNOLOGY & $\mathrm{I}=$ EYEDEA & $\mathrm{M}=$ NEC \\
\hline $\mathrm{B}=$ HBINNO & $\mathrm{F}=$ VAPPLICA & $\mathrm{J}=$ HISIGN & $\mathrm{N}=$ TOSHIBA \\
\hline $\mathrm{C}=$ VIGILANT & $\mathrm{G}=$ MORPHO & $\mathrm{K}=$ COGNITEC & $\mathrm{Q}=$ IMAGUS \\
\hline $\mathrm{D}=$ AYONIX & $\mathrm{H}=$ 3M COGENT & $\mathrm{L}=$ CYBEREXTRUDER & $\mathrm{R}=$ RANK ONE \\
\hline
\end{tabular}

\begin{tabular}{|l|l|l|l|l|l|}
\hline SET & SCENE & CAMERA & SET & SCENE & CAMERA \\
\hline C & PHOTOJOURNALISM & PRO & T & CONCOURSE & PRO \\
\hline J & PASSENGER LOADING & PRO & H & CONCOURSE & PRO \\
\hline P & SPORTS ARENA & CONSUMER & U & CHOKEPOINT & WEBCAM \\
\hline L & LUGGAGE RACK & WEBCAM & & & \\
\hline
\end{tabular}




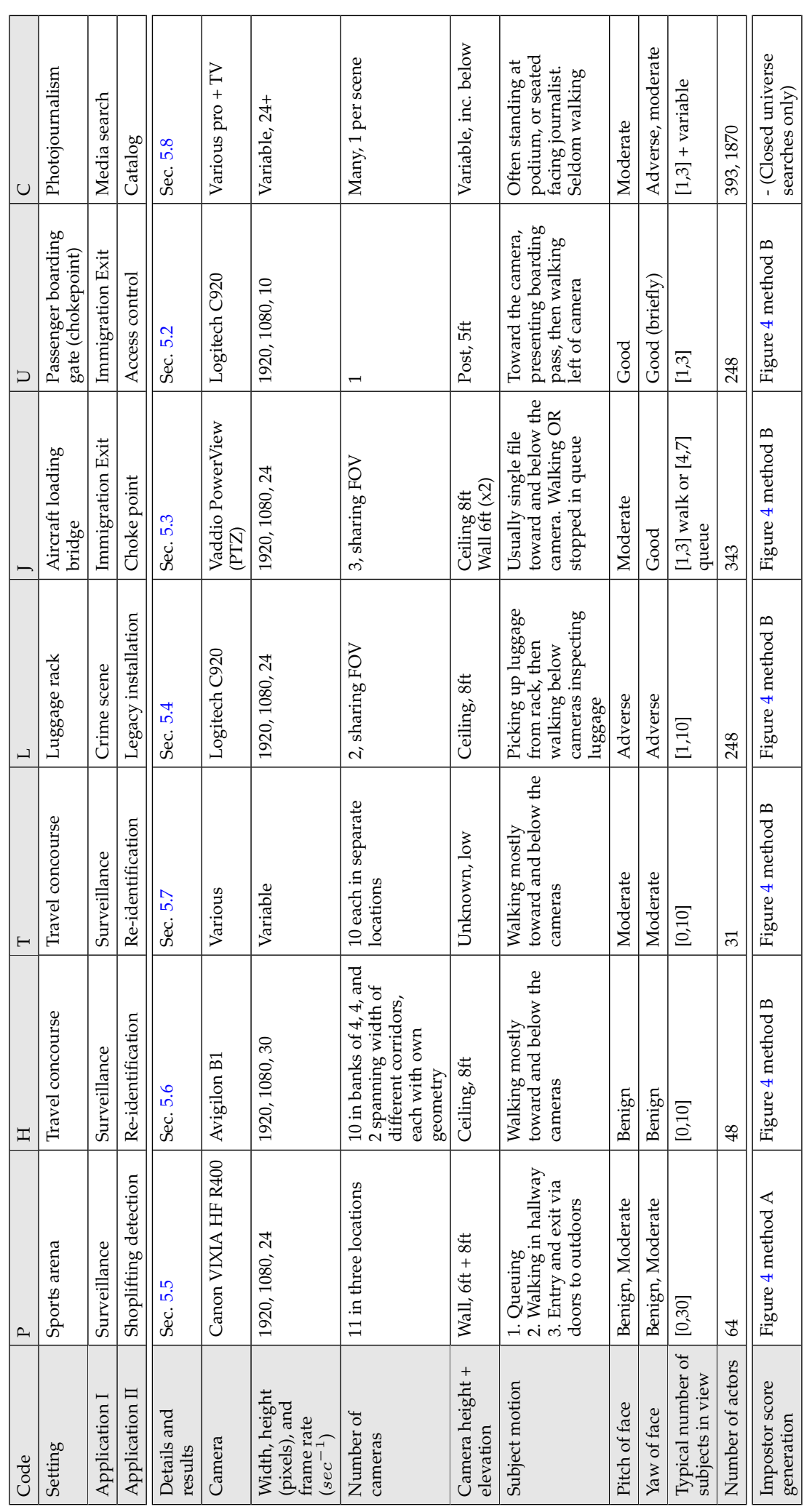

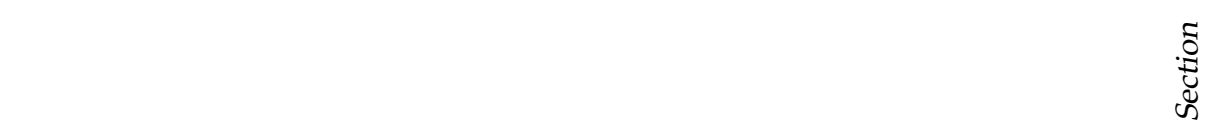




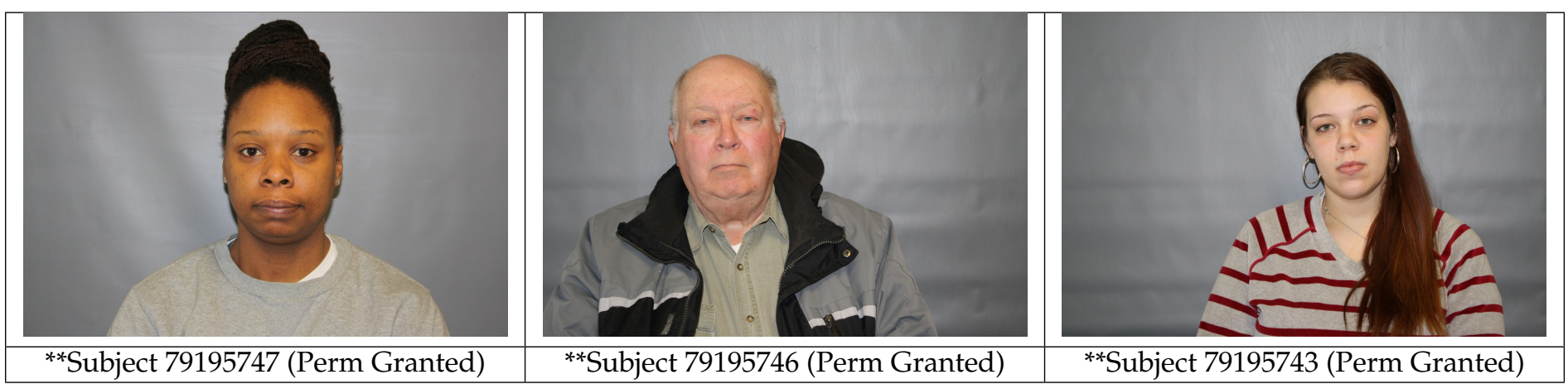

Figure 8: Dataset U: Examples of enrollment images, collected with consumer SLR. ${ }^{*}$ The face images in this figure are from the DHS I S\&T provided AEER dataset. The included subjects consented to release their images in public reports.

\subsection{DATASET U: PASSENGER GATE}

\subsubsection{Overview}

The DATASET U: PASSENGER GATE dataset contains videos of subjects walking toward an aircraft boarding pass reader, using it, then proceeding left across the optical axis passing the camera - see Figure 7 . The subjects were queued in this process, and therefore the faces of other individuals are often present in the scene background.

The imagery was collected in a purely passive mode - the subjects are unaware of the camera, and make no attempt to look at it. The collection, therefore, is entirely non-cooperative. This diverges from the traditional use of biometrics for access control where the boarding pass presentation would form an identity claim and the traveler would be authenticated in a one-to-one process. In this concept of operations a gate might open as the result of this process, something that would require real-time operation. Alternatively, the result of the verification might simply be logged. Here, instead, the video data is searched in oneto-many mode against a dataset of individuals expected to board the aircraft. This single-factor authentication has the advantage of not delaying the existing boarding process at all, but has the cost of elevated recognition error rates over those achievable with one-to-one verification. Note that both of these processes could be conducted with or without cooperation. Even in the case of verification, there might not be any instruction to the traveler to look at the camera.

\begin{tabular}{|l|l|}
\hline Property & Value \\
\hline Camera & Logitech C920 \\
\hline Camera mounting & Attached to display observed by subject \\
\hline Camera height & Approx. 1.75 meters, (5 feet 9 inches) \\
\hline Range to subject & {$[0.7,4]$ meters } \\
\hline Frame rate & $10 \mathrm{sec}^{-1}$ \\
\hline Width & 1080 \\
\hline Height & 1920 \\
\hline Chroma sampling & YUV420 \\
\hline Nominal bitrate & $130 \mathrm{Mb} \mathrm{sec}-1$ \\
\hline Codec & WVC1 (advanced) 0x31435657 \\
\hline
\end{tabular}

Table 4: Key imaging properties for DATASET U: PASSENGER GATE

Videos: The DATASET U: PASSENGER GATE videos are of subjects walking towards and using an aircraft boarding pass reader. Table 4 summarizes key imaging properties and Figure 7 shows examples from the single webcam device that observed this activity.

Enrolled still images: Video clips are matched against a set of enrolled still photographs collected with a consumergrade SLR - see Figure 8. These are in good conformance to the ISO/IEC 19794-5 full frontal image type. These images are enrolled into three galleries of size $N=\{480,4800,48000\}$. These sizes are attained by including high quality frontal

\begin{tabular}{|l|l|l|l|}
\hline \multicolumn{4}{|c|}{ PARTICIPANT KEY } \\
\hline $\mathrm{A}=$ DIGITAL BARRIERS & E = NEUROTECHNOLOGY & I = EYEDEA & $\mathrm{M}=$ NEC \\
\hline $\mathrm{B}=$ HBINNO & $\mathrm{F}=$ VAPPLICA & $\mathrm{J}=$ HISIGN & $\mathrm{N}=$ TOSHIBA \\
\hline $\mathrm{C}=$ VIGILANT & $\mathrm{G}=$ MORPHO & $\mathrm{K}=$ COGNITEC & $\mathrm{Q}=$ IMAGUS \\
\hline $\mathrm{D}=$ AYONIX & $\mathrm{H}=$ 3M COGENT & $\mathrm{L}=$ CYBEREXTRUDER & $\mathrm{R}=$ RANK ONE \\
\hline
\end{tabular}

\begin{tabular}{|l|l|l|l|l|l|}
\hline SET & SCENE & CAMERA & SET & SCENE & CAMERA \\
\hline C & PHOTOJOURNALISM & PRO & T & CONCOURSE & PRO \\
\hline J & PASSENGER LOADING & PRO & H & CONCOURSE & PRO \\
\hline P & SPORTS ARENA & CONSUMER & U & CHOKEPOINT & WEBCAM \\
\hline L & LUGGAGE RACK & WEBCAM & & & \\
\hline
\end{tabular}




\begin{tabular}{|l|l|}
\hline Quantity & Value or description \\
\hline Mode & Video search to still enrollment \\
\hline Number of actors & 248 \\
\hline Number of non-actors & 0 \\
\hline Number of cameras & 1 \\
\hline Video duration with actors & 18.4 minutes \\
\hline Video duration no actors & 0 \\
\hline Subject motion & Toward and then to left of camera \\
\hline Number of clips & 248, mean length 4.5 seconds \\
\hline Clip sequencing & Main person in clip $n$ is in background in clip $n-1$ \\
\hline Clip duration (frames) & Median $43 ;$ Min $18 ;$ Q25 37; Q75 48; Max 102 \\
\hline Number of enrolled subjects & $480,4800,48000$ \\
\hline Number of enrolled stills & 1 per subject \\
\hline Properties of enrolled stills & Frontal, close ICAO compliance; Mean IOD 106 pixels \\
\hline FNIR estimation & Actors present video vs. enrolled gallery \\
\hline FPIR estimation & Actors present video vs. separate non-actor gallery \\
\hline Candidate list length & 20 \\
\hline Number of persons in FOV & {$[0,3]$ usually; one dominant in foreground } \\
\hline Video ground truth & Style A: See Figure 6 \\
\hline
\end{tabular}

Table 5: Key experimental design the DATASET U: PASSENGER GATE results.

portrait photographs from a disjoint background population. Exactly one image is enrolled for each person.

Experimental Design: Mated scores are generated by searching 248 video clips against the three enrolled dataset of still face images of subjects known to be in the search videos. Nonmated scores are collected by comparing the same 248 video clips against three enrollment datasets known not to contain actor images. These sets, which contain $\mathrm{N}=$ $\{480,4800,48000\}$ frontal images, are termed the global nonmated enrollment dataset, and are used to generate nonmated comparison scores for other datasets as well. A limitation of this approach is that by searching video of only 248 individuals we cannot calibrate thresholds that would yield low false positive identification rates (e.g. FPIR $=10^{-5}$ ). To do that we'd need to run many more searches from many more people, something we do later for Dataset $P$.

Key experimental design details are summarized in Table 5.

\subsubsection{Accuracy}

Results for the DATASET U: PASSENGER GATE set are presented exhaustively in Tables 6 - 8, one for each enrolled population size. These tabulate false negative identification rates, $\operatorname{FNIR}(N, R, T)$, in the two special cases, one relevant to investigations, $\operatorname{FNIR}(N, 1,0)$, and the other to high volume identification, $\operatorname{FNIR}(N, L, T)$ where $\mathrm{T}$ is set to realize a fixed number of false positives, $\operatorname{NFP}(T)$ over all video clips. Extracts from these are then graphed in Figures 9 - 11.

The notable results are:

Absolute accuracy: The most accurate algorithms are those submitted by participant M (M30V, M31V, M32V). At the strictest threshold M30V achieves an FNIR of 0.056. The detailed interpretation of this is important. In an access control context, it says that when 248 subjects pass through a chokepoint, while executing their document scanning task, we expect to correctly identify $94.4 \%$ of those subjects in a gallery of size $N=480$, without any explicit cooperation from them, while only producing 1 false positive. What is a false positive in this context? It is the failure to correctly reject an unauthorized (unenrolled) subject who attempts to gain access. Given that such an impostor might be rare, it may be tenable from a security perspective, to lower the threshold such that 10 false positives out of 248 people passing the chokepoint was acceptable. In that case, the same algorithm would then

\begin{tabular}{|l|l|l|l|}
\hline \multicolumn{4}{|c|}{ PARTICIPANT KEY } \\
\hline A = DIGITAL BARRIERS & E = NEUROTECHNOLOGY & I = EYEDEA & $\mathrm{M}=$ NEC \\
\hline B = HBINNO & F = VAPPLICA & J = HISIGN & N = TOSHIBA \\
\hline C = VIGILANT & G = MORPHO & K = COGNITEC & $\mathrm{Q}=$ IMAGUS \\
\hline $\mathrm{D}=$ AYONIX & $\mathrm{H}=$ 3M COGENT & $\mathrm{L}=$ CYBEREXTRUDER & $\mathrm{R}=$ RANK ONE \\
\hline
\end{tabular}

\begin{tabular}{|l|l|l|l|l|l|}
\hline SET & SCENE & CAMERA & SET & SCENE & CAMERA \\
\hline C & PHOTOJOURNALISM & PRO & T & CONCOURSE & PRO \\
\hline J & PASSENGER LOADING & PRO & H & CONCOURSE & PRO \\
\hline P & SPORTS ARENA & CONSUMER & U & CHOKEPOINT & WEBCAM \\
\hline L & LUGGAGE RACK & WEBCAM & & & \\
\hline
\end{tabular}


correctly identify $96.4 \%$ of legitimate subjects.

Breadth of capability: Despite the favorable geometric and optical configuration of the installation, face recognition accuracy varies widely across developers. Four algorithms miss more than $90 \%$ of subjects at $\operatorname{NFP}(T)=10$, while nineteen algorithms achieve an FNIR below 25\%, eleven achieve an FNIR below 15\% at the same decision threshold, and five achieve an FNIR below 10\% (M30V, M31V, M32V, G31V, G32V). On this basis, the marketplace capability is not broad.

Rank based accuracy: If for some reason, video from this geometrical and optical configuration were occasionally used in forensic searches, such as a human trafficking investigation, accuracy is better, and many more algorithms offer useful accuracy. Thus, even at $\mathrm{N}=48000$, the best accuracy is a rank-20 hit rate of $99.2 \%(\mathrm{M} 31 \mathrm{~V})$ with 18 algorithms giving better than $90 \%$.

Effect of threshold: Figure 9 shows accuracy for $\mathrm{N}=480$, at three decision thresholds corresponding to false positives counts of 1, 10, and 100. The latter two numbers are quite high: Ten false positives means that about one in every twenty-five (10/248) video clips would yield a false positive - the incorrect identification of a person with the enrolled subject. Because there is a tradeoff between the FNIR and the number of false positives, decision thresholds that elicit the fewest false positives, here 1, produce the highest $\operatorname{FNIR}(N, L, T)$.

Figure 10 plots $\operatorname{FNIR}(N, L, T)$ against the count of false positives. This plot is related to the error tradeoff characteristic that typically appears in biometric performance reports. It differs in that the x-axis is a count of false positives rather than a rate because the numbers of persons appearing in video imagery is (generally) not known. Such a plot is useful in that it supports cost benefit decisions: The y-axis, $\operatorname{FNIR}(N, L, T)$, is related to the benefit and the x-axis, $\operatorname{NFP}(T)$, drives the costs associated with subsequent consequences and resolution of false positives, typically via human adjudication and intervention.

Algorithm tuning: Some algorithms are configured toward giving better forensic investigational mode accuracy than in the high-threshold identification mode. This design feature is typical in biometrics, and suggests algorithm variants should be procured or parameterized properly, to emphasize discrimination between faces in large populations vs. invariance to facial appearance. Some algorithms are more robust to relaxation of the decision threshold than others. This sometimes makes it difficult to say one algorithm is more accurate than another in any absolute sense. For example, when $N=480$, G32V and H30V produce similar FNIRs at FP=100 (0.048 vs. 0.044 respectively), but at $\mathrm{FP}=10, \mathrm{G} 32 \mathrm{~V}$ achieves a much lower FNIR (0.056 vs. 0.137). Similarly, at FP=100, N32V achieves a lower FNIR than K31V (0.065 vs. 0.117) but at FP=10, K31V achieves a lower FNIR (0.145 vs. 0.185). The most accurate algorithm, M31V, achieves an FNIR at least as low as every other algorithm at all three decision thresholds.

Scaling to large gallery sizes: Figure 11 shows how FNIR is affected as the enrolled population increases from $\mathrm{N}=480$ to $\mathrm{N}=48000$. As $\mathrm{N}$ increases, false positives occur more frequently and they displace rank one hits in mated searches, and necessitate higher decision thresholds to suppress false positives in non-mated searches. Generally, increasing N leads to elevated FNIR miss rate. For M30V, the FNIR(N, L, T10) increases from 0.032 to 0.097, roughly a factor of 3 increase for a 100-fold increase in the size of the gallery (from 480 to 48,000 ). For most algorithms, the FNIR tends to increase linearly with the log of the gallery size, paralleling the behavior of many still-face recognition algorithms [22,23]. This dependence is benign and is the fundamental basis for the utility of face recognition in large population applications.

\begin{tabular}{|l|l|l|l|}
\hline \multicolumn{5}{|c|}{ PARTICIPANT KEY } \\
\hline $\mathrm{A}=$ DIGITAL BARRIERS & $\mathrm{E}=$ NEUROTECHNOLOGY & $\mathrm{I}=$ EYEDEA & $\mathrm{M}=$ NEC \\
\hline $\mathrm{B}=$ HBINNO & $\mathrm{F}=$ VAPPLICA & $\mathrm{J}=$ HISIGN & $\mathrm{N}=$ TOSHIBA \\
\hline $\mathrm{C}=$ VIGILANT & $\mathrm{G}=$ MORPHO & $\mathrm{K}=$ COGNITEC & $\mathrm{Q}=$ IMAGUS \\
\hline $\mathrm{D}=$ AYONIX & $\mathrm{H}=3 \mathrm{M}$ COGENT & $\mathrm{L}=$ CYBEREXTRUDER & $\mathrm{R}=$ RANK ONE \\
\hline
\end{tabular}

\begin{tabular}{|l|l|l|l|l|l|}
\hline SET & SCENE & CAMERA & SET & SCENE & CAMERA \\
\hline C & PHOTOJOURNALISM & PRO & T & CONCOURSE & PRO \\
\hline J & PASSENGER LOADING & PRO & H & CONCOURSE & PRO \\
\hline P & SPORTS ARENA & CONSUMER & U & CHOKEPOINT & WEBCAM \\
\hline L & LUGGAGE RACK & WEBCAM & & & \\
\hline
\end{tabular}


The effect of enrolled gallery size is evident also in Figure 10, which replots the tabulated FNIR values. The expected behavior from binomial models, would be for the lines to be parallel and evenly spaced indicating that FNIR is conserved when a ten-fold increase in the gallery size is accompanied by a ten-fold increase in the number of tolerable false positives. Visually this applies only for some algorithms (e.g. I30V, J3xV).

Note that algorithm G30V and the four algorithms from provider $\mathrm{N}$ give better accuracy when $\mathrm{N}=4800$ than at $\mathrm{N}$ $=480$. This appears when the lines in Figure 10 cross, as NFP $\rightarrow 1$. This effect is unexpected. It may arise because gallery score normalization is more effective with larger N. Score normalization schemes are often used to stabilize the nonmate distribution. The rank-one miss rates do increase with $\mathrm{N}$ as expected.

Relevance to an access control application: The consequences of false negatives and positives differ by application. If this was a positive access control application using one-to-many identification to permit entry to a building, for example, then a false positive would correspond to an incorrect admission. This would occur if an unauthorized user matched any of the enrolled entries. Given the dataset and the results for it, can we conclude that accuracy would be fit-for-purpose? An access control application would require false positive identification rates to be somewhat lower than achieved here, i.e. FPIR $<1 / 248$. Our best miss rate at this kind of false positive identification rate - the yellow column in Table 6 - is around 6\%. However this result is pessimistic in that "white-list" applications have subjects who can engage the face camera in multiple cooperative attempts. Note that impostors, too, can make repeated attempts at recognition, unless some mechanism is implemented to impede that. The use of face recognition without an identity claim is an example of single-factor authentication and is therefore inherently weak from a security perspective. Acknowledging this, the value of video-based recognition here is in expediting two factor access control by using one-to-many identification of a subject as she approaches a document reader. This has potential to expedite the process, by doing face capture and feature extraction, prior to presentation of the token. Recognition too could be done as a one-to-many search of a database, if available, or against biometric data read from the identity token.

Relevance to an immigration exit application: If this was an immigration exit application using one-to-many identification to record biometric exit of in-scope visa holders boarding at an international departure gate, then the following categories of error need to be considered and addressed

- Failure to enrol: If an in-scope traveler's reference photograph is not enrolled into the gallery, then this counts toward the failure to enrol rate (FTE). This could occur because the reference photo was simply unavailable, or was of such poor quality that the algorithm could not, or would not, produce a template from it. This would essentially be an additive increment to $\operatorname{FNIR}(N, L, T)$. In this report, $\mathrm{FTE}=0$, by design. In operations, it may occur, for example when a new employee's photograph is not enrolled into an access control system.

- Failure to acquire: If an in-scope traveler is not detected by the face recognition algorithm, then the result is effectively a false negative. This could occur, for example, if the camera simply didn't cover the proper volume, or because the person's face was occluded by another's. If an explicit measurement of the failure to acquire rate, FTA, was available it could be combined with a false negative identification rate measured over a population appearing as intended, to give an overall statement of false negative error

$$
\text { FNIR }_{\text {OVERALL }}=\text { FTA }+(1-\text { FTA }) \text { FNIR MEASURED }
$$

The point is that FTA is the proportion of travelers who would not be recognized even with a perfect recogni-

\begin{tabular}{|l|l|l|l|}
\hline \multicolumn{5}{|c|}{ PARTICIPANT KEY } \\
\hline $\mathrm{A}=$ DIGITAL BARRIERS & $\mathrm{E}=$ NEUROTECHNOLOGY & $\mathrm{I}=$ EYEDEA & $\mathrm{M}=$ NEC \\
\hline $\mathrm{B}=$ HBINNO & $\mathrm{F}=$ VAPPLICA & $\mathrm{J}=$ HISIGN & $\mathrm{N}=$ TOSHIBA \\
\hline $\mathrm{C}=$ VIGILANT & $\mathrm{G}=$ MORPHO & $\mathrm{K}=$ COGNITEC & $\mathrm{Q}=$ IMAGUS \\
\hline $\mathrm{D}=$ AYONIX & $\mathrm{H}=$ 3M COGENT & $\mathrm{L}=$ CYBEREXTRUDER & $\mathrm{R}=$ RANK ONE \\
\hline
\end{tabular}

\begin{tabular}{|l|l|l|l|l|l|}
\hline SET & SCENE & CAMERA & SET & SCENE & CAMERA \\
\hline C & PHOTOJOURNALISM & PRO & T & CONCOURSE & PRO \\
\hline J & PASSENGER LOADING & PRO & H & CONCOURSE & PRO \\
\hline P & SPORTS ARENA & CONSUMER & U & CHOKEPOINT & WEBCAM \\
\hline L & LUGGAGE RACK & WEBCAM & & & \\
\hline
\end{tabular}


tion algorithm.

- False negative: If an algorithm does not identify an in-scope traveler against his enrollment template, then this counts toward elevated false negative identification rate, $\operatorname{FNIR}(N, R, T)$. This is a common occurence, and is the primary subject of this report.

- False positive: A false positive would occur when video from traveler A was incorrectly associated with an enrolled person, B, who is expected to board the aircraft. Person A could also be a) an in-scope traveler who just happens to match $B$ rather than his own enrollment, or b) a traveler who is not enrolled at all. In this case, person A could instead be colluding with an enrolled subject, B, and trying to specifically impersonate him to record exit. This is very unlikely to be successful - probability $\sim 1 / N$ without any effort, but higher with a dedicated presentation attack e.g. by using a sibling, or a face mask.

Further improving accuracy: With further trials it may be possible to improve accuracy. This could be achieved by improving temporal resolution (30 frames per second instead of 10), and (expensively) by adding another camera. However, it may also be possible by refining the position of the camera, in particular by colocating the camera with the boarding pass reader. While this would yield frontal and higher resolution frames, it may also impart some advserse distortion associated with the camera being too close to the subject. The key point here is that prospective deployers must engage in a deliberate optical, mechanical and environmental design effort.

\subsubsection{Resolution}

Optical resolution is highly influential on face recognition as it is necessary to resolve features that afford discrimination. Resolution in the recorded imagery is afforded by proximity of the camera to the subject, by design of specific optical properties of the camera, by lack of motion blur, and by benign application of modern compression algorithms, particularly in video.

We have no formal resolution measurements for the imagery used in FIVE. The only indicator we have is interocular distance (IOD) i.e. the distance between the eyes, as reported by face detection algorithm. This is measure of spatial sampling rate, and is a weak proxy for optical resolution (because low resolution images can be interpolated to produce high spatial sampling rates). Nevertheless it is a useful design parameter in face recognition, because it is assumed that the optical and compression specifications have been well designed.

For DATASET U: PASSENGER GATE Table 9 shows statistics of IOD reported while tracking individuals in video. It also reports track length statistics. The notable observations are:

Track lengths: Algorithms vary in the lengths of the tracks they report. Some algorithms (R, C, F) limit feature extraction to fewer than 7 frames corresponding to 0.7 seconds. Others consider longer tracks (K31V, G31V, G32V, $\mathrm{N} 3 x \mathrm{~V}, \mathrm{E} 30 \mathrm{~V}$ ) with extent approaching 30 frames (3 seconds).

Resolution: Algorithms vary in which part of the video clips they elect to use. The tabulated IOD values are averages of the minimum, mean, and maximum IOD reported for each track. The M3xV algorithms have tracks with mean IOD of 40 pixels. The J, D, R, and L algorithms extract information from subjects with mean IOD above 75 pixels. Thus, refering to Figure 7, some algorithms focus on subjects when they are relatively far from the camera, and others when they are close. In this dataset, the faces of subjects close to the camera usually exhibit larger yaw angles.

\begin{tabular}{|l|l|l|l|}
\hline \multicolumn{5}{|c|}{ PARTICIPANT KEY } \\
\hline $\mathrm{A}=$ DIGITAL BARRIERS & $\mathrm{E}=$ NEUROTECHNOLOGY & $\mathrm{I}=$ EYEDEA & $\mathrm{M}=$ NEC \\
\hline $\mathrm{B}=$ HBINNO & $\mathrm{F}=$ VAPPLICA & $\mathrm{J}=$ HISIGN & $\mathrm{N}=$ TOSHIBA \\
\hline $\mathrm{C}=$ VIGILANT & $\mathrm{G}=$ MORPHO & $\mathrm{K}=$ COGNITEC & $\mathrm{Q}=$ IMAGUS \\
\hline $\mathrm{D}=$ AYONIX & $\mathrm{H}=$ 3M COGENT & $\mathrm{L}=$ CYBEREXTRUDER & $\mathrm{R}=$ RANK ONE \\
\hline
\end{tabular}

\begin{tabular}{|l|l|l|l|l|l|}
\hline SET & SCENE & CAMERA & SET & SCENE & CAMERA \\
\hline C & PHOTOJOURNALISM & PRO & T & CONCOURSE & PRO \\
\hline J & PASSENGER LOADING & PRO & H & CONCOURSE & PRO \\
\hline P & SPORTS ARENA & CONSUMER & U & CHOKEPOINT & WEBCAM \\
\hline L & LUGGAGE RACK & WEBCAM & & & \\
\hline
\end{tabular}




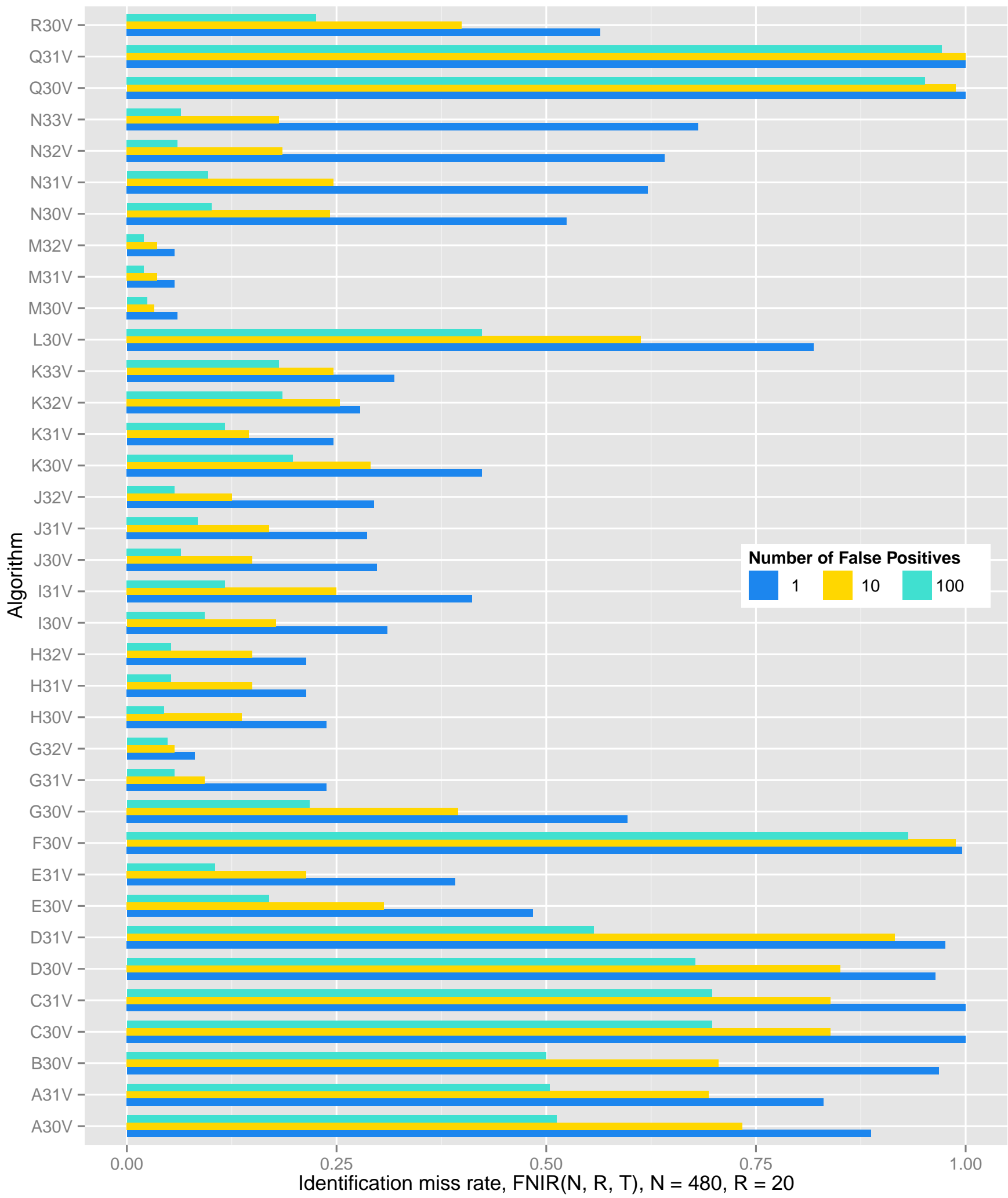

Figure 9: For DATASET U: PASSENGER GATE with $N=480$ subject enrolled, the bars show FNIR $(N, L, T)$ for three different decision thresholds, T corresponding to 1, 10 and 100 false positives. High thresholds are necessary in applications to match the number of false positives to the human labor availability needed for their adjudication. False positive outcomes increase linearly with the number of faces appearing in video.

\begin{tabular}{|l|l|l|l|}
\hline \multicolumn{4}{|c|}{ PARTICIPANT KEY } \\
\hline $\mathrm{A}=$ DIGITAL BARRIERS & E = NEUROTECHNOLOGY & $\mathrm{I}=$ EYEDEA & $\mathrm{M}=$ NEC \\
\hline $\mathrm{B}=$ HBINNO & F = VAPPLICA & $\mathrm{J}=$ HISIGN & $\mathrm{N}=$ TOSHIBA \\
\hline $\mathrm{C}=$ VIGILANT & $\mathrm{G}=$ MORPHO & K = COGNITEC & $\mathrm{Q}=$ IMAGUS \\
\hline $\mathrm{D}=$ AYONIX & $\mathrm{H}=$ 3M COGENT & $\mathrm{L}=$ CYBEREXTRUDER & $\mathrm{R}=$ RANK ONE \\
\hline
\end{tabular}

\begin{tabular}{|l|l|l|l|l|l|}
\hline SET & SCENE & CAMERA & SET & SCENE & CAMERA \\
\hline C & PHOTOJOURNALISM & PRO & T & CONCOURSE & PRO \\
\hline J & PASSENGER LOADING & PRO & H & CONCOURSE & PRO \\
\hline P & SPORTS ARENA & CONSUMER & U & CHOKEPOINT & WEBCAM \\
\hline L & LUGGAGE RACK & WEBCAM & & & \\
\hline
\end{tabular}




\begin{tabular}{|c|c|c|c|c|c|c|c|c|c|c|c|c|c|}
\hline \multirow{3}{*}{$\begin{array}{l}\mathrm{N}=480 \\
\mathrm{ALG}\end{array}$} & \multicolumn{2}{|c|}{ NUM ACTORS 248} & \multicolumn{2}{|c|}{ NUM FEEDS 1} & \multicolumn{3}{|c|}{ NUM CLIPS 248} & \multicolumn{3}{|c|}{ NUM FRAMES 11012} & \multicolumn{3}{|c|}{ NUM MINUTES 18.4} \\
\hline & \multirow{2}{*}{$\begin{array}{r}\text { DETECTIONS } \\
\text { NUM } \\
\end{array}$} & \multicolumn{6}{|c|}{ THRESHOLD BASED AUTO WATCHLISTS } & \multicolumn{6}{|c|}{ RANK BASED FORENSIC CASES } \\
\hline & & \multicolumn{2}{|c|}{$\operatorname{FNIR}(\mathrm{T}), \operatorname{FP}(\mathrm{T})=1$} & \multicolumn{2}{|c|}{$\operatorname{FNIR}(\mathrm{T}), \operatorname{FP}(\mathrm{T})=10$} & \multicolumn{2}{|c|}{$\operatorname{FNIR}(\mathrm{T}), \mathrm{FP}(\mathrm{T})=100$} & \multicolumn{2}{|c|}{$\operatorname{FNIR}(\mathrm{R}=1, \mathrm{~T}=0)$} & \multicolumn{2}{|c|}{$\operatorname{FNIR}(\mathrm{R}=5, \mathrm{~T}=0)$} & \multicolumn{2}{|c|}{$\operatorname{FNIR}(\mathrm{R}=20, \mathrm{~T}=0)$} \\
\hline A30V & 1345 & 0.887 & 28 & 0.734 & 29 & 0.512 & 29 & 0.290 & 28 & 0.121 & 27 & 0.056 & 24 \\
\hline A31V & 1345 & 0.831 & 27 & 0.694 & 27 & 0.504 & 28 & 0.282 & 27 & 0.117 & 26 & 0.056 & 23 \\
\hline B30V & 714 & 0.968 & 32 & 0.706 & 28 & 0.500 & 27 & 0.274 & 26 & 0.153 & 30 & 0.060 & 25 \\
\hline C30V & 2364 & 0.903 & 29 & 0.839 & 31 & 0.698 & 33 & 0.423 & 33 & 0.331 & 32 & 0.290 & 33 \\
\hline C31V & 2661 & 0.907 & 30 & 0.839 & 30 & 0.698 & 32 & 0.419 & 32 & 0.335 & 33 & 0.282 & 32 \\
\hline D30V & 2004 & 0.964 & 31 & 0.851 & 32 & 0.677 & 31 & 0.347 & 31 & 0.145 & 28 & 0.081 & 28 \\
\hline D31V & 638 & 0.976 & 33 & 0.915 & 33 & 0.556 & 30 & 0.315 & 29 & 0.145 & 29 & 0.093 & 29 \\
\hline E30V & 743 & 0.484 & 19 & 0.306 & 23 & 0.169 & 20 & 0.093 & 22 & 0.065 & 22 & 0.036 & 20 \\
\hline E31V & 743 & 0.391 & 16 & 0.214 & 16 & 0.105 & 17 & 0.065 & 16 & 0.044 & 19 & 0.032 & 19 \\
\hline F30V & 1502 & 0.996 & 34 & 0.988 & 35 & 0.931 & 34 & 0.875 & 34 & 0.710 & 34 & 0.476 & 34 \\
\hline G30V & 493 & 0.597 & 22 & 0.395 & 24 & 0.218 & 24 & 0.173 & 25 & 0.113 & 24 & 0.105 & 30 \\
\hline G31V & 691 & 0.238 & 8 & 0.093 & 5 & 0.056 & 8 & 0.056 & 15 & 0.044 & 20 & 0.040 & 21 \\
\hline G32V & 691 & 0.081 & 4 & 0.056 & 4 & 0.048 & 5 & 0.056 & 14 & 0.048 & 21 & 0.044 & 22 \\
\hline $\mathrm{H} 30 \mathrm{~V}$ & 668 & 0.238 & 7 & 0.137 & 7 & 0.044 & 4 & 0.036 & 4 & 0.012 & 4 & 0.012 & 6 \\
\hline H31V & 668 & 0.214 & 5 & 0.149 & 9 & 0.052 & 6 & 0.040 & 5 & 0.020 & 5 & 0.012 & 4 \\
\hline $\mathrm{H} 32 \mathrm{~V}$ & 668 & 0.214 & 6 & 0.149 & 10 & 0.052 & 7 & 0.040 & 7 & 0.020 & 6 & 0.012 & 5 \\
\hline I31V & 1382 & 0.411 & 17 & 0.250 & 20 & 0.117 & 19 & 0.077 & 20 & 0.040 & 18 & 0.028 & 17 \\
\hline $\mathrm{J} 30 \mathrm{~V}$ & 441 & 0.298 & 13 & 0.149 & 11 & 0.065 & 12 & 0.048 & 10 & 0.024 & 8 & 0.012 & 8 \\
\hline J31V & 441 & 0.286 & 11 & 0.169 & 12 & 0.085 & 13 & 0.069 & 18 & 0.036 & 13 & 0.012 & 7 \\
\hline $\mathrm{J} 32 \mathrm{~V}$ & 441 & 0.294 & 12 & 0.125 & 6 & 0.056 & 9 & 0.044 & 9 & 0.024 & 10 & 0.016 & 10 \\
\hline K30V & 1547 & 0.423 & 18 & 0.290 & 22 & 0.198 & 23 & 0.052 & 12 & 0.040 & 17 & 0.020 & 11 \\
\hline K31V & 941 & 0.246 & 9 & 0.145 & 8 & 0.117 & 18 & 0.052 & 11 & 0.040 & 16 & 0.028 & 16 \\
\hline K32V & 757 & 0.278 & 10 & 0.254 & 21 & 0.185 & 22 & 0.161 & 24 & 0.113 & 25 & 0.077 & 27 \\
\hline K33V & 779 & 0.319 & 15 & 0.246 & 18 & 0.181 & 21 & 0.149 & 23 & 0.109 & 23 & 0.077 & 26 \\
\hline L30V & 526 & 0.819 & 26 & 0.613 & 26 & 0.423 & 26 & 0.339 & 30 & 0.238 & 31 & 0.194 & 31 \\
\hline M30V & 934 & 0.060 & 3 & 0.032 & 1 & 0.024 & 3 & 0.016 & 3 & 0.004 & 2 & 0.004 & 3 \\
\hline M31V & 934 & 0.056 & 2 & 0.036 & 3 & 0.020 & 2 & 0.016 & 2 & 0.004 & 1 & 0.004 & 2 \\
\hline M32V & 934 & 0.056 & 1 & 0.036 & 2 & 0.020 & 1 & 0.008 & 1 & 0.008 & 3 & 0.004 & 1 \\
\hline N30V & 608 & 0.524 & 20 & 0.242 & 17 & 0.101 & 16 & 0.065 & 17 & 0.036 & 14 & 0.020 & 13 \\
\hline N31V & 608 & 0.621 & 23 & 0.246 & 19 & 0.097 & 15 & 0.052 & 13 & 0.028 & 11 & 0.016 & 9 \\
\hline N32V & 608 & 0.641 & 24 & 0.185 & 15 & 0.060 & 10 & 0.040 & 8 & 0.024 & 9 & 0.020 & 12 \\
\hline N33V & 608 & 0.681 & 25 & 0.181 & 14 & 0.065 & 11 & 0.040 & 6 & 0.024 & 7 & 0.024 & 14 \\
\hline Q30V & 501 & 1.000 & 35 & 0.988 & 34 & 0.952 & 35 & 0.907 & 35 & 0.819 & 35 & 0.714 & 35 \\
\hline Q31V & 501 & 1.000 & 36 & 1.000 & 36 & 0.972 & 36 & 0.952 & 36 & 0.859 & 36 & 0.742 & 36 \\
\hline
\end{tabular}

Table 6: For the DATASET U: PASSENGER GATE installation, with 480 subjects enrolled with a frontal still, the values are identificationmode FNIR(T) for each algorithm at three different decision thresholds corresponding to false positive counts of 1, 10, 100, and investigation-mode FNIR(R) for ranks 1, 5, 20. Each value is accompanied by an integer ranking across all algorithms. The shaded columns indicates the most important metric to watchlist applications. The green shaded cells indicates the most accurate algorithm. Caution: The last column give optimistically low error rates per the arguments of section 4.4.

\begin{tabular}{|c|c|c|c|c|c|c|c|c|c|}
\hline \multicolumn{4}{|c|}{ PARTICIPANT KEY } & SET & SCENE & CAMERA & SET & SCENE & CAMERA \\
\hline $\mathrm{A}=$ DIGITAL BARRIERS & $\mathrm{E}=$ NEUROTECHNOLOGY & $\mathrm{I}=\mathrm{EYEDEA}$ & $M=N E C$ & C & PHOTOJOURNALISM & PRO & $\mathrm{T}$ & CONCOURSE & PRO \\
\hline $\mathrm{B}=\mathrm{HBINNO}$ & $\mathrm{F}=$ VAPPLICA & $\mathrm{J}=\mathrm{HISIGN}$ & $\mathrm{N}=$ TOSHIBA & $\mathrm{J}$ & PASSENGER LOADING & PRO & $\mathrm{H}$ & CONCOURSE & PRO \\
\hline $\mathrm{C}=$ VIGILANT & $\mathrm{G}=\mathrm{MORPHO}$ & $\mathrm{K}=$ COGNITEC & $\mathrm{Q}=$ IMAGUS & $\mathrm{P}$ & SPORTS ARENA & CONSUMER & U & CHOKEPOINT & WEBCAM \\
\hline $\mathrm{D}=\mathrm{AYONIX}$ & $\mathrm{H}=3 \mathrm{M}$ COGENT & $\mathrm{L}=$ CYBEREXTRUDER & $\mathrm{R}=\mathrm{RANK}$ ONE & $\mathrm{L}$ & LUGGAGE RACK & WEBCAM & & & \\
\hline
\end{tabular}




\begin{tabular}{|c|c|c|c|c|c|c|c|c|c|c|c|c|c|}
\hline \multirow{4}{*}{$\begin{array}{l}\mathrm{N}=4800 \\
\text { ALG } \\
\text { A30V }\end{array}$} & \multicolumn{2}{|c|}{ NUM ACTORS 248} & \multicolumn{2}{|c|}{ NUM FEEDS 1} & \multicolumn{3}{|c|}{ NUM CLIPS 248} & \multicolumn{3}{|c|}{ NUM FRAMES 11012} & \multicolumn{3}{|c|}{ NUM MINUTES 18.4} \\
\hline & \multirow{2}{*}{$\begin{array}{r}\text { DETECTIONS } \\
\text { NUM }\end{array}$} & \multicolumn{6}{|c|}{ THRESHOLD BASED AUTO WATCHLISTS } & \multicolumn{6}{|c|}{ RANK BASED FORENSIC CASES } \\
\hline & & \multicolumn{2}{|c|}{ FNIR(T), FP(T)=1 } & \multicolumn{2}{|c|}{$\operatorname{FNIR}(\mathrm{T}), \mathrm{FP}(\mathrm{T})=10$} & \multicolumn{2}{|c|}{$\operatorname{FNIR}(\mathrm{T}), \mathrm{FP}(\mathrm{T})=100$} & \multicolumn{2}{|c|}{$\operatorname{FNIR}(\mathrm{R}=1, \mathrm{~T}=0)$} & \multicolumn{2}{|c|}{$\operatorname{FNIR}(\mathrm{R}=5, \mathrm{~T}=0)$} & \multicolumn{2}{|c|}{$\operatorname{FNIR}(\mathrm{R}=20, \mathrm{~T}=0)$} \\
\hline & 1345 & 0.948 & 28 & 0.839 & 29 & 0.698 & 29 & 0.456 & 27 & 0.274 & 26 & 0.173 & 28 \\
\hline A31V & 1345 & 0.879 & 27 & 0.774 & 27 & 0.637 & 27 & 0.448 & 26 & 0.278 & 27 & 0.173 & 27 \\
\hline B30V & 714 & 0.968 & 29 & 0.782 & 28 & 0.641 & 28 & 0.460 & 28 & 0.323 & 30 & 0.222 & 30 \\
\hline $\mathrm{C} 30 \mathrm{~V}$ & 2364 & 0.968 & 31 & 0.907 & 31 & 0.778 & 30 & 0.516 & 31 & 0.395 & 32 & 0.331 & 33 \\
\hline C31V & 2661 & 0.968 & 30 & 0.907 & 30 & 0.782 & 31 & 0.512 & 30 & 0.399 & 33 & 0.331 & 32 \\
\hline D30V & 2004 & 1.000 & 32 & 0.964 & 32 & 0.831 & 32 & 0.560 & 33 & 0.294 & 28 & 0.133 & 24 \\
\hline D31V & 638 & 1.000 & 36 & 0.988 & 33 & 0.855 & 33 & 0.552 & 32 & 0.310 & 29 & 0.210 & 29 \\
\hline E30V & 743 & 0.649 & 24 & 0.419 & 22 & 0.250 & 19 & 0.141 & 20 & 0.093 & 20 & 0.081 & 22 \\
\hline E31V & 743 & 0.484 & 16 & 0.319 & 18 & 0.165 & 17 & 0.093 & 12 & 0.077 & 18 & 0.052 & 19 \\
\hline F30V & 1502 & 1.000 & 35 & 0.996 & 34 & 0.968 & 34 & 0.952 & 34 & 0.895 & 34 & 0.782 & 34 \\
\hline G30V & 493 & 0.577 & 21 & 0.411 & 20 & 0.274 & 21 & 0.206 & 23 & 0.169 & 23 & 0.125 & 23 \\
\hline G31V & 691 & 0.294 & 4 & 0.202 & 8 & 0.097 & 7 & 0.077 & 10 & 0.060 & 16 & 0.052 & 20 \\
\hline G32V & 691 & 0.379 & 8 & 0.194 & 4 & 0.109 & 9 & 0.077 & 8 & 0.056 & 11 & 0.052 & 18 \\
\hline H30V & 668 & 0.327 & 7 & 0.194 & 7 & 0.089 & 6 & 0.077 & 9 & 0.040 & 7 & 0.024 & 6 \\
\hline H31V & 668 & 0.327 & 5 & 0.194 & 5 & 0.089 & 4 & 0.073 & 6 & 0.040 & 4 & 0.024 & 4 \\
\hline $\mathrm{H} 32 \mathrm{~V}$ & 668 & 0.327 & 6 & 0.194 & 6 & 0.089 & 5 & 0.073 & 7 & 0.040 & 6 & 0.024 & 5 \\
\hline I31V & 1382 & 0.472 & 14 & 0.298 & 14 & 0.181 & 18 & 0.117 & 16 & 0.056 & 14 & 0.044 & 14 \\
\hline $\mathrm{J} 30 \mathrm{~V}$ & 441 & 0.403 & 9 & 0.319 & 17 & 0.149 & 16 & 0.121 & 17 & 0.056 & 13 & 0.032 & 8 \\
\hline $\mathrm{J} 31 \mathrm{~V}$ & 441 & 0.407 & 10 & 0.302 & 15 & 0.137 & 12 & 0.125 & 18 & 0.056 & 12 & 0.036 & 11 \\
\hline $\mathrm{J} 32 \mathrm{~V}$ & 441 & 0.435 & 11 & 0.258 & 11 & 0.129 & 11 & 0.105 & 15 & 0.044 & 9 & 0.032 & 10 \\
\hline K30V & 1547 & 0.605 & 22 & 0.480 & 24 & 0.383 & 25 & 0.194 & 22 & 0.113 & 22 & 0.060 & 21 \\
\hline K31V & 941 & 0.452 & 12 & 0.355 & 19 & 0.270 & 20 & 0.149 & 21 & 0.097 & 21 & 0.048 & 17 \\
\hline K32V & 757 & 0.496 & 18 & 0.448 & 23 & 0.323 & 24 & 0.222 & 25 & 0.177 & 25 & 0.145 & 26 \\
\hline K33V & 779 & 0.492 & 17 & 0.419 & 21 & 0.323 & 23 & 0.214 & 24 & 0.169 & 24 & 0.141 & 25 \\
\hline L30V & 526 & 0.839 & 26 & 0.718 & 26 & 0.560 & 26 & 0.484 & 29 & 0.351 & 31 & 0.278 & 31 \\
\hline M30V & 934 & 0.085 & 2 & 0.065 & 3 & 0.032 & 1 & 0.024 & 3 & 0.008 & 3 & 0.008 & 3 \\
\hline M31V & 934 & 0.081 & 1 & 0.065 & 2 & 0.040 & 3 & 0.020 & 2 & 0.008 & 2 & 0.008 & 2 \\
\hline M32V & 934 & 0.089 & 3 & 0.048 & 1 & 0.036 & 2 & 0.016 & 1 & 0.008 & 1 & 0.008 & 1 \\
\hline N30V & 608 & 0.476 & 15 & 0.266 & 12 & 0.141 & 14 & 0.097 & 14 & 0.065 & 17 & 0.044 & 16 \\
\hline N31V & 608 & 0.641 & 23 & 0.302 & 16 & 0.137 & 13 & 0.081 & 11 & 0.052 & 10 & 0.032 & 9 \\
\hline N32V & 608 & 0.452 & 13 & 0.254 & 9 & 0.109 & 10 & 0.060 & 5 & 0.040 & 8 & 0.024 & 7 \\
\hline N33V & 608 & 0.540 & 20 & 0.258 & 10 & 0.105 & 8 & 0.060 & 4 & 0.040 & 5 & 0.040 & 13 \\
\hline Q30V & 501 & 1.000 & 33 & 1.000 & 35 & 0.988 & 36 & 0.972 & 35 & 0.923 & 35 & 0.891 & 35 \\
\hline Q31V & 501 & 1.000 & 34 & 1.000 & 36 & 0.984 & 35 & 0.980 & 36 & 0.956 & 36 & 0.935 & 36 \\
\hline
\end{tabular}

Table 7: For the DATASET U: PASSENGER GATE installation, with 4800 subjects enrolled with a frontal still, the values are identificationmode FNIR(T) for each algorithm at three different decision thresholds corresponding to false positive counts of 1, 10, 100, and investigation-mode FNIR(R) for ranks 1, 5, 20. Each value is accompanied by an integer ranking across all algorithms. The shaded columns indicates the most important metric to watchlist applications. The green shaded cells indicates the most accurate algorithm. Caution: The last column give optimistically low error rates per the arguments of section 4.4.

\begin{tabular}{|c|c|c|c|c|c|c|c|c|c|}
\hline \multicolumn{4}{|c|}{ PARTICIPANT KEY } & SET & SCENE & CAMERA & SET & SCENE & CAMERA \\
\hline $\mathrm{A}=$ DIGITAL BARRIERS & $\mathrm{E}=$ NEUROTECHNOLOGY & $I=$ EYEDEA & $M=N E C$ & C & PHOTOJOURNALISM & PRO & $\mathrm{T}$ & CONCOURSE & PRO \\
\hline $\mathrm{B}=\mathrm{HBINNO}$ & $\mathrm{F}=\mathrm{VAPPLICA}$ & $\mathrm{J}=\mathrm{HISIGN}$ & $\mathrm{N}=$ TOSHIBA & $\mathrm{J}$ & PASSENGER LOADING & PRO & $\mathrm{H}$ & CONCOURSE & PRO \\
\hline $\mathrm{C}=$ VIGILANT & $\mathrm{G}=\mathrm{MORPHO}$ & $\mathrm{K}=$ COGNITEC & $\mathrm{Q}=$ IMAGUS & $\mathrm{P}$ & SPORTS ARENA & CONSUMER & $\mathrm{U}$ & CHOKEPOINT & WEBCAM \\
\hline $\mathrm{D}=\mathrm{AYONIX}$ & $\mathrm{H}=3 \mathrm{M}$ COGENT & $\mathrm{L}=$ CYBEREXTRUDER & $\mathrm{R}=\mathrm{RANK}$ ONE & $\mathrm{L}$ & LUGGAGE RACK & WEBCAM & & & \\
\hline
\end{tabular}




\begin{tabular}{|c|c|c|c|c|c|c|c|c|c|c|c|c|c|}
\hline $\mathrm{N}=48000$ & \multicolumn{2}{|c|}{ NUM ACTORS 248} & \multicolumn{2}{|c|}{ NUM FEEDS 1} & \multicolumn{3}{|c|}{ NUM CLIPS 248} & \multicolumn{3}{|c|}{ NUM FRAMES 11012} & \multicolumn{3}{|c|}{ NUM MINUTES 18.4} \\
\hline & DETECTIONS & \multicolumn{6}{|c|}{ THRESHOLD BASED AUTO WATCHLISTS } & \multicolumn{6}{|c|}{ RANK BASED FORENSIC CASES } \\
\hline ALG & NUM & FNIR(T & $\mathrm{P}(\mathrm{T})=1$ & FNIR( & $=10$ & FNIR(' & 100 & FNIR( & $=0)$ & FNIR( & $=0)$ & FNIR( & $=0)$ \\
\hline A30V & 1345 & 0.992 & 31 & 0.923 & 28 & 0.819 & 28 & 0.718 & 31 & 0.536 & 28 & 0.423 & 28 \\
\hline A31V & 1345 & 0.968 & 28 & 0.927 & 29 & 0.823 & 29 & 0.718 & 30 & 0.528 & 27 & 0.423 & 27 \\
\hline B30V & 714 & 0.968 & 27 & 0.915 & 27 & 0.778 & 27 & 0.645 & 27 & 0.548 & 29 & 0.435 & 30 \\
\hline C30V & 2364 & 0.972 & 30 & 0.956 & 31 & 0.871 & 30 & 0.677 & 29 & 0.577 & 31 & 0.456 & 33 \\
\hline C31V & 2661 & 0.972 & 29 & 0.944 & 30 & 0.883 & 31 & 0.665 & 28 & 0.560 & 30 & 0.456 & 32 \\
\hline D30V & 2004 & 1.000 & 32 & 1.000 & 33 & 0.964 & 32 & 0.786 & 32 & 0.605 & 32 & 0.355 & 26 \\
\hline D31V & 638 & 1.000 & 36 & 1.000 & 36 & 0.988 & 33 & 0.790 & 33 & 0.637 & 33 & 0.440 & 31 \\
\hline E30V & 743 & 0.847 & 24 & 0.569 & 22 & 0.391 & 20 & 0.246 & 18 & 0.149 & 20 & 0.117 & 20 \\
\hline E31V & 743 & 0.770 & 23 & 0.444 & 14 & 0.270 & 13 & 0.153 & 11 & 0.109 & 13 & 0.081 & 16 \\
\hline F30V & 1502 & 1.000 & 35 & 0.996 & 32 & 0.996 & 35 & 0.996 & 36 & 0.980 & 35 & 0.927 & 34 \\
\hline G30V & 493 & 0.714 & 20 & 0.556 & 21 & 0.391 & 19 & 0.310 & 25 & 0.210 & 23 & 0.177 & 23 \\
\hline G31V & 691 & 0.319 & 4 & 0.238 & 5 & 0.149 & 5 & 0.133 & 8 & 0.089 & 10 & 0.065 & 11 \\
\hline G32V & 691 & 0.448 & 5 & 0.238 & 4 & 0.141 & 4 & 0.113 & 4 & 0.073 & 5 & 0.060 & 10 \\
\hline $\mathrm{H} 30 \mathrm{~V}$ & 668 & 0.460 & 9 & 0.294 & 6 & 0.202 & 8 & 0.133 & 7 & 0.077 & 8 & 0.052 & 8 \\
\hline H31V & 668 & 0.460 & 7 & 0.298 & 7 & 0.202 & 6 & 0.137 & 9 & 0.077 & 6 & 0.052 & 6 \\
\hline $\mathrm{H} 32 \mathrm{~V}$ & 668 & 0.460 & 8 & 0.298 & 8 & 0.202 & 7 & 0.137 & 10 & 0.077 & 7 & 0.052 & 7 \\
\hline I30V & 1382 & 0.718 & 21 & 0.480 & 16 & 0.274 & 14 & 0.181 & 14 & 0.109 & 12 & 0.081 & 14 \\
\hline I31V & 1382 & 0.911 & 25 & 0.722 & 25 & 0.444 & 23 & 0.206 & 15 & 0.137 & 16 & 0.089 & 17 \\
\hline $\mathrm{J} 30 \mathrm{~V}$ & 441 & 0.661 & 18 & 0.488 & 19 & 0.327 & 17 & 0.270 & 21 & 0.141 & 19 & 0.081 & 15 \\
\hline J31V & 441 & 0.681 & 19 & 0.476 & 15 & 0.323 & 16 & 0.266 & 20 & 0.141 & 18 & 0.093 & 18 \\
\hline $\mathrm{J} 32 \mathrm{~V}$ & 441 & 0.637 & 17 & 0.431 & 12 & 0.258 & 11 & 0.230 & 16 & 0.121 & 15 & 0.073 & 13 \\
\hline K30V & 1547 & 0.617 & 16 & 0.581 & 23 & 0.484 & 25 & 0.254 & 19 & 0.190 & 22 & 0.145 & 22 \\
\hline K31V & 941 & 0.460 & 6 & 0.444 & 13 & 0.347 & 18 & 0.234 & 17 & 0.141 & 17 & 0.133 & 21 \\
\hline K32V & 757 & 0.560 & 12 & 0.524 & 20 & 0.427 & 22 & 0.294 & 24 & 0.242 & 25 & 0.194 & 25 \\
\hline K33V & 779 & 0.560 & 11 & 0.484 & 17 & 0.411 & 21 & 0.294 & 23 & 0.238 & 24 & 0.194 & 24 \\
\hline L30V & 526 & 0.956 & 26 & 0.839 & 26 & 0.669 & 26 & 0.645 & 26 & 0.516 & 26 & 0.435 & 29 \\
\hline M30V & 934 & 0.250 & 2 & 0.097 & 3 & 0.069 & 3 & 0.044 & 2 & 0.020 & 2 & 0.008 & 2 \\
\hline M31V & 934 & 0.262 & 3 & 0.093 & 2 & 0.065 & 2 & 0.048 & 3 & 0.016 & 1 & 0.008 & 1 \\
\hline M32V & 934 & 0.177 & 1 & 0.085 & 1 & 0.052 & 1 & 0.044 & 1 & 0.024 & 3 & 0.012 & 3 \\
\hline N30V & 608 & 0.573 & 14 & 0.427 & 11 & 0.266 & 12 & 0.161 & 12 & 0.113 & 14 & 0.073 & 12 \\
\hline N31V & 608 & 0.573 & 13 & 0.484 & 18 & 0.274 & 15 & 0.169 & 13 & 0.097 & 11 & 0.056 & 9 \\
\hline N32V & 608 & 0.593 & 15 & 0.371 & 10 & 0.246 & 10 & 0.129 & 6 & 0.077 & 9 & 0.040 & 4 \\
\hline N33V & 608 & 0.516 & 10 & 0.331 & 9 & 0.210 & 9 & 0.117 & 5 & 0.056 & 4 & 0.048 & 5 \\
\hline Q30V & 501 & 1.000 & 33 & 1.000 & 34 & 0.996 & 34 & 0.984 & 34 & 0.976 & 34 & 0.952 & 35 \\
\hline Q31V & 501 & 1.000 & 34 & 1.000 & 35 & 1.000 & 36 & 0.996 & 35 & 0.988 & 36 & 0.980 & 36 \\
\hline R30V & 1472 & 0.758 & 22 & 0.657 & 24 & 0.472 & 24 & 0.278 & 22 & 0.157 & 21 & 0.105 & 19 \\
\hline
\end{tabular}

Table 8: For the DATASET U: PASSENGER GATE installation, with 48000 subjects enrolled with a frontal still, the values are identificationmode FNIR(T) for each algorithm at three different decision thresholds corresponding to false positive counts of 1, 10, 100, and investigation-mode FNIR(R) for ranks 1, 5, 20. Each value is accompanied by an integer ranking across all algorithms. The shaded columns indicates the most important metric to watchlist applications. The green shaded cells indicates the most accurate algorithm. Caution: The last column give optimistically low error rates per the arguments of section 4.4.

\begin{tabular}{|c|c|c|c|c|c|c|c|c|c|}
\hline \multicolumn{4}{|c|}{ PARTICIPANT KEY } & SET & SCENE & CAMERA & SET & SCENE & CAMERA \\
\hline $\mathrm{A}=$ DIGITAL BARRIERS & $\mathrm{E}=$ NEUROTECHNOLOGY & $\mathrm{I}=\mathrm{EYEDEA}$ & $M=N E C$ & C & PHOTOJOURNALISM & PRO & $\mathrm{T}$ & CONCOURSE & PRO \\
\hline $\mathrm{B}=\mathrm{HBINNO}$ & $\mathrm{F}=$ VAPPLICA & $\mathrm{J}=\mathrm{HISIGN}$ & $\mathrm{N}=$ TOSHIBA & $\mathrm{J}$ & PASSENGER LOADING & PRO & $\mathrm{H}$ & CONCOURSE & PRO \\
\hline $\mathrm{C}=$ VIGILANT & $\mathrm{G}=\mathrm{MORPHO}$ & $\mathrm{K}=$ COGNITEC & $\mathrm{Q}=$ IMAGUS & $\mathrm{P}$ & SPORTS ARENA & CONSUMER & U & CHOKEPOINT & WEBCAM \\
\hline $\mathrm{D}=\mathrm{AYONIX}$ & $\mathrm{H}=3 \mathrm{M}$ COGENT & $\mathrm{L}=$ CYBEREXTRUDER & $\mathrm{R}=\mathrm{RANK}$ ONE & $\mathrm{L}$ & LUGGAGE RACK & WEBCAM & & & \\
\hline
\end{tabular}




$$
\mathbf{N}-480-4800-48000
$$
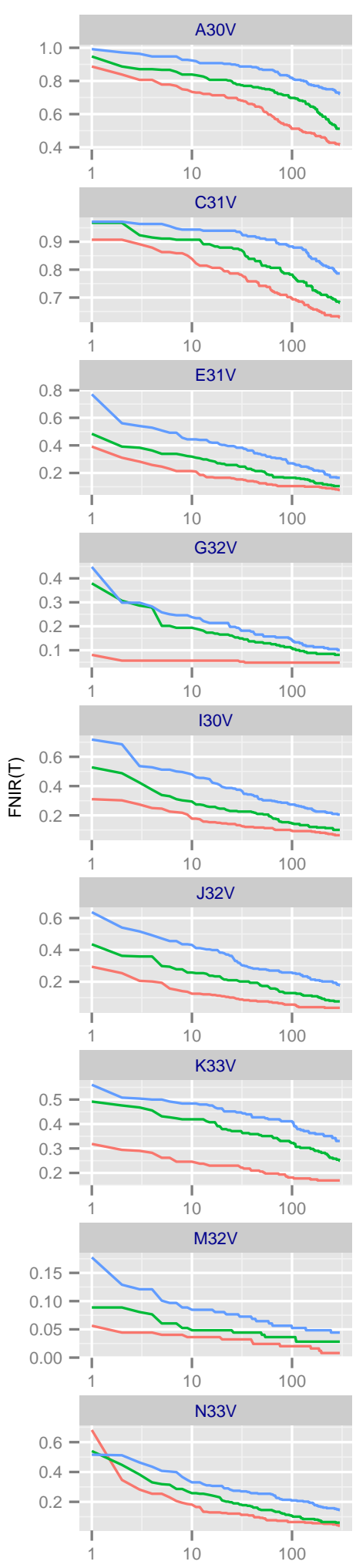

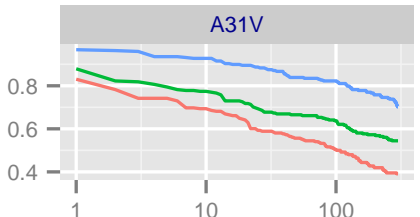

D30V

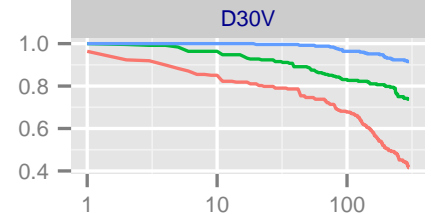

F30V

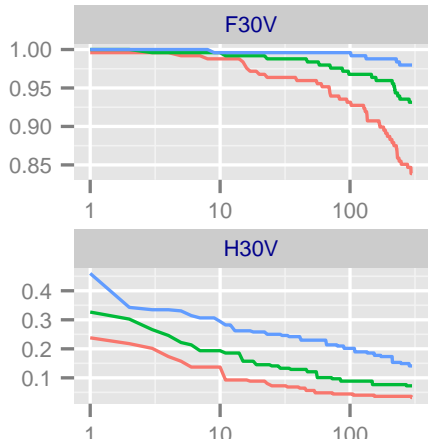

I31V

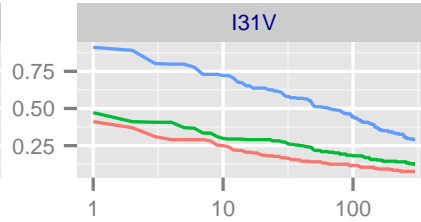

K30V
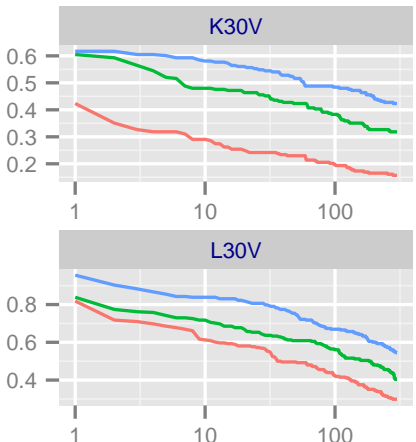

N30V

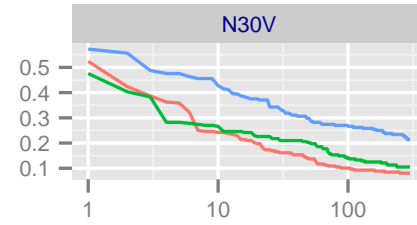

$1.00-\frac{\text { Q30V }}{0.95=-}$

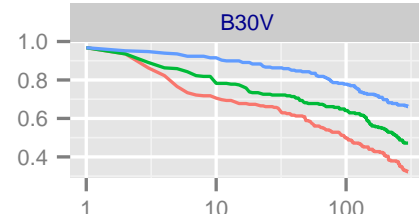

D31V

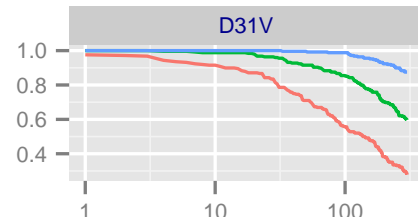

G30V

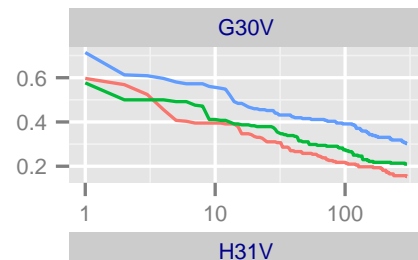

H31V

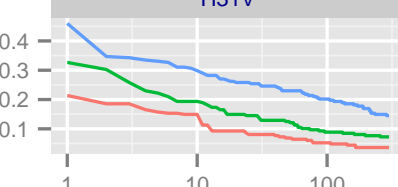

J30V

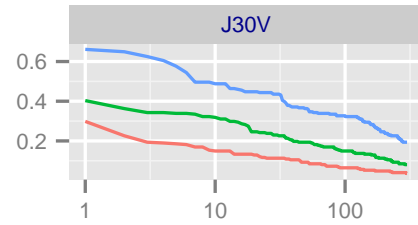

K31V

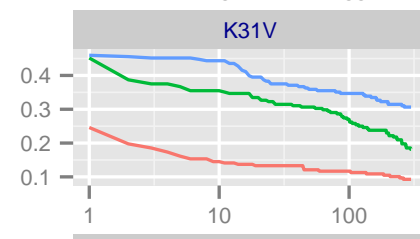

1
$0.25-$
$0.20-$
$0.15-$
$0.10=$
$0.05-$
$0.00-$
1
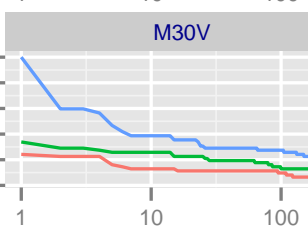

N31V

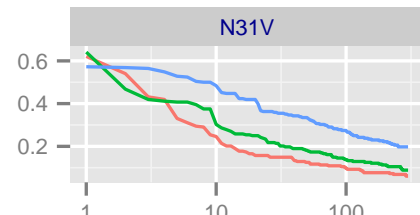

100

Q31V
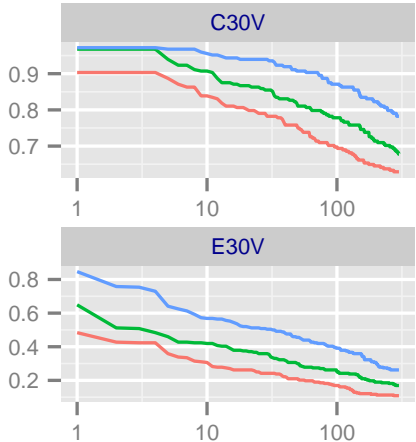

G31V

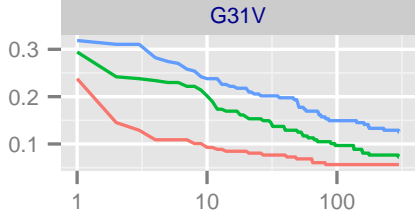

H32V

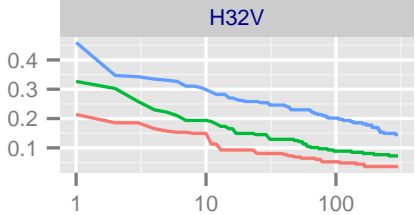

J31V

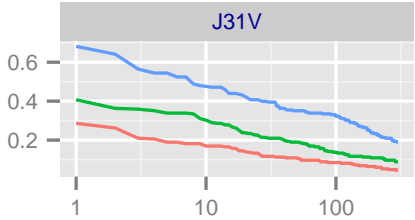

K32V

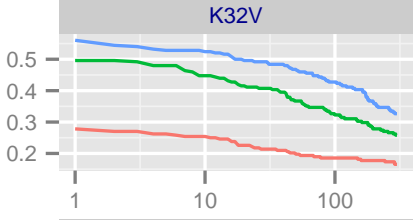

M31V
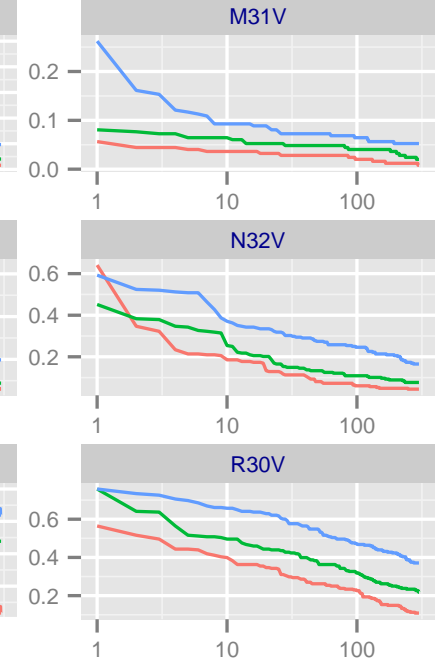

Figure 10: For DATASET U: PASSENGER GATE, the panels show $F N I R(N, L, T)$ vs. $N F P(T)$ for each algorithm at three different gallery sizes. Each trace corresponds to an error tradeoff achieved by sweeping the threshold from low values, at right, to high values, at left. Note the different vertical scales.

\begin{tabular}{|l|l|l|l|}
\hline \multicolumn{4}{|c|}{ PARTICIPANT KEY } \\
\hline A = DIGITAL BARRIERS & E = NEUROTECHNOLOGY & I = EYEDEA & $\mathrm{M}=$ NEC \\
\hline B = HBINNO & F = VAPPLICA & I = HISIGN & N = TOSHIBA \\
\hline C = VIGILANT & G = MORPHO & K = COGNITEC & $\mathrm{Q}=$ IMAGUS \\
\hline D = AYONIX & H = 3M COGENT & L = CYBEREXTRUDER & $\mathrm{R}=$ RANK ONE \\
\hline
\end{tabular}

\begin{tabular}{|l|l|l|l|l|l|}
\hline SET & SCENE & CAMERA & SET & SCENE & CAMERA \\
\hline C & PHOTOJOURNALISM & PRO & T & CONCOURSE & PRO \\
\hline J & PASSENGER LOADING & PRO & H & CONCOURSE & PRO \\
\hline P & SPORTS ARENA & CONSUMER & U & CHOKEPOINT & WEBCAM \\
\hline L & LUGGAGE RACK & WEBCAM & & & \\
\hline
\end{tabular}


Metric $\rightarrow \operatorname{FNIR}(\mathrm{N}, 1,0) \rightarrow \operatorname{FNIR}(\mathrm{N}, \mathrm{L}, \mathrm{T} 10)$
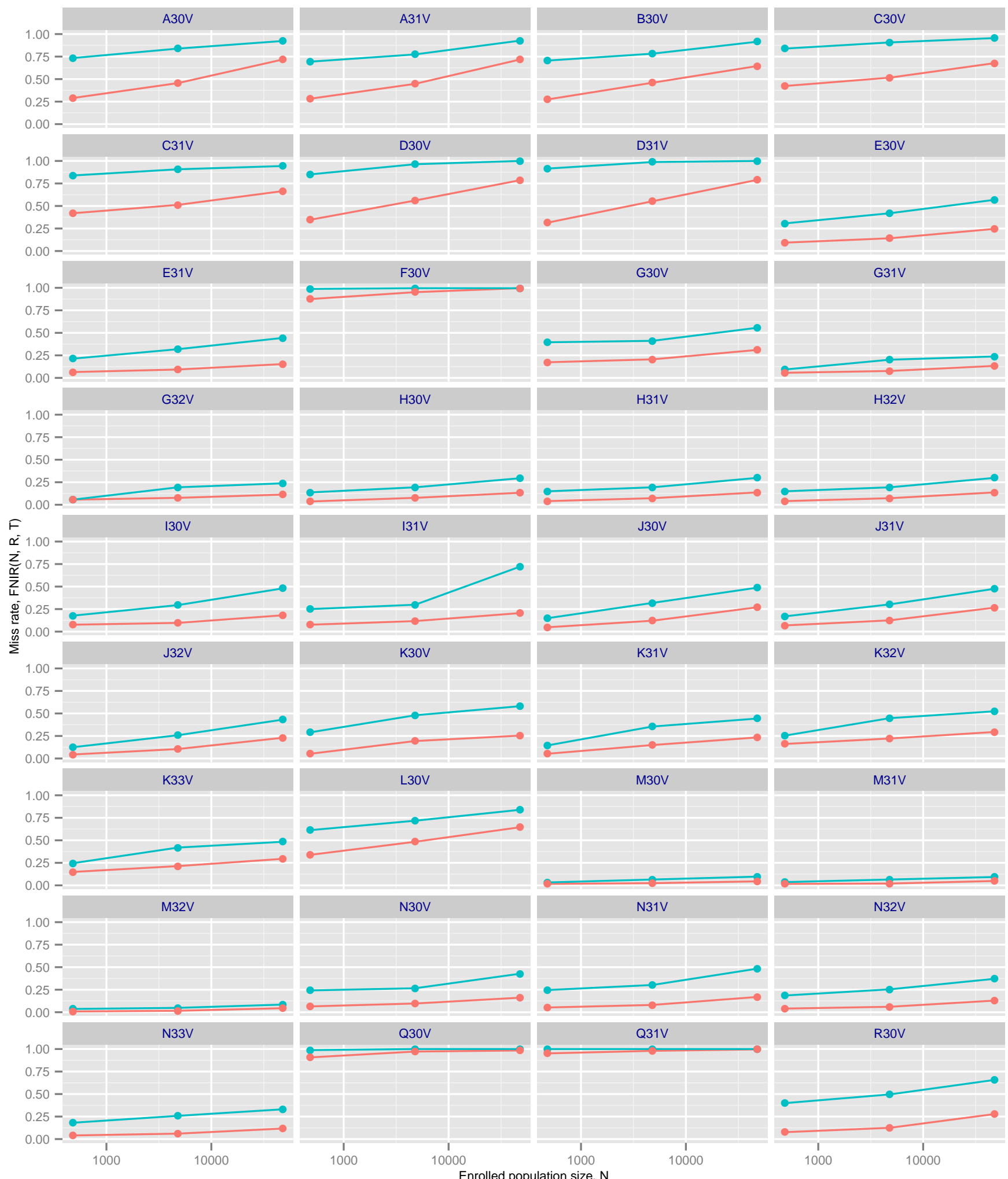

Figure 11: For DATASET U: PASSENGER GATE, the panels show both identification mode high-threshold miss rates, FNIR $(N, L, T)$, and investigation mode zero-threshold miss rates, $\operatorname{FNIR}(N, 1,0)$, as a function of enrolled gallery size, $N$. The threshold is set for each gallery size to elicit ten false positives over all searches for all 248 video clips.

\begin{tabular}{|l|l|l|l|}
\hline \multicolumn{4}{|c|}{ PARTICIPANT KEY } \\
\hline A = DIGITAL BARRIERS & E = NEUROTECHNOLOGY & I = EYEDEA & $\mathrm{M}=$ NEC \\
\hline B = HBINNO & F = VAPPLICA & $\mathrm{J}=$ HISIGN & $\mathrm{N}=$ TOSHIBA \\
\hline C = VIGILANT & G = MORPHO & K = COGNITEC & $\mathrm{Q}=$ IMAGUS \\
\hline D = AYONIX & H = 3M COGENT & L = CYBEREXTRUDER & $\mathrm{R}=$ RANK ONE \\
\hline
\end{tabular}

\begin{tabular}{|l|l|l|l|l|l|}
\hline SET & SCENE & CAMERA & SET & SCENE & CAMERA \\
\hline C & PHOTOJOURNALISM & PRO & T & CONCOURSE & PRO \\
\hline J & PASSENGER LOADING & PRO & H & CONCOURSE & PRO \\
\hline P & SPORTS ARENA & CONSUMER & U & CHOKEPOINT & WEBCAM \\
\hline L & LUGGAGE RACK & WEBCAM & & & \\
\hline
\end{tabular}




\begin{tabular}{|c|c|c|c|c|c|c|c|c|}
\hline DATASET U & \multicolumn{6}{|c|}{ VIDEO SEARCH } & \multirow{2}{*}{\multicolumn{2}{|c|}{$\begin{array}{l}\text { STILL IMAGE } \\
\text { IOD (PIXELS) }\end{array}$}} \\
\hline & NUMBER OF & MEAN TRACK & MEAN TRACK & MEAN & MEAN & MEAN & & \\
\hline ALGORITHM & TRACKS & LENGTH (FRAMES) & EXTENT (FRAMES) & MIN IOD (PX) & MEAN IOD (PX) & MAX IOD (PX) & MEAN & STD. DEV \\
\hline $\mathrm{A} 30 \mathrm{~V}$ & 1345 & 11 & 11 & 45 & 49 & 54 & 116 & 20 \\
\hline A31V & 1345 & 11 & 11 & 45 & 49 & 54 & 116 & 20 \\
\hline B30V & 714 & 23 & 26 & 45 & 58 & 97 & 118 & 21 \\
\hline C30V & 2364 & 4 & 4 & 60 & 62 & 64 & 119 & 21 \\
\hline C31V & 2661 & 4 & 4 & 55 & 57 & 59 & 119 & 21 \\
\hline D30V & 2004 & 5 & 5 & 80 & 82 & 84 & 118 & 22 \\
\hline D31V & 638 & 20 & 20 & 54 & 65 & 75 & 118 & 22 \\
\hline E30V & 743 & 26 & 29 & 38 & 50 & 62 & 117 & 20 \\
\hline E31V & 743 & 26 & 29 & 38 & 50 & 62 & 117 & 20 \\
\hline F30V & 1502 & 5 & 7 & 54 & 62 & 71 & 116 & 20 \\
\hline G30V & 487 & 14 & 20 & 57 & 67 & 76 & 118 & 21 \\
\hline G31V & 662 & 22 & 27 & 42 & 54 & 63 & 117 & 21 \\
\hline G32V & 662 & 22 & 27 & 42 & 54 & 63 & 117 & 21 \\
\hline H30V & 668 & 19 & 21 & 53 & 66 & 77 & 119 & 21 \\
\hline H31V & 668 & 19 & 21 & 53 & 66 & 77 & 119 & 21 \\
\hline $\mathrm{H} 32 \mathrm{~V}$ & 668 & 19 & 21 & 53 & 66 & 77 & 119 & 21 \\
\hline $\mathrm{I} 30 \mathrm{~V}$ & 1382 & 17 & 17 & 30 & 38 & 45 & 120 & 21 \\
\hline I31V & 1382 & 17 & 17 & 30 & 38 & 45 & 120 & 21 \\
\hline $\mathrm{J} 30 \mathrm{~V}$ & 434 & 19 & 23 & 66 & 82 & 95 & 121 & 21 \\
\hline J31V & 434 & 19 & 23 & 66 & 82 & 95 & 121 & 21 \\
\hline $\mathrm{J} 32 \mathrm{~V}$ & 434 & 19 & 23 & 66 & 82 & 95 & 121 & 21 \\
\hline K30V & 1547 & 13 & 16 & 32 & 38 & 43 & 119 & 21 \\
\hline K31V & 941 & 23 & 30 & 31 & 42 & 52 & 119 & 21 \\
\hline K32V & 757 & 17 & 24 & 37 & 44 & 51 & 119 & 21 \\
\hline K33V & 779 & 18 & 25 & 35 & 43 & 49 & 119 & 21 \\
\hline L30V & 526 & 11 & 12 & 50 & 75 & 93 & 117 & 20 \\
\hline M30V & 934 & 19 & 20 & 32 & 40 & 46 & 104 & 19 \\
\hline M31V & 934 & 19 & 20 & 32 & 40 & 46 & 104 & 19 \\
\hline M32V & 934 & 19 & 20 & 32 & 40 & 46 & 104 & 19 \\
\hline N30V & 608 & 24 & 27 & 44 & 58 & 71 & 118 & 21 \\
\hline N31V & 608 & 24 & 27 & 44 & 58 & 71 & 118 & 21 \\
\hline N32V & 608 & 24 & 27 & 44 & 58 & 71 & 118 & 21 \\
\hline N33V & 608 & 24 & 27 & 44 & 58 & 71 & 118 & 21 \\
\hline Q30V & 501 & 12 & 13 & 42 & 48 & 53 & 116 & 20 \\
\hline Q31V & 501 & 12 & 13 & 42 & 48 & 53 & 116 & 20 \\
\hline R30V & 1472 & 1 & 3 & 77 & 78 & 80 & 116 & 20 \\
\hline
\end{tabular}

Table 9: For DATASET U: PASSENGER GATE and each video processing algorithm the table shows: a) the number of reported tracks; b) the mean number of frames reported within those tracks; $c$ ) the mean extent (first minus last frames indices plus one); $d$ ) the mean over all tracks of of the minimum interocular distance (IOD) reported; e) the mean of the mean IOD; $f$ ) the mean of the maximum $I O D ; g)$ the enrollment still image mean IOD; and h) its standard deviation. For some algorithms $(F, J, L, R)$ the tracks don't include all consecutive frames, so the extent of the track can exceed the number of frames in it. For the $D, F$, and $L$ algorithms, the reported still-image eye coordinates are erroneous.

Still portrait IOD: The algorithms have good consensus on the IOD of faces in the enrollment still photographs. The M3xV algorithms, however, report systematically lower IOD values, perhaps because they are not following the ISO/IEC 19794-5 definition of eye centers.

\subsubsection{Computational cost}

In FIVE, algorithms were tasked with detecting and tracking individuals through video clips, and then producing a consolidated template from each detected track. This activity was placed behind a single function call invocation. This placed the responsibility for all image processing, pattern recognition, and feature extraction with the algorithm developer. It absolved the test laboratory (NIST) of making decisions such as about which frames to use, or on how to fuse scores from frame based matching.

Duration: Thus given an input video, the algorithm outputs zero or more templates. Each template has a variable size, which NIST logs. In some cases, zero is the correct response. The size of the templates produced has some operational significance as it can affect network bandwidth and storage requirements, and processing time.

\begin{tabular}{|l|l|l|l|}
\hline \multicolumn{4}{|c|}{ PARTICIPANT KEY } \\
\hline $\mathrm{A}=$ DIGITAL BARRIERS & E = NEUROTECHNOLOGY & $\mathrm{I}=$ EYEDEA & $\mathrm{M}=$ NEC \\
\hline $\mathrm{B}=$ HBINNO & F = VAPPLICA & $\mathrm{J}=$ HISIGN & $\mathrm{N}=$ TOSHIBA \\
\hline $\mathrm{C}=$ VIGILANT & $\mathrm{G}=$ MORPHO & K = COGNITEC & $\mathrm{Q}=$ IMAGUS \\
\hline $\mathrm{D}=$ AYONIX & $\mathrm{H}=$ 3M COGENT & $\mathrm{L}=$ CYBEREXTRUDER & $\mathrm{R}=$ RANK ONE \\
\hline
\end{tabular}

\begin{tabular}{|l|l|l|l|l|l|}
\hline SET & SCENE & CAMERA & SET & SCENE & CAMERA \\
\hline C & PHOTOJOURNALISM & PRO & T & CONCOURSE & PRO \\
\hline J & PASSENGER LOADING & PRO & H & CONCOURSE & PRO \\
\hline P & SPORTS ARENA & CONSUMER & U & CHOKEPOINT & WEBCAM \\
\hline L & LUGGAGE RACK & WEBCAM & & & \\
\hline
\end{tabular}


The plots of Figure 12 show the dependence on the number of input frames, and on the number of faces tracked. The durations are strongly algorithm specific and vary over at least an order of magnitude. In high volume applications like continuous video surveillance, this will have hardware cost implications. The J algorithms operate in fewer than 5 seconds, while the durations for the A algorithms exceeds 50 seconds on average. Each plot includes a text equation which models processing duration as a linear combination of the video length and the number of face tracks found. Its coefficients give the marginal duration increase associated with an additional frame or person. There is often considerable scatter arising, in part, because the algorithms vary in the minimum size of the face that they will detect and, thereby, the length of the track. This is evident in the track statistics of Table 9.

Template size: The size of a template extracted from video imagery is expected to depend on the amount of imagery that was available to algorithm, and on the mechanisms used to extract recognizable features from it. While historically, many researchers have extracted features separately from individual frames, there have long been attempts to integrate information over a track [28]. Indeed, research programs have identified goals to produce representations whose size is independent of the amount of available imagery [12]. This reflects the importance of bounded template size.

Figure 13 shows template size as a function of the length of the track the algorithm reports. The sizes and dependence are highly algorithm-specific. Some algorithms produce fixed template sizes $(\mathrm{G} 3 \mathrm{xV}, \mathrm{J} 3 \mathrm{xV}, \mathrm{K} 32 \mathrm{~V}, \mathrm{Q} 3 \mathrm{xV})$. The implementations may be selecting a best-frame from the video, or integrating information temporally - in a black-box test, we do not know. Other algorithms tend to produce larger templates with a dependence on track length. Algorithms A3xV, B30V, D3xV, and R30V have a nearly perfect linear relationship. Other algorithms - K31V, K33V, M3xV - give the linear relationship but impose a hard limit on the template size above which additional features are not added even though the track is longer.

\begin{tabular}{|l|l|l|l|}
\hline \multicolumn{5}{|c|}{ PARTICIPANT KEY } \\
\hline $\mathrm{A}=$ DIGITAL BARRIERS & E = NEUROTECHNOLOGY & I = EYEDEA & $\mathrm{M}=$ NEC \\
\hline $\mathrm{B}=$ HBINNO & $\mathrm{F}=$ VAPPLICA & $\mathrm{J}=$ HISIGN & $\mathrm{N}=$ TOSHIBA \\
\hline $\mathrm{C}=$ VIGILANT & $\mathrm{G}=$ MORPHO & $\mathrm{K}=$ COGNITEC & $\mathrm{Q}=$ IMAGUS \\
\hline $\mathrm{D}=$ AYONIX & $\mathrm{H}=3 \mathrm{M}$ COGENT & $\mathrm{L}=$ CYBEREXTRUDER & $\mathrm{R}=$ RANK ONE \\
\hline
\end{tabular}

\begin{tabular}{|l|l|l|l|l|l|}
\hline SET & SCENE & CAMERA & SET & SCENE & CAMERA \\
\hline C & PHOTOJOURNALISM & PRO & T & CONCOURSE & PRO \\
\hline J & PASSENGER LOADING & PRO & H & CONCOURSE & PRO \\
\hline P & SPORTS ARENA & CONSUMER & U & CHOKEPOINT & WEBCAM \\
\hline L & LUGGAGE RACK & WEBCAM & & & \\
\hline
\end{tabular}


numk $\rightarrow \mathrm{K}=0 \rightarrow \mathrm{K}=1-\mathrm{K}=2-\mathrm{K}=3 \square \mathrm{K}=4 \square \mathrm{K}>=4$
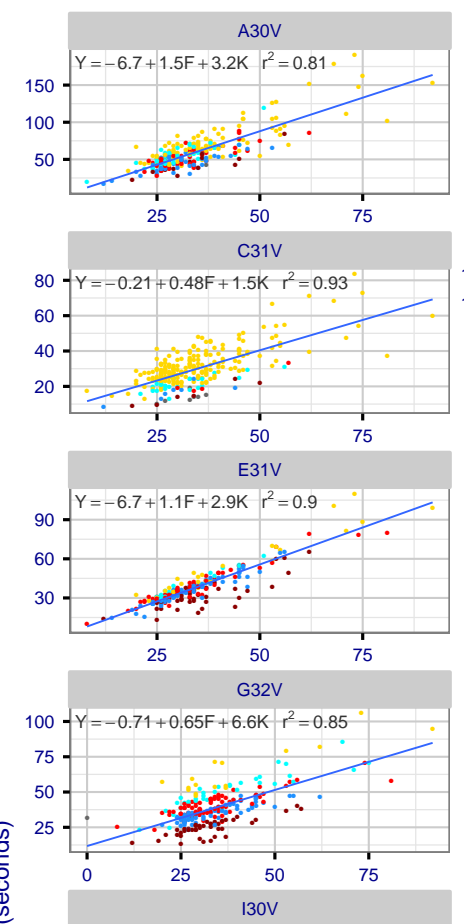

() $20=-1.2+0.096 \mathrm{~F}+0.69 \mathrm{~K} \quad r^{2}=0.73$

E 20

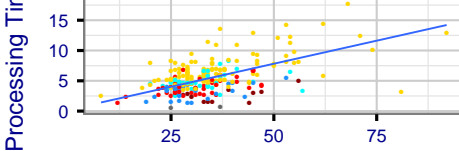

造 $10.0-\mathrm{J} 32 \mathrm{~V}$

$\stackrel{0}{>} 10.0-\mathrm{Y}=-0.12+0.076 \mathrm{~F}+0.38 \mathrm{~K} \quad \mathrm{r}^{2}=0.46$

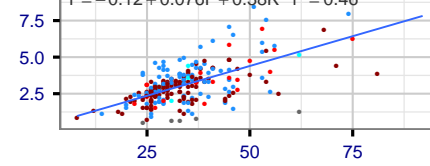
$\mathrm{K} 33 \mathrm{~V}$

$80-Y=-5.8+0.65 F+3.3 K \quad r^{2}=0.88$

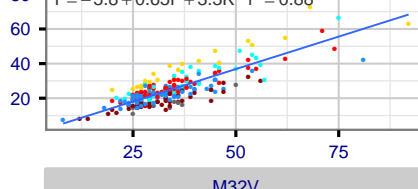

$332 \mathrm{~V}$

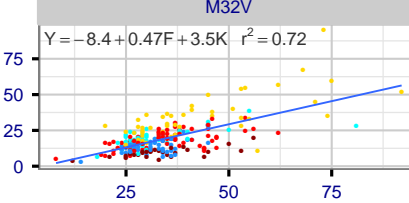

N33V

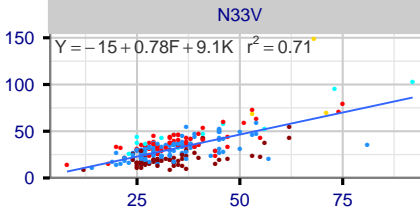

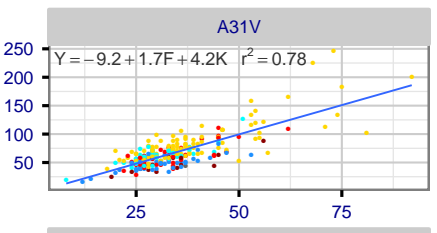

D $30 \mathrm{~V}$
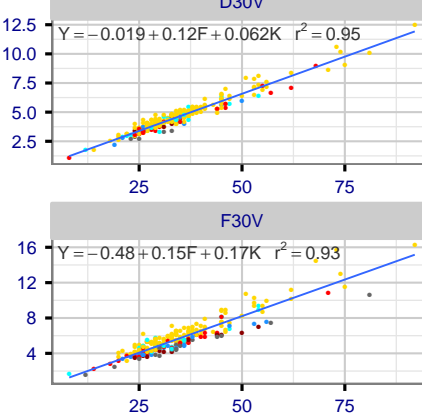

$\mathrm{H} 30 \mathrm{~V}$

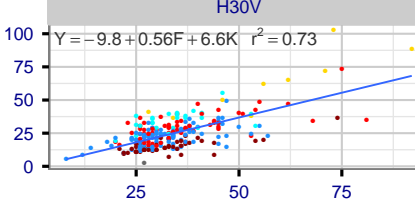

$131 \mathrm{~V}$

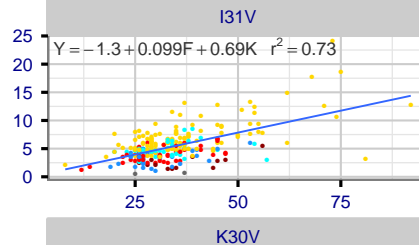

$\mathrm{K} 30 \mathrm{~V}$

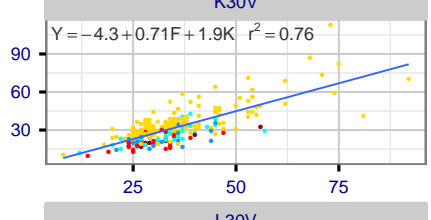

L30V
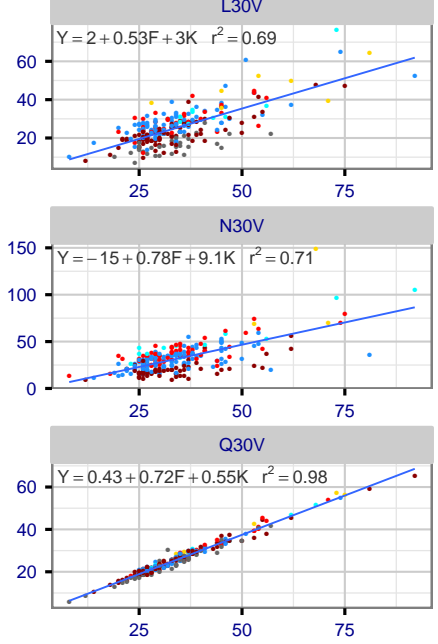

Number of Frames in Video Clip
B30V

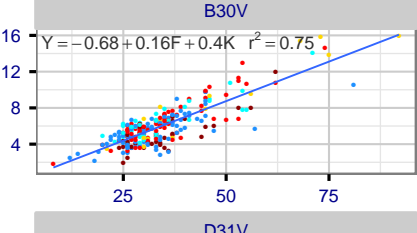

$20-Y=-0.21+0.21 F+0.3 K \quad r^{2}=0.96$

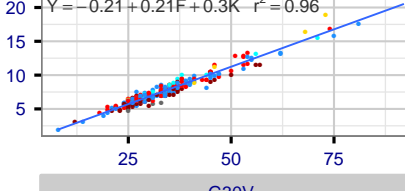

G30V
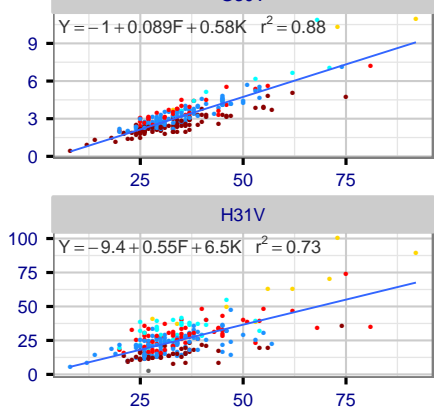

J30V
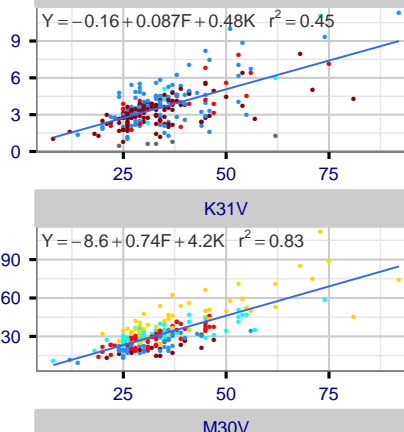

$100-\mathrm{M} 30 \mathrm{~V}$

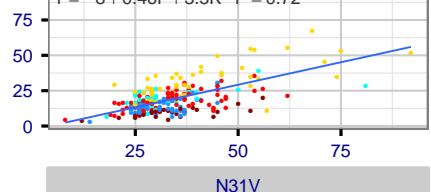

$150-\mathrm{N}=-15+0.78 \mathrm{~F}+9.1 \mathrm{~K} \quad \mathrm{r}^{2}=0.71$
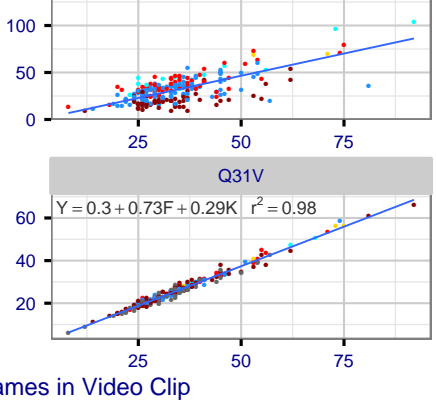
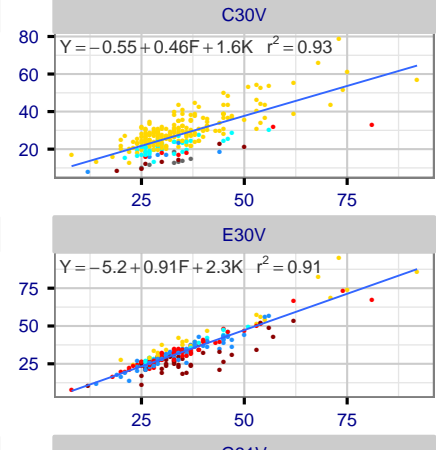

G31V

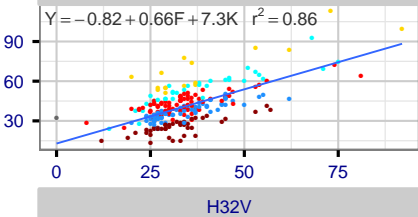

$100-\mathrm{Y}=-9.7+0.55 \mathrm{~F}+6.6 \mathrm{~K} \mathrm{r}^{2}=0.73$

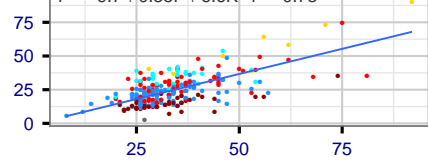

J31V


$20-\mathrm{Y}=-0.82+0.22 \mathrm{~F}+0.33 \mathrm{~K} \mathrm{r}^{2}=0.8$

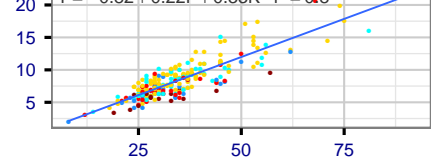

Figure 12: For DATASET U: PASSENGER GATE, the plots show the duration of the video processing as a function of the number of frames passed to the algorithm. The duration varies also with the number of face tracks found in the clip, $K$, which is color coded. The regression formula is one of several simple models. Its coefficients can be interpreted as the marginal cost of adding one additional frame, or face, to the video.

\begin{tabular}{|c|c|c|c|c|c|c|c|c|c|}
\hline \multicolumn{4}{|c|}{ PARTICIPANT KEY } & \multirow{2}{*}{\begin{tabular}{|l|} 
SET \\
$\mathrm{C}$ \\
\end{tabular}} & SCENE & \multirow{2}{*}{\begin{tabular}{|l|} 
CAMERA \\
PRO
\end{tabular}} & \multirow{2}{*}{\begin{tabular}{|l|} 
SET \\
$\mathrm{T}$ \\
\end{tabular}} & \multirow{2}{*}{\begin{tabular}{|l|} 
SCENE \\
CONCOURSE \\
\end{tabular}} & \multirow{2}{*}{\begin{tabular}{|l} 
CAMERA \\
PRO
\end{tabular}} \\
\hline $\mathrm{A}=$ DIGITAL BARRIERS & $\mathrm{E}=$ NEUROTECHNOLOGY & $\mathrm{I}$ = EYEDEA & $M=N E C$ & & PHOTOJOURNALISM & & & & \\
\hline $\mathrm{C}=$ VIGILANT & $\mathrm{G}=\mathrm{MORPHO}$ & $\mathrm{K}=$ COGNITEC & $Q=$ IMAGUS & $\mathrm{P}$ & SPORTS ARENA & CONSUMER & $\mathrm{U}$ & CHOKEPOINT & WEBCAM \\
\hline $\mathrm{D}=$ AYONIX & $\mathrm{H}=3 \mathrm{M}$ COGENT & $\mathrm{L}=$ CYBEREXTRUDER & $\mathrm{R}=\mathrm{RANK}$ ONE & $\mathrm{L}$ & LUGGAGE RACK & WEBCAM & & & \\
\hline
\end{tabular}



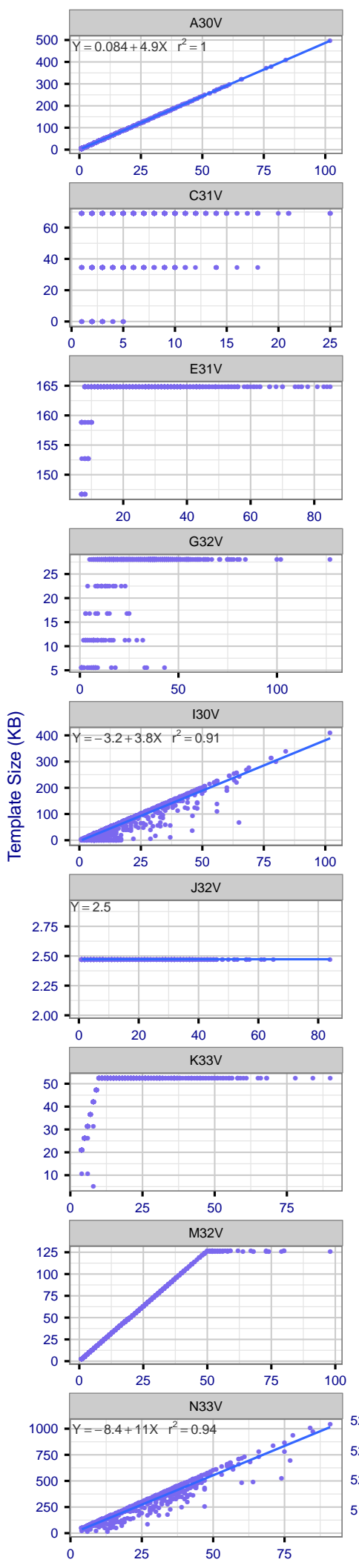
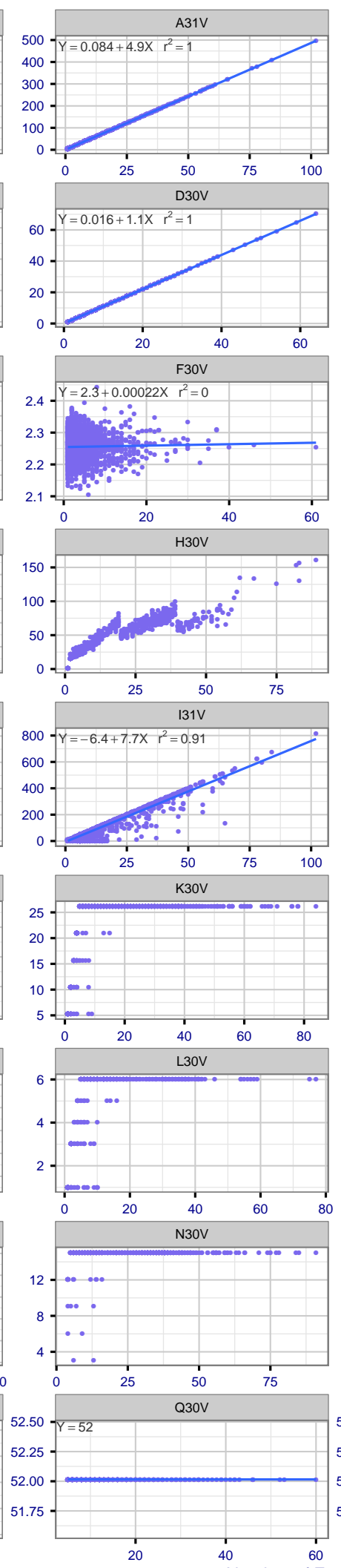

Number of Frames in Face Track
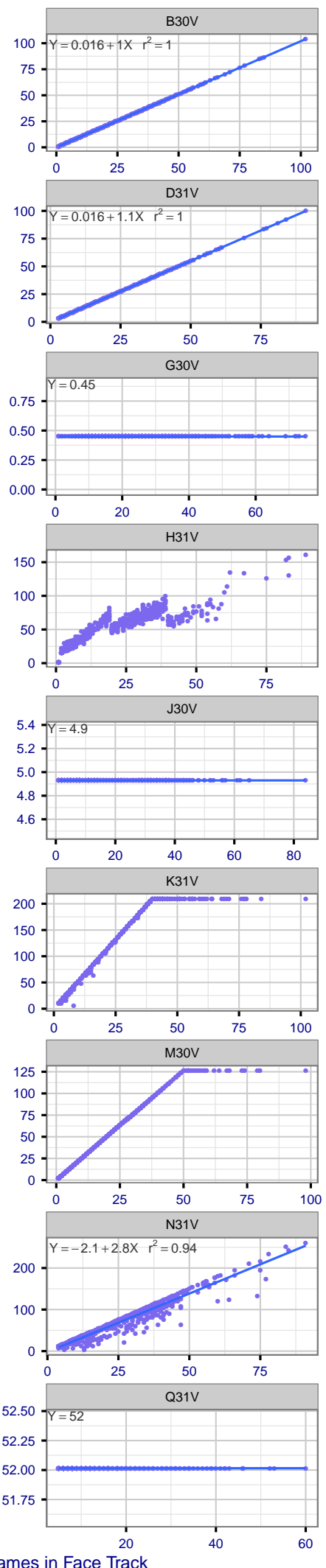
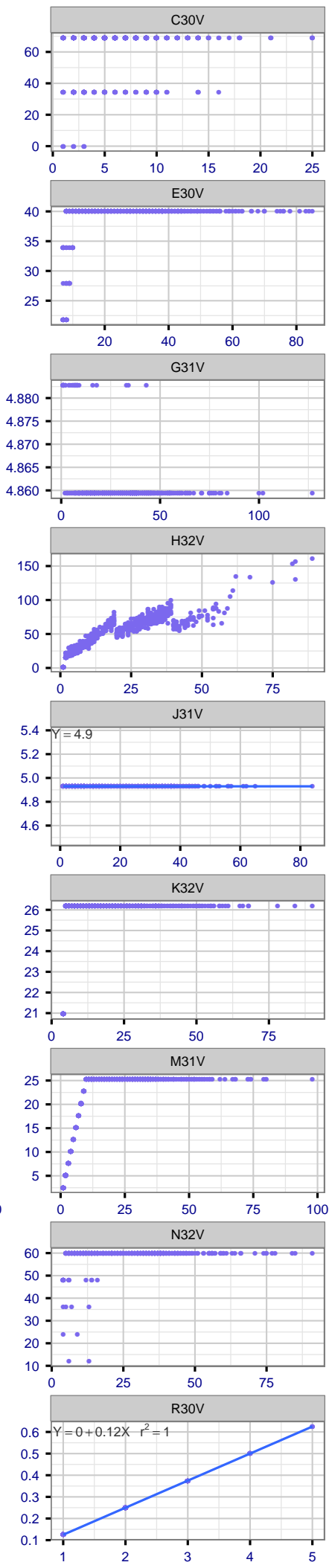

Figure 13: For DATASET U: PASSENGER GATE, the plots show the template size for features extracted from video face tracks as a function of the length of the track reported by the algorithm, in frames. Note the R30V algorithm reports very few frames, and these are irregularly spaced throughout the clip. A best fit linear model is also plotted, when appropriate.

\begin{tabular}{|l|l|l|l|}
\hline \multicolumn{4}{|c|}{ PARTICIPANT KEY } \\
\hline A = DIGITAL BARRIERS & E = NEUROTECHNOLOGY & I = EYEDEA & M = NEC \\
\hline B = HBINNO & F = VAPPLICA & J = HISIGN & N = TOSHIBA \\
\hline C = VIGILANT & G = MORPHO & K = COGNITEC & Q = IMAGUS \\
\hline D = AYONIX & H = 3M COGENT & L = CYBEREXTRUDER & R = RANK ONE \\
\hline
\end{tabular}

\begin{tabular}{|l|l|l|l|l|l|}
\hline SET & SCENE & CAMERA & SET & SCENE & CAMERA \\
\hline C & PHOTOJOURNALISM & PRO & T & CONCOURSE & PRO \\
\hline J & PASSENGER LOADING & PRO & H & CONCOURSE & PRO \\
\hline P & SPORTS ARENA & CONSUMER & U & CHOKEPOINT & WEBCAM \\
\hline L & LUGGAGE RACK & WEBCAM & & & \\
\hline
\end{tabular}



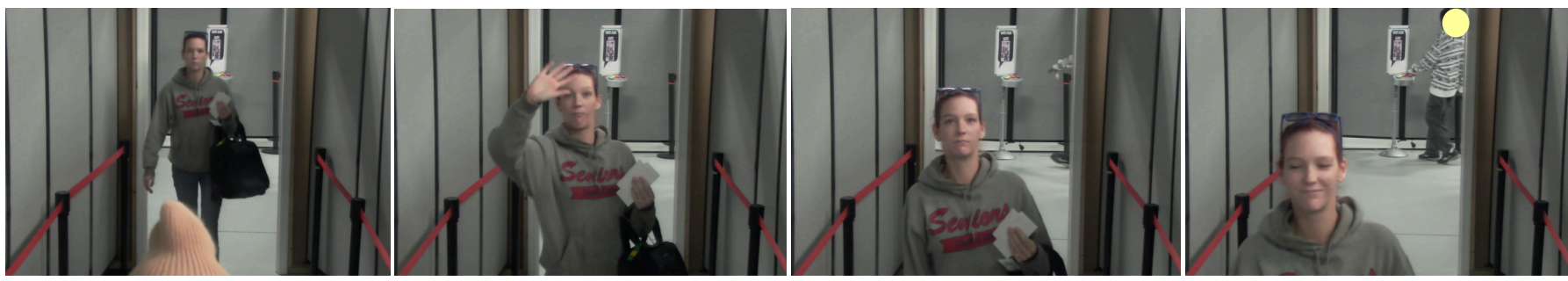

Figure 14: Example images from the ceiling mounted camera for the free movement scenarios from the DATASET J: PASSENGER LOADING BRIDGE dataset. **The images in this table are from the subject S1115 in the DHS / S\&T provided AEER dataset. The subject gave written opt-in permission to allow public release of all imagery. Where consent from individuals in the background was not obtained, their faces were masked (yellow circle).

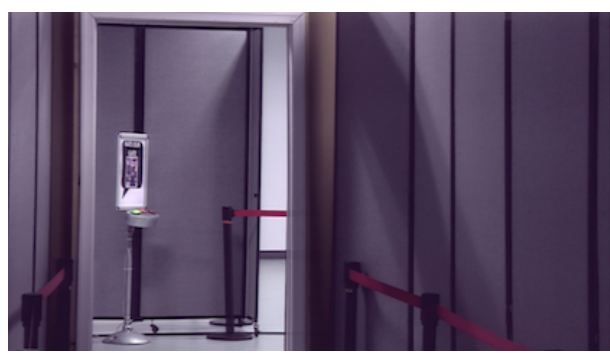

(a) Right Wall Mounted

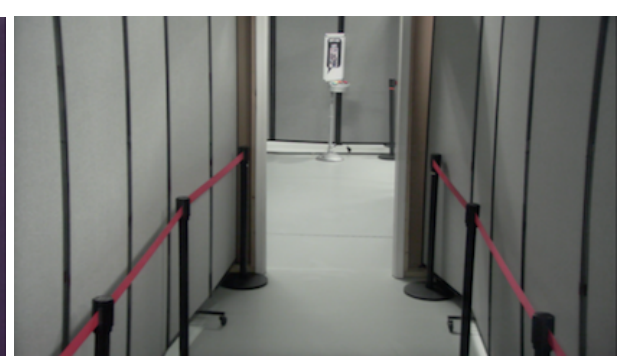

(b) Ceiling Mounted

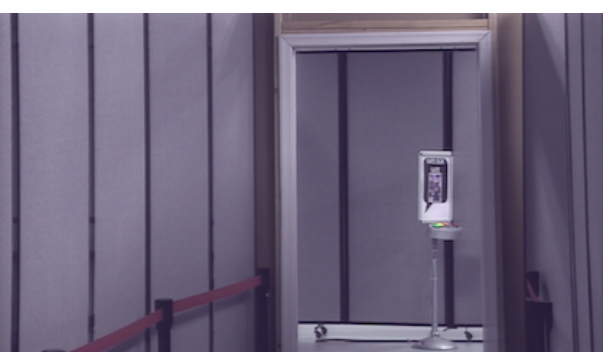

(c) Left Wall Mounted

Figure 15: Fields of view for the three cameras used in DATASET J: PASSENGER LOADING BRIDGE . ${ }^{* *}$ The images in this table are from the DHS / S\&T provided AEER dataset.

\subsection{DATASET J: PASSENGER LOADING BRIDGE}

Overview: This dataset consists of videos of subjects walking along a purpose-built simulated passenger loading bridge (PLB). The PLB was equipped with three cameras, one ceiling mounted, with two more mounted on the walls symmetrically below it, in a vertical plane. All cameras were angled to observe subjects walking toward them - see Figure 14 - their orientation was selected to be favorable for face recognition with respect to the angle of the optical axis and the face normal - see Figure 15. Key imaging properties for this dataset are summarized in Table 10.

\begin{tabular}{|l|l|}
\hline Property & Value \\
\hline Camera & Vaddio PowerView (PTZ) \\
\hline Camera mounting & Attached to display observed by subject \\
\hline Camera height & 2.44 meters (ceiling); and 1.83 meters (wall mounted cameras) \\
\hline Camera orientation & Both elevation from ceiling and azimuth to sidewall below $15^{\circ}$ \\
\hline Range to subject & $1 \mathrm{~m}-5 \mathrm{~m}$ \\
\hline Frame rate & $30 \mathrm{sec}^{-1}$ \\
\hline Width & 1920 \\
\hline Height & 1080 \\
\hline Chroma sampling & YUV420 \\
\hline Nominal bitrate & \\
\hline Codec & AVC H264 \\
\hline
\end{tabular}

Table 10: Key imaging properties for DATASET J: PASSENGER LOADING BRIDGE

Experimental Design: The video clips show volunteer recruits acting as passengers walking along a PLB simulating an aircraft boarding process. There are 48 clips in total, with 16 from each of the three cameras. The 16 videos represent the appearance of 8 groups of people on two occasions, about 30 minutes apart. Each group has between 42 and 51 volunteer subjects. The groups were exposed to different experimental manipulations. To examine the effect of walking on recognition accuracy, an articial bottleneck was applied at the exit of the PLB. The result is that four groups of people walked freely past the cameras without stopping, and another four groups were "bottlenecked" mostly standing in a

\begin{tabular}{|l|l|l|l|}
\hline \multicolumn{4}{|c|}{ PARTICIPANT KEY } \\
\hline $\mathrm{A}=$ DIGITAL BARRIERS & E = NEUROTECHNOLOGY & I = EYEDEA & $\mathrm{M}=$ NEC \\
\hline $\mathrm{B}=$ HBINNO & $\mathrm{F}=$ VAPPLICA & $\mathrm{J}=$ HISIGN & $\mathrm{N}=$ TOSHIBA \\
\hline $\mathrm{C}=$ VIGILANT & $\mathrm{G}=$ MORPHO & $\mathrm{K}=$ COGNITEC & $\mathrm{Q}=$ IMAGUS \\
\hline $\mathrm{D}=$ AYONIX & $\mathrm{H}=$ 3M COGENT & L = CYBEREXTRUDER & $\mathrm{R}=$ RANK ONE \\
\hline
\end{tabular}

\begin{tabular}{|l|l|l|l|l|l|}
\hline SET & SCENE & CAMERA & SET & SCENE & CAMERA \\
\hline C & PHOTOJOURNALISM & PRO & T & CONCOURSE & PRO \\
\hline J & PASSENGER LOADING & PRO & H & CONCOURSE & PRO \\
\hline P & SPORTS ARENA & CONSUMER & U & CHOKEPOINT & WEBCAM \\
\hline L & LUGGAGE RACK & WEBCAM & & & \\
\hline
\end{tabular}




\begin{tabular}{|l|l|}
\hline Quantity & Value or description \\
\hline Mode & Video search to still enrollment \\
\hline Number of actors & 354, with subsets in different experiments \\
\hline Number of non-actors & 0 \\
\hline Number of cameras & 3 , one ceiling, two wall mounted, symmetrically \\
\hline Video duration with actors & 485 minutes \\
\hline Video duration no actors & 0 \\
\hline Subject motion & Usually single file toward and below the camera. Walking or stopped in queue. \\
\hline Number of clips & $48=2$ (walk, queue) x 3 cameras $x$ 2 attractor content $x$ 2 repeats $x$ 2 attractor on/off \\
\hline Clip duration & Varies from min 7mins 15 seconds to 12 mins 48 seconds, mean 10.1 minutes \\
\hline Number of enrolled subjects & 480 \\
\hline Number of enrolled stills & 1 per subject \\
\hline Properties of enrolled stills & Frontal, close ICAO compliance; Mean IOD 106 pixels \\
\hline FNIR estimation & Actors present video vs. enrolled gallery \\
\hline FPIR estimation & Actors present video vs. separate non-actor gallery \\
\hline Candidate list length & 20 \\
\hline Number of persons in FOV & {$[0,3]$ free movement or [4,7] queued, approx. } \\
\hline Video ground truth & Style A: See Figure 6 \\
\hline
\end{tabular}

Table 11: Key experimental design the DATASET J: PASSENGER LOADING BRIDGE results.

queue and occasionally moving forward. This factor represents the situation that often occurs when boarding twin-aisle vs. single-aisle aircraft. People in the latter scenario are typically in the camera's field of view for a longer period, but their behavior is less consistent - see Figure 14 - subjects would often look down or away from the camera. In addition, some faces are temporarily occluded by the people standing in front of them.

Each of the groups was also exposed, or not, to a video display attractor installed just above the ceiling-mounted camera. For four groups the attractor was off. For four others, the attractor was switched on. Two of those saw a "live agent" which was a rotating sequence of around six different people greeting the subjects and included both audio and video. The other two groups saw a "digital mirror" from the ceiling mounted camera showing their own live video fed back to them. This encouraged subjects to misbehave by exagerrating expressions - see Figure 14 - something not observed for the "live agent" content.

Mated scores are computed over long video clips from each of three cameras. The video clips of freely walking subjects last about from 8 to 10 minutes. The video clips of queued subjects last from 10 to 12 minutes. The clips were passed to the algorithms in their entirety. These are searched against a gallery of $N=480$ images, one from each of the 354 actors, and an additional 126 from a disjoint background population. Enrolled still images: Enrollment images were collected cooperatively using a consumer-grade SLR - see Figure 16. These are in good conformance to the ISO/IEC 19794-5 full frontal image type, aside from some additional torso and background. Exactly one image is enrolled per subject.

Nonmated scores are computed by comparing templates generated from video clips against the global nonmated enrollment dataset. Thresholds are generally computed on a per-camera basis, i.e. using scores only from that camera's searches.

Key experimental design details are summarized in Table 11.

\begin{tabular}{|l|l|l|l|}
\hline \multicolumn{5}{|c|}{ PARTICIPANT KEY } \\
\hline $\mathrm{A}=$ DIGITAL BARRIERS & $\mathrm{E}=$ NEUROTECHNOLOGY & $\mathrm{I}=$ EYEDEA & $\mathrm{M}=$ NEC \\
\hline $\mathrm{B}=$ HBINNO & $\mathrm{F}=$ VAPPLICA & $\mathrm{J}=$ HISIGN & $\mathrm{N}=$ TOSHIBA \\
\hline $\mathrm{C}=$ VIGILANT & $\mathrm{G}=$ MORPHO & $\mathrm{K}=$ COGNITEC & $\mathrm{Q}=$ IMAGUS \\
\hline $\mathrm{D}=$ AYONIX & $\mathrm{H}=$ 3M COGENT & $\mathrm{L}=$ CYBEREXTRUDER & $\mathrm{R}=$ RANK ONE \\
\hline
\end{tabular}

\begin{tabular}{|l|l|l|l|l|l|}
\hline SET & SCENE & CAMERA & SET & SCENE & CAMERA \\
\hline C & PHOTOJOURNALISM & PRO & T & CONCOURSE & PRO \\
\hline J & PASSENGER LOADING & PRO & H & CONCOURSE & PRO \\
\hline P & SPORTS ARENA & CONSUMER & U & CHOKEPOINT & WEBCAM \\
\hline L & LUGGAGE RACK & WEBCAM & & & \\
\hline
\end{tabular}




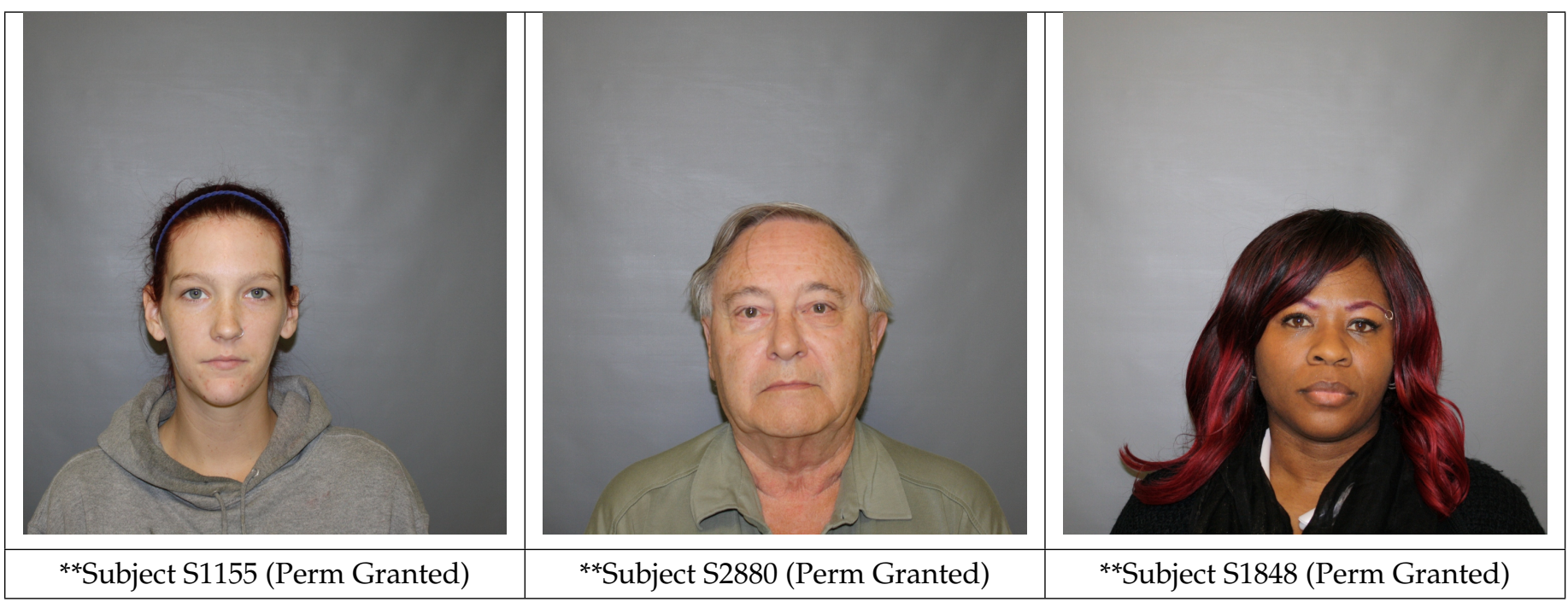

Figure 16: DATASET J: PASSENGER LOADING BRIDGE . Examples of enrollment images collected with Canon SLR camera. ${ }^{* *}$ The face images in this figure are from the DHS / S\&T provided AEER dataset. The included subjects consented to release their images in public reports.

Results: Figure 17 shows $\operatorname{FNIR}(N, L, T)$ identification-mode miss rates in bar form for convenient visual comparison. Similarly, Figure 18 shows FNIR $(N, 1,0)$ investigation-mode miss rates. The decision threshold (Fig. 17) is set to elicit 10 false positives over all 354 subjects appearing in all clips from the respective camera (so both with and without the attractor enabled). While, this approximately corresponds to fewer than one false positive for every thirty four subjects walking down the passenger loading bridge, each subject walked along the PLB twice, and each algorithm might detect and search several templates from each person - see Figure 3.

Notable observations are:

Attractors are effective: Considering just the ceiling mounted camera, alongside which the TV display attractor is mounted, the lowest error rates are observed when the attractor as switched on, and when the subjects were waiting in line.

Ceiling mounted camera is superior: The overhead camera gives the best accuracy, outperforming the cameras mounted on the side walls of the passenger loading bridge. This comparison must be made with the attractor off (the first two columns of Figure 17) because the side-mounted cameras were not equipped with an attractor. For the five most accurate algorithm developers $(\mathrm{M}, \mathrm{H}, \mathrm{J}, \mathrm{I}, \mathrm{N})$, the identification miss rates for the ceiling mounted camera are as much as half of those for the side mounted cameras. However this effect applies to subjects detained in a queue. It is much reduced for freely walking subjects. This latter result supports the assertion that elevation angle is important to face recognition, of a similar magnitude to the yaw angles inherent in the use of the wall-mounted cameras. The G30V algorithm gives better accuracy with wall-mounted cameras than with ceiling mounted when the attractor is disabled.

Uncertainty in error rates: While the lowest $\operatorname{FNIR}(T)$ value for a single camera is below $4 \%$ (algorithm M32V, ceiling camera, attractor enabled, queued), there is considerable uncertainty associated with this measurement, stemming from the small sample size - here just 88 subjects. This small population size imparts some uncertainty

\begin{tabular}{|l|l|l|l|}
\hline \multicolumn{5}{|c|}{ PARTICIPANT KEY } \\
\hline $\mathrm{A}=$ DIGITAL BARRIERS & $\mathrm{E}=$ NEUROTECHNOLOGY & $\mathrm{I}=$ EYEDEA & $\mathrm{M}=$ NEC \\
\hline $\mathrm{B}=$ HBINNO & $\mathrm{F}=$ VAPPLICA & $\mathrm{J}=$ HISIGN & $\mathrm{N}=$ TOSHIBA \\
\hline $\mathrm{C}=$ VIGILANT & $\mathrm{G}=$ MORPHO & $\mathrm{K}=$ COGNITEC & $\mathrm{Q}=$ IMAGUS \\
\hline $\mathrm{D}=$ AYONIX & $\mathrm{H}=$ 3M COGENT & $\mathrm{L}=$ CYBEREXTRUDER & $\mathrm{R}=$ RANK ONE \\
\hline
\end{tabular}

\begin{tabular}{|l|l|l|l|l|l|}
\hline SET & SCENE & CAMERA & SET & SCENE & CAMERA \\
\hline C & PHOTOJOURNALISM & PRO & T & CONCOURSE & PRO \\
\hline J & PASSENGER LOADING & PRO & H & CONCOURSE & PRO \\
\hline P & SPORTS ARENA & CONSUMER & U & CHOKEPOINT & WEBCAM \\
\hline L & LUGGAGE RACK & WEBCAM & & & \\
\hline
\end{tabular}




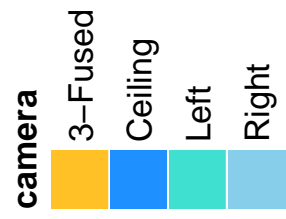
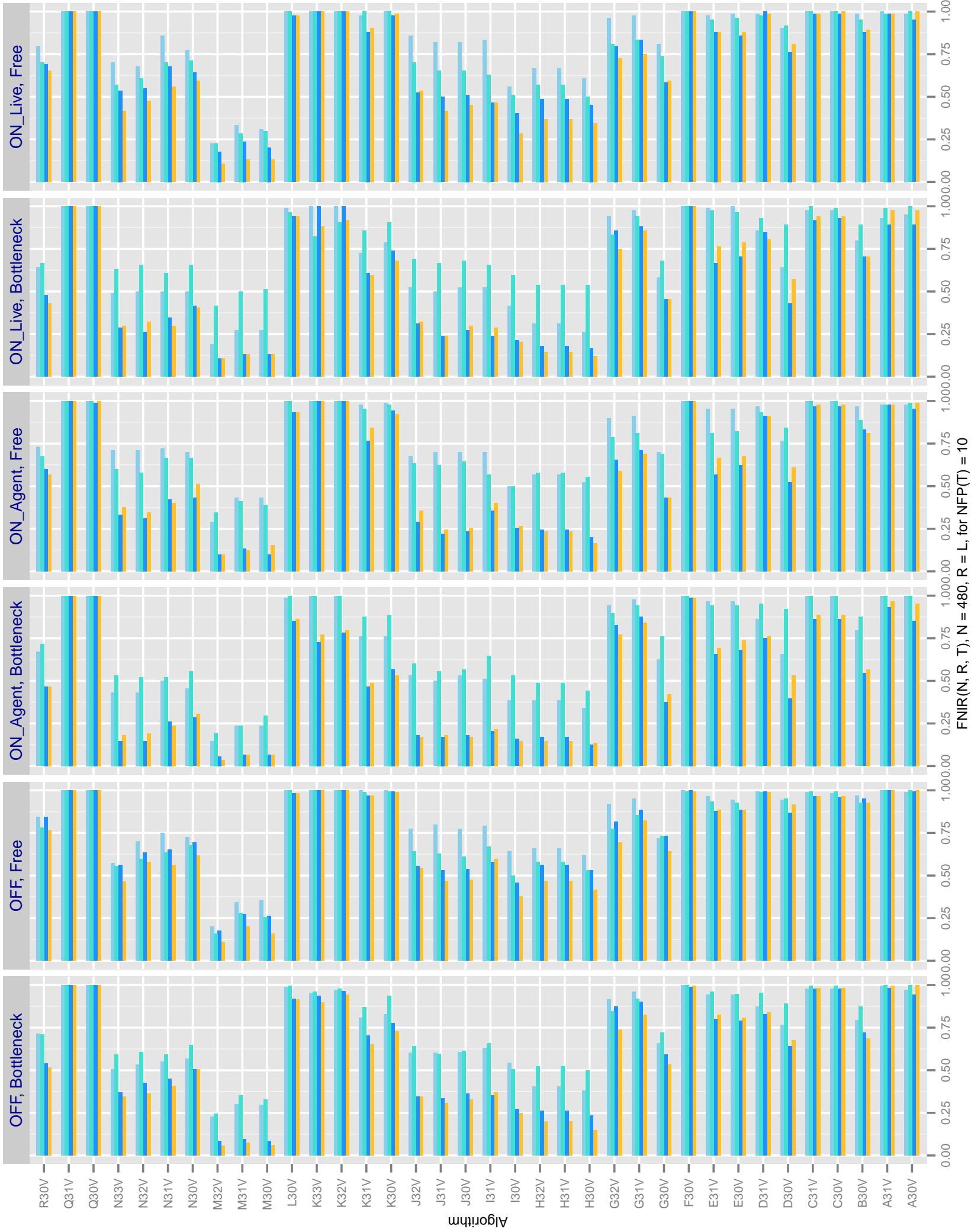

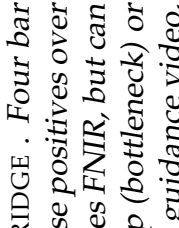

总通弯

원ㅎㅀ

$111 \overline{8}$

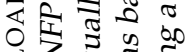

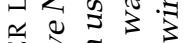

당

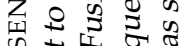

के

$\ddot{H}$ 元

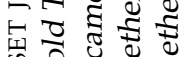

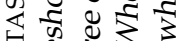

๘ี

पे

距

ฮี่

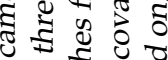

ฐ สี

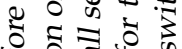

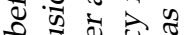

4 45

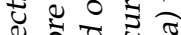

论

के $)$ व

से छ छ

m.

政

క0 ฐ

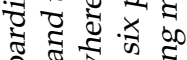

8.

ฐ

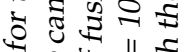

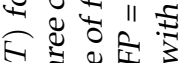

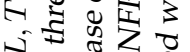

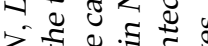

之吉志吉

๘

$\sum_{1} .70$.

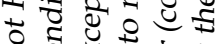

द. वे के के

की के के

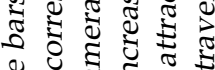

ฐ ฮี ฐ

$\mp$ हี 0 워

हี :

奈

की के क्ष

$\frac{\pi}{2}: 5$

ส

๘

के

4 ष

중

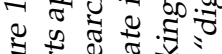

కี

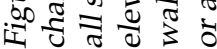

\begin{tabular}{|c|c|c|c|c|c|c|c|c|c|}
\hline \multicolumn{4}{|c|}{ PARTICIPANT KEY } & \multicolumn{2}{|c|}{\begin{tabular}{|l|l|} 
SET & SCENE \\
\end{tabular}} & \multirow{2}{*}{\begin{tabular}{|l|} 
CAMERA \\
PRO \\
\end{tabular}} & \multirow{2}{*}{\begin{tabular}{|l|} 
SET \\
T \\
\end{tabular}} & \multirow{2}{*}{\begin{tabular}{|l|} 
SCENE \\
CONCOURSE \\
\end{tabular}} & \multirow{2}{*}{\begin{tabular}{|l|} 
CAMERA \\
PRO \\
PPO
\end{tabular}} \\
\hline $\mathrm{A}=$ DIGITAL BARRIERS & $\mathrm{E}=$ NEUROTECHNOLOGY & $I=$ EYEDEA & $M=N E C$ & C & PHOTOJOURNALISM & & & & \\
\hline $\mathrm{B}=\mathrm{HBINNO}$ & $\mathrm{F}=$ VAPPLICA & $\mathrm{J}=$ HISIGN & $\mathrm{N}=$ TOSHIBA & $\mathrm{J}$ & PASSENGER LOADING & & & CONCOURSE & \\
\hline $\mathrm{C}=$ VIGILANT & $\mathrm{G}=\mathrm{MORPHO}$ & $\mathrm{K}=$ COGNITEC & $Q=$ IMAGUS & $\mathrm{P}$ & SPORTS ARENA & CONSUMER & $\mathrm{U}$ & CHOKEPOINT & WEBCAM \\
\hline $\mathrm{D}=$ AYONIX & $\mathrm{H}=3 \mathrm{M}$ COGENT & $\mathrm{L}=$ CYBEREXTRUDER & $\mathrm{R}=$ RANK ONE & $\mathrm{L}$ & LUGGAGE RACK & WEBCAM & & & \\
\hline
\end{tabular}



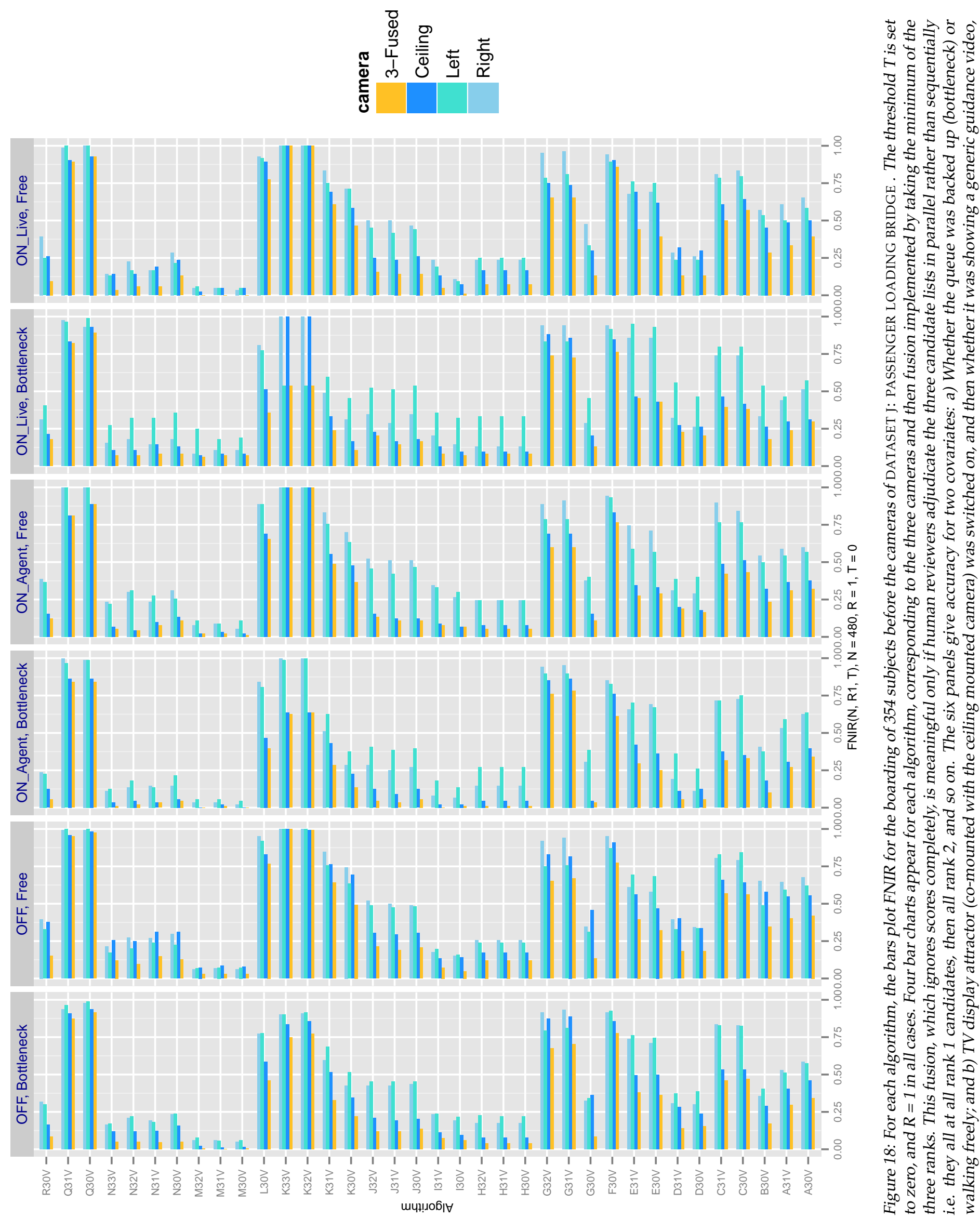

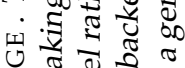

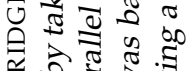

ต ส 3

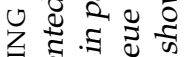

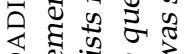

过:

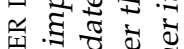

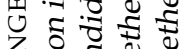

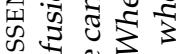

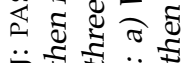

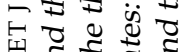

ए त స

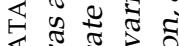

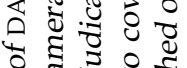

ช

ฮृ

ฐี ذँ

ฮิ

क

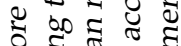

ปี

कิ ธี

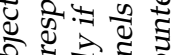

के

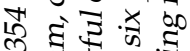

ชั

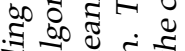

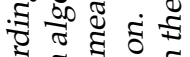

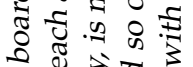

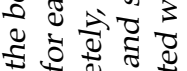

อั สี

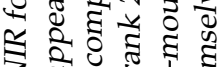

近

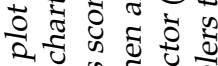

की

สี สี

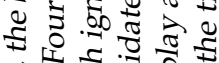

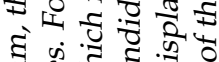

‡

ส

कำ สี โำ $\frac{1}{2}$

ส.

ชิ

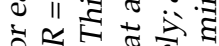

宁 $\frac{1}{0} \approx \frac{\pi}{8}$

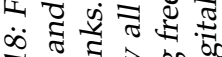

○

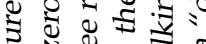

远

\begin{tabular}{|l|l|l|l|}
\hline \multicolumn{4}{|c|}{ PARTICIPANT KEY } \\
\hline $\mathrm{A}=$ DIGITAL BARRIERS & E = NEUROTECHNOLOGY & $\mathrm{I}=$ EYEDEA & $\mathrm{M}=$ NEC \\
\hline $\mathrm{B}=$ HBINNO & $\mathrm{F}=$ VAPPLICA & $\mathrm{J}=$ HISIGN & $\mathrm{N}=$ TOSHIBA \\
\hline $\mathrm{C}=$ VIGILANT & $\mathrm{G}=$ MORPHO & $\mathrm{K}=$ COGNITEC & $\mathrm{Q}=$ IMAGUS \\
\hline $\mathrm{D}=$ AYONIX & $\mathrm{H}=$ 3M COGENT & $\mathrm{L}=$ CYBEREXTRUDER & $\mathrm{R}=$ RANK ONE \\
\hline
\end{tabular}

\begin{tabular}{|l|l|l|l|l|l|}
\hline SET & SCENE & CAMERA & SET & SCENE & CAMERA \\
\hline C & PHOTOJOURNALISM & PRO & T & CONCOURSE & PRO \\
\hline J & PASSENGER LOADING & PRO & H & CONCOURSE & PRO \\
\hline P & SPORTS ARENA & CONSUMER & U & CHOKEPOINT & WEBCAM \\
\hline L & LUGGAGE RACK & WEBCAM & & & \\
\hline
\end{tabular}


on the results: Using a simple binomial assumption, this observed error rate only supports a claim that the error rate is below $10 \%$.

Multicamera fusion: We model the use of multiple cameras by taking for each known actor the maximum of the scores produced by searches from the three cameras - this gives the best "hit". This is fusion over space. Identification error rates are often lower when score fusion is conducted across the three cameras. Fusion is sometimes counterproductive because our metric, $\operatorname{FNIR}(T)$, is computed at a threshold that is computed as the 10-th largest of $n_{0}+n_{1}+n_{2}$ impostor scores, coming from cameras $\{0,1,2\}$. This is done because a system operator would not want to process more false positive exceptions just because he had installed two additional cameras. Algorithms vary in this respect depending on whether the two side cameras, with more yaw and less pitch, give high impostor scores.

The gains are usually modest relative to using just the ceiling mounted camera alone. This is especially true when the attractor is in use, and when the queue is stalled. Thus, multiple cameras give most benefit when an attractor is absent. This may be important if cameras are installed covertly without the possibility to use an attractor.

For the best-rank fusion method (Fig. 18), the FNIR values reach zero indicating that the actors were all identified correctly by at least one of the three cameras. This applies to algorithms from developers $\mathrm{M}$ and I, with $\mathrm{H}$ and $\mathrm{N}$ almost there. Given limited sample size, we again cannot claim error rates below about 3.5\% (via the rule of three). The use of best-rank fusion however is associated with increased labor costs, since for three cameras, three candidate lists will be produced (to first order) and the candidates will need to be interleaved and reviewed in rank-order.

Costs associated with fusion: Thus multiple cameras give most benefit when an attractor is absent, but their capital cost, and the costs associated with network transmission and computation, will increase linearly with the number of cameras. It is almost certainly less expensive therefore to deploy a capable attractor with eye catching and varied content, than it is to add additional cameras.

Caveats: Fielded accuracy will vary systematically from the numbers reported here. The equipment, illumination, and detailed installation details can have an effect. In addition the video here is being matched against still photographs collected on the same day. Such same-day matching is known to improve recognition accuracy. Note that some novel uses of face recognition do have a same-day concept of operations. One is to replace presentation of an airline boarding pass with one's face instead, using one-to-many recognition in a positive access control manner. This is only done after an initial identity check which includes a one-to-one verification of live imagery against an authoritative credential (e.g. passport).

\subsubsection{Effect of reduced frame rate}

The use of video data imparts a data size overhead. This arises because the pixel dimensions (e.g. 1920x1080) of the imagery are larger than face portraits (typically, 640x480) to support a useful field of view, and because video is typically collected at $24 \mathrm{fps}$ or 30fps. While video data is compressed using techniques that intelligently allow for interframe motion, the data rate is nevertheless large enough that a designer will need to make a dedicated computation of network bandwidth and latency needed to support operational goals.

\begin{tabular}{|l|l|l|l|}
\hline \multicolumn{5}{|c|}{ PARTICIPANT KEY } \\
\hline $\mathrm{A}=$ DIGITAL BARRIERS & $\mathrm{E}=$ NEUROTECHNOLOGY & $\mathrm{I}=$ EYEDEA & $\mathrm{M}=$ NEC \\
\hline $\mathrm{B}=$ HBINNO & $\mathrm{F}=$ VAPPLICA & $\mathrm{J}=$ HISIGN & $\mathrm{N}=$ TOSHIBA \\
\hline $\mathrm{C}=$ VIGILANT & $\mathrm{G}=$ MORPHO & $\mathrm{K}=$ COGNITEC & $\mathrm{Q}=$ IMAGUS \\
\hline $\mathrm{D}=$ AYONIX & $\mathrm{H}=$ 3M COGENT & $\mathrm{L}=$ CYBEREXTRUDER & $\mathrm{R}=$ RANK ONE \\
\hline
\end{tabular}

\begin{tabular}{|l|l|l|l|l|l|}
\hline SET & SCENE & CAMERA & SET & SCENE & CAMERA \\
\hline C & PHOTOJOURNALISM & PRO & T & CONCOURSE & PRO \\
\hline J & PASSENGER LOADING & PRO & H & CONCOURSE & PRO \\
\hline P & SPORTS ARENA & CONSUMER & U & CHOKEPOINT & WEBCAM \\
\hline L & LUGGAGE RACK & WEBCAM & & & \\
\hline
\end{tabular}



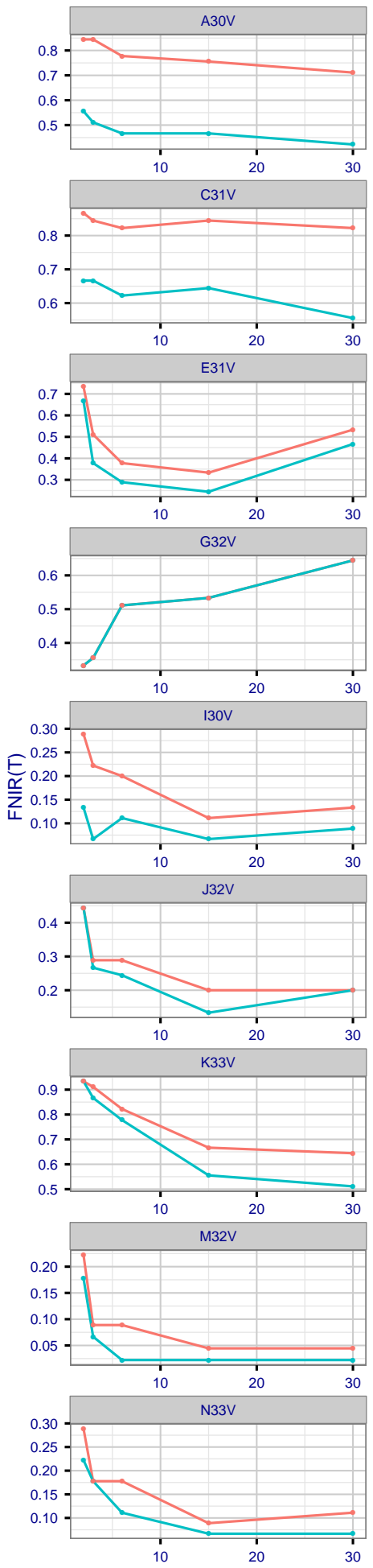
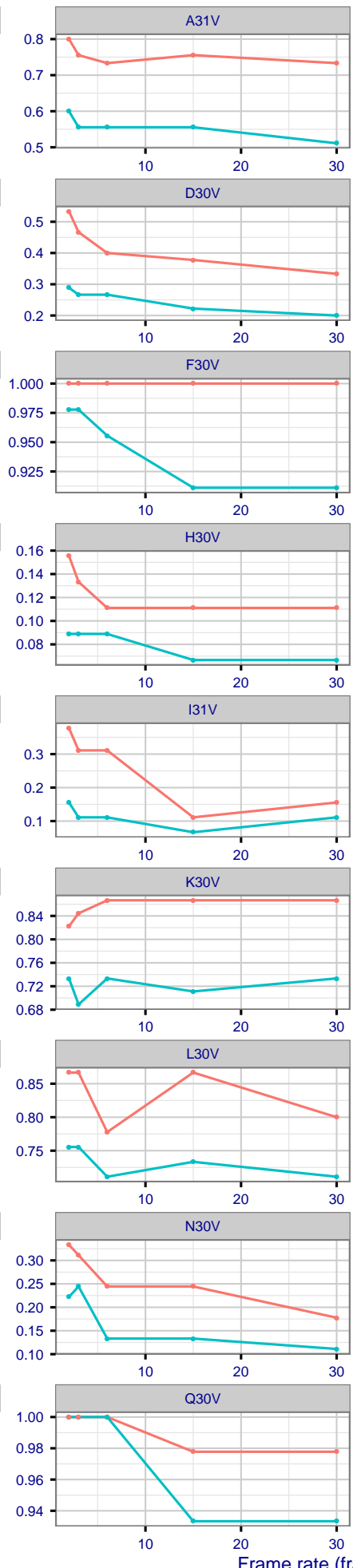

Frame rate (fra
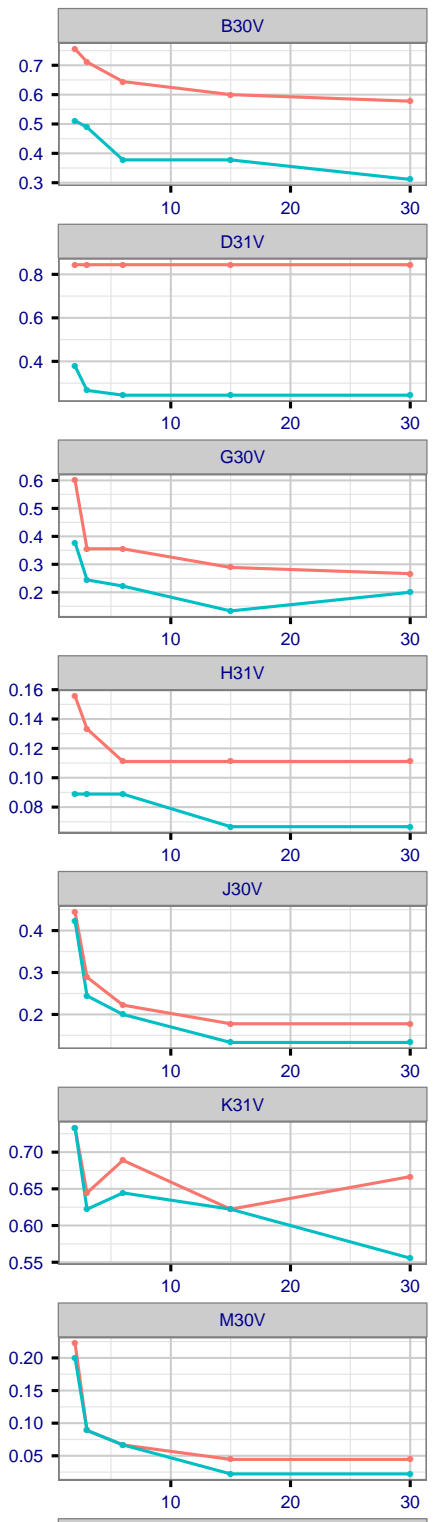

N31V
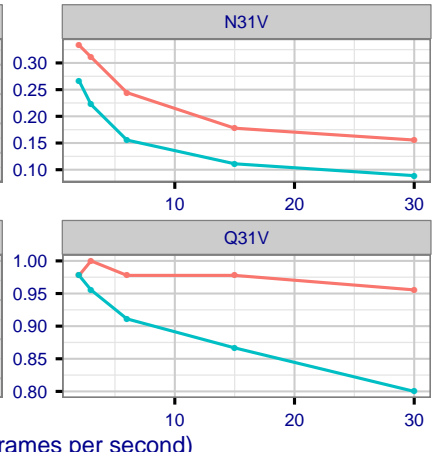
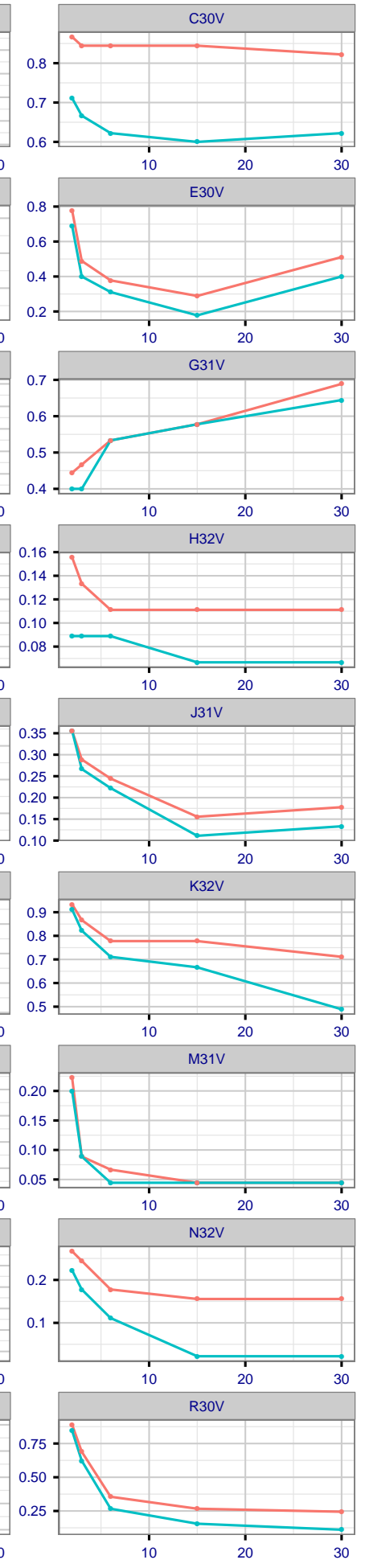

Figure 19: Using a subset of the DATASET J: PASSENGER LOADING BRIDGE imagery, the panels show $F N I R(N, L, T)$ against frame rate. The algorithms were given video sequences with progressively reduced temporal resolution. This was achieved by using every $k$-th frame as input, $k=\{1,2,5,10,15\}$, from an original frame rate of $30 \mathrm{fps}$. The recognition threshold, $T$, was set to achieve 10 and 100 false positives over identification searches into a gallery of size $N=480$. Thresholds are set specifically for each frame rate. The camera is ceiling mounted. The subjects walk freely, and a video attractor was present. Note the different vertical scales. Note also this figure's use of lines is possibly erroneous as we did not measure accuracy at all possible frames rates, only those stated.

\begin{tabular}{|l|l|l|l|}
\hline \multicolumn{4}{|c|}{ PARTICIPANT KEY } \\
\hline A = DIGITAL BARRIERS & E = NEUROTECHNOLOGY & I = EYEDEA & $\mathrm{M}=$ NEC \\
\hline $\mathrm{B}=$ HBINNO & F = VAPPLICA & $\mathrm{J}=$ HISIGN & $\mathrm{N}=$ TOSHIBA \\
\hline C = VIGILANT & G = MORPHO & $\mathrm{K}=$ COGNITEC & $\mathrm{Q}=$ IMAGUS \\
\hline $\mathrm{D}=$ AYONIX & $\mathrm{H}=$ 3M COGENT & $\mathrm{L}=$ CYBEREXTRUDER & $\mathrm{R}=$ RANK ONE \\
\hline
\end{tabular}

\begin{tabular}{|l|l|l|l|l|l|}
\hline SET & SCENE & CAMERA & SET & SCENE & CAMERA \\
\hline C & PHOTOJOURNALISM & PRO & T & CONCOURSE & PRO \\
\hline J & PASSENGER LOADING & PRO & H & CONCOURSE & PRO \\
\hline P & SPORTS ARENA & CONSUMER & U & CHOKEPOINT & WEBCAM \\
\hline L & LUGGAGE RACK & WEBCAM & & & \\
\hline
\end{tabular}




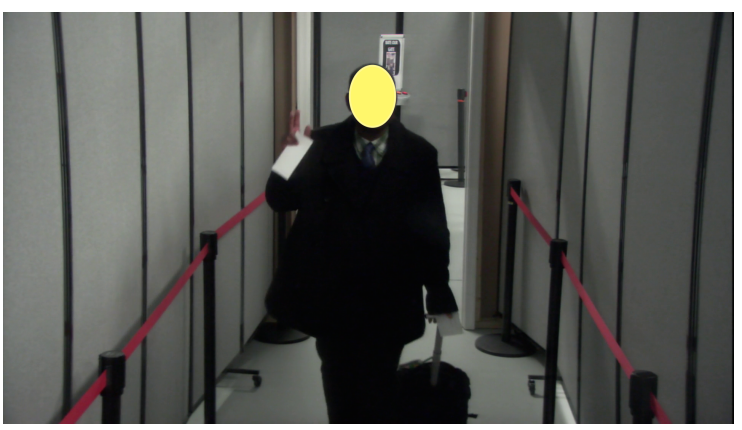

(a) Free Movement Scenario

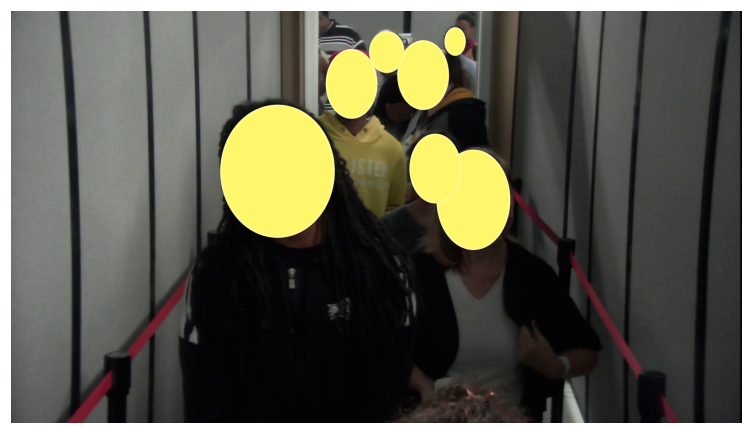

(b) Bottlenecked Scenario

Figure 20: Example frames for the Free Movement (left) and Bottlenecked (right) scenarios from the DATASET J: PASSENGER LOADING BRIDGE dataset. The yellow ellipses are applied as these individuals did not consent for their faces to appear in the report.

As video data is typically much larger than still imagery, the question of how to reduce the data size arises. This can be achieved in a number of ways. First, is to not transmit the video at all, and instead extract features in or near the camera. This "edge processing" mode of operations requires installation of some components of a face recognition system at, or near, the camera (rather than at a central server), and this in turn requires fielding of sufficient computation power also. It binds the camera to the algorithm and may make technology update more difficult. Second, is a hybrid solution where video of detected faces is transmitted. This requires face detection algorithms to be fielded. Third, is to reduce the bit-rate by using a different compression profile or (not exactly equivalently) by reducing the frame rate. This last aspect is analyzed here.

We presented results for DATASET J: PASSENGER LOADING BRIDGE at full frame rate above. We additionally searched the same video at reduced frame rates. This was done by passing only every $\mathrm{k}$-th frame to the algorithms, with $k=$ $\{15,10,5,2,1\}$, the last value representing the full 30 frames per second (fps). As algorithms use motion to detect, and track, and potentially integrate information over time, any reduction in frame rate can undermine accuracy.

Figure 19 shows the effect of reduced frame rate on $\operatorname{FNIR}(N, L, T)$, i.e. the proportion of actors not identified above a threshold $\mathrm{T}$. The threshold $\mathrm{T}$ was set to give $\mathrm{NFP}=\{10,100\}$ false positives at each frame rate. The results are varied. Several algorithms - those from providers, B, D, H, I, M, N, Q, R - mostly give the expected behavior: better accuracy with more video data. However, some algorithms give entirely the opposite: G31V and G32V have substantially better accuracy with two frames per second than at higher frame rates. The G30V algorithm is usually superior but G32V is more effective at 2 frames per second. G31V and G32V produce larger templates more slowly. The algorithms from participants E, I, J mostly give best accuracy at $15 \mathrm{fps}$. Some other algorithms, from developers $\mathrm{H}$ and $\mathrm{M}$, seem to operate at 15 fps naturally.

The data support a conclusion that operating at $15 \mathrm{fps}$ is often lossless. A weakness of this study however is that we have not measured the attendant data size gains: While there are half as many frames, the compression algorithm may not realize such a reduction because interframe subject motion is larger at $15 \mathrm{fps}$ than at $30 \mathrm{fps}$.

\subsubsection{Face tracking behavior}

A preliminary step in the identification process involves detecting and tracking the movement of a person across multiple frames. These face tracks are then processed into matchable templates. A single face track need not correspond to the

\begin{tabular}{|l|l|l|l|}
\hline \multicolumn{5}{|c|}{ PARTICIPANT KEY } \\
\hline $\mathrm{A}=$ DIGITAL BARRIERS & $\mathrm{E}=$ NEUROTECHNOLOGY & $\mathrm{I}=$ EYEDEA & $\mathrm{M}=$ NEC \\
\hline $\mathrm{B}=$ HBINNO & $\mathrm{F}=$ VAPPLICA & $\mathrm{J}=$ HISIGN & $\mathrm{N}=$ TOSHIBA \\
\hline $\mathrm{C}=$ VIGILANT & $\mathrm{G}=$ MORPHO & $\mathrm{K}=$ COGNITEC & $\mathrm{Q}=$ IMAGUS \\
\hline $\mathrm{D}=$ AYONIX & $\mathrm{H}=$ 3M COGENT & $\mathrm{L}=$ CYBEREXTRUDER & $\mathrm{R}=$ RANK ONE \\
\hline
\end{tabular}

\begin{tabular}{|l|l|l|l|l|l|}
\hline SET & SCENE & CAMERA & SET & SCENE & CAMERA \\
\hline C & PHOTOJOURNALISM & PRO & T & CONCOURSE & PRO \\
\hline J & PASSENGER LOADING & PRO & H & CONCOURSE & PRO \\
\hline P & SPORTS ARENA & CONSUMER & U & CHOKEPOINT & WEBCAM \\
\hline L & LUGGAGE RACK & WEBCAM & & & \\
\hline
\end{tabular}


entire duration that a person is in the field of view of the camera. For example, if a person's face is only visible at sporadic moments (due to temporary occlusion or changes in head pose), the algorithm may decide to generate a separate face track for each continuous period over which the face is visible.

Figure 20 shows an example snapshot for two scenarios from DATASET J: PASSENGER LOADING BRIDGE . For the "Free Movement" scenario on the left, subjects walked unimpeded down the PLB, in most cases presenting their faces to the camera without interruption. For the "Bottlenecked" scenario on the right, a queue formed. People in the Bottlenecked scenario are typically in the field-of-view for a longer duration, but their behavior is less consistent. They often look down or away from the camera, and it is not uncommon for their faces to be temporarily occluded by the people waiting in front of them.

Figure 21 shows the number of face tracks detected for each algorithm and both scenarios. The number of detected face tracks varied widely from one algorithm to the next. Even for the free movement scenario, algorithm D30V reported about 13 times more face tracks than people who walked down the PLB. The algorithm is probably breaking each person's presentation into multuple face tracks, despite uninterrupted presentation of the face. One could argue that this gives the algorithm a greater number of opportunities to "hit" the person in the database, since we give an algorithm credit for a hit as long as it found the person at least once while he/she was walking down the aisle (more face tracks means more search templates means more opportunities to hit). On the other hand, it could lead to a greater number of false positives. Ideally, the algorithm would detect one face track per person walking down the PLB (although this behavior was not specifically requested in the API).

Algorithms G31V and G32V detect fewer faces in the bottlenecked scenario. Fewer, in fact, than there are people in the video, indicating either a failure to track certain individuals, or incorrect consolidation of several different people into a single face track.

Figure 22 supplements Figure 21 by showing the mean face track length (in seconds) compared to the number of face tracks found for each algorithm. This figure applies only to freely walking subjects. The notable results are:

As expected, there is an inverse relationship between face track length and number of face tracks.

Notably six of the more accurate algorithms are clustered right and below center. The majority of the more accurate algorithms (e.g. those from providers, H, I, J, M, N, G) track subjects for several seconds, and produce up to two times the number of tracks as there are people.

However for the long clip video over which these results were reported, the G31V and G32V algorithms report fewer tracks than there are people, which is fatal to FNIR.

Some algorithms (for example from providers A, C, D, F, L, R, Q) elect to track faces over short sub-second intervals but also report more tracks. Algorithm F30V finds many face tracks, but each face track spans an average of less than a fifth of a second. Since it typically takes a subject several seconds to walk down the PLB, the algorithm is likely breaking up the person's journey across several face tracks.

On the other hand, the K31V algorithm reports tracks much longer than the subjects are actually in view. The developer explored this tradespace with K30V producing many short tracks and K33V producing face tracks that span an average of 6.2 seconds, more in line with how long the person is actually in the field of view.

\begin{tabular}{|l|l|l|l|}
\hline \multicolumn{4}{|c|}{ PARTICIPANT KEY } \\
\hline $\mathrm{A}=$ DIGITAL BARRIERS & $\mathrm{E}=$ NEUROTECHNOLOGY & $\mathrm{I}=$ EYEDEA & $\mathrm{M}=$ NEC \\
\hline $\mathrm{B}=$ HBINNO & $\mathrm{F}=$ VAPPLICA & $\mathrm{J}=$ HISIGN & $\mathrm{N}=$ TOSHIBA \\
\hline $\mathrm{C}=$ VIGILANT & $\mathrm{G}=$ MORPHO & K = COGNITEC & $\mathrm{Q}=$ IMAGUS \\
\hline $\mathrm{D}=$ AYONIX & $\mathrm{H}=$ 3M COGENT & $\mathrm{L}=$ CYBEREXTRUDER & $\mathrm{R}=$ RANK ONE \\
\hline
\end{tabular}

\begin{tabular}{|l|l|l|l|l|l|}
\hline SET & SCENE & CAMERA & SET & SCENE & CAMERA \\
\hline C & PHOTOJOURNALISM & PRO & T & CONCOURSE & PRO \\
\hline J & PASSENGER LOADING & PRO & H & CONCOURSE & PRO \\
\hline P & SPORTS ARENA & CONSUMER & U & CHOKEPOINT & WEBCAM \\
\hline L & LUGGAGE RACK & WEBCAM & & & \\
\hline
\end{tabular}



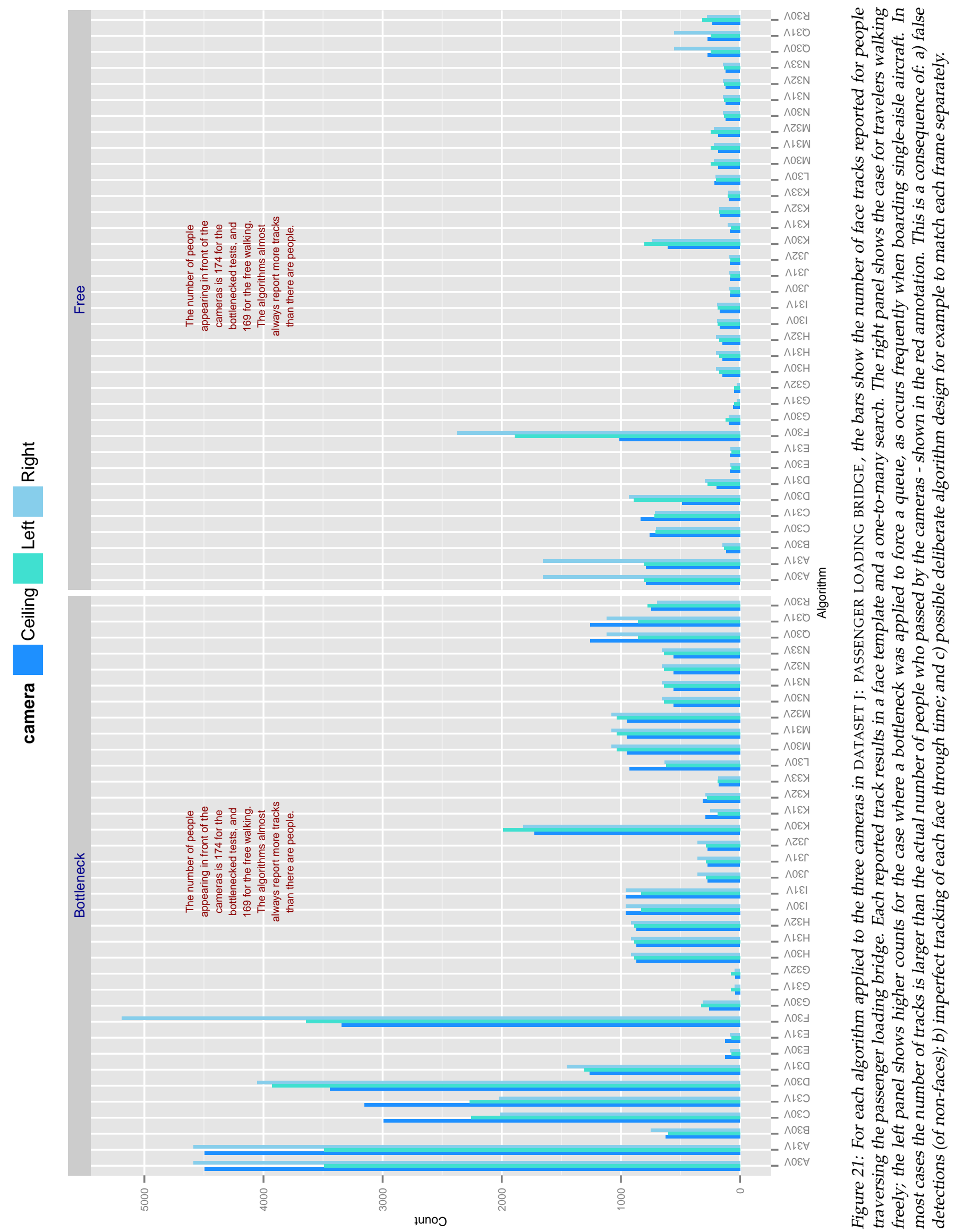

\begin{tabular}{|l|l|l|l|}
\hline \multicolumn{4}{|c|}{ PARTICIPANT KEY } \\
\hline A = DIGITAL BARRIERS & E = NEUROTECHNOLOGY & I = EYEDEA & $\mathrm{M}=$ NEC \\
\hline B = HBINNO & F = VAPPLICA & I HISIGN & $\mathrm{N}=$ TOSHIBA \\
\hline C = VIGILANT & G = MORPHO & K = COGNITEC & $\mathrm{Q}=$ IMAGUS \\
\hline D = AYONIX & $\mathrm{H}=$ 3M COGENT & $\mathrm{L}=$ CYBEREXTRUDER & $\mathrm{R}=$ RANK ONE \\
\hline
\end{tabular}

\begin{tabular}{|l|l|l|l|l|l|}
\hline SET & SCENE & CAMERA & SET & SCENE & CAMERA \\
\hline C & PHOTOJOURNALISM & PRO & T & CONCOURSE & PRO \\
\hline J & PASSENGER LOADING & PRO & H & CONCOURSE & PRO \\
\hline P & SPORTS ARENA & CONSUMER & U & CHOKEPOINT & WEBCAM \\
\hline L & LUGGAGE RACK & WEBCAM & & & \\
\hline
\end{tabular}




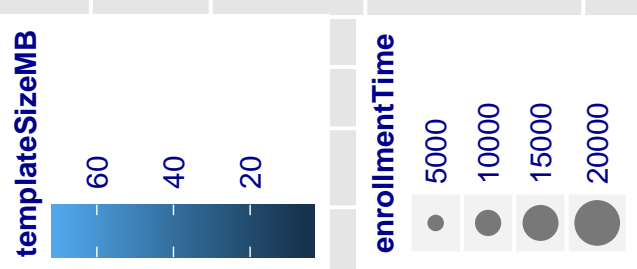

-

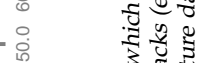

0 -

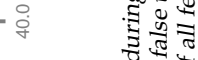

के

范

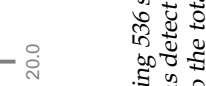

ฐ

a

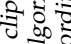

कृ

.

-

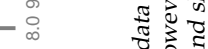

-

-0 家它

(1)

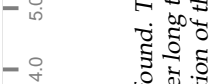

की

-

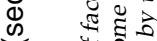

व के

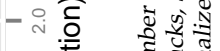

元 䎡

을

苛 के

-

-

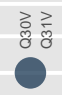

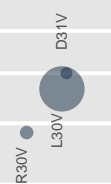

$-{ }_{0}^{\infty}=$

- 0 ठ

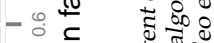

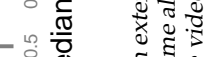

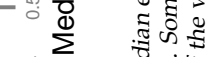

ฐัฐ

ฐ ฐี : ฐี

응

,

$-\stackrel{+}{\circ}$

욤

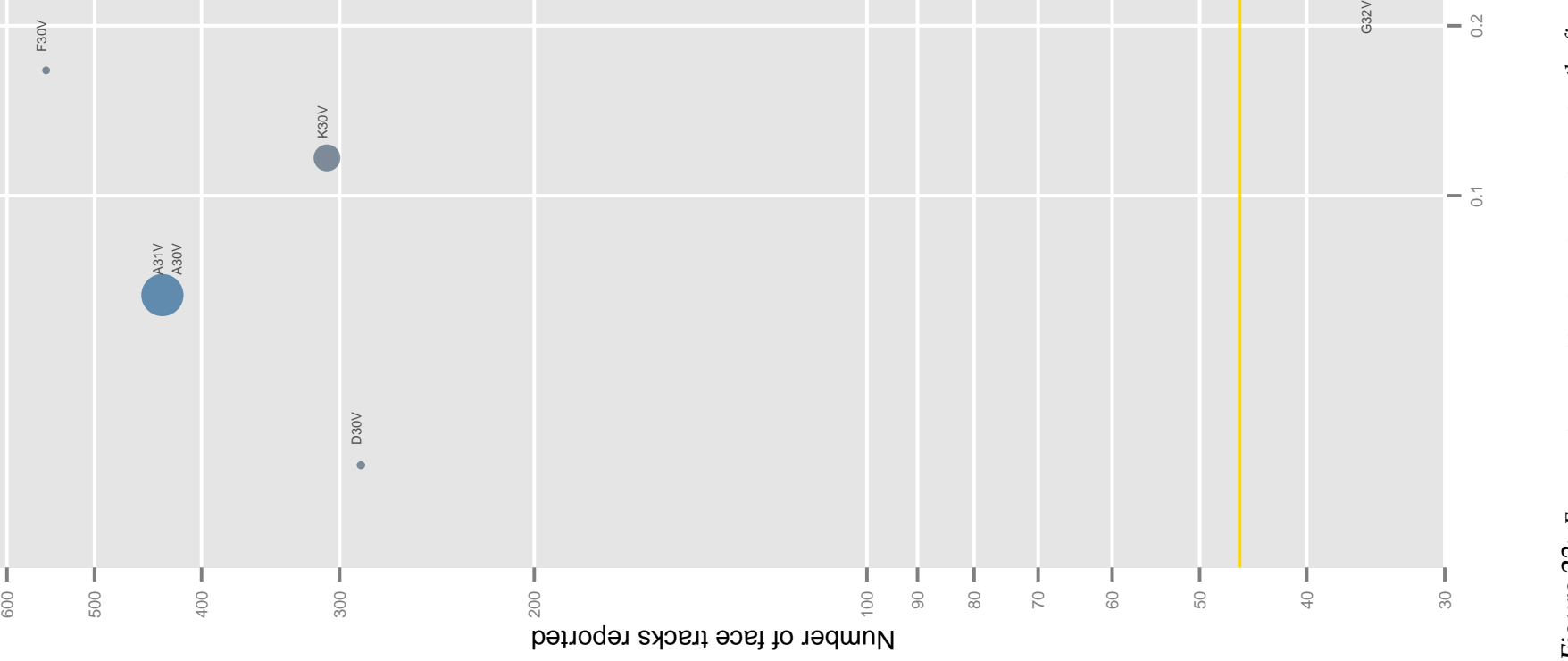

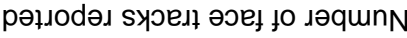

PARTICIPANT KEY

\begin{tabular}{|l|l|l|l|}
\hline $\mathrm{A}=$ DIGITAL BARRIERS & $\mathrm{E}=$ NEUROTECHNOLOGY & $\mathrm{I}=$ EYEDEA & $\mathrm{M}=$ NEC \\
\hline $\mathrm{B}=$ HBINNO & $\mathrm{F}=$ VAPPLICA & $\mathrm{J}=$ HISIGN & $\mathrm{N}=$ TOSHIBA \\
\hline $\mathrm{C}=$ VIGILANT & $\mathrm{G}=$ MORPHO & $\mathrm{K}=$ COGNITEC & $\mathrm{Q}=$ IMAGUS \\
\hline $\mathrm{D}=$ AYONIX & $\mathrm{H}=$ 3M COGENT & $\mathrm{L}=$ CYBEREXTRUDER & $\mathrm{R}=$ RANK ONE \\
\hline
\end{tabular}

\begin{tabular}{|l|l|l|l|l|l|}
\hline SET & SCENE & CAMERA & SET & SCENE & CAMERA \\
\hline C & PHOTOJOURNALISM & PRO & T & CONCOURSE & PRO \\
\hline J & PASSENGER LOADING & PRO & H & CONCOURSE & PRO \\
\hline P & SPORTS ARENA & CONSUMER & U & CHOKEPOINT & WEBCAM \\
\hline L & LUGGAGE RACK & WEBCAM & & & \\
\hline
\end{tabular}




\subsubsection{Viable spatial resolution}

Figure 23 leverages spatial track information reported by the algorithms to show at what resolutions ${ }^{13}$ faces are being aquired and tracked through video clips. This was generated over 29118 seconds of video, collected at 30 frames per second, using three cameras yielding 48 clips.

Regarding the resolution at which algorithms first acquire subjects, at a minimum resolution, and then cease tracking, the following observations are notable.

Effect of walking: As is visible in Figure 23, most algorithms detect freely walking individuals earlier and track them longer than if the subject is standing in a queue. This occurs because bottlenecked individuals are often occluded.

Note the interocular distances given in Figure 23 are medians, and there could be considerable and differing variances around these figures.

Algorithms vary in minimum resolution requirement: One algorithm, L30V, acquires and reports faces at essentially zero resolution. Whether features are extracted from low resolution faces is not evident in a black box study. However, beyond this, some algorithms appear to be configured to only acquire faces with at least 20 (participants, A, K, M), 30 (C, D), $40(\mathrm{D}, \mathrm{R})$ or $50(\mathrm{~F})$ pixels between the eyes.

Algorithms vary in maximum resolution: Some algorithms (participants B, E, H, N) acquire and report faces from freely walking subjects with median 120 pixels or higher interocular distance. This high resolution occurs when subjects are close to the camera, usually with the most advserse pose angle (high pitch or yaw). Whether features are extracted from these frames can not be determined given this is a black box test. Other algorithms, notably the most accurate $\mathrm{M}$ algorithms cease to report face tracks with IOD beyond 80 pixels.

\subsubsection{Template sizes}

Figure 24 shows the size of template data extracted from 30 frames per second video data. Size is reported as a function of: a) whether subjects were queued or walking freely; b) camera placement (ceiling-vs. wall-mounted); c) whether the ceiling-mounted attractor was on or off.

The median amount of data extracted from a video track, the template size, varies massively between algorithms ranging over three orders of magnitude from hundreds of bytes up to more than a million. For most algorithms the median varies little with the three factors.

\footnotetext{
${ }^{13}$ The term resolution here follows common practice as being a synonym for spatial sampling rate - a measurement in pixels. It is more properly reserved for optical resolution which is measured by using dedicated tests and reported, often, by stating the modulation of intensity values at some spatial frequency. Resolution includes the effects of poor lenses, compression, and atmospheric distortion. It is possible to have many pixels on a poorly resolved target.
}

\begin{tabular}{|l|l|l|l|}
\hline \multicolumn{5}{|c|}{ PARTICIPANT KEY } \\
\hline $\mathrm{A}=$ DIGITAL BARRIERS & $\mathrm{E}=$ NEUROTECHNOLOGY & $\mathrm{I}=$ EYEDEA & $\mathrm{M}=$ NEC \\
\hline $\mathrm{B}=$ HBINNO & $\mathrm{F}=$ VAPPLICA & $\mathrm{J}=$ HISIGN & $\mathrm{N}=$ TOSHIBA \\
\hline $\mathrm{C}=$ VIGILANT & $\mathrm{G}=$ MORPHO & $\mathrm{K}=$ COGNITEC & $\mathrm{Q}=$ IMAGUS \\
\hline $\mathrm{D}=$ AYONIX & $\mathrm{H}=$ 3M COGENT & $\mathrm{L}=$ CYBEREXTRUDER & $\mathrm{R}=$ RANK ONE \\
\hline
\end{tabular}

\begin{tabular}{|l|l|l|l|l|l|}
\hline SET & SCENE & CAMERA & SET & SCENE & CAMERA \\
\hline C & PHOTOJOURNALISM & PRO & T & CONCOURSE & PRO \\
\hline J & PASSENGER LOADING & PRO & H & CONCOURSE & PRO \\
\hline P & SPORTS ARENA & CONSUMER & U & CHOKEPOINT & WEBCAM \\
\hline L & LUGGAGE RACK & WEBCAM & & & \\
\hline
\end{tabular}



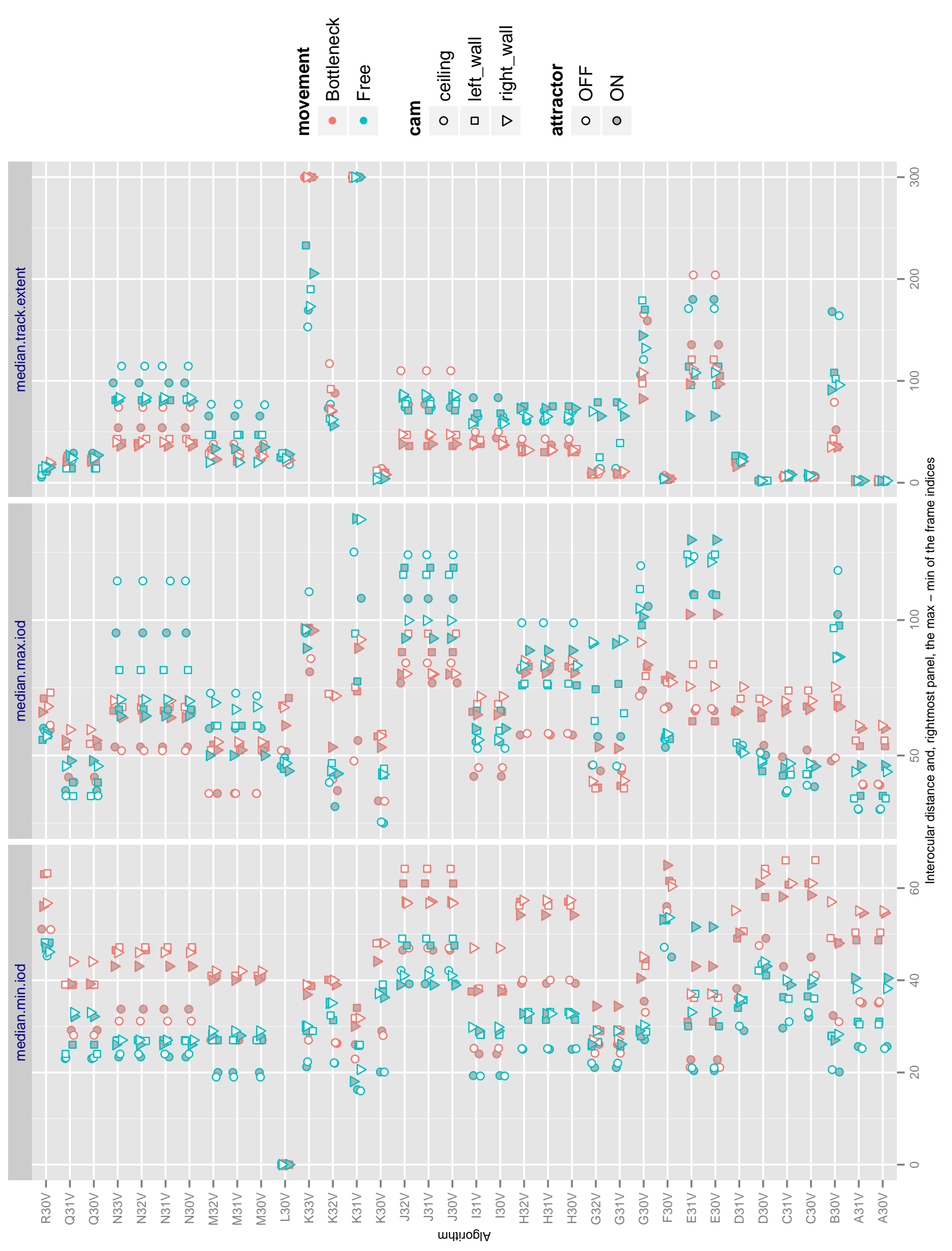

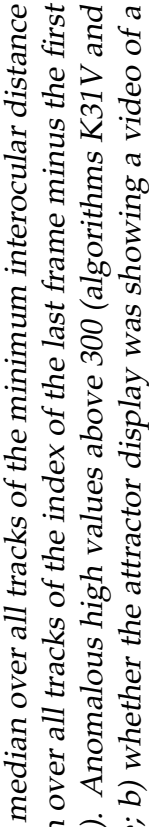

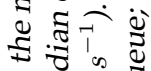
สิ छั के శ్ 菅步. का 0 त ฐ ฐ छี สั ฐ ฐ ㄱ: 政完 范

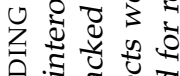
宩 $₫$ $\approx$ घ 5 के

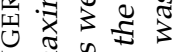

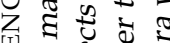
思 ब के के वै $\ddot{\sigma}$ 歌歌

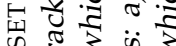
$\mathbb{4} \pm$

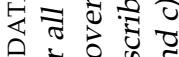
0 むั c 0 \% व की ฉ: ซี. ส ฐี ฐ $\Xi$ : ส $\frac{0}{\pi} \pi$ ป क

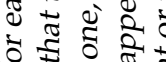

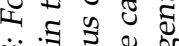
$\ddot{\sim} \approx \frac{7}{\pi} \approx$

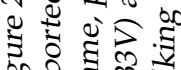

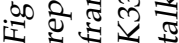

\begin{tabular}{|c|c|c|c|c|c|c|c|c|c|}
\hline \multicolumn{4}{|c|}{ PARTICIPANT KEY } & \multirow{2}{*}{\begin{tabular}{|l|} 
SET \\
$C$ \\
\end{tabular}} & SCENE & \multirow{2}{*}{\begin{tabular}{|l|} 
CAMERA \\
PRO \\
\end{tabular}} & \multirow{2}{*}{\begin{tabular}{|l} 
SET \\
T \\
\end{tabular}} & \multirow{2}{*}{\begin{tabular}{|l|l|} 
SCENE \\
CONCOURSE
\end{tabular}} & \multirow{2}{*}{$\begin{array}{l}\text { CAMERA } \\
\text { PRO }\end{array}$} \\
\hline $\mathrm{A}=$ DIGITAL BARRIERS & $\mathrm{E}=\mathrm{NEUROTECHNOLOGY}$ & $I=$ EYEDEA & $M=N E C$ & & PHOTOJOURNALISM & & & & \\
\hline $\mathrm{C}=$ VIGILANT & $G=M O R P H O$ & $\mathrm{~K}=$ COGNITEC & $\mathrm{Q}=$ IMAGUS & $\mathrm{P}$ & SPORTS ARENA & CONSUMER & $\mathrm{U}$ & CHOKEPOINT & WEBCAM \\
\hline $\mathrm{D}=\mathrm{AYONIX}$ & $\mathrm{H}=3 \mathrm{M}$ COGENT & $\mathrm{L}=$ CYBEREXTRUDER & $\mathrm{R}=\mathrm{RANK}$ ONE & $\mathrm{L}$ & LUGGAGE RACK & WEBCAM & & & \\
\hline
\end{tabular}



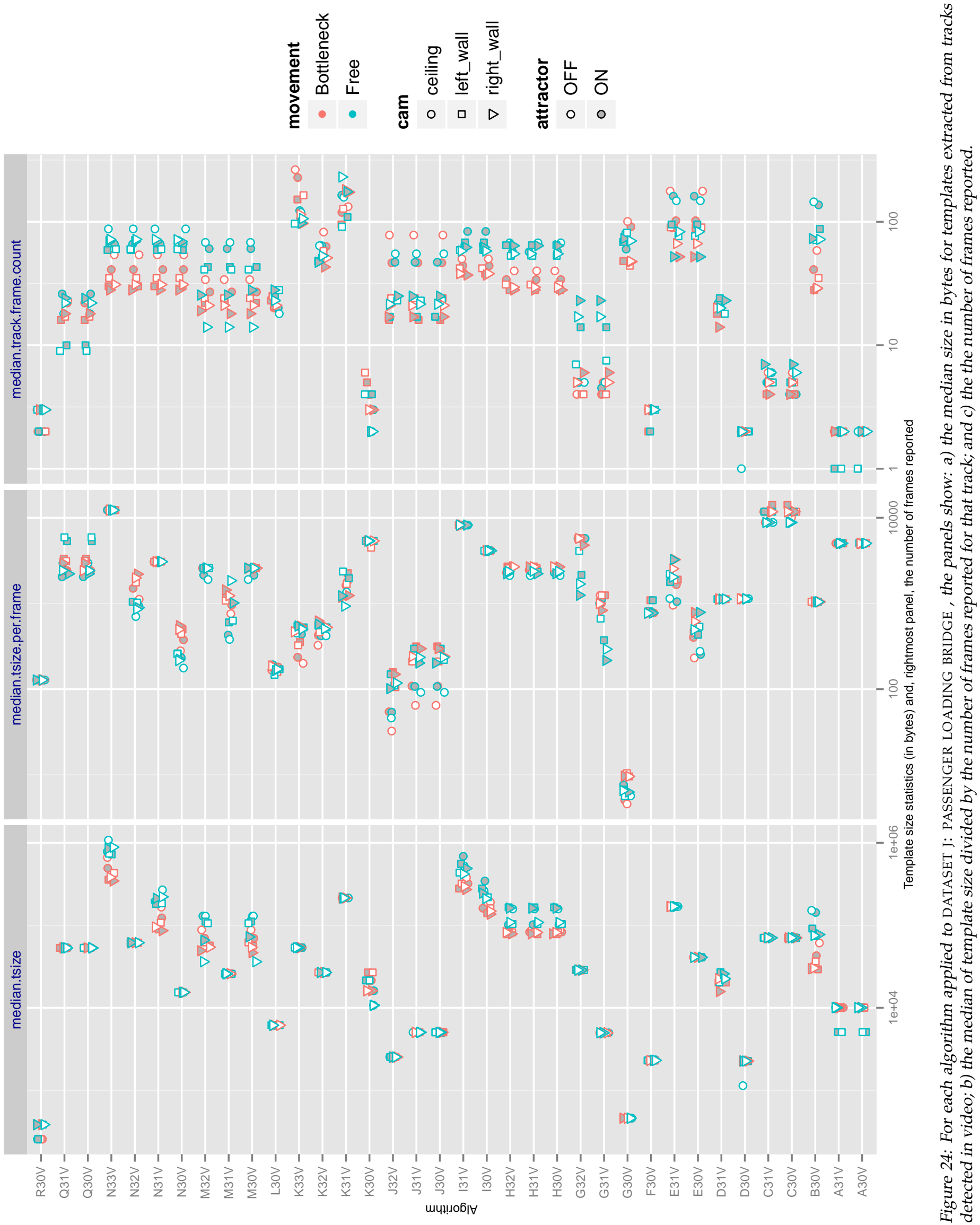

\begin{tabular}{|l|l|l|l|}
\hline \multicolumn{4}{|c|}{ PARTICIPANT KEY } \\
\hline $\mathrm{A}=$ DIGITAL BARRIERS & $\mathrm{E}=$ NEUROTECHNOLOGY & $\mathrm{I}=$ EYEDEA & $\mathrm{M}=$ NEC \\
\hline $\mathrm{B}=$ HBINNO & $\mathrm{F}=$ VAPPLICA & $\mathrm{J}=$ HISIGN & $\mathrm{N}=$ TOSHIBA \\
\hline $\mathrm{C}=$ VIGILANT & $\mathrm{G}=$ MORPHO & $\mathrm{K}=$ COGNITEC & $\mathrm{Q}=$ IMAGUS \\
\hline $\mathrm{D}=$ AYONIX & $\mathrm{H}=$ MM COGENT & $\mathrm{L}=$ CYBEREXTRUDER & $\mathrm{R}=$ RANK ONE \\
\hline
\end{tabular}

\begin{tabular}{|l|l|l|l|l|l|}
\hline SET & SCENE & CAMERA & SET & SCENE & CAMERA \\
\hline C & PHOTOJOURNALISM & PRO & T & CONCOURSE & PRO \\
\hline J & PASSENGER LOADING & PRO & H & CONCOURSE & PRO \\
\hline P & SPORTS ARENA & CONSUMER & U & CHOKEPOINT & WEBCAM \\
\hline L & LUGGAGE RACK & WEBCAM & & & \\
\hline
\end{tabular}



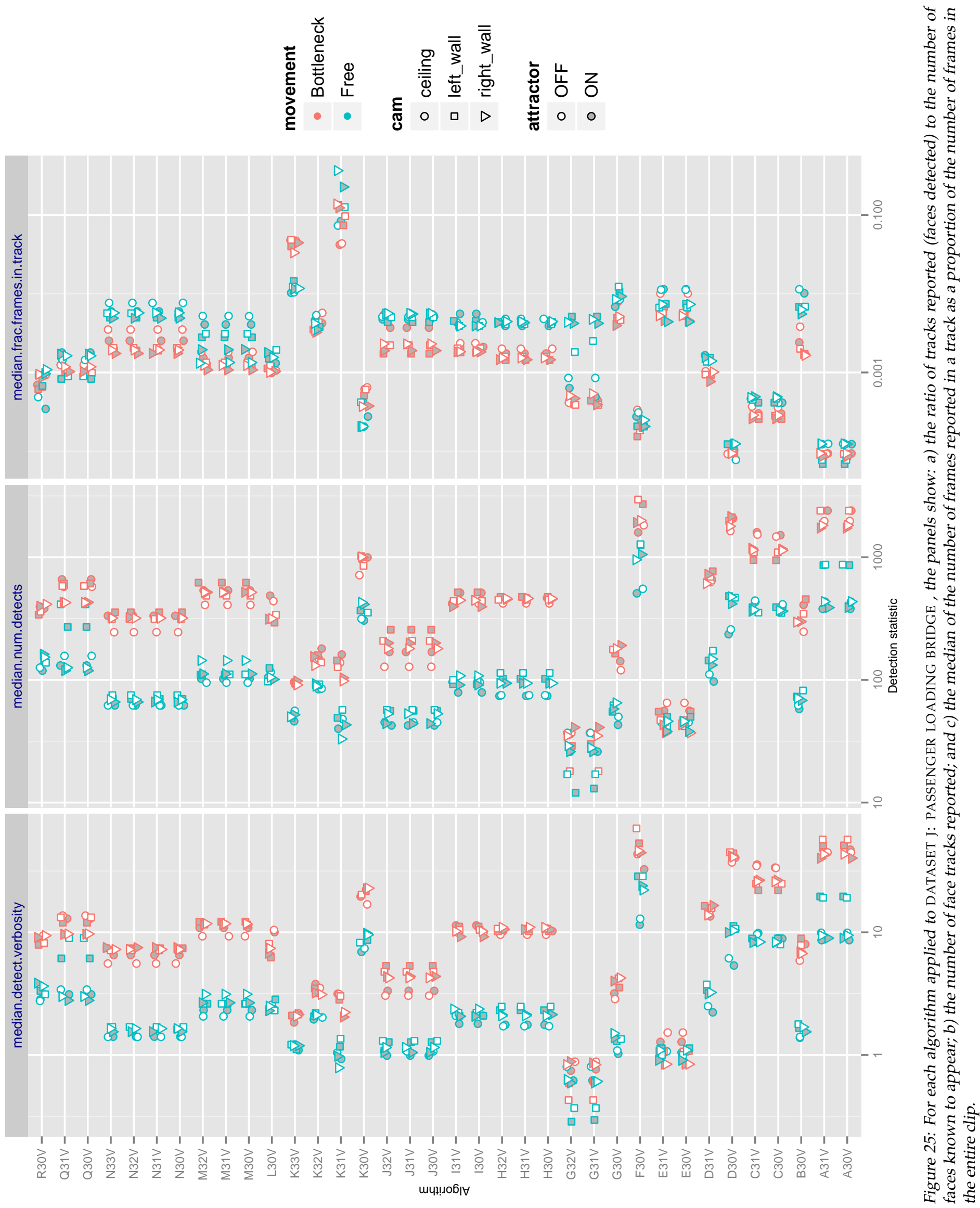

\begin{tabular}{|l|l|l|l|}
\hline \multicolumn{4}{|c|}{ PARTICIPANT KEY } \\
\hline $\mathrm{A}=$ DIGITAL BARRIERS & $\mathrm{E}=$ NEUROTECHNOLOGY & $\mathrm{I}=$ EYEDEA & $\mathrm{M}=$ NEC \\
\hline $\mathrm{B}=$ HBINNO & $\mathrm{F}=$ VAPPLICA & $\mathrm{J}=$ HISIGN & $\mathrm{N}=$ TOSHIBA \\
\hline $\mathrm{C}=$ VIGILANT & $\mathrm{G}=$ MORPHO & $\mathrm{K}=$ COGNITEC & $\mathrm{Q}=$ IMAGUS \\
\hline $\mathrm{D}=$ AYONIX & $\mathrm{H}=3 \mathrm{M}$ COGENT & $\mathrm{L}=$ CYBEREXTRUDER & $\mathrm{R}=$ RANK ONE \\
\hline
\end{tabular}

\begin{tabular}{|l|l|l|l|l|l|}
\hline SET & SCENE & CAMERA & SET & SCENE & CAMERA \\
\hline C & PHOTOJOURNALISM & PRO & T & CONCOURSE & PRO \\
\hline J & PASSENGER LOADING & PRO & H & CONCOURSE & PRO \\
\hline P & SPORTS ARENA & CONSUMER & U & CHOKEPOINT & WEBCAM \\
\hline L & LUGGAGE RACK & WEBCAM & & & \\
\hline
\end{tabular}




\begin{tabular}{|c|c|c|c|c|c|c|c|c|}
\hline DATASET J & \multicolumn{6}{|c|}{ VIDEO SEARCH } & \multirow{2}{*}{\multicolumn{2}{|c|}{$\begin{array}{l}\text { STILL IMAGE } \\
\text { IOD (PIXELS) }\end{array}$}} \\
\hline & NUMBER OF & MEAN TRACK & MEAN TRACK & MEAN & MEAN & MEAN & & \\
\hline ALGORITHM & TRACKS & LENGTH (FRAMES) & EXTENT (FRAMES) & MIN IOD (PX) & MEAN IOD (PX) & MAX IOD (PX) & MEAN & STD. DEV \\
\hline $\mathrm{A} 30 \mathrm{~V}$ & 63317 & 15 & 15 & 51 & 54 & 57 & 116 & 20 \\
\hline A31V & 63317 & 15 & 15 & 51 & 54 & 57 & 116 & 20 \\
\hline B30V & 9533 & 117 & 139 & 50 & 65 & 131 & 116 & 17 \\
\hline $\mathrm{C} 30 \mathrm{~V}$ & 37758 & 12 & 13 & 58 & 62 & 67 & 117 & 20 \\
\hline C31V & 38860 & 12 & 13 & 57 & 61 & 66 & 117 & 20 \\
\hline D30V & 54933 & 16 & 16 & 64 & 67 & 70 & 117 & 17 \\
\hline D31V & 19168 & 56 & 56 & 53 & 63 & 73 & 117 & 17 \\
\hline E30V & 2081 & 170 & 199 & 40 & 59 & 99 & 116 & 17 \\
\hline E31V & 2081 & 170 & 199 & 40 & 59 & 99 & 116 & 17 \\
\hline F30V & 69839 & 6 & 63 & 65 & 69 & 74 & 115 & 17 \\
\hline G30V & 3947 & 169 & 288 & 46 & 64 & 95 & 116 & 18 \\
\hline G31V & 1167 & 784 & 1004 & 37 & 50 & 78 & 116 & 18 \\
\hline G32V & 1167 & 783 & 1003 & 37 & 50 & 78 & 116 & 18 \\
\hline H30V & 12788 & 58 & 62 & 56 & 68 & 88 & 118 & 17 \\
\hline H31V & 12788 & 58 & 62 & 56 & 68 & 88 & 118 & 17 \\
\hline H32V & 12788 & 58 & 62 & 56 & 68 & 88 & 118 & 17 \\
\hline $\mathrm{I} 30 \mathrm{~V}$ & 13170 & 93 & 93 & 38 & 56 & 72 & 118 & 20 \\
\hline I31V & 13170 & 93 & 93 & 38 & 56 & 72 & 118 & 20 \\
\hline $\mathrm{J} 30 \mathrm{~V}$ & 4677 & 87 & 140 & 62 & 78 & 99 & 120 & 17 \\
\hline J31V & 4677 & 87 & 140 & 62 & 78 & 99 & 120 & 17 \\
\hline $\mathrm{J} 32 \mathrm{~V}$ & 4677 & 87 & 140 & 62 & 78 & 99 & 120 & 17 \\
\hline K30V & 30720 & 36 & 468 & 24 & 44 & 58 & 118 & 17 \\
\hline K31V & 3964 & 305 & 3782 & 3 & 50 & 90 & 118 & 17 \\
\hline K32V & 5590 & 167 & 1115 & 41 & 54 & 73 & 118 & 17 \\
\hline K33V & 3388 & 290 & 3728 & 39 & 60 & 92 & 118 & 17 \\
\hline L30V & 11206 & 49 & 50 & 11 & 34 & 67 & 116 & 18 \\
\hline M30V & 14850 & 83 & 89 & 43 & 50 & 61 & 102 & 15 \\
\hline M31V & 14850 & 83 & 89 & 43 & 50 & 61 & 102 & 15 \\
\hline M32V & 14850 & 83 & 89 & 43 & 50 & 61 & 102 & 15 \\
\hline N30V & 8977 & 103 & 122 & 46 & 59 & 77 & 117 & 18 \\
\hline N31V & 8977 & 103 & 122 & 46 & 59 & 77 & 117 & 18 \\
\hline N32V & 8977 & 103 & 122 & 46 & 59 & 77 & 117 & 18 \\
\hline N33V & 8977 & 103 & 122 & 46 & 59 & 77 & 117 & 18 \\
\hline Q30V & 17224 & 48 & 52 & 41 & 50 & 58 & 115 & 18 \\
\hline Q31V & 17224 & 48 & 52 & 41 & 50 & 58 & 115 & 18 \\
\hline R30V & 12198 & 3 & 116 & 62 & 67 & 73 & 116 & 17 \\
\hline
\end{tabular}

Table 12: For DATASET J: PASSENGER LOADING BRIDGE and each video processing algorithm the table shows: a) the number of reported tracks; $b$ ) the mean number of frames reported within those tracks; $c$ ) the mean extent (first minus last frames indices plus one); d) the mean over all tracks of of the minimum interocular distance (IOD) reported; e) the mean of the mean IOD; $f$ ) the mean of the maximum IOD; g) the enrollment still image mean IOD; and $h)$ its standard deviation. For some algorithms (F, J, L, R) the tracks don't include all consecutive frames, so the extent of the track can exceed the number of frames in it. For the $D, F$, and $L$ algorithms, the reported still-image eye coordinates are erroneous.

\begin{tabular}{|c|c|c|c|c|c|c|c|c|c|}
\hline \multicolumn{4}{|c|}{ PARTICIPANT KEY } & SET & SCENE & CAMERA & SET & SCENE & CAMERA \\
\hline $\mathrm{A}=$ DIGITAL BARRIERS & $\mathrm{E}=$ NEUROTECHNOLOGY & $\mathrm{I}=\mathrm{EYEDEA}$ & $M=N E C$ & C & PHOTOJOURNALISM & PRO & $\mathrm{T}$ & CONCOURSE & PRO \\
\hline $\mathrm{B}=\mathrm{HBINNO}$ & $\mathrm{F}=$ VAPPLICA & $\mathrm{J}=\mathrm{HISIGN}$ & $\mathrm{N}=$ TOSHIBA & $\mathrm{J}$ & PASSENGER LOADING & PRO & $\mathrm{H}$ & CONCOURSE & PRO \\
\hline $\mathrm{C}=$ VIGILANT & $\mathrm{G}=\mathrm{MORPHO}$ & $\mathrm{K}=$ COGNITEC & $\mathrm{Q}=$ IMAGUS & $\mathrm{P}$ & SPORTS ARENA & CONSUMER & $\mathrm{U}$ & CHOKEPOINT & WEBCAM \\
\hline $\mathrm{D}=\mathrm{AYONIX}$ & $\mathrm{H}=3 \mathrm{M}$ COGENT & $\mathrm{L}=$ CYBEREXTRUDER & $\mathrm{R}=\mathrm{RANK}$ ONE & $\mathrm{L}$ & LUGGAGE RACK & WEBCAM & & & \\
\hline
\end{tabular}




\subsection{DATASET L: PASSENGER LUGGAGE}

Overview: This dataset is composed of video clips of 248 persons collected using two ceiling-mounted overhead cameras. The videos were collected to simulate use of inexpensive legacy cameras installed in a non-ideal location relative to the subjects who walk into the field of view, retrieve their luggage, and then proceed towards and underneath the cameras out of view - see Figure 26. The cameras are mounted at a height of about 2.13 meters ( 7 feet), above and to the left and right of the egress walkway. Each clip captures approximately 12-15 people walking toward the camera, and each person is typically in the field of view for 10-20 seconds. Key imaging properties for this dataset are summarized in Table

This dataset is challenging for recognition, because resolution is low and, particularly, pose is poor - subjects' head orientations are far from the optical axes. These problems exist in many legacy operational settings, such as shops and banks, that were never designed to support face recognition. Such data is nevertheless frequently used in investigations, because it is sometimes is the only evidence available to generate a lead.

The enrollment images are from DATASET U: PASSEN-

\begin{tabular}{|l|l|}
\hline Property & Value \\
\hline Camera & Logitech C920 \\
\hline Camera mounting & Ceiling mounted, to left and right of subject motion \\
\hline Camera height & 2.1 meters \\
\hline Range to subject & {$[0.7,5]$ meters } \\
\hline Frame rate & $15 \mathrm{sec}^{-1}$ or $30 \mathrm{sec}^{-1}$ \\
\hline Width & 1080 \\
\hline Height & 1920 \\
\hline Chroma sampling & YUV420 \\
\hline Nominal bitrate & $130000 \mathrm{~kb} \mathrm{sec}^{-1}$ \\
\hline Codec & WVC1 (advanced) 0x31435657 \\
\hline
\end{tabular}
GER GATE and embedded into a set of background

Table 13: Key imaging properties for DATASET L: PASSENGER LUGGAGE photographs such that the enrolled population size reaches $N=\{480,4800\}$, with exactly one image per person. All images are high quality frontal portraits in approximate conformance to the ISO full frontal image type - see Figure 8.

Experimental Design: The videos contain footage of subjects collecting hand luggage and then walking toward and underneath two ceiling mounted cameras. Mated scores are computed by searching 34 long video clips against an enrolled dataset of still face images of subjects known to be in the search videos. Nonmated scores are collected by comparing the same 34 video clips against the global nonmated enrollment dataset.

\begin{tabular}{|l|l|}
\hline Quantity & Value or description \\
\hline Mode & Video search to still enrollment \\
\hline Number of actors & 248 \\
\hline Number of non-actors & 0 \\
\hline Number of cameras & 2 (Left and right of field of view) \\
\hline Video duration with actors & 47.8 minutes \\
\hline Video duration no actors & 0 \\
\hline Subject motion & Usually single file toward and below the camera. Walking or stopping to pick up luggage. \\
\hline Number of clips & 34 \\
\hline Clip duration (frames) & Median 1611; Min 997; Max 3777 \\
\hline Number of enrolled subjects & 480,4800 \\
\hline Number of enrolled stills & 1 per subject \\
\hline Properties of enrolled stills & Frontal, close ICAO compliance; Mean IOD 106 pixels \\
\hline FNIR estimation & Actors present video vs. enrolled gallery \\
\hline FPIR estimation & Actors present video vs. separate non-actor gallery \\
\hline Candidate list length & 20 \\
\hline Number of persons in FOV & {$[4,7]$ usually } \\
\hline Video ground truth & Style A: See Figure 6 \\
\hline
\end{tabular}

Table 14: Key experimental design for the DATASET L: PASSENGER LUGGAGE results.

\begin{tabular}{|l|l|l|l|}
\hline \multicolumn{4}{|c|}{ PARTICIPANT KEY } \\
\hline $\mathrm{A}=$ DIGITAL BARRIERS & $\mathrm{E}=$ NEUROTECHNOLOGY & $\mathrm{I}=$ EYEDEA & $\mathrm{M}=$ NEC \\
\hline $\mathrm{B}=$ HBINNO & $\mathrm{F}=$ VAPPLICA & $\mathrm{J}=$ HISIGN & $\mathrm{N}=$ TOSHIBA \\
\hline $\mathrm{C}=$ VIGILANT & $\mathrm{G}=$ MORPHO & $\mathrm{K}=$ COGNITEC & $\mathrm{Q}=$ IMAGUS \\
\hline $\mathrm{D}=$ AYONIX & $\mathrm{H}=$ 3M COGENT & $\mathrm{L}=$ CYBEREXTRUDER & $\mathrm{R}=$ RANK ONE \\
\hline
\end{tabular}

\begin{tabular}{|l|l|l|l|l|l|}
\hline SET & SCENE & CAMERA & SET & SCENE & CAMERA \\
\hline C & PHOTOJOURNALISM & PRO & T & CONCOURSE & PRO \\
\hline J & PASSENGER LOADING & PRO & H & CONCOURSE & PRO \\
\hline P & SPORTS ARENA & CONSUMER & U & CHOKEPOINT & WEBCAM \\
\hline L & LUGGAGE RACK & WEBCAM & & & \\
\hline
\end{tabular}


Key experimental design details are summarized in Table 14.

Results: Table 16 presents FNIR for two gallery sizes, $N=\{480,4800\}$, at two decision thresholds, corresponding to false positives counts of $\operatorname{NFP}(T)=\{1,10\}$, and at two ranks $R=\{1,10\}$. Given the camera installation is not representative of a face recognition deployment, the rankbased metrics are probably more important, as they better represent the error rates that a forensic investigator would have to accept in a law enforcement investigation.

The table shows generally high error rates, making this dataset the worst among those in this report. The cameras are installed such that there is a high downward view angle (pitch) and high side-to-side angle (yaw). Additionally the optical resolution of the camera is low: The algorithms report mean interocular distances of 32 pixels - see Table 15. Illumination also is nonuniform. The result is that even for a small gallery, $N=480$, the best high-threshold miss rate is $28 \%(\mathrm{M} 30 \mathrm{~V})$, and only two developers have algorithms capable of FNIR below 50\%. For investigations, this improves to $11 \%$ when we consider the rank 1 error rate (M30V), and then eight developers have algorithms capable of producing FNIR $(480,1,0)$ below $50 \%$. Given that law enforcement investigations are often willing to follow any lead these error rates are still well below $100 \%$ and therefore low enough that investigators would continue to use face recognition as an investigative tool.

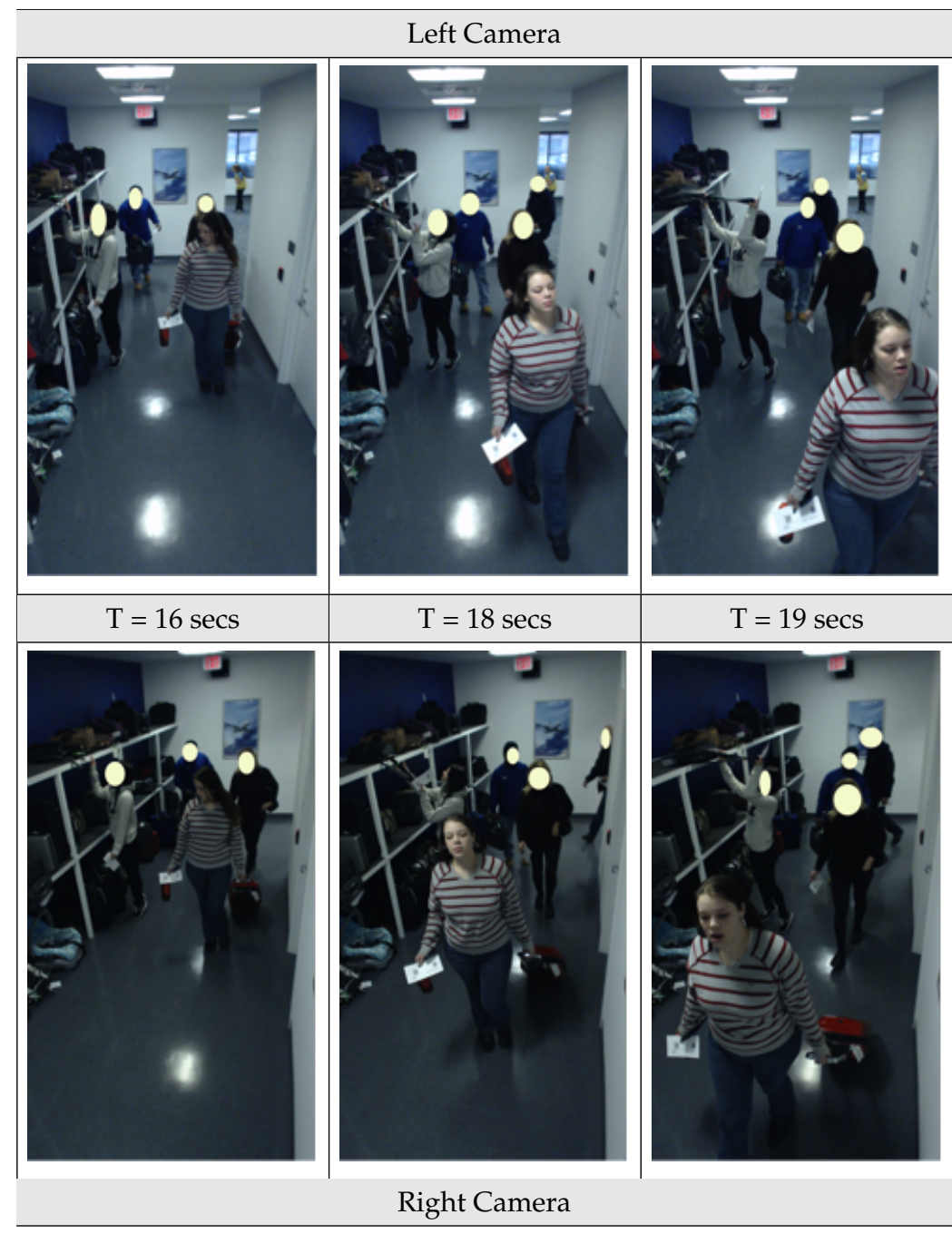

Figure 26: This example clip from DATASET L: PASSENGER LUGGAGE has the subject in view for around 20 seconds and contains 300 frames. Note the differences in illumination between the left and right camera positions. ${ }^{* *}$ The face images in this figure are from the DHS/ S\&T provided AEER dataset. The included subject consented to release their images in public reports. Subject 79195743 (Perm Granted). Where consent for public release from individuals in the background was not obtained, their faces were masked (yellow circles).

That said the enrolled population size here is only $N=480$. As $\mathrm{N}$ increases, low image quality is expected to cause rank one miss rates to increase as false positives displace some mates from rank one. This gives the usual decline in face recognition accuracy as more individuals are nominated to watch-lists. However in this dataset, the effect is larger. Thus, for the most accurate algorithm $(\mathrm{M} 30 \mathrm{~V}), \operatorname{FNIR}(4800, L, T)=0.53$ is almost double $\operatorname{FNIR}(480, L, T)=0.28$ for fixed T. For the best algorithm from the next most accurate developer on this set (G32V), error rates increase to 0.94 from 0.42 .

An interesting effect is that algorithms differ when a 10-fold increase in $\mathrm{N}$ is accompanied by a 10-fold increase in the number of tolerable false positives. Some algorithms (G30V, J31V, N3xV, R30V) conserve FNIR, as is expected from binomial models; some give moderately $(\mathrm{M} 3 \mathrm{xV}, \mathrm{J} 30 \mathrm{~V}, \mathrm{~J} 32 \mathrm{~V}, \mathrm{~N} 3 \mathrm{xV})$ or much $(\mathrm{G} 32 \mathrm{~V})$ worse FNIR; and still others improve

\begin{tabular}{|l|l|l|l|}
\hline \multicolumn{4}{|c|}{ PARTICIPANT KEY } \\
\hline $\mathrm{A}=$ DIGITAL BARRIERS & E = NEUROTECHNOLOGY & I = EYEDEA & $\mathrm{M}=$ NEC \\
\hline $\mathrm{B}=$ HBINNO & $\mathrm{F}=$ VAPPLICA & $\mathrm{J}=$ HISIGN & $\mathrm{N}=$ TOSHIBA \\
\hline $\mathrm{C}=$ VIGILANT & $\mathrm{G}=$ MORPHO & $\mathrm{K}=$ COGNITEC & $\mathrm{Q}=$ IMAGUS \\
\hline $\mathrm{D}=$ AYONIX & $\mathrm{H}=$ 3M COGENT & L = CYBEREXTRUDER & $\mathrm{R}=$ RANK ONE \\
\hline
\end{tabular}

\begin{tabular}{|l|l|l|l|l|l|}
\hline SET & SCENE & CAMERA & SET & SCENE & CAMERA \\
\hline C & PHOTOJOURNALISM & PRO & T & CONCOURSE & PRO \\
\hline J & PASSENGER LOADING & PRO & H & CONCOURSE & PRO \\
\hline P & SPORTS ARENA & CONSUMER & U & CHOKEPOINT & WEBCAM \\
\hline L & LUGGAGE RACK & WEBCAM & & & \\
\hline
\end{tabular}


$(\mathrm{H} 3 \mathrm{xV}, \mathrm{I} 3 \mathrm{xV}, \mathrm{N} 32 \mathrm{~V})$. The conclusion is that a system operator cannot rely on an assertion that gallery size increases can be offset by proportional increases of human-reviewed false positives. This is addressed later, in the discussion of Figure 36 for Dataset $\mathrm{H}$.

\begin{tabular}{|c|c|c|c|c|c|c|c|c|}
\hline DATASET L & \multicolumn{6}{|c|}{ VIDEO SEARCH } & \multirow{2}{*}{\multicolumn{2}{|c|}{$\begin{array}{l}\text { STILL IMAGE } \\
\text { IOD (PIXELS) }\end{array}$}} \\
\hline & NUMBER OF & MEAN TRACK & MEAN TRACK & MEAN & MEAN & MEAN & & \\
\hline ALGORITHM & TRACKS & LENGTH (FRAMES) & EXTENT (FRAMES) & MIN IOD (PX) & MEAN IOD (PX) & MAX IOD (PX) & MEAN & STD. DEV \\
\hline $\mathrm{A} 30 \mathrm{~V}$ & 4071 & 7 & 7 & 31 & 33 & 36 & 116 & 20 \\
\hline A31V & 4071 & 7 & 7 & 31 & 33 & 36 & 116 & 20 \\
\hline B30V & 764 & 34 & 42 & 28 & 48 & 185 & 118 & 21 \\
\hline C30V & 2062 & 5 & 6 & 39 & 42 & 46 & 119 & 21 \\
\hline C31V & 2482 & 5 & 5 & 35 & 38 & 42 & 119 & 21 \\
\hline D30V & 3794 & 3 & 3 & 58 & 60 & 61 & 118 & 22 \\
\hline D31V & 887 & 22 & 23 & 37 & 46 & 58 & 118 & 22 \\
\hline E30V & 664 & 41 & 53 & 27 & 37 & 56 & 117 & 20 \\
\hline E31V & 664 & 41 & 53 & 27 & 37 & 56 & 117 & 20 \\
\hline F30V & 4213 & 3 & 5 & 35 & 36 & 38 & 116 & 20 \\
\hline G30V & 463 & 13 & 31 & 35 & 43 & 57 & 118 & 21 \\
\hline G31V & 678 & 27 & 87 & 32 & 41 & 54 & 117 & 21 \\
\hline G32V & 678 & 27 & 87 & 32 & 41 & 54 & 117 & 21 \\
\hline H30V & 714 & 29 & 31 & 35 & 45 & 62 & 119 & 21 \\
\hline H31V & 714 & 29 & 31 & 35 & 45 & 62 & 119 & 21 \\
\hline H32V & 714 & 29 & 31 & 35 & 45 & 62 & 119 & 21 \\
\hline I30V & 1218 & 39 & 39 & 12 & 23 & 34 & 120 & 21 \\
\hline I31V & 1218 & 39 & 39 & 12 & 23 & 34 & 120 & 21 \\
\hline $\mathrm{J} 30 \mathrm{~V}$ & 445 & 19 & 24 & 44 & 54 & 68 & 121 & 21 \\
\hline $\mathrm{J} 31 \mathrm{~V}$ & 445 & 19 & 24 & 44 & 54 & 68 & 121 & 21 \\
\hline $\mathrm{J} 32 \mathrm{~V}$ & 445 & 19 & 24 & 44 & 54 & 68 & 121 & 21 \\
\hline K30V & 4093 & 6 & 49 & 16 & 21 & 25 & 119 & 21 \\
\hline K31V & 440 & 81 & 967 & 8 & 29 & 63 & 119 & 21 \\
\hline K32V & 562 & 25 & 65 & 31 & 37 & 46 & 119 & 21 \\
\hline K33V & 310 & 50 & 325 & 31 & 41 & 58 & 119 & 21 \\
\hline L30V & 463 & 15 & 16 & 9 & 32 & 53 & 116 & 20 \\
\hline M30V & 1094 & 29 & 33 & 26 & 32 & 42 & 104 & 19 \\
\hline M31V & 1094 & 29 & 33 & 26 & 32 & 42 & 104 & 19 \\
\hline M32V & 1094 & 29 & 33 & 26 & 32 & 42 & 104 & 19 \\
\hline N30V & 598 & 35 & 46 & 26 & 39 & 58 & 118 & 21 \\
\hline N31V & 598 & 35 & 46 & 26 & 39 & 58 & 118 & 21 \\
\hline N32V & 598 & 35 & 46 & 26 & 39 & 58 & 118 & 21 \\
\hline N33V & 598 & 35 & 46 & 26 & 39 & 58 & 118 & 21 \\
\hline Q30V & 1242 & 24 & 26 & 25 & 32 & 40 & 116 & 20 \\
\hline Q31V & 1242 & 24 & 26 & 25 & 32 & 40 & 116 & 20 \\
\hline R30V & 1148 & 2 & 51 & 50 & 53 & 57 & 116 & 20 \\
\hline
\end{tabular}

Table 15: For DATASET L: PASSENGER LUGGAGE and each video processing algorithm the table shows: a) the number of reported tracks; $b$ ) the mean number of frames reported within those tracks; c) the mean extent (first minus last frames indices plus one); $d$ ) the mean over all tracks of of the minimum interocular distance (IOD) reported; e) the mean of the mean IOD; $f$ ) the mean of the maximum IOD; g) the enrollment still image mean IOD; and h) its standard deviation. For some algorithms (F, J, L, R) the tracks don't include all consecutive frames, so the extent of the track can exceed the number of frames in it. For the $D, F$, and $L$ algorithms, the reported still-image eye coordinates are erroneous.

\begin{tabular}{|l|l|l|l|}
\hline \multicolumn{4}{|c|}{ PARTICIPANT KEY } \\
\hline A = DIGITAL BARRIERS & E = NEUROTECHNOLOGY & I = EYEDEA & $\mathrm{M}=$ NEC \\
\hline B = HBINNO & F = VAPPLICA & J = HISIGN & = TOSHIBA \\
\hline C = VIGILANT & G = MORPHO & K = COGNITEC & Q = IMAGUS \\
\hline D = AYONIX & H = 3M COGENT & L = CYBEREXTRUDER & R = RANK ONE \\
\hline
\end{tabular}

\begin{tabular}{|l|l|l|l|l|l|}
\hline SET & SCENE & CAMERA & SET & SCENE & CAMERA \\
\hline C & PHOTOJOURNALISM & PRO & T & CONCOURSE & PRO \\
\hline J & PASSENGER LOADING & PRO & H & CONCOURSE & PRO \\
\hline P & SPORTS ARENA & CONSUMER & U & CHOKEPOINT & WEBCAM \\
\hline L & LUGGAGE RACK & WEBCAM & & & \\
\hline
\end{tabular}




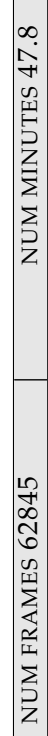

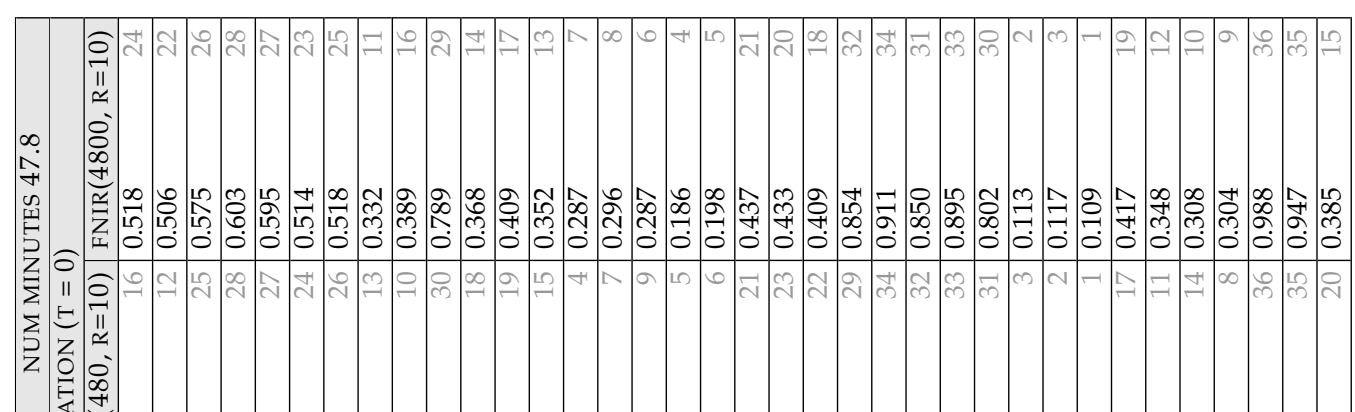

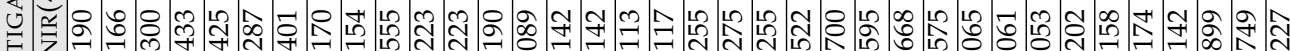

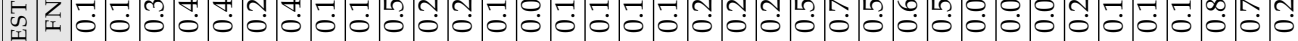

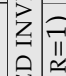

10

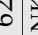

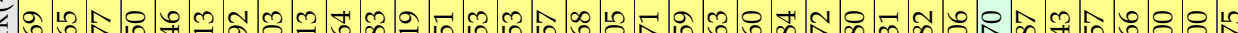

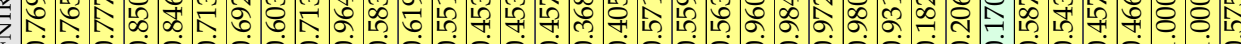

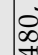

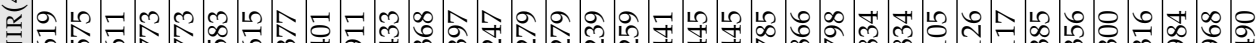

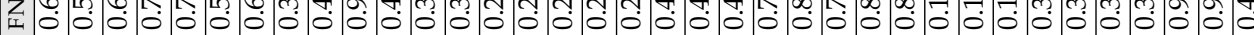

○ III

E

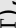

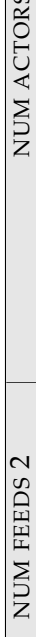

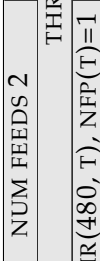

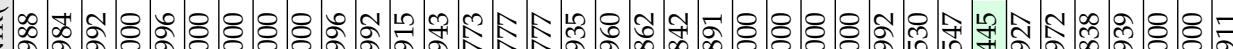
$z$ O. II

孛

它

+

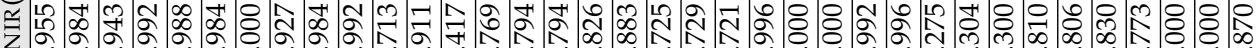

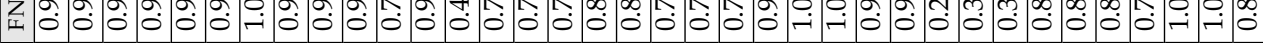

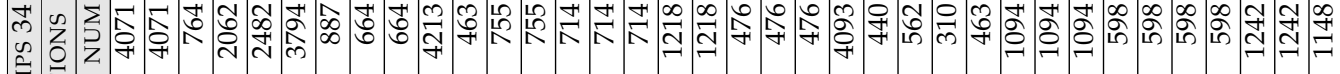

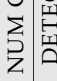

0
0
0

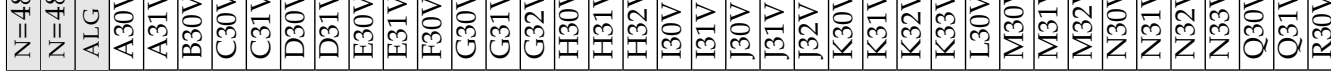

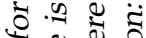

$\widehat{E} \cong$

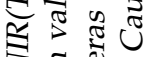

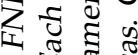

ช

हี उ

ปี

ปี -15

范

픈 3

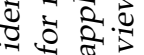

वै $\approx$

क ฐ

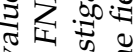

¿ 8

$\Xi$ ฐ $ฐ$

जิ

के ठี

ส ㄷำ

ธี่

ส $\vec{\Xi}$ ป

줘

उ สี

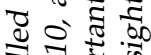

당져

ฮิ

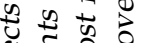

ปิ छิ

के 0

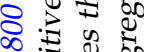

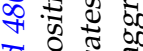

₹

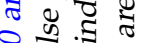

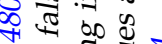

노연

3 bo

ธี ซี

I 1 ज

สี क्ष

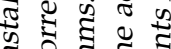

ฐ 8 ₹

당

गิ สิ

क्⿹ స

ฐ ฐ

अี

乙

जิ

๘ สี

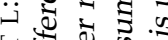

प्य

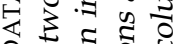

ब ส

ฐ 2 它

t)

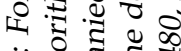

$\because \frac{0}{\pi}$ है हा

㓂

\begin{tabular}{|c|c|c|c|c|c|c|c|c|c|}
\hline \multicolumn{4}{|c|}{ PARTICIPANT KEY } & SET & SCENE & CAMERA & SET & SCENE & CAMERA \\
\hline $\mathrm{C}=$ VIGILANT & $\mathrm{G}=\mathrm{MORPHO}$ & $\mathrm{K}=$ COGNITEC & $Q=$ IMAGUS & $\mathrm{P}$ & SPORTS ARENA & CONSUMER & $\mathrm{U}$ & CHOKEPOINT & WEBCAM \\
\hline $\mathrm{D}=\mathrm{AYONIX}$ & $\mathrm{H}=3 \mathrm{M}$ COGENT & $\mathrm{L}=$ CYBEREXTRUDER & $\mathrm{R}=$ RANK ONE & $\mathrm{L}$ & LUGGAGE RACK & WEBCAM & & & \\
\hline
\end{tabular}




\subsection{DATASET P: SPORTS ARENA}

Overview: This dataset is composed of videos from eleven cameras mounted in the indoor access areas surrounding a sports arena. Video was collected on five evenings corresponding to real sporting events. The collection includes some known actors and many more incidental unknown persons. The cameras collected imagery for several hours each evening, with sunset occuring early in the evenings.

The cameras are visible to the subjects. Therefore, the subjects are in-principle aware of the cameras, but they largely ignore them. The number of video clips is very large, comprised of clips known to contain actors, and clips known not to contain actors. In all cases, there are multiple faces visible in the clips. The duration of the subjects' appearance is generally 10 to 30 seconds, but there are instances where a subject is only visible for a much briefer interval. Key imaging properties for this dataset are summarized in Table 17.

Enrolled still images: Images were collected under controlled lighting, background, and pose conditions. The FIVE API [20] supports multiple still image input along with nominal pose (yaw and pitch)

\begin{tabular}{|l|l|}
\hline Property & Value \\
\hline Cameras & Canon VIXIA HF R400 \\
\hline Camera mounting & Fixed to wall or door, no attractor \\
\hline Camera ht. hallway & 1.83 and 2.44 meters \\
\hline Camera ht. door & 2.44 meters \\
\hline Range to subject & {$[1,10]$ meters } \\
\hline Frame rate & $24 \mathrm{sec}^{-1}$ \\
\hline Width & 1920 \\
\hline Height & 1080 \\
\hline Chroma sampling & YUV420 $^{-1}$ \\
\hline Nominal bitrate & $24 \mathrm{Mbit} \mathrm{sec}^{-1}$ \\
\hline Codec & AVC H264 \\
\hline
\end{tabular}

Table 17: Key imaging properties for DATASET P: SPORTS ARENA

values to the algorithm software for template generation, which enables performance analysis when the algorithm is provided with multiple images/poses of the subject.

Full-frontal: This gallery is composed of $\mathrm{N}=480$ subjects, with exactly one full front image per subject.

Multi-pose: This gallery is composed of $\mathrm{N}=480$ subjects, with three images per subject as in Figure 33.

Videos: The DATASET P: SPORTS ARENA videos were collected from eleven cameras, in three groups:

Doors: Figure 28 shows example frames collected from three cameras mounted over three entry-exit doors. These are numbered 5,6 and 7. Here the subjects walk into the building toward the cameras at the beginning of the sporting event and, after reversing the cameras viewpoint, from subjects walking toward the cameras as they exit the venue. Table 36 in Appendix 1 include statistics for intercoular distances this group, as reported by each algorithm.

Queue: Figure 27 shows images collected from two groups of cameras. The first includes images from three cameras (labelled 2, 3,4) that are mounted in a concession stand queue where subjects walk mostly toward and transverse to the cameras. These cameras are designated as "near" in that subjects are close to the camera. Tables 39 and 40 show interocular distance statisics for, respectively, low and high mounted cameras, with the low cameras giving, on average, somewhat smaller detected faces.

Hallway: Figure 27 also shows examples from the second group of cameras. The first group is comprised of five cameras mounted at the end of a hallway that observe subjects walking both toward and away from the cameras. These cameras are numbered 1, 9, 10,11, 12 and designated as "far". The faces are typically far from the camera, although in some cases, particularly camera 1, the subjects are closer. The cameras are mounted at a height of 1.83 meters (6 feet, "low") or 2.44 meters ( 8 feet, "high"). Tables 37 and 38 include interocular distance statistics

\begin{tabular}{|l|l|l|l|}
\hline \multicolumn{5}{|c|}{ PARTICIPANT KEY } \\
\hline $\mathrm{A}=$ DIGITAL BARRIERS & $\mathrm{E}=$ NEUROTECHNOLOGY & $\mathrm{I}=$ EYEDEA & $\mathrm{M}=$ NEC \\
\hline $\mathrm{B}=$ HBINNO & $\mathrm{F}=$ VAPPLICA & $\mathrm{J}=$ HISIGN & $\mathrm{N}=$ TOSHIBA \\
\hline $\mathrm{C}=$ VIGILANT & $\mathrm{G}=$ MORPHO & $\mathrm{K}=$ COGNITEC & $\mathrm{Q}=$ IMAGUS \\
\hline $\mathrm{D}=$ AYONIX & $\mathrm{H}=$ 3M COGENT & $\mathrm{L}=$ CYBEREXTRUDER & $\mathrm{R}=$ RANK ONE \\
\hline
\end{tabular}

\begin{tabular}{|l|l|l|l|l|l|}
\hline SET & SCENE & CAMERA & SET & SCENE & CAMERA \\
\hline C & PHOTOJOURNALISM & PRO & T & CONCOURSE & PRO \\
\hline J & PASSENGER LOADING & PRO & H & CONCOURSE & PRO \\
\hline P & SPORTS ARENA & CONSUMER & U & CHOKEPOINT & WEBCAM \\
\hline L & LUGGAGE RACK & WEBCAM & & & \\
\hline
\end{tabular}




\begin{tabular}{|l|l|}
\hline Quantity & Value or description \\
\hline Mode & Video search to still enrollment \\
\hline Number of actors & 64 \\
\hline Number of non-actors & Many \\
\hline Number of cameras & 11 \\
\hline Video duration with actors & 7995 mins over four evenings \\
\hline Video duration no actors & 2883 mins over one different evening \\
\hline Number of clips actors & 16460 \\
\hline Number of clips no actors & 5809 \\
\hline Subject motion & Mostly toward, but many laterial or away from cameras \\
\hline Clip duration (frames) & Median 634; Min 24; Q25 369; Q75 774; Max 2881 \\
\hline Number of enrolled subjects & 480 \\
\hline Number of enrolled stills & 1 FF (also separately 1FF + 1QR + 1QL) per subject \\
\hline Properties of enrolled stills & Frontal, close ICAO compliance; Mean IOD 111 pixels \\
\hline FNIR estimation & Actors present video vs. enrolled gallery \\
\hline FPIR estimation & Actors absent video vs. same enrolled gallery \\
\hline Candidate list length & 20 \\
\hline Number of persons in FOV & {$[0,30]$ across FOV, none dominant } \\
\hline Video ground truth & Style A: See Figure 6 \\
\hline
\end{tabular}

Table 18: Key experimental design for the DATASET P: SPORTS ARENA results.

for, respectively, low and high mounted cameras. The reported interocular distances are significantly lower, on average, than in the queue.

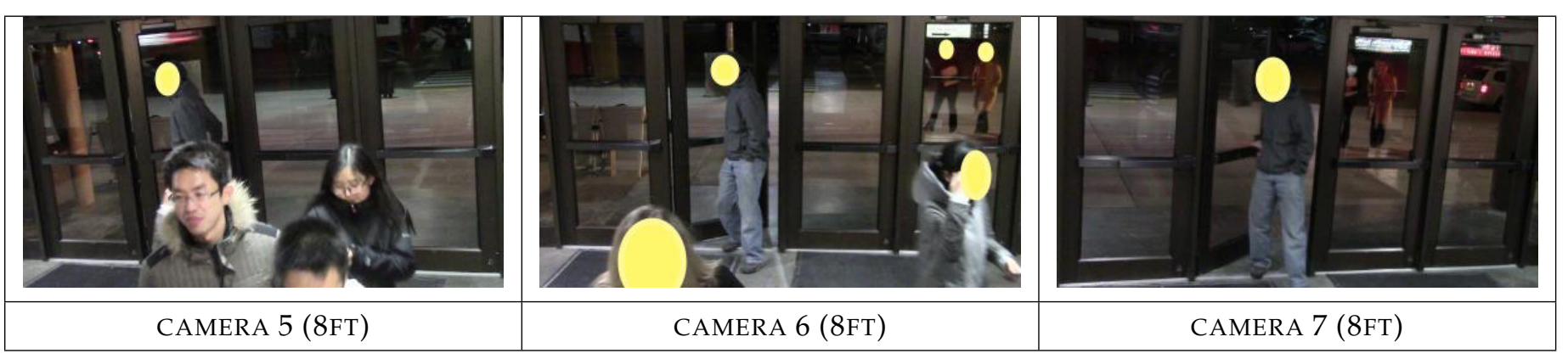

Figure 28: DATASET P: SPORTS ARENA : Examples from public-area surveillance video clips (entry and exit). **The face images in this figure are from a DHS / S\&T provided dataset. Written consent from DHS / S\&T to use these images in public reports was obtained. Actors AS, AY (Perm Granted). Lacking individual consent, faces are masked (yellow circle).

Experimental Design: The videos contain footage of people in various places inside a sports arena including the main entrance and exit doors, the hallway, and in the queue to a concession stand.

Mated scores are computed over 16460 video clips searched against an enrollment dataset of size 480 of still face images of subjects known to be in the search videos. The enrollment database is padded with FERET and mugshot images to attain the desired size.

Nonmated scores scores are computed by running many actors-absent video clips. This is done for each camera.

Key experimental design details are summarized in Table 18.

Detection Results: Table 19 includes total detection counts over 2883 minutes of video. As with other datasets, the algorithms vary widely in the number of reported face tracks. The most accurate algorithm M32V reports 144311 tracks from all cameras, corresponding to 54 detections per minute, on average. The G30V algorithm reports just 36630 tracks

\begin{tabular}{|l|l|l|l|}
\hline \multicolumn{4}{|c|}{ PARTICIPANT KEY } \\
\hline $\mathrm{A}=$ DIGITAL BARRIERS & E = NEUROTECHNOLOGY & I = EYEDEA & $\mathrm{M}=$ NEC \\
\hline $\mathrm{B}=$ HBINNO & $\mathrm{F}=$ VAPPLICA & $\mathrm{J}=$ HISIGN & $\mathrm{N}=$ TOSHIBA \\
\hline $\mathrm{C}=$ VIGILANT & $\mathrm{G}=$ MORPHO & $\mathrm{K}=$ COGNITEC & $\mathrm{Q}=$ IMAGUS \\
\hline $\mathrm{D}=$ AYONIX & $\mathrm{H}=$ 3M COGENT & L = CYBEREXTRUDER & $\mathrm{R}=$ RANK ONE \\
\hline
\end{tabular}

\begin{tabular}{|l|l|l|l|l|l|}
\hline SET & SCENE & CAMERA & SET & SCENE & CAMERA \\
\hline C & PHOTOJOURNALISM & PRO & T & CONCOURSE & PRO \\
\hline J & PASSENGER LOADING & PRO & H & CONCOURSE & PRO \\
\hline P & SPORTS ARENA & CONSUMER & U & CHOKEPOINT & WEBCAM \\
\hline L & LUGGAGE RACK & WEBCAM & & & \\
\hline
\end{tabular}




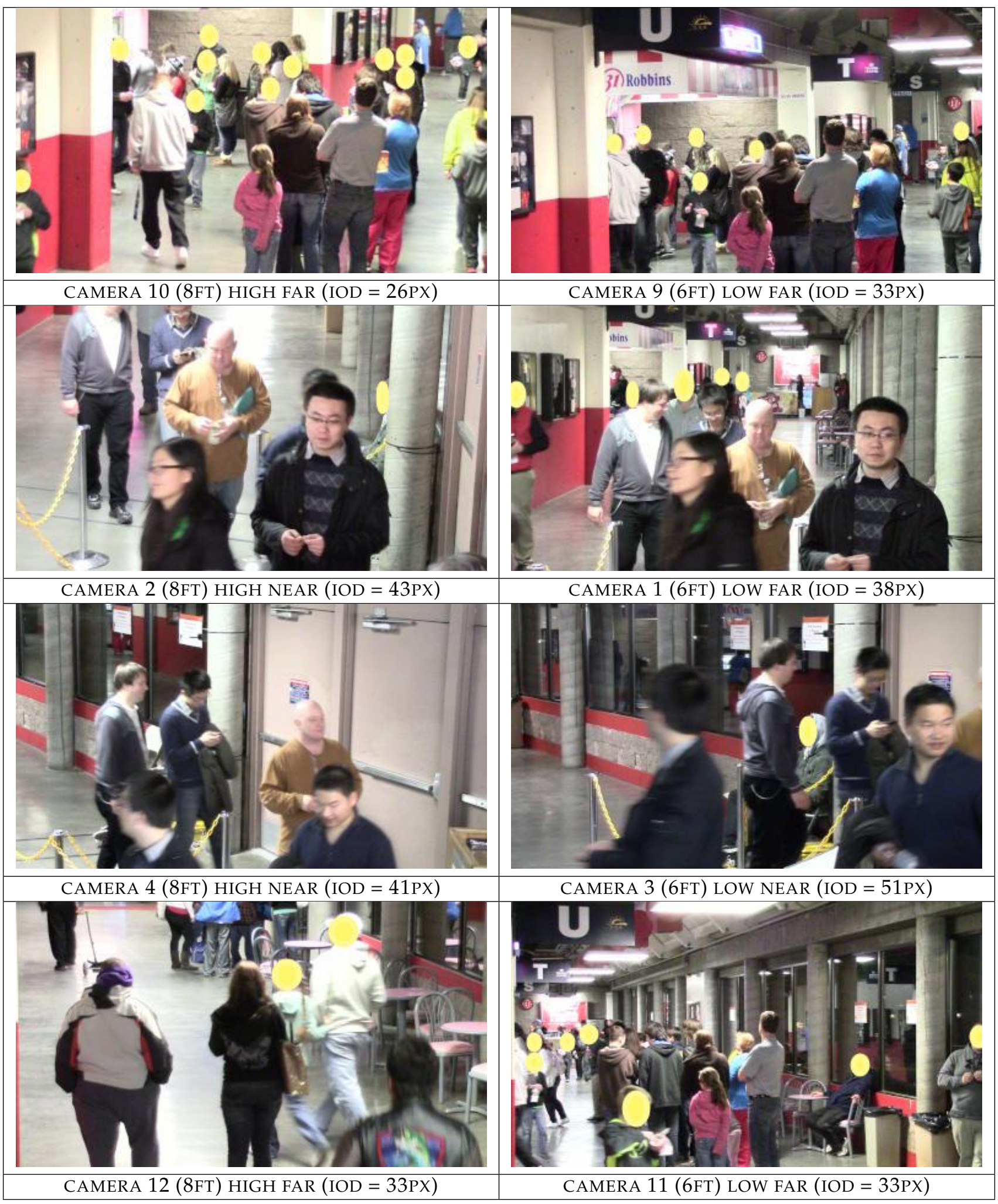

Figure 27: DATASET P: SPORTS ARENA : Examples from public-area surveillance video clips (hallway and queue). Results are grouped by the labels low-high near-far. For camera 1 the FAR label is imperfect in that subjects are aquired both near and far, and accuracy is more inline with far acquisition. The interocular distances (IOD) values are global averages from four algorithms over all reported tracks; peak IOD values will be larger. See tables in the Appendix. The left and right images are contemporaneous (except in the last row). ${ }^{* *}$ The face images in this figure are from a DHS/ S\&T provided dataset. Written consent from DHS / S\&T to use these images in public reports was obtained on August 12, 2016. Actors AS, AV, AW, AY, I, V (Perm Granted). Lacking individual consent, faces are masked (yellow circle).

\begin{tabular}{|c|c|c|c|c|c|c|c|c|c|}
\hline \multicolumn{4}{|c|}{ PARTICIPANT KEY } & \multirow{2}{*}{\begin{tabular}{|l|} 
SET \\
C \\
\end{tabular}} & SCENE & \multirow{2}{*}{$\begin{array}{l}\text { CAMERA } \\
\text { PRO }\end{array}$} & \multirow{2}{*}{\begin{tabular}{|l|} 
SET \\
$T$
\end{tabular}} & \multirow{2}{*}{\begin{tabular}{|l|} 
SCENE \\
CONCOURSE
\end{tabular}} & \multirow{2}{*}{\begin{tabular}{|l|} 
CAMERA \\
PRO
\end{tabular}} \\
\hline $\mathrm{A}=$ DIGITAL BARRIERS & $\mathrm{E}=$ NEUROTECHNOLOGY & $\mathrm{I}=$ EYEDEA & $M=N E C$ & & PHOTOJOURNALISM & & & & \\
\hline $\mathrm{C}=$ VIGILANT & $\mathrm{G}=\mathrm{MORPHO}$ & $\mathrm{K}=$ COGNITEC & $Q=$ IMAGUS & $\mathrm{P}$ & SPORTS ARENA & CONSUMER & $\mathrm{U}$ & CHOKEPOINT & WEBCAM \\
\hline $\mathrm{D}=\mathrm{AYONIX}$ & $\mathrm{H}=3 \mathrm{M}$ COGENT & $\mathrm{L}=$ CYBEREXTRUDER & $\mathrm{R}=\mathrm{RANK}$ ONE & $\mathrm{L}$ & LUGGAGE RACK & WEBCAM & & & \\
\hline
\end{tabular}


$\left(14 \mathrm{~min}^{-1}\right)$, F30V gives $386103\left(148 \mathrm{~min}^{-1}\right)$. This variability arises because detection and tracking is a non-trivial task, and is a tunable parameter. For example, the $\mathrm{K} 3 \mathrm{xV}$ algorithms have a 20-fold spread in the number of tracks reported. A verbose detector is only less accurate if its detected faces yield high-scoring false positive candidates.

A parsimonious detector is less accurate only if it misses faces (of actors). In any case, the hardware cycles needed to execute searches rise linearly with detection rates.

Figure 30 plots detection counts alongside false positive counts. It does this for the five camera groups. It uses a log scale because, while the number of detections is in the tens of thousands, we set the threshold to produce just 800 false positives. This was achieved by setting the threshold globally, over all impostor video clips. Operationally, it would be more advisable to set the threshold on a per camera or per location basis. The reason for this is because false positives vary by location, or more specifically, with the imaging environment. Notably high mounted cameras give fewer false positives, expecially when close to the subject. The most false positives are produced in the LOW-FAR camera configuration where detection rates are also highest.

Recognition Results: Detection and accuracy results are tabulated in Table 19. The dotplots of Figure 29 are included to visualize both identifcation-mode and investigation-mode miss rates i.e. FNIR $(480, L, T)$ and $\operatorname{FNIR}(480,1,0)$. In the former case the decision threshold, T, is set to correspond to 200 false positives over all tracks detected in the 2883 minutes of video footage that does not contain actors (see Table 18). The number 200 may imply a significant level of human adjudication but it corresponds to one false positive for every 11.7 minutes of video footage on average. Thus the decision threshold is more stringent than that used in the DATASET U: PASSENGER GATE analysis because the footage is much longer and contains many more people, on average.

Overall accuracy: Given the challenging nature of the video in terms of illumination and the use of an inexpensive imaging system, the identification miss rates are generally higher than with other datasets.

Door cameras: As shown in Table 19, the FNIR values at the door entry and exit are much worse than for the hallway mounted cameras. The most accurate algorithm fails to place the actor at rank 1 fully $40 \%$ of the time. When the threshold is raised, the best value ascends to $64 \%$. This is due to the high elevation (pitch) angle, adverse lighting - see Figure 28 - and the short duration that a subject is in view, corresponding to a high radial component of subject motion. In the surveillance mode when threshold is set to limit false positives, only one manufacturer is capable of missing fewer than $80 \%$ of the subjects. This essentially says that face recognition is not viable without deliberate tested improvements to this imaging environment.

Queue cameras: The best FNIR values occur for subjects in the queue. There, rank 1 recognition miss rates are below $6 \%$, and when the threshold is raised, remain below 13\% (algorithm M32V). Two factors are at play. First is resolution, as discussed in the next bullet. Second is duration of imaging: As subjects approaching the queue cameras come to a standstill, their track lengths (durations) are longer, affording more viewss of the face. Tracking then is helped by increased resolution.

Hallway cameras: FNIR values are markedly worse for subjects imaged at greater distances along the hallway. This is especially true for identification-mode high-threshold FNIR estimates, where FNIR is often double the value for the queue. Recognition accuracy is likely driven by resolution: From the eye coordinates reported by the algorithms - see Appendix 1 - it is clear that the near view interocular distances are almost double those of faces in the far field hallway cameras.

\begin{tabular}{|l|l|l|l|}
\hline \multicolumn{4}{|c|}{ PARTICIPANT KEY } \\
\hline $\mathrm{A}=$ DIGITAL BARRIERS & E = NEUROTECHNOLOGY & I = EYEDEA & $\mathrm{M}=$ NEC \\
\hline $\mathrm{B}=$ HBINNO & $\mathrm{F}=$ VAPPLICA & $\mathrm{J}=$ HISIGN & $\mathrm{N}=$ TOSHIBA \\
\hline $\mathrm{C}=$ VIGILANT & $\mathrm{G}=$ MORPHO & $\mathrm{K}=$ COGNITEC & $\mathrm{Q}=$ IMAGUS \\
\hline $\mathrm{D}=$ AYONIX & $\mathrm{H}=$ 3M COGENT & L = CYBEREXTRUDER & $\mathrm{R}=$ RANK ONE \\
\hline
\end{tabular}

\begin{tabular}{|l|l|l|l|l|l|}
\hline SET & SCENE & CAMERA & SET & SCENE & CAMERA \\
\hline C & PHOTOJOURNALISM & PRO & T & CONCOURSE & PRO \\
\hline J & PASSENGER LOADING & PRO & H & CONCOURSE & PRO \\
\hline P & SPORTS ARENA & CONSUMER & U & CHOKEPOINT & WEBCAM \\
\hline L & LUGGAGE RACK & WEBCAM & & & \\
\hline
\end{tabular}


Camera height: Cameras mounted at 2.44 meters give worse miss rates than those mounted lower, at 1.83 meters. This effect is more pronounced when imaging subjects near to the camera - as the look down angles are larger and at higher recognition thresholds. The effect is highly algorithm dependent, with the I and M having more immunity to pitch angle.

\subsubsection{Effect of fusing results over cameras}

This report mostly addresses the question of how well algorithms can identify an individual in a video clip from a single camera, and the accuracy figures are very useful for comparing algorithms. In settings where several cameras are used to observe a volume, accuracy can be improved - this was addressed in section 5.3 - essentially by spatially fusing recognition outcomes from a single appearance of a subject. Here we address fusion over time, answering the question of how well persons are recognized at any point during an event, in this case over an evening. Our study is not well controlled in that the actors were not instructed to appear in a location a fixed number of times during the event. Instead we have imbalance where the number of appearances varies across subjects.

The results of Figure 31 plot identification error rates with fusion against those without. Fusion is implemented by taking the highest scoring candidate entry over all sightings of a subject by all cameras in camera group over the course of an event. Referencing Figures 27 and 28 and the camera group definitions (door cameras 5-7, high mounted far cameras 10,12 , high near 2,4, low mounted far 9, 11, 1, and near 3. Camera 1 was installed as a near-field camera but is grouped with the other far cameras because algorithms acquire many far faces and give worse accuracy accordingly. Fusion substantially improves accuracy values, often FNIR is reduced by factors of three or more. This is directly related to the number of appearances of a subject and would be mostly ineffective for applications where a subject passes a single camera exactly once. This is evident for the door group - fusion is less effective simply because subjects usually only appeared in that vicinity twice, at the beginning and end of an evening event. The technique also has reduced operational relevance, because the fusion is necessarily "after action" meaning it is no longer a real-time operation. The technique may be useful for applications that seek to determine whether an individual appeared at any time. For example, did aircraft maintenance staff board the aircraft at any point during the three hour stopover.

Figure 32 takes the fusion further, by fusing over all cameras in addition to all appearances. The result is that the most accurate algorithm correctly identify all the actors present in the dataset. This generally supports the conclusion that more cameras support better identification rates. While effective, it's efficiency in terms of cost and time, is questionable. Instead of equipping a building with many cameras, it is likely more worthwhile to construct a volume through which all subjects pass, and to install cameras and illumination there, paying attention to pose angles and optical specifications including resolution, depth of field, and field of view.

\subsubsection{Effect of enrolling multiple pose views}

To test whether algorithms are capable of exploiting multiple views of a face, we executed the same sets of genuine and impostor video searches against two galleries. In the first, subjects were enrolled with a single full frontal still image. In the second, subject were enrolled with three images, as shown in Figure 33. This is achieved via the FIVE API [20] which supports providing multiple still images with their nominal pose (yaw and pitch) values to the algorithm software in a single template generation function call. This allows the software to exploit the set of images in whatever manner it

\begin{tabular}{|l|l|l|l|}
\hline \multicolumn{5}{|c|}{ PARTICIPANT KEY } \\
\hline $\mathrm{A}=$ DIGITAL BARRIERS & $\mathrm{E}=$ NEUROTECHNOLOGY & $\mathrm{I}=$ EYEDEA & $\mathrm{M}=$ NEC \\
\hline $\mathrm{B}=$ HBINNO & $\mathrm{F}=$ VAPPLICA & $\mathrm{J}=$ HISIGN & $\mathrm{N}=$ TOSHIBA \\
\hline $\mathrm{C}=$ VIGILANT & $\mathrm{G}=$ MORPHO & $\mathrm{K}=$ COGNITEC & $\mathrm{Q}=$ IMAGUS \\
\hline $\mathrm{D}=$ AYONIX & $\mathrm{H}=$ 3M COGENT & $\mathrm{L}=$ CYBEREXTRUDER & $\mathrm{R}=$ RANK ONE \\
\hline
\end{tabular}

\begin{tabular}{|l|l|l|l|l|l|}
\hline SET & SCENE & CAMERA & SET & SCENE & CAMERA \\
\hline C & PHOTOJOURNALISM & PRO & T & CONCOURSE & PRO \\
\hline J & PASSENGER LOADING & PRO & H & CONCOURSE & PRO \\
\hline P & SPORTS ARENA & CONSUMER & U & CHOKEPOINT & WEBCAM \\
\hline L & LUGGAGE RACK & WEBCAM & & & \\
\hline
\end{tabular}




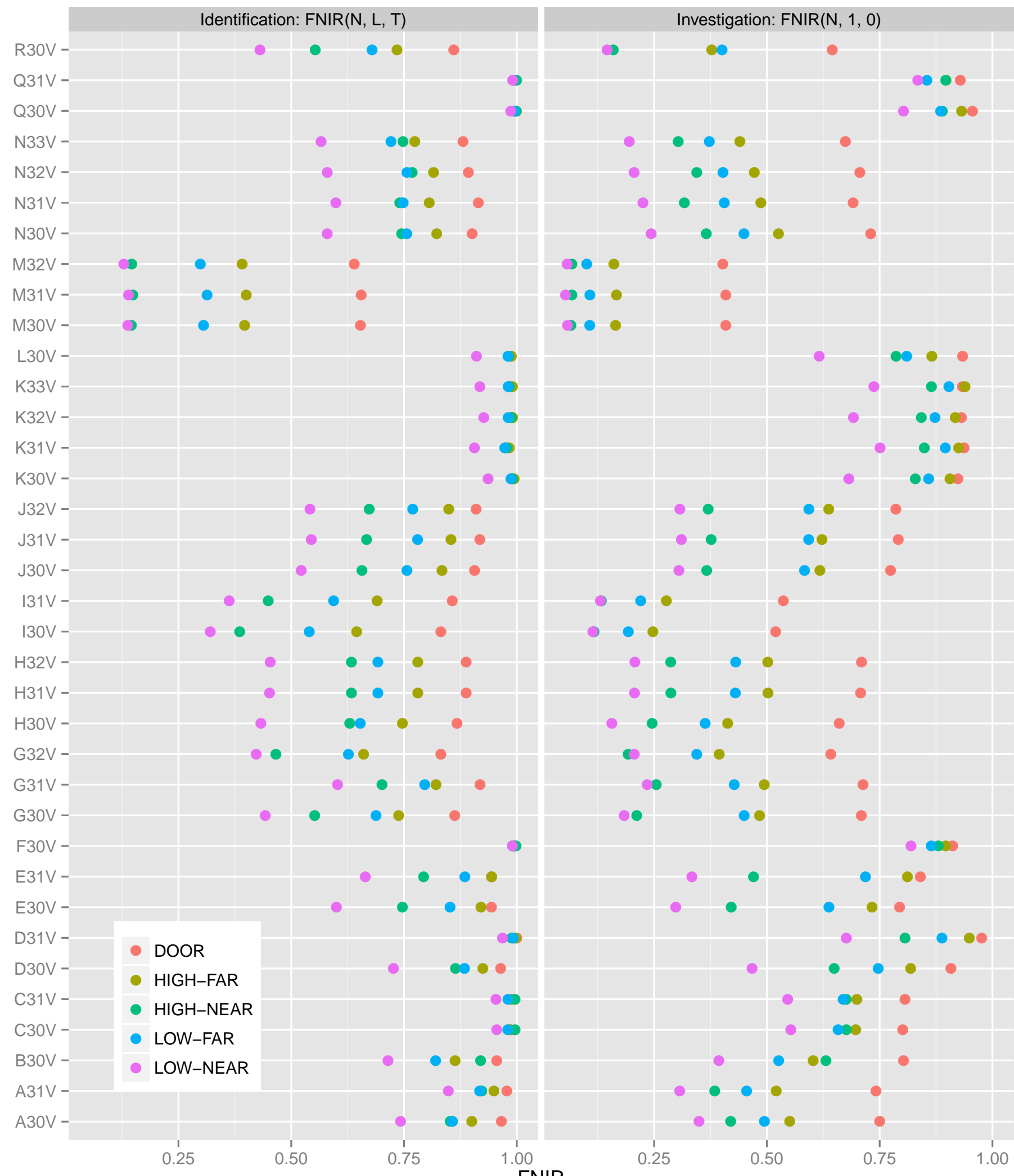

FNIR

Figure 29: In the left panel, threshold-based FNIRs for each algorithm over the DATASET P: SPORTS ARENA dataset with threshold set to yield NFP =20). At right, is the investigation mode, rank 1, miss rates. The colored dots indicate the camera mounting height and range.

\begin{tabular}{|l|l|l|l|}
\hline \multicolumn{4}{|c|}{ PARTICIPANT KEY } \\
\hline $\mathrm{A}=$ DIGITAL BARRIERS & $\mathrm{E}=$ NEUROTECHNOLOGY & $\mathrm{I}=$ EYEDEA & $\mathrm{M}=$ NEC \\
\hline $\mathrm{B}=$ HBINNO & $\mathrm{F}=$ VAPPLICA & $\mathrm{J}=$ HISIGN & $\mathrm{N}=$ TOSHIBA \\
\hline $\mathrm{C}=$ VIGILANT & $\mathrm{G}=$ MORPHO & $\mathrm{K}=$ COGNITEC & $\mathrm{Q}=$ IMAGUS \\
\hline $\mathrm{D}=$ AYONIX & $\mathrm{H}=3 \mathrm{M}$ COGENT & $\mathrm{L}=$ CYBEREXTRUDER & $\mathrm{R}=$ RANK ONE \\
\hline
\end{tabular}

\begin{tabular}{|l|l|l|l|l|l|}
\hline SET & SCENE & CAMERA & SET & SCENE & CAMERA \\
\hline C & PHOTOJOURNALISM & PRO & T & CONCOURSE & PRO \\
\hline J & PASSENGER LOADING & PRO & H & CONCOURSE & PRO \\
\hline P & SPORTS ARENA & CONSUMER & U & CHOKEPOINT & WEBCAM \\
\hline L & LUGGAGE RACK & WEBCAM & & & \\
\hline
\end{tabular}




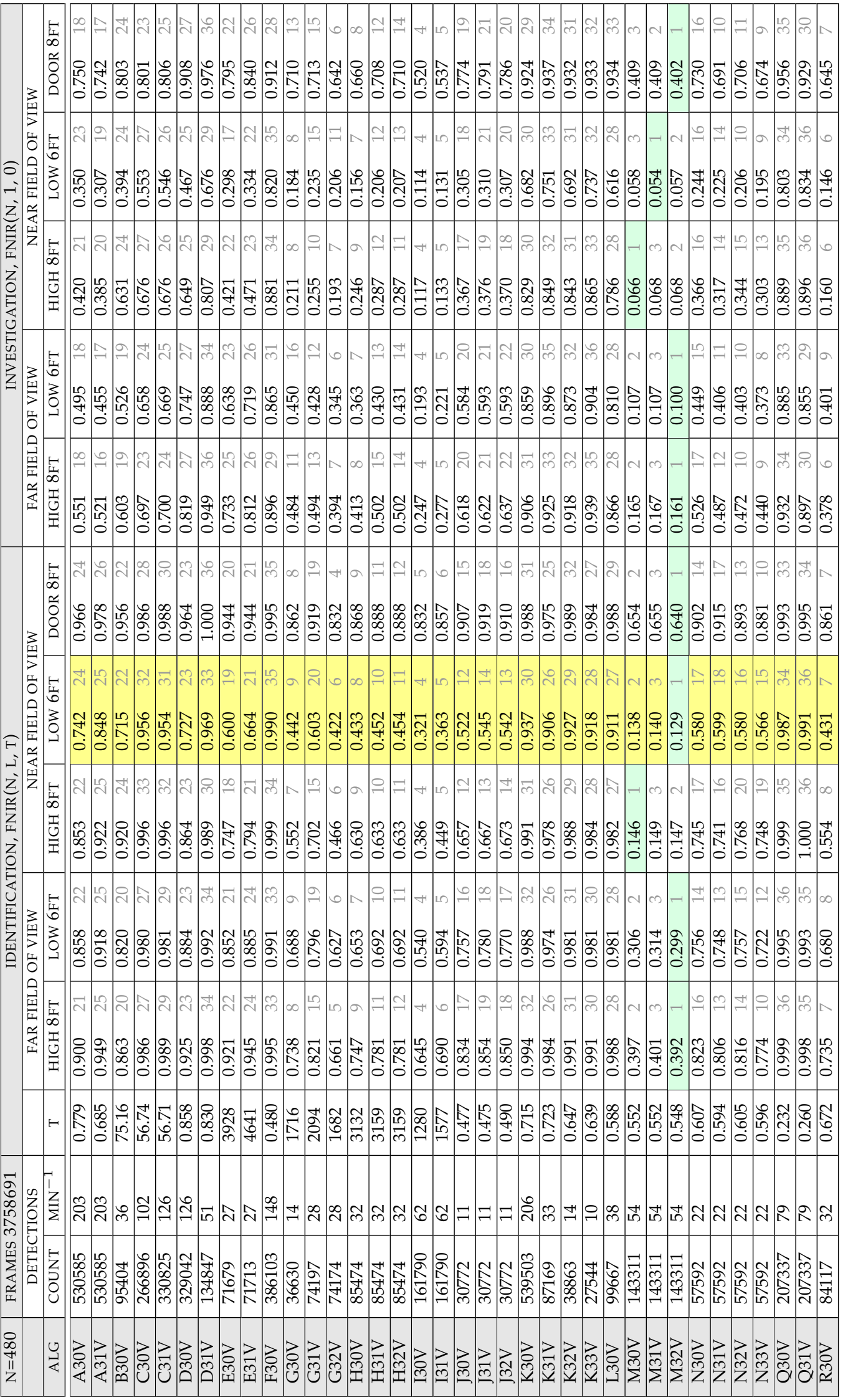

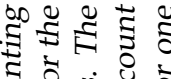

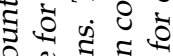
\% ब 8 वृ

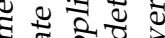
क्षे 든. की $\approx$ का ฐ के . वे चु 8

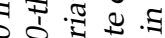
क \& 0 , o

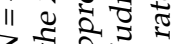
$z \mp \sum_{\pi}^{2}: \frac{\pi}{2}$ $420 \pi .9$ 권휴 ส

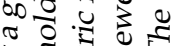
ङ สำ ต

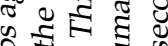
2ฐस

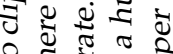
워 $\frac{1}{2} \pi$ บ. เ $\Xi$ : ⿹弋工 䒕结艺

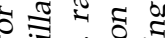
ब 造 के 3

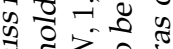
주원원 근 을 放 ปั \& สำ 혀 केषे ฐ ชัণิ ๆ ปี สิ 농ำ

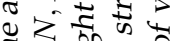
吕采 这文。志

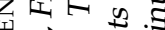
$\cong$ के त 00 \& क छ ڤ ป क \& $\approx \approx 0$

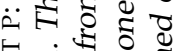

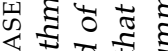
牙 ส 闰

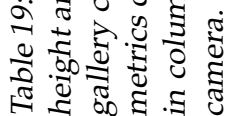

\begin{tabular}{|l|l|l|l|}
\hline \multicolumn{4}{|c|}{ PARTICIPANT KEY } \\
\hline $\mathrm{A}=$ DIGITAL BARRIERS & $\mathrm{E}=$ NEUROTECHNOLOGY & $\mathrm{I}=$ EYEDEA & $\mathrm{M}=$ NEC \\
\hline $\mathrm{B}=$ HBINNO & $\mathrm{F}=$ VAPPLICA & $\mathrm{J}=$ HISIGN & $\mathrm{N}=$ TOSHIBA \\
\hline $\mathrm{C}=$ VIGILANT & $\mathrm{G}=$ MORPHO & $\mathrm{K}=$ COGNITEC & $\mathrm{Q}=$ IMAGUS \\
\hline $\mathrm{D}=$ AYONIX & $\mathrm{H}=$ 3M COGENT & L = CYBEREXTRUDER & $\mathrm{R}=$ RANK ONE \\
\hline
\end{tabular}

\begin{tabular}{|l|l|l|l|l|l|}
\hline SET & SCENE & CAMERA & SET & SCENE & CAMERA \\
\hline C & PHOTOJOURNALISM & PRO & T & CONCOURSE & PRO \\
\hline J & PASSENGER LOADING & PRO & H & CONCOURSE & PRO \\
\hline P & SPORTS ARENA & CONSUMER & U & CHOKEPOINT & WEBCAM \\
\hline L & LUGGAGE RACK & WEBCAM & & & \\
\hline
\end{tabular}




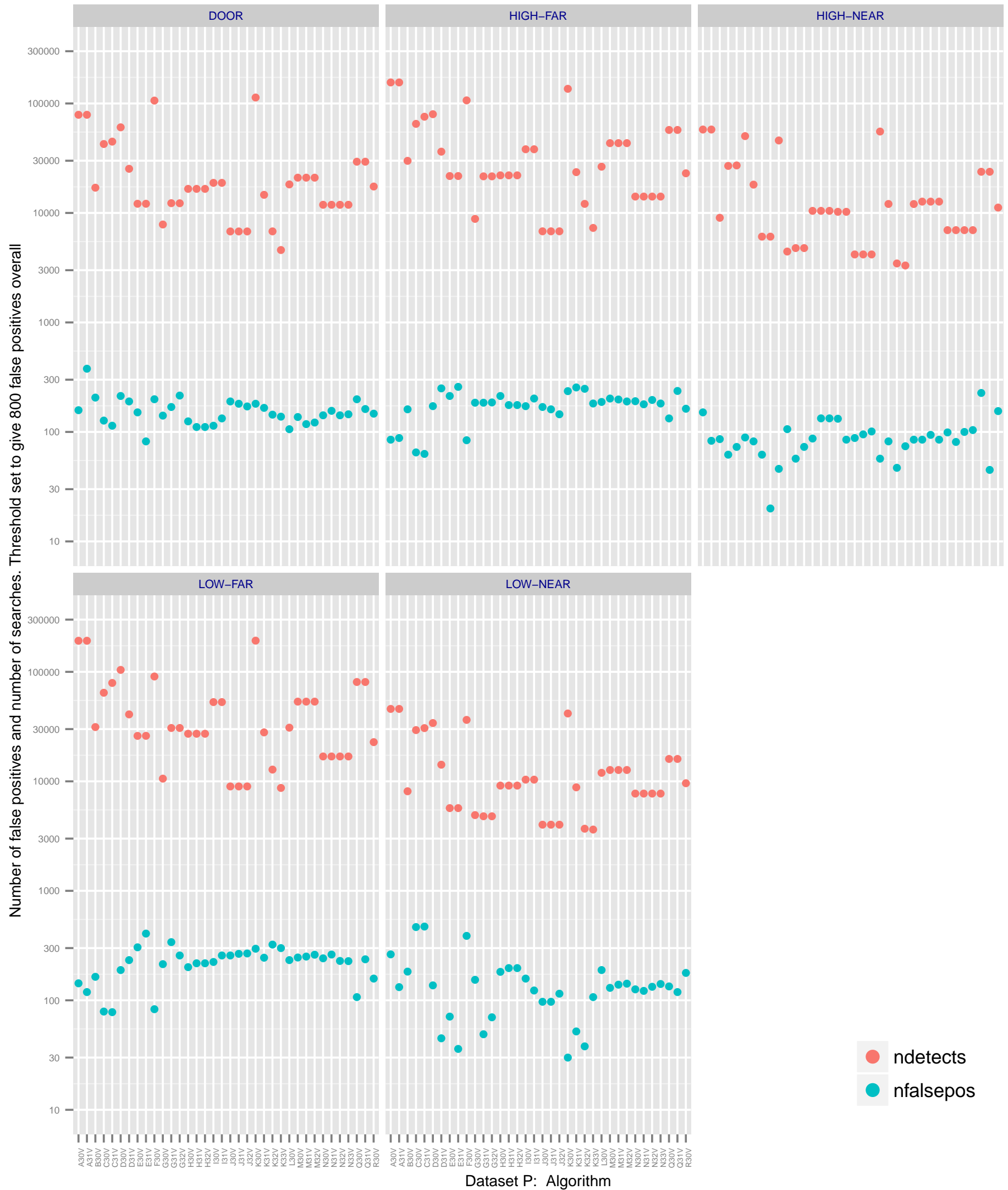

Figure 30: False positives and detection counts for the DATASET P: SPORTS ARENA dataset with threshold set to give 800 false positives over all impostor searches. The panel shows results by camera mount location. Generally more people are detected in the door and "far" field views. The highest false positive counts occur here too, except at the doors, despite the detection counts, possibly due to steeper elevation angles.

\begin{tabular}{|c|c|c|c|c|c|c|c|c|c|}
\hline \multicolumn{4}{|c|}{ PARTICIPANT KEY } & \multirow{2}{*}{\begin{tabular}{|l|} 
SET \\
$\mathrm{C}$ \\
\end{tabular}} & SCENE & \multirow{2}{*}{$\begin{array}{l}\text { CAMERA } \\
\text { PRO }\end{array}$} & \multirow{2}{*}{\begin{tabular}{|l|} 
SET \\
$\mathrm{T}$ \\
\end{tabular}} & \multirow{2}{*}{$\begin{array}{l}\text { SCENE } \\
\text { CONCOURSE }\end{array}$} & \multirow{2}{*}{$\begin{array}{l}\text { CAMERA } \\
\text { PRO }\end{array}$} \\
\hline $\mathrm{A}=$ DIGITAL BARRIERS & $\mathrm{E}=$ NEUROTECHNOLOGY & $\mathrm{I}$ = EYEDEA & $M=N E C$ & & PHOTOJOURNALISM & & & & \\
\hline$C=$ VIGILANT & $\mathrm{G}=\mathrm{MORPHO}$ & $\mathrm{K}=$ COGNITEC & $Q=$ IMAGUS & $\mathrm{P}$ & SPORTS ARENA & CONSUMER & $\mathrm{U}$ & CHOKEPOINT & WEBCAM \\
\hline $\mathrm{D}=\mathrm{AYONIX}$ & $\mathrm{H}=3 \mathrm{M}$ COGENT & $\mathrm{L}=$ CYBEREXTRUDER & $\mathrm{R}=\mathrm{RANK}$ ONE & $\mathrm{L}$ & LUGGAGE RACK & WEBCAM & & & \\
\hline
\end{tabular}



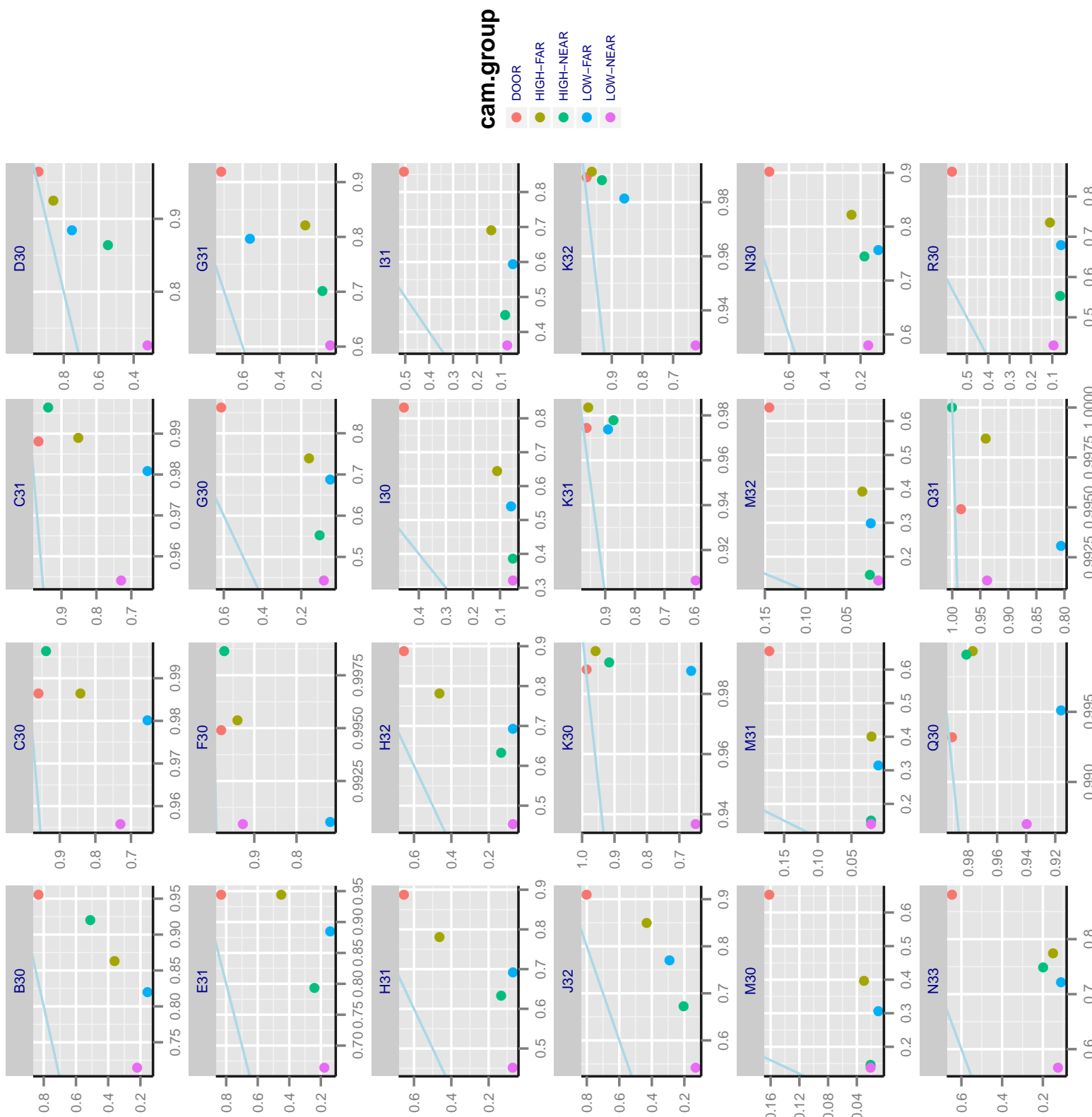

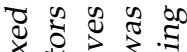

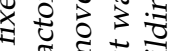

ปร

胥䨔.

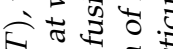

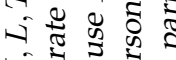

¿

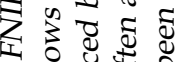

व के के

की

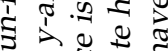

के $\approx$ ส

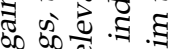

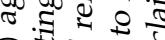

도윰

之สี ฮี ฮี

两 0

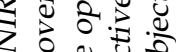

के

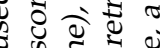

$\infty_{0,0} \approx \pi$

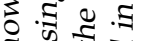

का

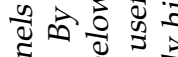

ฉี ชं

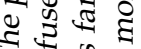

. 苍. 웡

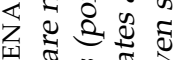

की

至

施公可

के का $\mathbb{E}$

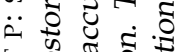

जy

हี Бี

通造

₹ 5 के वे ह

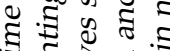
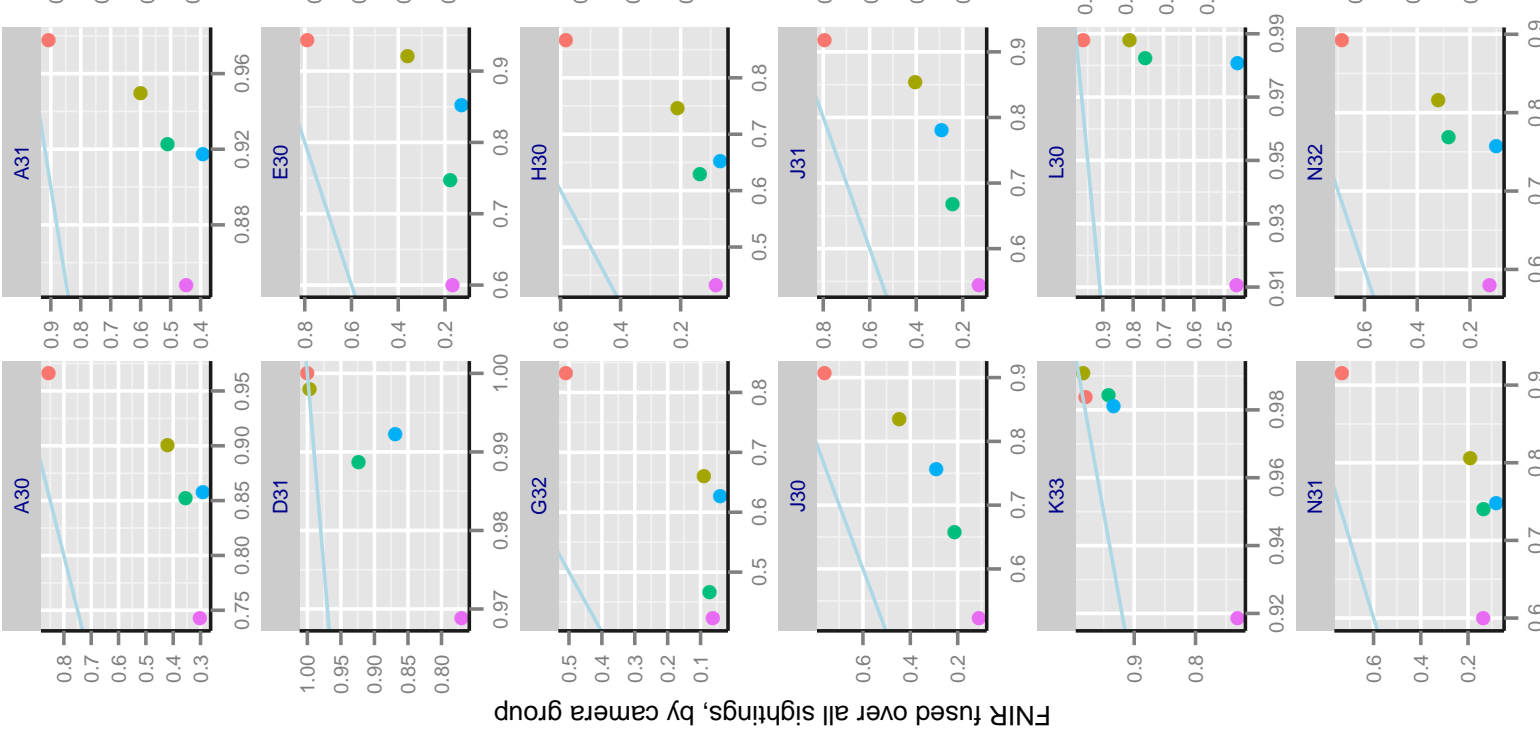

5.50 .5

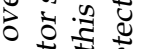

$\approx$ के $\frac{0}{0}=$

क.

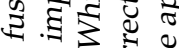

สี

ปั

$+\Delta \overrightarrow{0}$

छ क

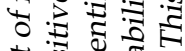

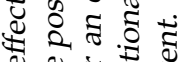

ष

ज沉

ஸें ठ

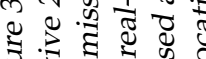

500000.0

PARTICIPANT KEY

\begin{tabular}{|l|l|l|}
\hline $\mathrm{A}=$ DIGITAL BARRIERS & $\mathrm{E}=$ NEUROTECHNOLOGY & $\mathrm{I}=$ EYEDEA
\end{tabular}

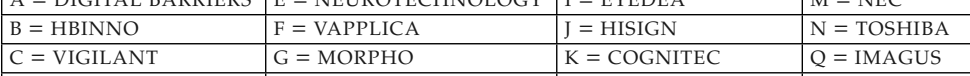

\begin{tabular}{|l|l|l|l|}
\hline $\mathrm{D}=$ AYONIX & $\mathrm{H}=3 \mathrm{M}$ COGENT & $\mathrm{L}=$ CYBEREXTRUDER & $\mathrm{R}=$ RANK ONE \\
\hline
\end{tabular}

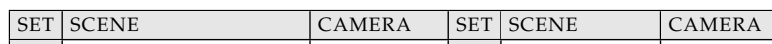
\begin{tabular}{|l|l|l|l|l|l|}
\hline C & PHOTOJOURNALISM & PRO & T & CONCOURSE & PRO \\
\hline J & PASSENGER LOADING & PRO & H & CONCOURSE & PRO \\
\hline P & SPORTS ARENA & CONSUMER & U & CHOKEPOINT & WEBCAM \\
\hline L & LUAC & & & \\
\hline
\end{tabular} 


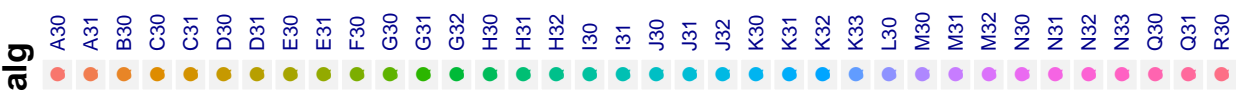

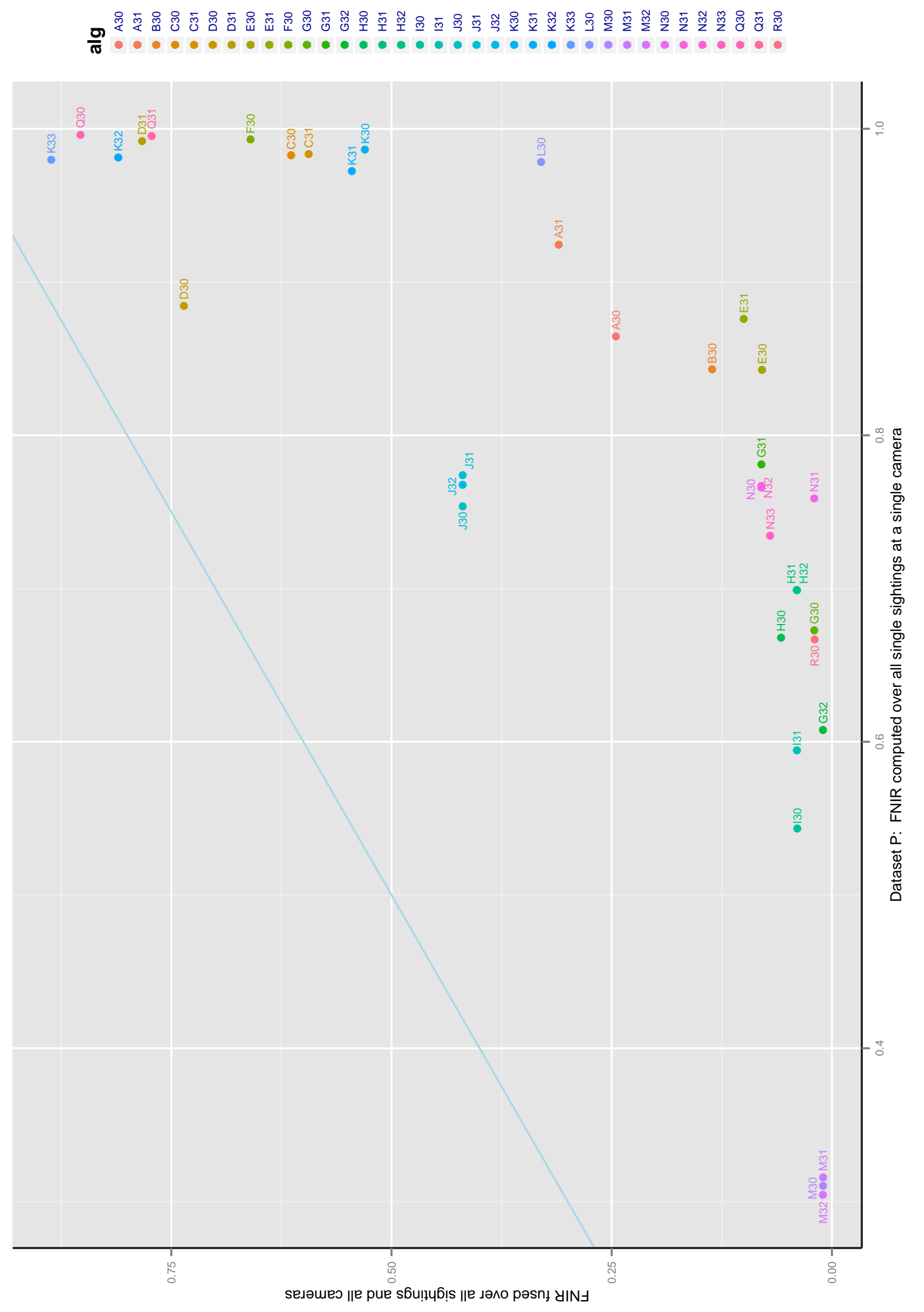

\begin{tabular}{|c|c|c|c|c|c|c|c|c|c|}
\hline \multicolumn{4}{|c|}{ PARTICIPANT KEY } & \multirow{2}{*}{\begin{tabular}{|l|} 
SET \\
$\mathrm{C}$ \\
\end{tabular}} & SCENE & \multirow{2}{*}{\begin{tabular}{|l} 
CAMERA \\
PRO
\end{tabular}} & \multirow{2}{*}{\begin{tabular}{|l|} 
SET \\
$\mathrm{T}$ \\
\end{tabular}} & \multirow{2}{*}{\begin{tabular}{|l|} 
SCENE \\
CONCOURSE \\
\end{tabular}} & \multirow{2}{*}{\begin{tabular}{|l|} 
CAMERA \\
PRO
\end{tabular}} \\
\hline $\mathrm{A}=$ DIGITAL BARRIERS & $\mathrm{E}=\mathrm{NEUROTECHNOLOGY}$ & $\mathrm{I}=$ EYEDEA & $M=N E C$ & & PHOTOJOURNALISM & & & & \\
\hline $\mathrm{C}=$ VIGILANT & $\mathrm{G}=\mathrm{MORPHO}$ & $\mathrm{K}=$ COGNITEC & $\mathrm{Q}=$ IMAGUS & $\mathrm{P}$ & SPORTS ARENA & CONSUMER & $\mathrm{U}$ & CHOKEPOINT & WEBCAM \\
\hline $\mathrm{D}=$ AYONIX & $\mathrm{H}=3 \mathrm{M}$ COGENT & $\mathrm{L}=$ CYBEREXTRUDER & $\mathrm{R}=\mathrm{RANK}$ ONE & $\mathrm{L}$ & LUGGAGE RACK & WEBCAM & & & \\
\hline
\end{tabular}




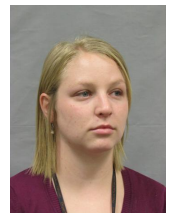

$(-22,0)$

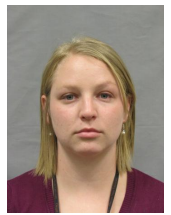

$(0,0)$

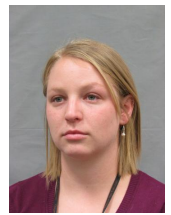

$(22,0)$

Figure 33: DATASET P: SPORTS ARENA : Examples of mugshot-like enrollment images, with nominal pose values (yaw, pitch) provided to the algorithm software. ${ }^{* *}$ The face images here are from a Dataset $P$. Written consent from DHS / S\&T to use these images in public reports was obtained on August 12, 2016. Actor 011414X (Perm Granted).

sees fit. For example, the literature describes methods for synthesizing a single representation from multiple views. This enables comparative performance analysis as follows.

Table 20 shows identification error rates for the "rich" gallery vs. the traditional single still. Figures 34 and 35 show the change in the rank and score of the mate, respectively. The notable observations are:

Value across providers: There are broad accuracy improvements realized by using additional enrollment images. In the investigative mode, rank one miss rates are improved for thirty five algorithms with only one algorithm, $\mathrm{R} 30 \mathrm{~V}$, offering essentially unchanged performance. In the identification mode, $\operatorname{FNIR}(N, L, T)$ is more modestly improved with only N30V giving significantly worse accuracy.

Different value across providers: For some algorithms (providers K, Q, E, D, L) the use of three images per subject gives better mate rankings in many searches. For other algorithms the gains are confined to a smaller number of searches, particularly those from providers $\mathrm{M}, \mathrm{I}, \mathrm{G}, \mathrm{H}, \mathrm{N}$. Rich enrollment gives substantial FNIR(N, 1, 0) error rate reductions: algorithms $\mathrm{H} 31 \mathrm{~V}$ and $\mathrm{H} 32 \mathrm{~V}$ give a fully $41 \%$ fewer errors than with a single full frontal image. For algorithm $\mathrm{A} 31 \mathrm{~V}$ the reduction is $37 \%$, and for $\mathrm{K} 32 \mathrm{~V}, 32 \%$.

The most accurate algorithms, $\mathrm{M} 3 \mathrm{xV}$, already place many mates at rank 1: For $\mathrm{M} 30 \mathrm{~V}, \operatorname{FNIR}(N, 1,0)$ drops from 0.079 to 0.070 a gain of just $11 \%$. This is evident in Figure 34 which shows the mean change in rank of the mate for $\mathrm{M} 30 \mathrm{~V}$ is -0.1 , but is -7.7 for $\mathrm{K} 31 \mathrm{~V}$.

More value in forensics: The accuracy gains are larger in forensics than in watch list surveillance, that is FNIR(N, $R=1, T=0)$ reductions are larger than those for $\operatorname{FNIR}(N, R=L, T)$. Furthermore gains are better still if an investigator is able to review $R=20$ candidates. Here some algorithms (e.g. from developers $K, N, H, A, G, L, Q$ ) give fewer than half as many errors, with $\mathrm{K} 33 \mathrm{~V}$ producing almost one quarter as many errors. This result implies that the effect of three-image enrollment is to improve the rank of the mate without greatly increasing its score - it produces more hits but the hits have modest scores. This is associated also with the two different thresholds for the two galleries. Except for algorithms G30V and G31V, the thresholds for three-image enrollment are higher than those for single-image enrollment, and this harms FNIR to preserve FPIR.

Growth in computational cost: As shown later in Table 35, computational cost increases about linearly in the number of images passed to the template generation function. This cost is incurred once, at enrollment time, and is amortized over searches. See section 6.4.

The benefits are substantial enough that it suggests traditional enrollment processes could be extended to capture additional views of a subject. While the mainline face recognition industry has grown around deduplication, identification

\begin{tabular}{|l|l|l|l|}
\hline \multicolumn{5}{|c|}{ PARTICIPANT KEY } \\
\hline $\mathrm{A}=$ DIGITAL BARRIERS & $\mathrm{E}=$ NEUROTECHNOLOGY & $\mathrm{I}=$ EYEDEA & $\mathrm{M}=$ NEC \\
\hline $\mathrm{B}=$ HBINNO & $\mathrm{F}=$ VAPPLICA & $\mathrm{J}=$ HISIGN & $\mathrm{N}=$ TOSHIBA \\
\hline $\mathrm{C}=$ VIGILANT & $\mathrm{G}=$ MORPHO & $\mathrm{K}=$ COGNITEC & $\mathrm{Q}=$ IMAGUS \\
\hline $\mathrm{D}=$ AYONIX & $\mathrm{H}=$ 3M COGENT & $\mathrm{L}=$ CYBEREXTRUDER & $\mathrm{R}=$ RANK ONE \\
\hline
\end{tabular}

\begin{tabular}{|l|l|l|l|l|l|}
\hline SET & SCENE & CAMERA & SET & SCENE & CAMERA \\
\hline C & PHOTOJOURNALISM & PRO & T & CONCOURSE & PRO \\
\hline $\mathrm{J}$ & PASSENGER LOADING & PRO & H & CONCOURSE & PRO \\
\hline P & SPORTS ARENA & CONSUMER & U & CHOKEPOINT & WEBCAM \\
\hline L & LUGGAGE RACK & WEBCAM & & & \\
\hline
\end{tabular}


and verification of formally standardized ${ }^{14}$ frontal images, the forensics community who, after all, are often left to adjudicate the possibly erroneous results emanating from a face recognition engine, see benefit in having alternative views of subjects' faces. Indeed the result here, that accuracy gains are more substantial in investigative searches (to rank 10 or higher) supports this conclusion. The exact details of what views afford the most benefit is a topic for additional research.

\begin{tabular}{|c|c|c|c|c|c|c|c|c|c|c|}
\hline \multirow{3}{*}{$\mathrm{N}=480$} & \multicolumn{4}{|c|}{ IDENTIFICATION, FNIR(N, L, T) } & \multicolumn{6}{|c|}{ INVESTIGATION, FNIR(N, R, 0) } \\
\hline & \multicolumn{2}{|c|}{ THRESHOLD } & \multicolumn{2}{|c|}{$\operatorname{FNIR}(\mathrm{T}), \operatorname{NFP}(\mathrm{T})=200$} & \multicolumn{2}{|c|}{$\operatorname{FNIR}(\mathrm{R}=1)$} & \multicolumn{2}{|c|}{$\operatorname{FNIR}(\mathrm{R}=10)$} & \multicolumn{2}{|c|}{$\operatorname{FNIR}(\mathrm{R}=20)$} \\
\hline & FF & FF QL QR & FF & FF QL QR & FF & FF QL QR & $\mathrm{FF}$ & FF QL QR & FF & FF QL QR \\
\hline A30V & 0.779 & 0.786 & 0.847 & 0.815 & 0.480 & 0.342 & 0.162 & 0.076 & 0.066 & 0.024 \\
\hline A31V & 0.685 & 0.687 & 0.916 & 0.886 & 0.445 & 0.281 & 0.131 & 0.048 & 0.066 & 0.024 \\
\hline B30V & 75.16 & 76.40 & 0.836 & 0.834 & 0.552 & 0.486 & 0.270 & 0.193 & 0.169 & 0.109 \\
\hline $\mathrm{C} 30 \mathrm{~V}$ & 56.74 & 56.74 & 0.979 & 0.976 & 0.643 & 0.617 & 0.259 & 0.225 & 0.090 & 0.064 \\
\hline C31V & 56.71 & 56.71 & 0.979 & 0.977 & 0.649 & 0.619 & 0.259 & 0.224 & 0.087 & 0.059 \\
\hline D30V & 0.858 & 0.870 & 0.871 & 0.811 & 0.704 & 0.569 & 0.610 & 0.408 & 0.553 & 0.328 \\
\hline D31V & 0.830 & 0.849 & 0.993 & 0.995 & 0.879 & 0.839 & 0.810 & 0.738 & 0.763 & 0.679 \\
\hline E30V & 3928 & 3961 & 0.818 & 0.739 & 0.586 & 0.482 & 0.278 & 0.201 & 0.192 & 0.149 \\
\hline E31V & 4641 & 4655 & 0.852 & 0.771 & 0.653 & 0.524 & 0.318 & 0.194 & 0.219 & 0.124 \\
\hline F30V & 0.480 & 0.481 & 0.992 & 0.992 & 0.849 & 0.835 & 0.500 & 0.445 & 0.267 & 0.205 \\
\hline G30V & 1715 & 1569 & 0.657 & 0.519 & 0.348 & 0.253 & 0.192 & 0.097 & 0.153 & 0.067 \\
\hline G31V & 2093 & 2051 & 0.761 & 0.629 & 0.375 & 0.309 & 0.171 & 0.110 & 0.129 & 0.081 \\
\hline G32V & 1682 & 1812 & 0.588 & 0.609 & 0.291 & 0.231 & 0.137 & 0.093 & 0.104 & 0.064 \\
\hline $\mathrm{H} 30 \mathrm{~V}$ & 3132 & 3151 & 0.601 & 0.527 & 0.302 & 0.226 & 0.116 & 0.085 & 0.080 & 0.060 \\
\hline H31V & 3159 & 3161 & 0.655 & 0.528 & 0.383 & 0.227 & 0.183 & 0.085 & 0.137 & 0.060 \\
\hline $\mathrm{H} 32 \mathrm{~V}$ & 3159 & 3161 & 0.655 & 0.528 & 0.383 & 0.227 & 0.183 & 0.086 & 0.137 & 0.061 \\
\hline $\mathrm{I} 30 \mathrm{~V}$ & 1279 & 1290 & 0.456 & 0.409 & 0.167 & 0.127 & 0.054 & 0.040 & 0.034 & 0.029 \\
\hline I31V & 1577 & 1584 & 0.501 & 0.436 & 0.199 & 0.145 & 0.061 & 0.040 & 0.033 & 0.025 \\
\hline $\mathrm{J} 30 \mathrm{~V}$ & 0.477 & 0.507 & 0.728 & 0.640 & 0.491 & 0.390 & 0.328 & 0.245 & 0.278 & 0.204 \\
\hline J31V & 0.475 & 0.492 & 0.754 & 0.663 & 0.505 & 0.416 & 0.342 & 0.278 & 0.298 & 0.233 \\
\hline $\mathrm{J} 32 \mathrm{~V}$ & 0.490 & 0.518 & 0.738 & 0.649 & 0.494 & 0.391 & 0.327 & 0.256 & 0.276 & 0.210 \\
\hline K30V & 0.715 & 0.720 & 0.975 & 0.933 & 0.792 & 0.536 & 0.564 & 0.245 & 0.416 & 0.163 \\
\hline K31V & 0.723 & 0.729 & 0.950 & 0.873 & 0.834 & 0.584 & 0.678 & 0.279 & 0.593 & 0.170 \\
\hline K32V & 0.647 & 0.657 & 0.965 & 0.902 & 0.812 & 0.555 & 0.643 & 0.280 & 0.540 & 0.191 \\
\hline K33V & 0.639 & 0.649 & 0.962 & 0.898 & 0.833 & 0.604 & 0.691 & 0.319 & 0.610 & 0.221 \\
\hline L30V & 0.588 & 0.798 & 0.972 & 0.963 & 0.785 & 0.590 & 0.407 & 0.177 & 0.257 & 0.041 \\
\hline M30V & 0.552 & 0.554 & 0.235 & 0.202 & 0.079 & 0.070 & 0.036 & 0.031 & 0.026 & 0.022 \\
\hline M31V & 0.552 & 0.555 & 0.242 & 0.210 & 0.080 & 0.069 & 0.033 & 0.030 & 0.025 & 0.022 \\
\hline M32V & 0.548 & 0.550 & 0.236 & 0.204 & 0.081 & 0.070 & 0.033 & 0.027 & 0.024 & 0.020 \\
\hline N30V & 0.607 & 0.621 & 0.734 & 0.833 & 0.382 & 0.364 & 0.211 & 0.113 & 0.159 & 0.065 \\
\hline N31V & 0.594 & 0.594 & 0.727 & 0.678 & 0.342 & 0.271 & 0.175 & 0.124 & 0.136 & 0.088 \\
\hline N32V & 0.605 & 0.608 & 0.725 & 0.650 & 0.351 & 0.225 & 0.187 & 0.097 & 0.147 & 0.066 \\
\hline N33V & 0.596 & 0.596 & 0.706 & 0.653 & 0.325 & 0.259 & 0.174 & 0.121 & 0.140 & 0.088 \\
\hline Q30V & 0.232 & 0.232 & 0.990 & 0.986 & 0.834 & 0.753 & 0.487 & 0.303 & 0.274 & 0.120 \\
\hline Q31V & 0.260 & 0.261 & 0.992 & 0.991 & 0.838 & 0.777 & 0.440 & 0.328 & 0.239 & 0.134 \\
\hline R30V & 0.672 & 0.673 & 0.601 & 0.611 & 0.272 & 0.279 & 0.119 & 0.121 & 0.082 & 0.079 \\
\hline
\end{tabular}

Table 20: For DATASET P: SPORTS ARENA, the accuracy values are FNIR "miss rates" for actors present in a gallery of individuals all of whom enrolled with a single full-frontal (FF) image, or in a separate gallery where individuals are enrolled with three images: one full frontal, and one "quarter left" $(Q L)$ and one "quarter right" $(Q R)$ as in Figure 33. Both galleries hold n $=480$ individuals. The actor video clips are approximately one quarter of the full Dataset $P$ set. The impostor videos are the full set as used in the other Dataset $P$ tables. Cells are shaded red when the richer gallery increases error, and shaded progressively more green when the fractional reduction in FNIR is better than $\{0.8,0.67,0.5\}$. Note the higher thresholds in columns 3 are needed to limit the number of false positives to the same number, 200, over all searches from the non-actor video clips.

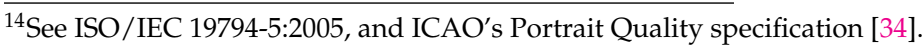

\begin{tabular}{|l|l|l|l|}
\hline \multicolumn{4}{|c|}{ PARTICIPANT KEY } \\
\hline A = DIGITAL BARRIERS & E = NEUROTECHNOLOGY & I EYEDEA & $\mathrm{M}=$ NEC \\
\hline B = HBINNO & F = VAPPLICA & J = HISIGN & = TOSHIBA \\
\hline C = VIGILANT & G = MORPHO & K = COGNITEC & $\mathrm{Q}=$ IMAGUS \\
\hline D = AYONIX & H = 3M COGENT & L = CYBEREXTRUDER & R = RANK ONE \\
\hline
\end{tabular}

\begin{tabular}{|l|l|l|l|l|l|}
\hline SET & SCENE & CAMERA & SET & SCENE & CAMERA \\
\hline C & PHOTOJOURNALISM & PRO & T & CONCOURSE & PRO \\
\hline J & PASSENGER LOADING & PRO & H & CONCOURSE & PRO \\
\hline P & SPORTS ARENA & CONSUMER & U & CHOKEPOINT & WEBCAM \\
\hline L & LUGGAGE RACK & WEBCAM & & & \\
\hline
\end{tabular}



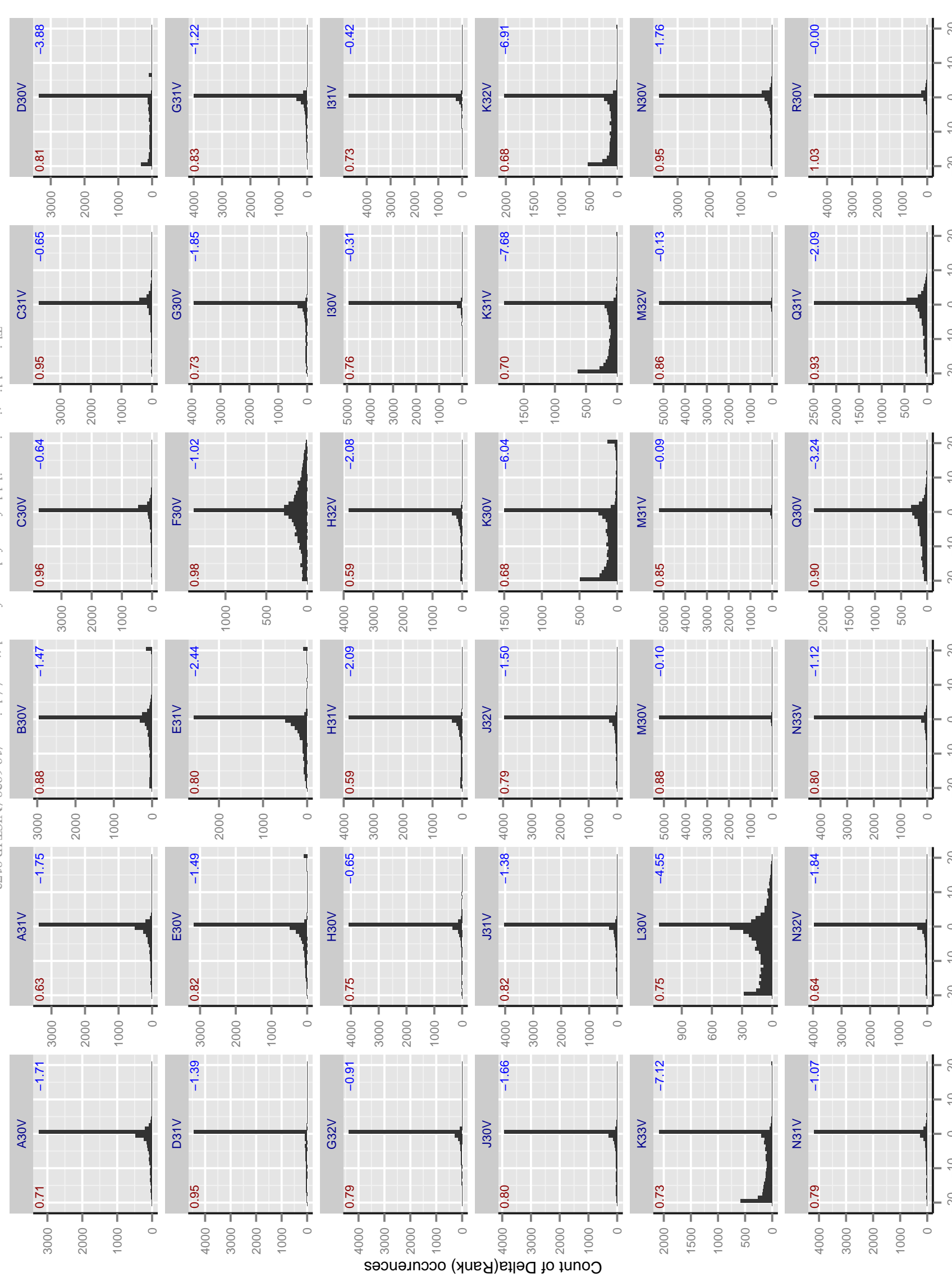

$\mathscr{G} \mathscr{g}$

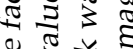

$\approx$ व

की

60.

ว.

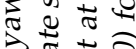

.

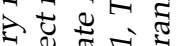

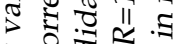

क 8 : $\approx$

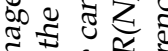

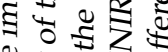

范茫

$\pm \sim$ เ

₹ $\approx$ क

ง

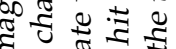

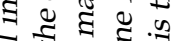

ฮี

ธี

ㄷํำ

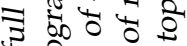

क $: 5.8$

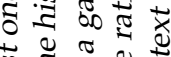

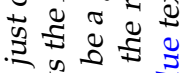

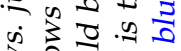

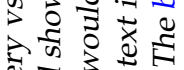

ป

品

ฐ च ฐ

ฐ

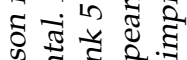

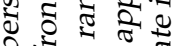

ปั

य 0 .

कृ ส

吉光

จ

ป I \& 11 \&

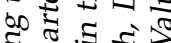

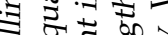

๙ิ

ฮ 둰

Чै।

ปั

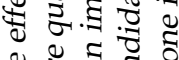

ปี สี สี โี

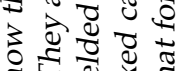

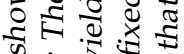

造密方

.

Q

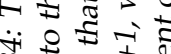

लै 0 ज

๑ :

过 $\frac{\pi}{2} .7 \pm$

PARTICIPANT KEY

\begin{tabular}{|l|l|l|l|}
\hline $\mathrm{A}=$ DIGITAL BARRIERS & $\mathrm{E}=$ NEUROTECHNOLOGY & $\mathrm{I}=$ EYEDEA & $\mathrm{M}=$ NEC \\
\hline $\mathrm{B}=\mathrm{H}$ & $\mathrm{I}$ & $\mathrm{N}$ \\
\hline
\end{tabular}

\begin{tabular}{|l|l|l|l|}
\hline $\mathrm{B}=$ HBINNO & $\mathrm{F}=$ VAPPLICA & $\mathrm{J}=$ HISIGN & $\mathrm{N}=$ TOSHIBA \\
\hline $\mathrm{C}=$ VIGILANT & $\mathrm{G}=$ MORPHO & K = COGNITEC & $\mathrm{Q}=$ IMAGUS \\
\hline $\mathrm{D}=$ AYONIX & $\mathrm{H}=3 \mathrm{M}$ COGENT & CYBEREXTUDER & $\mathrm{R}$ ANK ONE \\
\hline
\end{tabular}

\begin{tabular}{|l|l|l|l}
\hline $\mathrm{D}=$ AYONIX & $\mathrm{H}=3 \mathrm{M}$ COGENT & $\mathrm{L}=$ CYBEREXTRUDER & $\mathrm{R}=$ RANK ON \\
\hline
\end{tabular} 

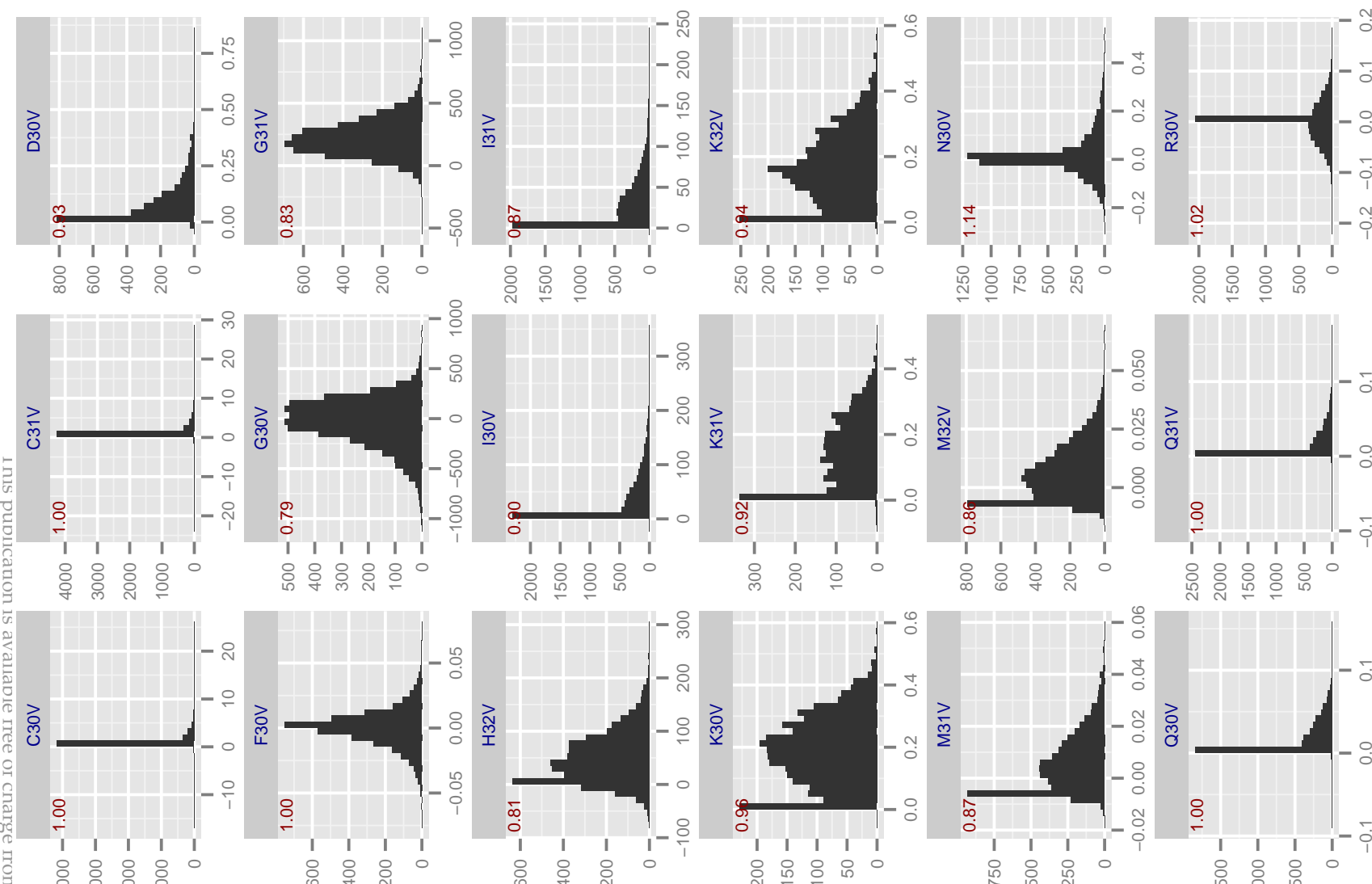

융
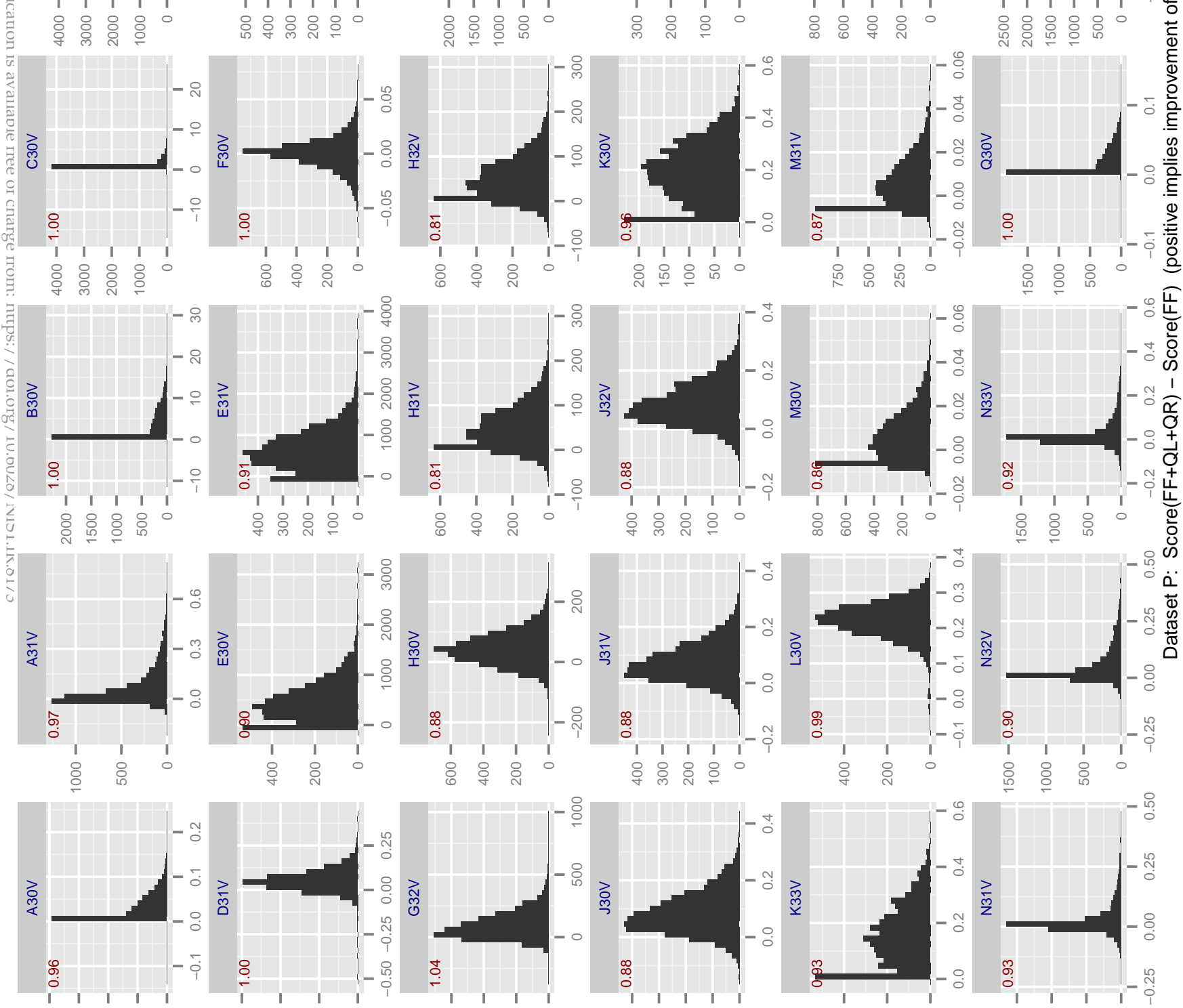

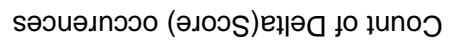

过

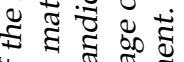

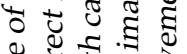

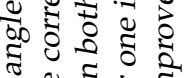

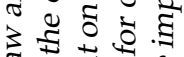

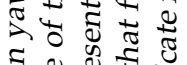

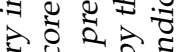
कृ

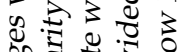

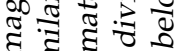
๘ ฐ ฐ ฐ . ฐ ส ₹ ป ปั

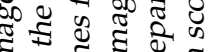
寻 胥 ฐ ธิ口

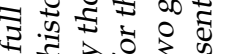

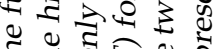

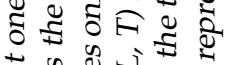

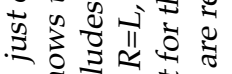
मे क्षे

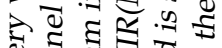
ฮี สี

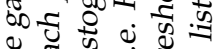
ฐ

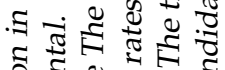

कृ : ญ ปฐ

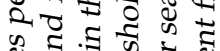
के ส द्व क्षे हू क्षे है \& का वृ द्वे

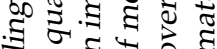
용 ส ป⿻一𠃋十 पे $\frac{2}{5} .5$

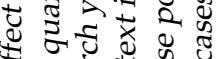

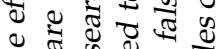
ฐ $\approx$ क 02

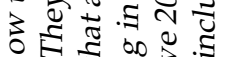

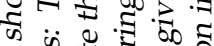
\&

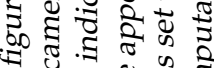
की

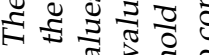
iें

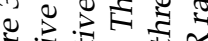

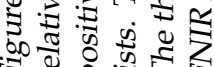




\subsection{DATASET H: TRAVEL WALKWAY}

Overview: This dataset is composed of videos collected using professional grade cameras mounted on the ceiling in a busy travel concourse. The cameras are mounted and configured specifically for the purpose of video surveillance and face recognition. The cameras are placed in three locations, in banks of 4,4 and 2 respectively. Within any one bank, the cameras are mounted next to each other, transverse to their optical axes, which are parallel. Subjects usually walk

towards and underneath the cam-

eras. Occasionally, individuals walk in various transverse directions, including away from the camera. The cameras are visible to the subjects but are almost always ignored. Videos are comprised of clips known to contain actors and clips known not to contain actors. In all cases there are multiple faces visible in the clip. The amount of time a face is fully visible in a scene can vary from approximately 0 to 5

\begin{tabular}{|l|l|l|l|}
\hline Property & Camera bank F & Camera bank R & Camera bank S \\
\hline Cameras & \multicolumn{3}{|c|}{ Avigilon 2.0MP-HD-H264-B1 } \\
\hline Image width & \multicolumn{3}{|c|}{1920} \\
\hline Image height & \multicolumn{3}{|c|}{$20 \mathrm{sec}^{-1}$} \\
\hline Frame rate & \multicolumn{3}{|c|}{ H264 $\mathrm{Mbit} \mathrm{sec}^{-1}$} \\
\hline Nominal bitrate & \multicolumn{3}{|c|}{ Ceiling } \\
\hline Codec & \multicolumn{3}{|c|}{4} \\
\hline \hline Camera mounting & 4 & 2 & 25 \\
\hline Number of cameras & 20 & 14 & 2.10 \\
\hline Max. subjects in view of all cams & 2.65 & 2.3 & {$[2,4]$} \\
\hline Camera height (meters) & {$[2,7]$} & {$[3,8]$} & {$[12,6]$} \\
\hline Range to subject (meters) & {$[28,8]$} & {$[12,4]$} & \\
\hline Camera elevation to frontal face (degrees) & \multicolumn{3}{|c|}{} \\
\hline
\end{tabular}

Table 21: Key imaging properties for DATASET H: TRAVEL WALKWAY results. seconds. Key imaging properties for this dataset are summarized in Table 21.

Enrolled still images: Enrollment images are mugshot-like photos collected under controlled lighting, background, and pose conditions.

Videos: The DATASET H: TRAVEL WALKWAY videos were collected from ten cameras placed on ceilings in a public facility similar to a passenger terminal. The cameras are more expensive and of higher quality than those used for DATASET P: SPORTS ARENA. The videos are also subject to less compression.

Experimental design: Mated scores are computed by searching 439 video clips against three enrolled dataset of portrait face images of subjects known to be in the search videos. The size of the enrollment datasets are $N=\{480,4800,48000\}$. The enrollment database is extended to these sizes by adding high quality frontal portrait photographs from a disjoint background population. Nonmated scores are produced by searching the same videos against the three global nonmated enrollment datasets, of the same $\mathrm{N}$ values.

Key experimental design details are summarized in Table 22.

Results: The results are presented in four tables and one figure.

Tables 23, 2425 give detection counts and recognition accuracy results for enrolled gallery sizes of $\mathrm{N}=480$, 4800 , and 48000, respectively. The tables report both identification mode $\operatorname{FNIR}(N, L, T)$ and investigation mode $\operatorname{FNIR}(N, R, 0)$, for, three thresholds and three ranks of interest, respectively. Note that in high flow, high volume surveillance application, where it will be necessary to minimize false positives, the $\operatorname{FNIR}(N, L, T)$ metric is more relevant than $\operatorname{FNIR}(N, R, 0)$.

Table 26 shows accuracy comparing the three camera banks, corresponding to three different imaging locations and geometries.

\begin{tabular}{|l|l|l|l|}
\hline \multicolumn{5}{|c|}{ PARTICIPANT KEY } \\
\hline $\mathrm{A}=$ DIGITAL BARRIERS & $\mathrm{E}=$ NEUROTECHNOLOGY & $\mathrm{I}=$ EYEDEA & $\mathrm{M}=$ NEC \\
\hline $\mathrm{B}=$ HBINNO & $\mathrm{F}=$ VAPPLICA & $\mathrm{J}=$ HISIGN & $\mathrm{N}=$ TOSHIBA \\
\hline $\mathrm{C}=$ VIGILANT & $\mathrm{G}=$ MORPHO & $\mathrm{K}=$ COGNITEC & $\mathrm{Q}=$ IMAGUS \\
\hline $\mathrm{D}=$ AYONIX & $\mathrm{H}=$ 3M COGENT & $\mathrm{L}=$ CYBEREXTRUDER & $\mathrm{R}=$ RANK ONE \\
\hline
\end{tabular}

\begin{tabular}{|l|l|l|l|l|l|}
\hline SET & SCENE & CAMERA & SET & SCENE & CAMERA \\
\hline C & PHOTOJOURNALISM & PRO & T & CONCOURSE & PRO \\
\hline J & PASSENGER LOADING & PRO & H & CONCOURSE & PRO \\
\hline P & SPORTS ARENA & CONSUMER & U & CHOKEPOINT & WEBCAM \\
\hline L & LUGGAGE RACK & WEBCAM & & & \\
\hline
\end{tabular}




\begin{tabular}{|l|l|}
\hline Quantity & Value or description \\
\hline Mode & Video search to still enrollment \\
\hline Number of actors & 56 \\
\hline Number of non-actors & Many \\
\hline Number of cameras & 10, all ceiling mounted \\
\hline Video duration with actors & 2883 mins \\
\hline Video duration no actors & 8093 mins (but not used) \\
\hline Number of clips actors & 439 \\
\hline Number of clips no actors & 0 \\
\hline Subject motion & Mostly toward and parallel to optical axis. \\
\hline Clip duration (frames) & 4800 fixed \\
\hline Frame rate $\left(s^{-1}\right)$ & 30 \\
\hline Number of enrolled subjects & $480,4800,48000$ \\
\hline Number of enrolled stills & 1 per subject \\
\hline Hroperties of enrolled stills & Frontal, close ICAO compliance; Mean IOD 124 pixels \\
\hline FNIR estimation & Actors present video vs. enrolled gallery \\
\hline FPIR estimation & Actors present video vs. separate non-actor gallery \\
\hline Candidate list length & 20 \\
\hline Number of persons in FOV & {$[0,5]$ typical across FOV } \\
\hline Video ground truth & Style B: See Figure 6 \\
\hline
\end{tabular}

Table 22: Key experimental design for the DATASET H: TRAVEL WALKWAY results.

Figure 36 shows how FNIR and NFP vary at fixed threshold when gallery size changes.

Notable results are as follows.

Detection: Algorithms vary by almost a factor of 20 in the total number of detections they report. Thus, given nearly 20 hours of video, from 10 cameras, the number of detections varies from around 8 per minute to nearly 150 per minute. This variation exists within the $\mathrm{K} 3 \mathrm{xV}$ variants and to a slightly lesser extent across the entire set of algorithms. The reasons for this are to do with:

1. Algorithmic false negatives from failed face detection;

2. Algorithmic false positives from non-faces being reported as faces;

3. Tracking integrity in which algorithms may lose track of an individual over time;

4. Detection policy - minimum spatial resolutions at which to accept a face for processing (see Figure 23)

5. Algorithms may legitimately choose to break a person's track into several parts and generate templates from each - see Figure 3.

While the last two of these are under the control of the algorithm designer, the first three are not readily so. Thus it appears that face detection remains a non-trivial task with a diversity of approaches [13]. We would like to produce a "verbosity" index by normalizing the detection counts by the number of faces actually present. However, per the discussion in section 4.1, that number is unknown and unknowable. Without any assumptions we can only observe the greatly varying numbers of detections. However, in Figure 25, we address this issue using a different dataset for which we do know the actual numbers of people present. There the detection verbosities vary widely too, and increase markedly for subjects who are standing still.

Absolute miss rates: The $\mathrm{M} 3 \mathrm{xV}$ algorithms give the lowest $\operatorname{FNIR}(N, L, T)$ values, for $N=480,4800,48000$. This holds for three operating thresholds corresponding to false positive counts of NFP $=10,100,1000$. There is a very large range in accuracy across the 36 algorithms evaluated - this is typical in independent biometric evaluations.

\begin{tabular}{|l|l|l|l|}
\hline \multicolumn{5}{|c|}{ PARTICIPANT KEY } \\
\hline $\mathrm{A}=$ DIGITAL BARRIERS & $\mathrm{E}=$ NEUROTECHNOLOGY & $\mathrm{I}=$ EYEDEA & $\mathrm{M}=$ NEC \\
\hline $\mathrm{B}=$ HBINNO & $\mathrm{F}=$ VAPPLICA & $\mathrm{J}=$ HISIGN & $\mathrm{N}=$ TOSHIBA \\
\hline $\mathrm{C}=$ VIGILANT & $\mathrm{G}=$ MORPHO & $\mathrm{K}=$ COGNITEC & $\mathrm{Q}=$ IMAGUS \\
\hline $\mathrm{D}=$ AYONIX & $\mathrm{H}=$ 3M COGENT & $\mathrm{L}=$ CYBEREXTRUDER & $\mathrm{R}=$ RANK ONE \\
\hline
\end{tabular}

\begin{tabular}{|l|l|l|l|l|l|}
\hline SET & SCENE & CAMERA & SET & SCENE & CAMERA \\
\hline C & PHOTOJOURNALISM & PRO & T & CONCOURSE & PRO \\
\hline J & PASSENGER LOADING & PRO & H & CONCOURSE & PRO \\
\hline P & SPORTS ARENA & CONSUMER & U & CHOKEPOINT & WEBCAM \\
\hline L & LUGGAGE RACK & WEBCAM & & & \\
\hline
\end{tabular}


The most accurate algorithms give $\operatorname{FNIR}(48000, L, T), \operatorname{NFP}(T)=100$, as low as $0.314(\mathrm{M} 32 \mathrm{~V})$ and 0.599 (G32V) both of which represent useful numbers of identifications in an operational context.

Effect of population size: For the $\mathrm{M} 3 x \mathrm{x}$ algorithms, the error rates for $\operatorname{NFP}(T)=10$ climb very slowly from $\mathrm{N}=$ 480 to 4800 , but more substantially by $N=48000$. This behavior is exhibited by all algorithms, and thus it becomes imperative, even in a high-end purpose-built video surveillance installation equipped with ISO-standard enrolled face images, to maintain $\mathrm{N}$ as small as possible. This should be done by establishing a curation function that limits the number of individuals enrolled, and also removes poor quality imagery.

Comparing rank-based and threshold-based accuracy: The general miss rate metric, $\operatorname{FNIR}(N, R, T)$ is the proportion of actors returned outside the top $\mathrm{R}$ ranks or below threshold $\mathrm{T}$. As with all other datasets, the rank-based investigational accuracy $\operatorname{FNIR}(N, 1,0)$ is better than the threshold-based identification metric $\operatorname{FNIR}(N, L, T)$ essentially because it allows rank-based hits to be "weak", i.e. they are at rank 1, but with a score that is below the high thresholds that are required in surveillance applications. The rank-based metric is useful in "forensic" style searches where there is adequate human labor available to adjudicate candidates produced in manageable low daily volume of searches. Many of the algorithms will produce valuable hits even with $\mathrm{N}=48000$. For example, the 18th most accurate algorithm has FNIR just less than twice that of the most accurate algorithm.

Accuracy relative to Dataset $\mathrm{P}$ : The two datasets $\mathrm{H}$ and $\mathrm{P}$ differ primarily in terms of the expense and properties of the imaging systems, and secondarily in terms of the lighting environment. The interocular distances (see the means tabulated in Appendix I) are higher for $\mathrm{H}$ than $\mathrm{P}$, due to lens configuration and narrower field of view. The accuracy values behave accordingly. Rank 1 accuracy, $\operatorname{FNIR}(480,1,0)$, for dataset $\mathrm{H}$ are two or three times lower than dataset $\mathrm{P}$ except for the $\mathrm{M} 3 \mathrm{xV}$ and $\mathrm{I} 3 \mathrm{xV}$ algorithms which work about as well.

Reasoning about scaling: A primary concern when operating a biometric identification system is about setting the threshold correctly as enrolled population size grows. Typically it is necessary to raise the threshold to maintain a fixed rate of false positives. Practitioners often conceive of false positive outcomes increasing linearly with enrolled population size. From binomial theory, with a fixed threshold $\mathrm{T}, \operatorname{FPIR}(T)=\mathrm{N} \operatorname{FMR}(T)$ where FMR is a fixed oneto-one false match rate. Also FNIR is usually considered to be independent of $\mathrm{N}$, at fixed $\mathrm{T}$, at least if the genuine score computation doesn't depend on the other gallery entries. Figure 36 shows simple binomial theory does not hold. This is not unexpected as some biometric identification systems do not compute the $\mathrm{N}$ scores independently of one another. As such, this consideration of binomial models is therefore naive and moot. However, as is evident in Figure 36 the number of false positives, NFP, does scale linearly with $\mathrm{N}$ for most algorithms, the exceptions being those from developers $\mathrm{G}, \mathrm{H}, \mathrm{M}, \mathrm{N}$. In all cases FNIR varies with $\mathrm{N}$, at fixed threshold. In several cases accuracy with small galleries, $N=480$ is inferior to that with larger $\mathrm{N}$. The conclusion should be that system owners should monitor false positive rates, particularly as $\mathrm{N}$ changes, perhaps by embedding tests into the operational system.

\begin{tabular}{|l|l|l|l|}
\hline \multicolumn{4}{|c|}{ PARTICIPANT KEY } \\
\hline $\mathrm{A}=$ DIGITAL BARRIERS & $\mathrm{E}=$ NEUROTECHNOLOGY & $\mathrm{I}=$ EYEDEA & $\mathrm{M}=$ NEC \\
\hline $\mathrm{B}=$ HBINNO & $\mathrm{F}=$ VAPPLICA & $\mathrm{J}=$ HISIGN & $\mathrm{N}=$ TOSHIBA \\
\hline $\mathrm{C}=$ VIGILANT & $\mathrm{G}=$ MORPHO & $\mathrm{K}=$ COGNITEC & $\mathrm{Q}=$ IMAGUS \\
\hline $\mathrm{D}=$ AYONIX & $\mathrm{H}=3 \mathrm{M}$ COGENT & $\mathrm{L}=$ CYBEREXTRUDER & $\mathrm{R}=$ RANK ONE \\
\hline
\end{tabular}

\begin{tabular}{|l|l|l|l|l|l|}
\hline SET & SCENE & CAMERA & SET & SCENE & CAMERA \\
\hline C & PHOTOJOURNALISM & PRO & T & CONCOURSE & PRO \\
\hline J & PASSENGER LOADING & PRO & H & CONCOURSE & PRO \\
\hline P & SPORTS ARENA & CONSUMER & U & CHOKEPOINT & WEBCAM \\
\hline L & LUGGAGE RACK & WEBCAM & & & \\
\hline
\end{tabular}




\begin{tabular}{|c|c|c|c|c|c|c|c|c|c|c|c|c|c|c|}
\hline \multirow{4}{*}{$\begin{array}{l}\mathrm{N}=480 \\
\text { ALG } \\
\text { A30V }\end{array}$} & \multirow{2}{*}{\multicolumn{2}{|c|}{$\begin{array}{c}\text { NUM ACTORS } 48 \\
\text { DETECTIONS }\end{array}$}} & & \multicolumn{3}{|c|}{ NUM CLIPS 439} & \multicolumn{3}{|c|}{ NUM FRAMES 2107200} & \multicolumn{3}{|c|}{ NUM MINUTES 1170.7} \\
\hline & & & \multirow{2}{*}{\multicolumn{2}{|c|}{ FNIR(T), NFP(T) $=10$}} & \multicolumn{4}{|c|}{ SHOLD BASED IDENTIFICATION } & \multicolumn{6}{|c|}{ RANK BASED INVESTIGATIONS } \\
\hline & NUM & $\mathrm{MIN}^{-1}$ & & & FNIR( & 100 & FNIR( & 000 & FNIR(I & $=0)$ & FNIR(I & $\Gamma=0)$ & FNIR(I & $=0)$ \\
\hline & 81195 & 69.4 & 0.689 & 29 & 0.541 & 28 & 0.396 & 25 & 0.230 & 20 & 0.173 & 14 & 0.133 & 11 \\
\hline A31V & 81195 & 69.4 & 0.650 & 28 & 0.523 & 27 & 0.388 & 24 & 0.218 & 18 & 0.166 & 13 & 0.133 & 10 \\
\hline $\mathrm{B} 30 \mathrm{~V}$ & 16585 & 14.2 & 0.630 & 27 & 0.495 & 24 & 0.375 & 22 & 0.222 & 19 & 0.187 & 16 & 0.142 & 14 \\
\hline C30V & 53549 & 45.7 & 0.827 & 31 & 0.759 & 31 & 0.641 & 32 & 0.324 & 29 & 0.277 & 26 & 0.240 & 24 \\
\hline C31V & 50980 & 43.5 & 0.840 & 32 & 0.782 & 32 & 0.665 & 33 & 0.369 & 30 & 0.323 & 30 & 0.281 & 31 \\
\hline D30V & 136909 & 116.9 & 0.351 & 12 & 0.329 & 16 & 0.317 & 18 & 0.243 & 21 & 0.234 & 22 & 0.185 & 21 \\
\hline D31V & 37923 & 32.4 & 0.609 & 26 & 0.579 & 29 & 0.556 & 30 & 0.310 & 27 & 0.281 & 28 & 0.224 & 23 \\
\hline E30V & 10323 & 8.8 & 0.511 & 23 & 0.391 & 19 & 0.326 & 19 & 0.286 & 23 & 0.265 & 23 & 0.243 & 25 \\
\hline E31V & 10323 & 8.8 & 0.471 & 20 & 0.396 & 20 & 0.336 & 20 & 0.287 & 24 & 0.277 & 27 & 0.249 & 28 \\
\hline F30V & 89883 & 76.8 & 0.988 & 35 & 0.966 & 36 & 0.907 & 36 & 0.671 & 36 & 0.559 & 36 & 0.376 & 36 \\
\hline G30V & 11746 & 10.0 & 0.449 & 19 & 0.339 & 17 & 0.268 & 15 & 0.213 & 17 & 0.201 & 20 & 0.185 & 22 \\
\hline G31V & 11514 & 9.8 & 0.421 & 16 & 0.397 & 21 & 0.353 & 21 & 0.299 & 26 & 0.273 & 25 & 0.246 & 27 \\
\hline G32V & 11075 & 9.5 & 0.342 & 11 & 0.327 & 15 & 0.317 & 17 & 0.313 & 28 & 0.296 & 29 & 0.273 & 30 \\
\hline H30V & 15142 & 12.9 & 1.000 & 36 & 0.964 & 35 & 0.619 & 31 & 0.161 & 8 & 0.142 & 5 & 0.123 & 3 \\
\hline H31V & 15142 & 12.9 & 0.298 & 8 & 0.256 & 7 & 0.201 & 5 & 0.160 & 6 & 0.144 & 6 & 0.123 & 1 \\
\hline H32V & 15142 & 12.9 & 0.298 & 9 & 0.256 & 8 & 0.201 & 6 & 0.160 & 7 & 0.144 & 7 & 0.123 & 2 \\
\hline $\mathrm{I} 30 \mathrm{~V}$ & 14660 & 12.5 & 0.289 & 6 & 0.240 & 4 & 0.197 & 4 & 0.153 & 4 & 0.141 & 3 & 0.132 & 8 \\
\hline I31V & 14660 & 12.5 & 0.310 & 10 & 0.264 & 10 & 0.207 & 7 & 0.154 & 5 & 0.144 & 8 & 0.126 & 6 \\
\hline $\mathrm{J} 30 \mathrm{~V}$ & 12459 & 10.6 & 0.284 & 4 & 0.252 & 5 & 0.230 & 10 & 0.203 & 14 & 0.193 & 19 & 0.163 & 18 \\
\hline $\mathrm{J} 31 \mathrm{~V}$ & 12459 & 10.6 & 0.295 & 7 & 0.255 & 6 & 0.230 & 9 & 0.210 & 16 & 0.191 & 17 & 0.164 & 19 \\
\hline $\mathrm{J} 32 \mathrm{~V}$ & 12459 & 10.6 & 0.287 & 5 & 0.261 & 9 & 0.234 & 14 & 0.209 & 15 & 0.191 & 18 & 0.161 & 17 \\
\hline K30V & 172566 & 147.4 & 0.584 & 25 & 0.521 & 26 & 0.467 & 28 & 0.250 & 22 & 0.225 & 21 & 0.181 & 20 \\
\hline K31V & 10974 & 9.4 & 0.476 & 21 & 0.430 & 22 & 0.378 & 23 & 0.293 & 25 & 0.271 & 24 & 0.246 & 26 \\
\hline K32V & 12178 & 10.4 & 0.550 & 24 & 0.498 & 25 & 0.434 & 27 & 0.385 & 32 & 0.363 & 32 & 0.332 & 33 \\
\hline K33V & 9670 & 8.3 & 0.505 & 22 & 0.456 & 23 & 0.409 & 26 & 0.379 & 31 & 0.364 & 33 & 0.347 & 34 \\
\hline L30V & 21112 & 18.0 & 0.748 & 30 & 0.652 & 30 & 0.544 & 29 & 0.400 & 33 & 0.335 & 31 & 0.259 & 29 \\
\hline M30V & 17334 & 14.8 & 0.206 & 2 & 0.188 & 2 & 0.173 & 3 & 0.145 & 1 & 0.141 & 4 & 0.132 & 9 \\
\hline M31V & 17334 & 14.8 & 0.207 & 3 & 0.191 & 3 & 0.170 & 1 & 0.148 & 3 & 0.138 & 1 & 0.130 & 7 \\
\hline M32V & 17334 & 14.8 & 0.201 & 1 & 0.185 & 1 & 0.172 & 2 & 0.148 & 2 & 0.139 & 2 & 0.124 & 4 \\
\hline N30V & 14594 & 12.5 & 0.410 & 15 & 0.292 & 11 & 0.234 & 13 & 0.178 & 12 & 0.159 & 12 & 0.142 & 15 \\
\hline N31V & 14594 & 12.5 & 0.427 & 17 & 0.316 & 14 & 0.234 & 11 & 0.178 & 11 & 0.156 & 11 & 0.136 & 13 \\
\hline N32V & 14594 & 12.5 & 0.396 & 14 & 0.311 & 13 & 0.234 & 12 & 0.172 & 10 & 0.154 & 10 & 0.135 & 12 \\
\hline N33V & 14594 & 12.5 & 0.387 & 13 & 0.302 & 12 & 0.227 & 8 & 0.169 & 9 & 0.148 & 9 & 0.124 & 5 \\
\hline Q30V & 30436 & 26.0 & 0.972 & 34 & 0.954 & 34 & 0.884 & 35 & 0.633 & 35 & 0.520 & 35 & 0.375 & 35 \\
\hline Q31V & 30436 & 26.0 & 0.941 & 33 & 0.889 & 33 & 0.761 & 34 & 0.530 & 34 & 0.407 & 34 & 0.281 & 32 \\
\hline R30V & 30257 & 25.8 & 0.441 & 18 & 0.356 & 18 & 0.292 & 16 & 0.193 & 13 & 0.173 & 15 & 0.148 & 16 \\
\hline
\end{tabular}

Table 23: For the DATASET H: TRAVEL WALKWAY installation, camera bank all, with 480 subjects enrolled with a frontal still, the values are identification-mode $\operatorname{FNIR}(N, L, T)$ for each algorithm at three different decision thresholdscorresponding to false positive counts of 10,100,1000, and investigation-mode $\operatorname{FNIR}(N, R, 0)$ for ranks 1, 5, 20. Each value is accompanied by an integer ranking across all algorithms. The shading indicates the most important metric to watchlist applications. Ten cameras were used. The detections are summed over all of them. The accuracy values are aggregated over all sightings of all subjects in the field of view of those cameras. Caution: The $R=20$ column is unreliable per the arguments in section 4.4.

\begin{tabular}{|c|c|c|c|c|c|c|c|c|c|}
\hline \multicolumn{4}{|c|}{ PARTICIPANT KEY } & SET & SCENE & CAMERA & SET & SCENE & CAMERA \\
\hline $\mathrm{A}=$ DIGITAL BARRIERS & $\mathrm{E}=$ NEUROTECHNOLOGY & $\mathrm{I}=\mathrm{EYEDEA}$ & $\mathrm{M}=\mathrm{NEC}$ & C & PHOTOJOURNALISM & PRO & $\mathrm{T}$ & CONCOURSE & PRO \\
\hline $\mathrm{B}=\mathrm{HBINNO}$ & $\mathrm{F}=$ VAPPLICA & $\mathrm{J}=\mathrm{HISIGN}$ & $\mathrm{N}=$ TOSHIBA & $\mathrm{J}$ & PASSENGER LOADING & PRO & $\mathrm{H}$ & CONCOURSE & PRO \\
\hline $\mathrm{C}=$ VIGILANT & $\mathrm{G}=\mathrm{MORPHO}$ & $\mathrm{K}=$ COGNITEC & $\mathrm{Q}=\mathrm{IMAGUS}$ & $\mathrm{P}$ & SPORTS ARENA & CONSUMER & $\mathrm{U}$ & CHOKEPOINT & WEBCAM \\
\hline $\mathrm{D}=\mathrm{AYONIX}$ & $\mathrm{H}=3 \mathrm{M}$ COGENT & $\mathrm{L}=$ CYBEREXTRUDER & $\mathrm{R}=\mathrm{RANK}$ ONE & $\mathrm{L}$ & LUGGAGE RACK & WEBCAM & & & \\
\hline
\end{tabular}




\begin{tabular}{|c|c|c|c|c|c|c|c|c|c|c|c|c|c|c|}
\hline \multirow{3}{*}{$\begin{array}{l}\mathrm{N}=4800 \\
\text { ALG }\end{array}$} & \multirow{2}{*}{\multicolumn{2}{|c|}{$\begin{array}{c}\text { NUM ACTORS } 48 \\
\text { DETECTIONS }\end{array}$}} & \multicolumn{3}{|c|}{ NUM FEEDS 41} & \multicolumn{3}{|c|}{ NUM CLIPS 439} & \multicolumn{3}{|c|}{ NUM FRAMES 2107200} & \multicolumn{3}{|c|}{ NUM MINUTES 1170.7} \\
\hline & & & \multicolumn{6}{|c|}{ THRESHOLD BASED IDENTIFICATION } & \multicolumn{6}{|c|}{ RANK BASED INVESTIGATIONS } \\
\hline & NUM & $\mathrm{MIN}^{-1}$ & FNIR( & $=10$ & FNIR( & 100 & FNIR( & 000 & FNIR( & $=0)$ & FNIR(1 & $\mathrm{T}=0)$ & FNIR(1 & $=0)$ \\
\hline $\mathrm{A} 30 \mathrm{~V}$ & 81195 & 69.4 & 0.735 & 27 & 0.639 & 25 & 0.510 & 26 & 0.290 & 21 & 0.252 & 21 & 0.194 & 15 \\
\hline A31V & 81195 & 69.4 & 0.781 & 28 & 0.643 & 26 & 0.496 & 25 & 0.289 & 20 & 0.246 & 19 & 0.194 & 14 \\
\hline B30V & 16585 & 14.2 & 0.656 & 24 & 0.567 & 23 & 0.459 & 22 & 0.298 & 22 & 0.247 & 20 & 0.201 & 16 \\
\hline C30V & 57311 & 49.0 & 0.893 & 32 & 0.840 & 32 & 0.735 & 33 & 0.375 & 28 & 0.336 & 28 & 0.277 & 25 \\
\hline C31V & 54744 & 46.8 & 0.905 & 33 & 0.843 & 33 & 0.735 & 32 & 0.393 & 30 & 0.350 & 29 & 0.290 & 28 \\
\hline D30V & 136909 & 116.9 & 0.496 & 17 & 0.415 & 19 & 0.350 & 19 & 0.267 & 18 & 0.241 & 18 & 0.221 & 21 \\
\hline D31V & 37923 & 32.4 & 0.708 & 25 & 0.658 & 27 & 0.593 & 28 & 0.342 & 26 & 0.302 & 26 & 0.287 & 27 \\
\hline E30V & 10323 & 8.8 & 0.855 & 30 & 0.690 & 29 & 0.422 & 21 & 0.304 & 23 & 0.296 & 24 & 0.277 & 24 \\
\hline E31V & 10323 & 8.8 & 0.889 & 31 & 0.834 & 31 & 0.677 & 31 & 0.319 & 25 & 0.299 & 25 & 0.286 & 26 \\
\hline F30V & 89883 & 76.8 & 0.994 & 35 & 0.987 & 35 & 0.948 & 36 & 0.756 & 35 & 0.655 & 35 & 0.544 & 35 \\
\hline G30V & 11746 & 10.0 & 0.508 & 19 & 0.387 & 17 & 0.290 & 16 & 0.231 & 16 & 0.222 & 17 & 0.207 & 19 \\
\hline G31V & 11514 & 9.8 & 0.498 & 18 & 0.406 & 18 & 0.359 & 20 & 0.307 & 24 & 0.274 & 23 & 0.252 & 23 \\
\hline G32V & 11075 & 9.5 & 0.388 & 12 & 0.353 & 16 & 0.321 & 17 & 0.277 & 19 & 0.261 & 22 & 0.243 & 22 \\
\hline H30V & 15142 & 12.9 & 0.320 & 4 & 0.271 & 4 & 0.225 & 4 & 0.182 & 8 & 0.169 & 8 & 0.159 & 8 \\
\hline H31V & 15142 & 12.9 & 0.323 & 5 & 0.274 & 5 & 0.233 & 6 & 0.182 & 6 & 0.169 & 6 & 0.159 & 6 \\
\hline $\mathrm{H} 32 \mathrm{~V}$ & 15142 & 12.9 & 0.323 & 6 & 0.274 & 6 & 0.233 & 7 & 0.182 & 7 & 0.169 & 7 & 0.159 & 7 \\
\hline I30V & 14660 & 12.5 & 0.382 & 11 & 0.307 & 9 & 0.227 & 5 & 0.166 & 4 & 0.157 & 4 & 0.148 & 4 \\
\hline I31V & 14660 & 12.5 & 0.418 & 13 & 0.314 & 14 & 0.249 & 10 & 0.169 & 5 & 0.157 & 5 & 0.150 & 5 \\
\hline J30V & 12459 & 10.6 & 0.357 & 7 & 0.292 & 7 & 0.252 & 12 & 0.230 & 15 & 0.219 & 16 & 0.203 & 17 \\
\hline J31V & 12459 & 10.6 & 0.363 & 8 & 0.295 & 8 & 0.252 & 11 & 0.225 & 14 & 0.219 & 15 & 0.204 & 18 \\
\hline $\mathrm{J} 32 \mathrm{~V}$ & 12459 & 10.6 & 0.379 & 10 & 0.308 & 11 & 0.261 & 15 & 0.234 & 17 & 0.216 & 14 & 0.213 & 20 \\
\hline K30V & 172566 & 147.4 & 0.717 & 26 & 0.681 & 28 & 0.634 & 29 & 0.381 & 29 & 0.357 & 30 & 0.319 & 30 \\
\hline K31V & 10974 & 9.4 & 0.607 & 22 & 0.556 & 21 & 0.490 & 24 & 0.356 & 27 & 0.329 & 27 & 0.308 & 29 \\
\hline K32V & 12178 & 10.4 & 0.640 & 23 & 0.603 & 24 & 0.533 & 27 & 0.430 & 32 & 0.410 & 32 & 0.385 & 33 \\
\hline K33V & 9670 & 8.3 & 0.601 & 21 & 0.556 & 22 & 0.489 & 23 & 0.419 & 31 & 0.403 & 31 & 0.376 & 31 \\
\hline L30V & 21112 & 18.0 & 0.809 & 29 & 0.736 & 30 & 0.639 & 30 & 0.489 & 33 & 0.440 & 33 & 0.381 & 32 \\
\hline M30V & 17334 & 14.8 & 0.209 & 3 & 0.194 & 2 & 0.182 & 3 & 0.157 & 1 & 0.153 & 3 & 0.144 & 1 \\
\hline M31V & 17334 & 14.8 & 0.209 & 2 & 0.200 & 3 & 0.181 & 2 & 0.160 & 3 & 0.153 & 2 & 0.145 & 3 \\
\hline M32V & 17334 & 14.8 & 0.209 & 1 & 0.191 & 1 & 0.178 & 1 & 0.160 & 2 & 0.153 & 1 & 0.145 & 2 \\
\hline N30V & 14594 & 12.5 & 0.418 & 14 & 0.310 & 12 & 0.252 & 13 & 0.188 & 11 & 0.181 & 11 & 0.164 & 11 \\
\hline N31V & 14594 & 12.5 & 0.455 & 16 & 0.323 & 15 & 0.255 & 14 & 0.196 & 12 & 0.182 & 12 & 0.169 & 12 \\
\hline N32V & 14594 & 12.5 & 0.379 & 9 & 0.307 & 10 & 0.246 & 9 & 0.182 & 9 & 0.181 & 10 & 0.164 & 10 \\
\hline N33V & 14594 & 12.5 & 0.430 & 15 & 0.313 & 13 & 0.239 & 8 & 0.185 & 10 & 0.176 & 9 & 0.160 & 9 \\
\hline Q30V & 30436 & 26.0 & 1.000 & 36 & 1.000 & 36 & 0.947 & 35 & 0.761 & 36 & 0.680 & 36 & 0.578 & 36 \\
\hline Q31V & 30436 & 26.0 & 0.973 & 34 & 0.930 & 34 & 0.849 & 34 & 0.637 & 34 & 0.570 & 34 & 0.461 & 34 \\
\hline R30V & 30068 & 25.7 & 0.563 & 20 & 0.428 & 20 & 0.338 & 18 & 0.222 & 13 & 0.210 & 13 & 0.185 & 13 \\
\hline
\end{tabular}

Table 24: For the DATASET H: TRAVEL WALKWAY installation, camera bank all, with 4800 subjects enrolled with a frontal still, the values are identification-mode $\operatorname{FNIR}(N, L, T)$ for each algorithm at three different decision thresholdscorresponding to false positive counts of 10,100,1000, and investigation-mode $\operatorname{FNIR}(N, R, 0)$ for ranks 1, 5, 20. Each value is accompanied by an integer ranking across all algorithms. The shading indicates the most important metric to watchlist applications. Ten cameras were used. The detections are summed over all of them. The accuracy values are aggregated over all sightings of all subjects in the field of view of those cameras.

\begin{tabular}{|c|c|c|c|c|c|c|c|c|c|}
\hline \multicolumn{4}{|c|}{ PARTICIPANT KEY } & SET & SCENE & CAMERA & SET & SCENE & CAMERA \\
\hline $\mathrm{A}=$ DIGITAL BARRIERS & $\mathrm{E}=$ NEUROTECHNOLOGY & $\mathrm{I}=\mathrm{EYEDEA}$ & $M=N E C$ & C & PHOTOJOURNALISM & PRO & $\mathrm{T}$ & CONCOURSE & PRO \\
\hline $\mathrm{B}=\mathrm{HBINNO}$ & $\mathrm{F}=$ VAPPLICA & $\mathrm{J}=\mathrm{HISIGN}$ & $\mathrm{N}=$ TOSHIBA & $\mathrm{J}$ & PASSENGER LOADING & PRO & $\mathrm{H}$ & CONCOURSE & PRO \\
\hline$C=$ VIGILANT & $\mathrm{G}=\mathrm{MORPHO}$ & $\mathrm{K}=$ COGNITEC & $\mathrm{Q}=$ IMAGUS & $P$ & SPORTS ARENA & CONSUMER & $\mathrm{U}$ & CHOKEPOINT & WEBCAM \\
\hline $\mathrm{D}=\mathrm{AYONIX}$ & $\mathrm{H}=3 \mathrm{M}$ COGENT & $\mathrm{L}=$ CYBEREXTRUDER & $\mathrm{R}=\mathrm{RANK}$ ONE & $\mathrm{L}$ & LUGGAGE RACK & WEBCAM & & & \\
\hline
\end{tabular}




\begin{tabular}{|c|c|c|c|c|c|c|c|c|c|c|c|c|c|c|}
\hline \multirow{3}{*}{$\begin{array}{l}\mathrm{N}=48000 \\
\text { ALG }\end{array}$} & \multirow{2}{*}{\multicolumn{2}{|c|}{$\begin{array}{c}\text { NUM ACTORS } 48 \\
\text { DETECTIONS }\end{array}$}} & \multicolumn{3}{|c|}{ NUM FEEDS 41} & \multicolumn{3}{|c|}{ NUM CLIPS 439} & \multicolumn{3}{|c|}{ NUM FRAMES 2107200} & \multicolumn{3}{|c|}{ NUM MINUTES 1170.7} \\
\hline & & & \multicolumn{6}{|c|}{ THRESHOLD BASED IDENTIFICATION } & \multicolumn{6}{|c|}{ RANK BASED INVESTIGATIONS } \\
\hline & NUM & $\mathrm{MIN}^{-1}$ & FNIR( & $=10$ & FNIR( & 100 & FNIR( & 000 & FNIR( & $=0)$ & FNIR( & $\Gamma=0)$ & FNIR( & $=0)$ \\
\hline A30V & 81195 & 69.4 & 0.960 & 27 & 0.921 & 27 & 0.868 & 28 & 0.744 & 30 & 0.674 & 30 & 0.610 & 30 \\
\hline A31V & 81195 & 69.4 & 1.000 & 34 & 0.994 & 33 & 0.967 & 33 & 0.733 & 29 & 0.667 & 29 & 0.610 & 29 \\
\hline B30V & 16585 & 14.2 & 0.930 & 26 & 0.907 & 26 & 0.843 & 26 & 0.701 & 28 & 0.628 & 28 & 0.554 & 27 \\
\hline C30V & 59390 & 50.7 & 0.996 & 30 & 0.987 & 32 & 0.960 & 31 & 0.821 & 32 & 0.796 & 32 & 0.761 & 32 \\
\hline C31V & 56856 & 48.6 & 0.999 & 31 & 0.987 & 31 & 0.961 & 32 & 0.841 & 33 & 0.815 & 33 & 0.791 & 33 \\
\hline D30V & 136909 & 116.9 & 0.828 & 17 & 0.724 & 16 & 0.587 & 12 & 0.416 & 10 & 0.379 & 10 & 0.361 & 11 \\
\hline D31V & 37923 & 32.4 & 0.911 & 25 & 0.819 & 22 & 0.741 & 23 & 0.450 & 11 & 0.412 & 12 & 0.394 & 15 \\
\hline E30V & 10323 & 8.8 & 0.999 & 32 & 0.973 & 29 & 0.612 & 13 & 0.412 & 9 & 0.370 & 9 & 0.327 & 9 \\
\hline E31V & 10323 & 8.8 & 0.990 & 29 & 0.973 & 30 & 0.871 & 29 & 0.526 & 20 & 0.443 & 15 & 0.354 & 10 \\
\hline F30V & 89883 & 76.8 & 1.000 & 36 & 0.999 & 35 & 0.996 & 35 & 0.956 & 35 & 0.936 & 36 & 0.917 & 36 \\
\hline G30V & 11746 & 10.0 & 0.791 & 11 & 0.716 & 13 & 0.625 & 16 & 0.481 & 16 & 0.452 & 17 & 0.427 & 19 \\
\hline G31V & 11514 & 9.8 & 0.710 & 5 & 0.613 & 8 & 0.545 & 9 & 0.470 & 14 & 0.436 & 14 & 0.385 & 14 \\
\hline G32V & 11075 & 9.5 & 0.670 & 4 & 0.599 & 4 & 0.532 & 8 & 0.476 & 15 & 0.450 & 16 & 0.409 & 17 \\
\hline H30V & 15142 & 12.9 & 0.763 & 10 & 0.604 & 5 & 0.434 & 4 & 0.289 & 6 & 0.267 & 5 & 0.244 & 4 \\
\hline H31V & 15142 & 12.9 & 0.763 & 8 & 0.612 & 6 & 0.443 & 5 & 0.289 & 4 & 0.267 & 4 & 0.244 & 2 \\
\hline $\mathrm{H} 32 \mathrm{~V}$ & 15142 & 12.9 & 0.763 & 9 & 0.612 & 7 & 0.443 & 6 & 0.289 & 5 & 0.268 & $\frac{1}{6}$ & 0.244 & 3 \\
\hline $\mathrm{I} 30 \mathrm{~V}$ & 14660 & 12.5 & 0.739 & 6 & 0.656 & 10 & 0.572 & 10 & 0.502 & 18 & 0.492 & 21 & 0.480 & 24 \\
\hline $\mathrm{I} 31 \mathrm{~V}$ & 14660 & 12.5 & 0.884 & 22 & 0.821 & 23 & 0.701 & 21 & 0.545 & 23 & 0.501 & 23 & 0.481 & 25 \\
\hline $\mathrm{J} 30 \mathrm{~V}$ & 12459 & 10.6 & 0.828 & 18 & 0.757 & 18 & 0.659 & 19 & 0.532 & 21 & 0.495 & 22 & 0.447 & 22 \\
\hline $\mathrm{J} 31 \mathrm{~V}$ & 12459 & 10.6 & 0.827 & 16 & 0.753 & 17 & 0.646 & 18 & 0.538 & 22 & 0.489 & 20 & 0.434 & 20 \\
\hline $\mathrm{J} 32 \mathrm{~V}$ & 12459 & 10.6 & 0.796 & 13 & 0.719 & 15 & 0.628 & 17 & 0.489 & 17 & 0.459 & 18 & 0.406 & 16 \\
\hline K30V & 172566 & 147.4 & 0.898 & 24 & 0.879 & 25 & 0.853 & 27 & 0.601 & 26 & 0.563 & 26 & 0.511 & 26 \\
\hline K31V & 10974 & 9.4 & 0.846 & 20 & 0.813 & 21 & 0.756 & 24 & 0.597 & 25 & 0.542 & 25 & 0.447 & 21 \\
\hline K32V & 12178 & 10.4 & 0.887 & 23 & 0.846 & 24 & 0.776 & 25 & 0.636 & 27 & 0.606 & 27 & 0.569 & 28 \\
\hline K33V & 9670 & 8.3 & 0.841 & 19 & 0.787 & 19 & 0.701 & 20 & 0.564 & 24 & 0.517 & 24 & 0.470 & 23 \\
\hline L30V & 21112 & 18.0 & 0.966 & 28 & 0.945 & 28 & 0.899 & 30 & 0.813 & 31 & 0.767 & 31 & 0.704 & 31 \\
\hline M30V & 17334 & 14.8 & 0.350 & 2 & 0.317 & 3 & 0.292 & 3 & 0.259 & 3 & 0.252 & 2 & 0.246 & 6 \\
\hline M31V & 17334 & 14.8 & 0.347 & 1 & 0.314 & 2 & 0.292 & 2 & 0.258 & 2 & 0.255 & 3 & 0.246 & 5 \\
\hline M32V & 17334 & 14.8 & 0.353 & 3 & 0.314 & 1 & 0.280 & 1 & 0.258 & 1 & 0.249 & 1 & 0.243 & 1 \\
\hline N30V & 14594 & 12.5 & 0.757 & 7 & 0.641 & 9 & 0.514 & 7 & 0.366 & 7 & 0.336 & 7 & 0.298 & 7 \\
\hline N31V & 14594 & 12.5 & 0.803 & 15 & 0.719 & 14 & 0.612 & 14 & 0.452 & 13 & 0.413 & 13 & 0.372 & 13 \\
\hline N32V & 14594 & 12.5 & 0.794 & 12 & 0.689 & 11 & 0.575 & 11 & 0.397 & 8 & 0.344 & 8 & 0.299 & 8 \\
\hline N33V & 14594 & 12.5 & 0.801 & 14 & 0.713 & 12 & 0.616 & 15 & 0.452 & 12 & 0.410 & 11 & 0.366 & 12 \\
\hline Q30V & 30436 & 26.0 & 1.000 & 33 & 1.000 & 36 & 1.000 & 36 & 0.957 & 36 & 0.930 & 34 & 0.898 & 34 \\
\hline Q31V & 30436 & 26.0 & 1.000 & 35 & 0.997 & 34 & 0.990 & 34 & 0.941 & 34 & 0.930 & 35 & 0.905 & 35 \\
\hline R30V & 29040 & 24.8 & 0.881 & 21 & 0.810 & 20 & 0.708 & 22 & 0.507 & 19 & 0.461 & 19 & 0.409 & 18 \\
\hline
\end{tabular}

Table 25: For the DATASET H: TRAVEL WALKWAY installation, camera bank all, with 48000 subjects enrolled with a frontal still, the values are identification-mode $\operatorname{FNIR}(N, L, T)$ for each algorithm at three different decision thresholdscorresponding to false positive counts of 10,100,1000, and investigation-mode $\operatorname{FNIR}(N, R, 0)$ for ranks 1, 5, 20. Each value is accompanied by an integer ranking across all algorithms. The shading indicates the most important metric to watchlist applications. Ten cameras were used. The detections are summed over all of them. The accuracy values are aggregated over all sightings of all subjects in the field of view of those cameras.

\begin{tabular}{|c|c|c|c|c|c|c|c|c|c|}
\hline \multicolumn{4}{|c|}{ PARTICIPANT KEY } & \multirow{2}{*}{\begin{tabular}{|l|} 
SET \\
$\mathrm{C}$ \\
\end{tabular}} & SCENE & \multirow{2}{*}{\begin{tabular}{|l|} 
CAMERA \\
PRO
\end{tabular}} & \multirow{2}{*}{\begin{tabular}{|l} 
SET \\
$\mathrm{T}$
\end{tabular}} & \multirow{2}{*}{$\begin{array}{l}\text { SCENE } \\
\text { CONCOURSE }\end{array}$} & \multirow{2}{*}{\begin{tabular}{|l|} 
CAMERA \\
PRO
\end{tabular}} \\
\hline $\mathrm{A}=$ DIGITAL BARRIERS & $E=$ NEUROTECHNOLOGY & $I=$ EYEDEA & $M=N E C$ & & PHOTOJOURNALISM & & & & \\
\hline$C=$ VIGILANT & $G=$ MORPHO & $\mathrm{K}=$ COGNITEC & $\mathrm{Q}=$ IMAGUS & $\mathrm{P}$ & SPORTS ARENA & CONSUMER & $\mathrm{U}$ & CHOKEPOINT & WEBCAM \\
\hline $\mathrm{D}=$ AYONIX & $\mathrm{H}=3 \mathrm{M}$ COGENT & $\mathrm{L}=$ CYBEREXTRUDER & $\mathrm{R}=\mathrm{RANK}$ ONE & $\mathrm{L}$ & LUGGAGE RACK & WEBCAM & & & \\
\hline
\end{tabular}




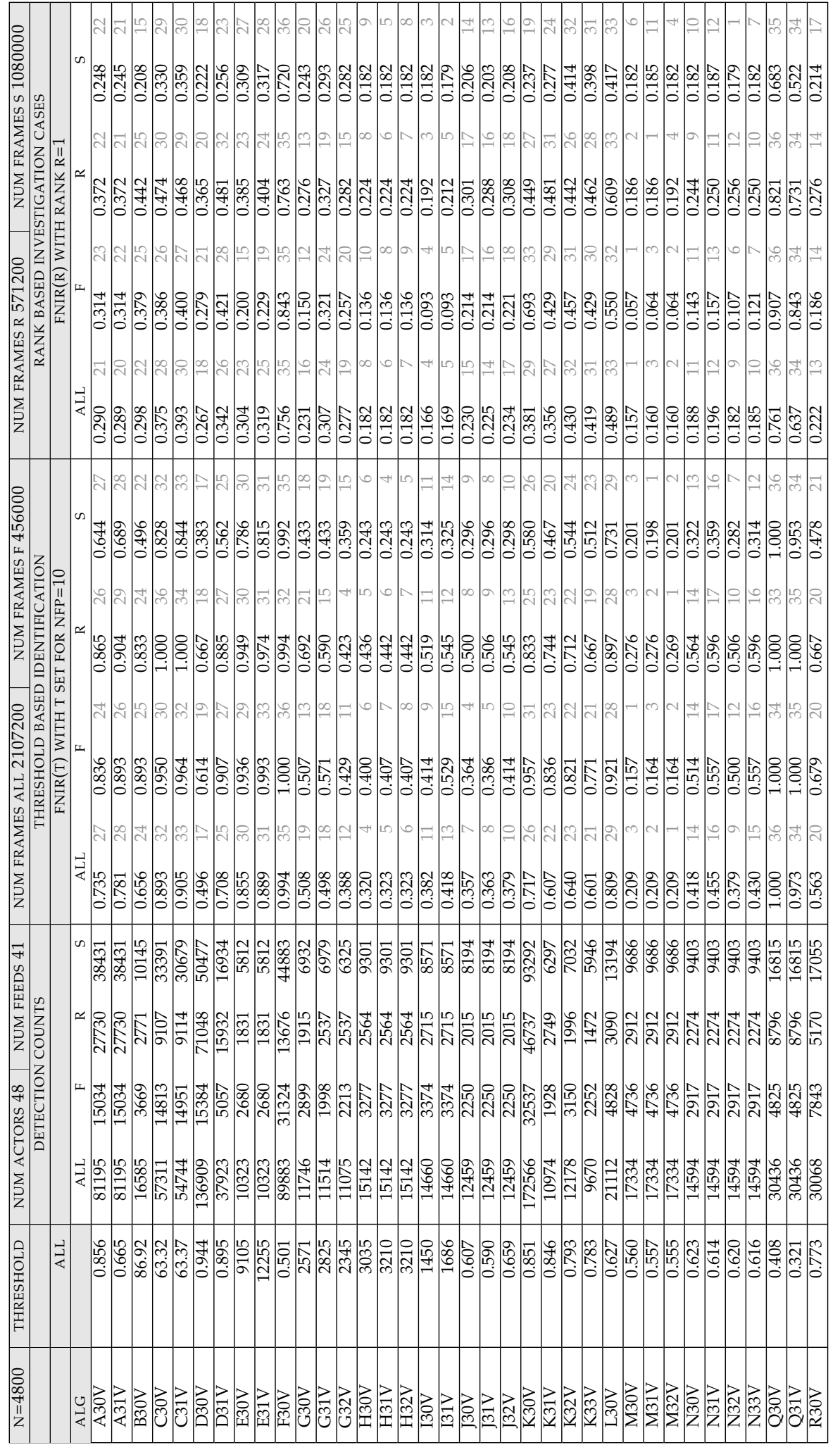

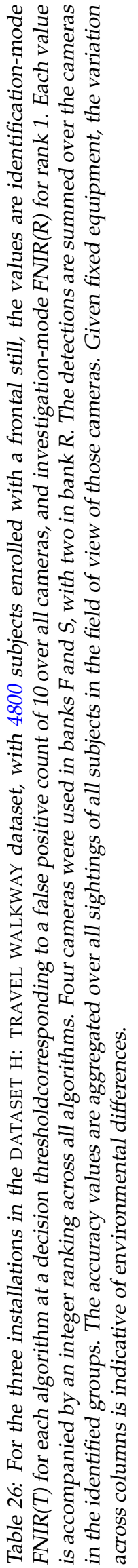

\begin{tabular}{|l|l|l|l|}
\hline \multicolumn{4}{|c|}{ PARTICIPANT KEY } \\
\hline $\mathrm{A}=$ DIGITAL BARRIERS & $\mathrm{E}=$ NEUROTECHNOLOGY & $\mathrm{I}=$ EYEDEA & $\mathrm{M}=$ NEC \\
\hline $\mathrm{B}=$ HBINNO & $\mathrm{F}=$ VAPPLICA & $\mathrm{J}=$ HISIGN & $\mathrm{N}=$ TOSHIBA \\
\hline $\mathrm{C}=$ VIGILANT & $\mathrm{G}=$ MORPHO & $\mathrm{K}=$ COGNITEC & $\mathrm{Q}=$ IMAGUS \\
\hline $\mathrm{D}=$ AYONIX & $\mathrm{H}=$ 3M COGENT & L = CYBEREXTRUDER & $\mathrm{R}=$ RANK ONE \\
\hline
\end{tabular}

\begin{tabular}{|l|l|l|l|l|l|}
\hline SET & SCENE & CAMERA & SET & SCENE & CAMERA \\
\hline C & PHOTOJOURNALISM & PRO & T & CONCOURSE & PRO \\
\hline J & PASSENGER LOADING & PRO & H & CONCOURSE & PRO \\
\hline P & SPORTS ARENA & CONSUMER & U & CHOKEPOINT & WEBCAM \\
\hline L & LUGGAGE RACK & WEBCAM & & & \\
\hline
\end{tabular}


$480 \triangle 4800 \square 48000$

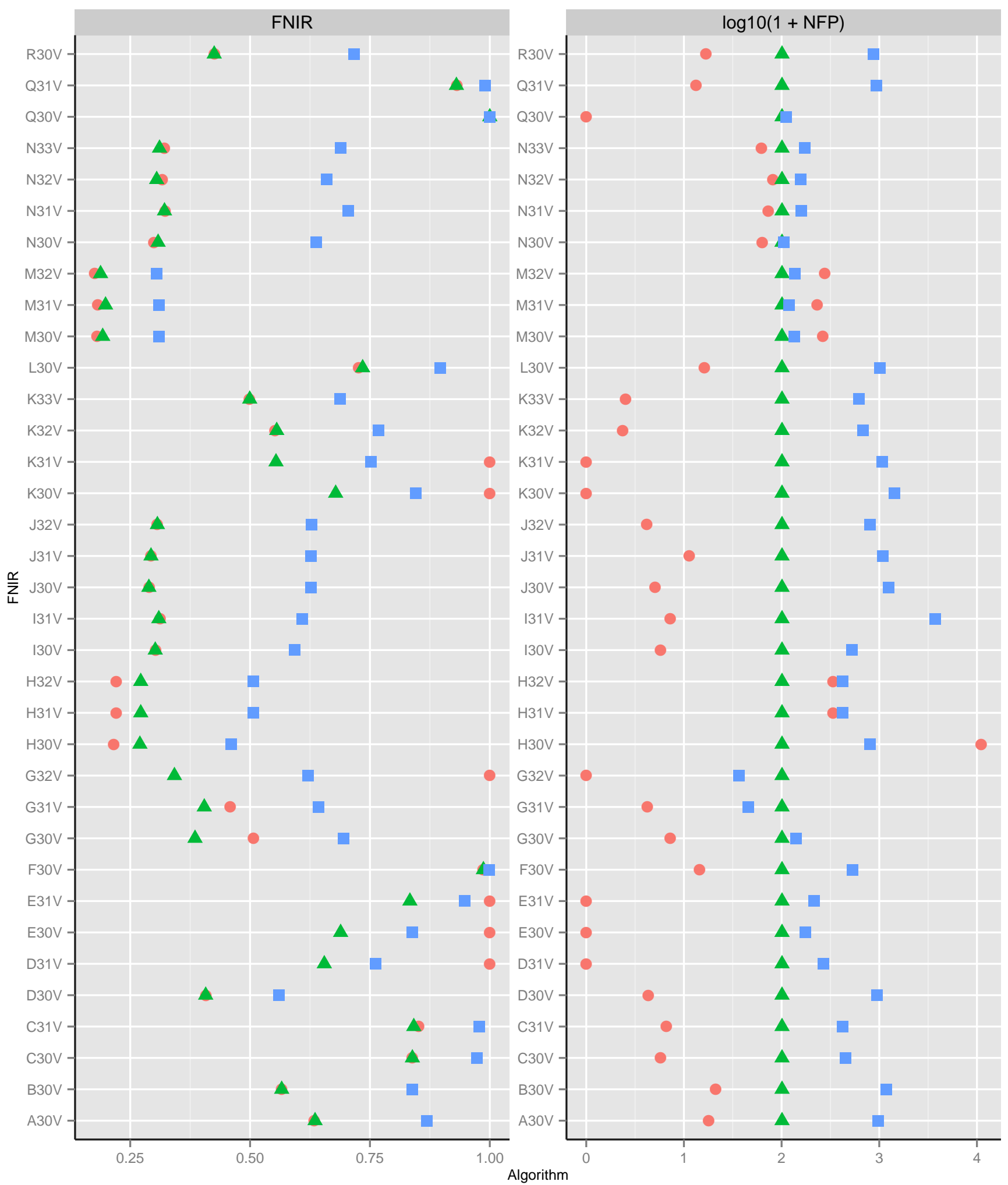

Figure 36: Over all cameras in the DATASET H: TRAVEL WALKWAY collection, the dots show accuracy for $N=\{48000,4800,480\}$, at a single global decision threshold set to produce $\operatorname{NFP}(T)=100$ false positives over all searches of video templates against impostor galleries of size $N=4800$. The left panel shows FNIR $(N, L, T)$. The right panel shows $\log _{10}(1+N F P(T))$. Simple binomial theory would dictate linear growth NFP with $N$, and FNIR independent of $N$.

\begin{tabular}{|l|l|l|l|}
\hline \multicolumn{4}{|c|}{ PARTICIPANT KEY } \\
\hline A = DIGITAL BARRIERS & E = NEUROTECHNOLOGY & I = EYEDEA & $\mathrm{M}=$ NEC \\
\hline B = HBINNO & F = VAPPLICA & I = HISIGN & $\mathrm{N}=$ TOSHIBA \\
\hline C = VIGILANT & G = MORPHO & K = COGNITEC & $\mathrm{Q}=$ IMAGUS \\
\hline D = AYONIX & $\mathrm{H}=$ 3M COGENT & $\mathrm{L}=$ CYBEREXTRUDER & $\mathrm{R}=$ RANK ONE \\
\hline
\end{tabular}

\begin{tabular}{|l|l|l|l|l|l|}
\hline SET & SCENE & CAMERA & SET & SCENE & CAMERA \\
\hline C & PHOTOJOURNALISM & PRO & T & CONCOURSE & PRO \\
\hline J & PASSENGER LOADING & PRO & H & CONCOURSE & PRO \\
\hline P & SPORTS ARENA & CONSUMER & U & CHOKEPOINT & WEBCAM \\
\hline L & LUGGAGE RACK & WEBCAM & & & \\
\hline
\end{tabular}




\begin{tabular}{|c|c|c|c|c|c|c|c|c|c|c|c|c|c|c|}
\hline \multirow{3}{*}{$\begin{array}{l}\mathrm{N}=480 \\
\mathrm{ALG}\end{array}$} & \multirow{2}{*}{\multicolumn{2}{|c|}{$\begin{array}{c}\text { NUM ACTORS } 31 \\
\text { DETECTIONS }\end{array}$}} & \multicolumn{3}{|c|}{ NUM FEEDS 10} & & \multicolumn{3}{|c|}{ NUM FRAMES 40737} & \multicolumn{3}{|c|}{ NUM MINUTES 154.8} \\
\hline & & & \multicolumn{3}{|c|}{ THRESHOLD BASED AUTO WATCHLISTS } & & & & \multicolumn{6}{|c|}{ RANK BASED FORENSIC CASES } \\
\hline & NUM & $\mathrm{MIN}^{-1}$ & FNIR( & $=10$ & FNIR( & 100 & FNIR( & 000 & FNIR( & $=0)$ & FNIR(I & $=0)$ & FNIR(I & $=0)$ \\
\hline $\mathrm{A} 30 \mathrm{~V}$ & 17587 & 113.6 & 0.846 & 27 & 0.756 & 27 & 0.566 & 24 & 0.425 & 24 & 0.178 & 12 & 0.078 & 4 \\
\hline $\mathrm{A} 31 \mathrm{~V}$ & 17587 & 113.6 & 0.831 & 26 & 0.723 & 24 & 0.581 & 25 & 0.419 & 23 & 0.172 & 10 & 0.078 & 3 \\
\hline B30V & 6679 & 43.1 & 0.807 & 24 & 0.687 & 23 & 0.509 & 22 & 0.431 & 25 & 0.289 & 24 & 0.160 & 13 \\
\hline C30V & 16558 & 106.9 & 0.958 & 33 & 0.907 & 33 & 0.780 & 32 & 0.605 & 31 & 0.455 & 28 & 0.307 & 28 \\
\hline C31V & 18251 & 117.9 & 0.949 & 32 & 0.904 & 32 & 0.777 & 31 & 0.617 & 33 & 0.467 & 30 & 0.322 & 29 \\
\hline D30V & 31670 & 204.5 & 0.711 & 22 & 0.654 & 22 & 0.557 & 23 & 0.283 & 15 & 0.247 & 21 & 0.208 & 22 \\
\hline D31V & 6630 & 42.8 & 0.919 & 31 & 0.880 & 31 & 0.783 & 33 & 0.533 & 27 & 0.497 & 31 & 0.431 & 33 \\
\hline E30V & 4094 & 26.4 & 0.575 & 17 & 0.440 & 14 & 0.295 & 10 & 0.274 & 13 & 0.223 & 15 & 0.166 & 14 \\
\hline E31V & 4094 & 26.4 & 0.593 & 18 & 0.440 & 15 & 0.304 & 12 & 0.271 & 12 & 0.217 & 14 & 0.169 & 16 \\
\hline F30V & 19479 & 125.8 & 1.000 & 36 & 0.961 & 34 & 0.852 & 34 & 0.843 & 34 & 0.687 & 34 & 0.479 & 34 \\
\hline G30V & 3295 & 21.3 & 0.551 & 14 & 0.437 & 13 & 0.337 & 15 & 0.307 & 18 & 0.232 & 18 & 0.184 & 20 \\
\hline G31V & 4890 & 31.6 & 0.524 & 10 & 0.416 & 10 & 0.307 & 13 & 0.256 & 10 & 0.172 & 11 & 0.108 & 12 \\
\hline G32V & 4890 & 31.6 & 0.286 & 4 & 0.232 & 4 & 0.184 & 4 & 0.235 & 9 & 0.166 & 9 & 0.093 & 9 \\
\hline H30V & 7333 & 47.4 & 0.440 & 7 & 0.298 & 6 & 0.193 & 5 & 0.160 & 6 & 0.111 & 5 & 0.084 & 5 \\
\hline H31V & 7333 & 47.4 & 0.461 & 8 & 0.319 & 8 & 0.220 & 7 & 0.205 & 7 & 0.142 & 7 & 0.102 & 10 \\
\hline $\mathrm{H} 32 \mathrm{~V}$ & 7333 & 47.4 & 0.461 & 9 & 0.319 & 9 & 0.220 & 8 & 0.205 & 8 & 0.142 & 8 & 0.102 & 11 \\
\hline I30V & 180141 & 1163.4 & 0.398 & 6 & 0.286 & 5 & 0.199 & 6 & 0.102 & 1 & 0.057 & 1 & 0.048 & 2 \\
\hline I31V & 180141 & 1163.4 & 0.389 & 5 & 0.298 & 7 & 0.223 & 9 & 0.102 & 2 & 0.057 & 2 & 0.042 & 1 \\
\hline $\mathrm{J} 30 \mathrm{~V}$ & 4748 & 30.7 & 0.551 & 15 & 0.479 & 20 & 0.380 & 19 & 0.352 & 21 & 0.283 & 23 & 0.232 & 24 \\
\hline J31V & 4748 & 30.7 & 0.542 & 13 & 0.476 & 19 & 0.377 & 18 & 0.343 & 20 & 0.274 & 22 & 0.238 & 25 \\
\hline $\mathrm{J} 32 \mathrm{~V}$ & 4748 & 30.7 & 0.530 & 12 & 0.458 & 18 & 0.383 & 20 & 0.364 & 22 & 0.298 & 25 & 0.253 & 26 \\
\hline K30V & 17418 & 112.5 & 0.895 & 30 & 0.855 & 30 & 0.759 & 30 & 0.515 & 26 & 0.386 & 26 & 0.211 & 23 \\
\hline K31V & 4903 & 31.7 & 0.816 & 25 & 0.729 & 25 & 0.636 & 27 & 0.551 & 28 & 0.449 & 27 & 0.301 & 27 \\
\hline K32V & 3346 & 21.6 & 0.858 & 28 & 0.783 & 28 & 0.645 & 28 & 0.584 & 30 & 0.518 & 33 & 0.425 & 32 \\
\hline K33V & 2999 & 19.4 & 0.786 & 23 & 0.738 & 26 & 0.620 & 26 & 0.569 & 29 & 0.518 & 32 & 0.416 & 31 \\
\hline L30V & 8210 & 53.0 & 0.870 & 29 & 0.804 & 29 & 0.708 & 29 & 0.614 & 32 & 0.467 & 29 & 0.343 & 30 \\
\hline M30V & 8875 & 57.3 & 0.241 & 3 & 0.190 & 2 & 0.151 & 1 & 0.133 & 4 & 0.108 & 4 & 0.090 & 8 \\
\hline M31V & 8875 & 57.3 & 0.235 & 2 & 0.193 & 3 & 0.154 & 2 & 0.136 & 5 & 0.108 & 3 & 0.087 & 7 \\
\hline M32V & 8875 & 57.3 & 0.229 & 1 & 0.187 & 1 & 0.157 & 3 & 0.130 & 3 & 0.114 & 6 & 0.087 & 6 \\
\hline N30V & 3737 & 24.1 & 0.530 & 11 & 0.452 & 17 & 0.352 & 17 & 0.298 & 17 & 0.244 & 20 & 0.178 & 19 \\
\hline N31V & 3737 & 24.1 & 0.557 & 16 & 0.446 & 16 & 0.346 & 16 & 0.295 & 16 & 0.235 & 19 & 0.187 & 21 \\
\hline N32V & 3737 & 24.1 & 0.611 & 19 & 0.428 & 12 & 0.304 & 11 & 0.262 & 11 & 0.208 & 13 & 0.175 & 17 \\
\hline N33V & 3737 & 24.1 & 0.630 & 20 & 0.422 & 11 & 0.313 & 14 & 0.277 & 14 & 0.226 & 16 & 0.169 & 15 \\
\hline Q30V & 5773 & 37.3 & 0.997 & 34 & 0.997 & 36 & 0.967 & 36 & 0.940 & 36 & 0.852 & 36 & 0.699 & 36 \\
\hline Q31V & 5773 & 37.3 & 0.997 & 35 & 0.985 & 35 & 0.928 & 35 & 0.898 & 35 & 0.804 & 35 & 0.599 & 35 \\
\hline R30V & 10518 & 67.9 & 0.699 & 21 & 0.566 & 21 & 0.425 & 21 & 0.316 & 19 & 0.226 & 17 & 0.175 & 18 \\
\hline
\end{tabular}

Table 27: For the DATASET T: TRAVEL WALKWAY installation, with 480 subjects enrolled with a frontal still, the values are detection counts summed over 10 cameras, the rate over all cameras, identification-mode $F N I R(T)$ for each algorithm at three different decision thresholds corresponding to false positive counts of 10,100,1000, and investigation-mode FNIR(R) for ranks 1, 5, 20. Each value is accompanied by an integer ranking across all algorithms. The shading indicates the most important metric to watchlist applications. 10 cameras were used. Their frames rates varied from $1.5 \mathrm{fps}$ to $20 \mathrm{fps}$. The detections are summed over all of them. The accuracy values are aggregated over all sightings of all subjects in the field of view of those cameras. NB: This dataset is not sequestered it has been made available to some developers. Their ability to tune and train on this data may mean that accuracy values may be optimistic.

\subsection{DATASET T: TRAVEL WALKWAY Surveillance}

Experimental design: The dataset is composed of videos collected in a transit terminal/passenger environment. As with Dataset $\mathrm{H}$, actors and members of the general public walk underneath ceiling mounted cameras. Extracts from dataset has been made available to certain face recognition algorithm developers. Thus, the dataset is not sequestered, and should not therefore be relied upon when comparing algorithms.

Mated scores are computed by searching 329 video clips against an enrolled dataset of still face images of subjects known to be in the search videos. The size of the enrollment dataset was 480 or 4800 . The enrollment database is extended to these sizes by adding high quality frontal portrait photographs from a disjoint background population.

Nonmated scores are computed by replacing the gallery, which normally contains frontal images of known actors, with the global nonmated enrollment dataset.

Results: Detection and recognition error rates are tabulated in Tables 27 and 28. Notably the detection counts are 2-5

\begin{tabular}{|l|l|l|l|}
\hline \multicolumn{5}{|c|}{ PARTICIPANT KEY } \\
\hline $\mathrm{A}=$ DIGITAL BARRIERS & $\mathrm{E}=$ NEUROTECHNOLOGY & $\mathrm{I}=$ EYEDEA & $\mathrm{M}=$ NEC \\
\hline $\mathrm{B}=$ HBINNO & $\mathrm{F}=$ VAPPLICA & $\mathrm{J}=$ HISIGN & $\mathrm{N}=$ TOSHIBA \\
\hline $\mathrm{C}=$ VIGILANT & $\mathrm{G}=$ MORPHO & $\mathrm{K}=$ COGNITEC & $\mathrm{Q}=$ IMAGUS \\
\hline $\mathrm{D}=$ AYONIX & $\mathrm{H}=$ 3M COGENT & $\mathrm{L}=$ CYBEREXTRUDER & $\mathrm{R}=$ RANK ONE \\
\hline
\end{tabular}

\begin{tabular}{|l|l|l|l|l|l|}
\hline SET & SCENE & CAMERA & SET & SCENE & CAMERA \\
\hline C & PHOTOJOURNALISM & PRO & T & CONCOURSE & PRO \\
\hline J & PASSENGER LOADING & PRO & H & CONCOURSE & PRO \\
\hline P & SPORTS ARENA & CONSUMER & U & CHOKEPOINT & WEBCAM \\
\hline L & LUGGAGE RACK & WEBCAM & & & \\
\hline
\end{tabular}




\begin{tabular}{|c|c|c|c|c|c|c|c|c|c|c|c|c|c|c|}
\hline \multirow{3}{*}{$\begin{array}{l}\mathrm{N}=4800 \\
\text { ALG }\end{array}$} & \multirow{2}{*}{\multicolumn{2}{|c|}{$\begin{array}{c}\text { NUM ACTORS } 31 \\
\text { DETECTIONS }\end{array}$}} & \multicolumn{3}{|c|}{ NUM FEEDS 10} & \multicolumn{3}{|c|}{ NUM CLIPS 329} & \multicolumn{3}{|c|}{ NUM FRAMES 40737} & \multicolumn{3}{|c|}{ NUM MINUTES 154.8} \\
\hline & & & \multicolumn{6}{|c|}{ THRESHOLD BASED AUTO WATCHLISTS } & \multicolumn{6}{|c|}{ RANK BASED FORENSIC CASES } \\
\hline & NUM & $\mathrm{MIN}^{-1}$ & FNIR( & $=10$ & FNIR( & 00 & FNIR(1 & 000 & FNIR(I & $=0)$ & FNIR(I & $=0)$ & FNIR( & $=0)$ \\
\hline A30V & 17587 & 113.6 & 0.919 & 26 & 0.834 & 23 & 0.678 & 24 & 0.581 & 24 & 0.401 & 23 & 0.256 & 17 \\
\hline A31V & 17587 & 113.6 & 1.000 & 35 & 1.000 & 35 & 1.000 & 36 & 1.000 & 36 & 1.000 & 36 & 1.000 & 36 \\
\hline B30V & 6670 & 43.1 & 0.837 & 22 & 0.747 & 22 & 0.596 & 22 & 0.518 & 23 & 0.422 & 24 & 0.331 & 24 \\
\hline $\mathrm{C} 30 \mathrm{~V}$ & 16558 & 106.9 & 0.961 & 29 & 0.946 & 29 & 0.852 & 29 & 0.660 & 27 & 0.536 & 26 & 0.440 & 26 \\
\hline C31V & 18251 & 117.9 & 0.967 & 30 & 0.949 & 31 & 0.858 & 31 & 0.681 & 31 & 0.536 & 25 & 0.431 & 25 \\
\hline D30V & 31643 & 204.4 & 0.795 & 20 & 0.699 & 21 & 0.608 & 23 & 0.377 & 18 & 0.316 & 18 & 0.283 & 20 \\
\hline D31V & 6622 & 42.8 & 0.961 & 28 & 0.934 & 28 & 0.855 & 30 & 0.593 & 25 & 0.578 & 28 & 0.563 & 32 \\
\hline E30V & 4094 & 26.4 & 0.714 & 18 & 0.542 & 17 & 0.401 & 14 & 0.325 & 13 & 0.259 & 12 & 0.223 & 11 \\
\hline E31V & 4094 & 26.4 & 0.994 & 32 & 0.946 & 30 & 0.470 & 19 & 0.316 & 12 & 0.247 & 11 & 0.226 & 12 \\
\hline F30V & 19487 & 125.8 & 1.000 & 36 & 1.000 & 36 & 0.928 & 33 & 0.904 & 33 & 0.825 & 33 & 0.693 & 33 \\
\hline G30V & 3311 & 21.4 & 0.642 & 16 & 0.503 & 12 & 0.386 & 13 & 0.355 & 16 & 0.283 & 14 & 0.238 & 13 \\
\hline G31V & 4858 & 31.4 & 0.714 & 19 & 0.545 & 18 & 0.410 & 15 & 0.307 & 11 & 0.238 & 10 & 0.190 & 10 \\
\hline G32V & 4890 & 31.6 & 0.608 & 12 & 0.512 & 14 & 0.352 & 10 & 0.286 & 9 & 0.223 & 9 & 0.175 & 9 \\
\hline H30V & 7333 & 47.4 & 0.262 & 1 & 0.229 & 3 & 0.223 & 4 & 0.235 & 8 & 0.196 & 8 & 0.166 & 6 \\
\hline H31V & 7333 & 47.4 & 0.452 & 5 & 0.334 & 5 & 0.280 & 5 & 0.235 & 6 & 0.193 & 7 & 0.169 & 7 \\
\hline $\mathrm{H} 32 \mathrm{~V}$ & 7333 & 47.4 & 0.452 & 6 & 0.334 & 6 & 0.280 & 6 & 0.235 & 7 & 0.190 & 6 & 0.169 & 8 \\
\hline $\mathrm{I} 30 \mathrm{~V}$ & 180141 & 1163.4 & 0.491 & 7 & 0.370 & 7 & 0.286 & 7 & 0.142 & 1 & 0.093 & 2 & 0.072 & 2 \\
\hline I31V & 180141 & 1163.4 & 0.548 & 9 & 0.410 & 8 & 0.292 & 8 & 0.145 & 2 & 0.087 & 1 & 0.060 & 1 \\
\hline J30V & 4748 & 30.7 & 0.633 & 14 & 0.536 & 16 & 0.473 & 20 & 0.413 & 21 & 0.355 & 21 & 0.304 & 22 \\
\hline $\mathrm{J} 31 \mathrm{~V}$ & 4748 & 30.7 & 0.645 & 17 & 0.524 & 15 & 0.455 & 18 & 0.407 & 20 & 0.349 & 20 & 0.304 & 21 \\
\hline $\mathrm{J} 32 \mathrm{~V}$ & 4748 & 30.7 & 0.620 & 13 & 0.545 & 19 & 0.449 & 17 & 0.419 & 22 & 0.370 & 22 & 0.322 & 23 \\
\hline K30V & 17418 & 112.5 & 0.967 & 31 & 0.955 & 32 & 0.913 & 32 & 0.657 & 26 & 0.566 & 27 & 0.470 & 27 \\
\hline K31V & 4903 & 31.7 & 0.892 & 23 & 0.877 & 25 & 0.801 & 27 & 0.672 & 28 & 0.590 & 29 & 0.500 & 28 \\
\hline K32V & 3346 & 21.6 & 0.934 & 27 & 0.892 & 27 & 0.801 & 28 & 0.678 & 30 & 0.627 & 31 & 0.548 & 30 \\
\hline K33V & 2999 & 19.4 & 0.904 & 24 & 0.855 & 24 & 0.783 & 25 & 0.675 & 29 & 0.593 & 30 & 0.551 & 31 \\
\hline L30V & 8202 & 53.0 & 0.907 & 25 & 0.880 & 26 & 0.789 & 26 & 0.738 & 32 & 0.648 & 32 & 0.545 & 29 \\
\hline M30V & 8875 & 57.3 & 0.268 & 2 & 0.226 & 2 & 0.175 & 1 & 0.157 & 5 & 0.130 & 4 & 0.114 & 3 \\
\hline M31V & 8875 & 57.3 & 0.274 & 3 & 0.232 & 4 & 0.181 & 3 & 0.157 & 4 & 0.133 & 5 & 0.117 & 4 \\
\hline M32V & 8875 & 57.3 & 0.298 & 4 & 0.217 & 1 & 0.181 & 2 & 0.151 & 3 & 0.127 & 3 & 0.123 & 5 \\
\hline N30V & 3737 & 24.1 & 0.554 & 10 & 0.449 & 10 & 0.361 & 11 & 0.331 & 14 & 0.292 & 16 & 0.256 & 18 \\
\hline N31V & 3737 & 24.1 & 0.633 & 15 & 0.506 & 13 & 0.413 & 16 & 0.377 & 19 & 0.319 & 19 & 0.271 & 19 \\
\hline N32V & 3737 & 24.1 & 0.527 & 8 & 0.434 & 9 & 0.349 & 9 & 0.307 & 10 & 0.277 & 13 & 0.244 & 14 \\
\hline N33V & 3737 & 24.1 & 0.590 & 11 & 0.467 & 11 & 0.373 & 12 & 0.346 & 15 & 0.286 & 15 & 0.253 & 16 \\
\hline Q30V & 5773 & 37.3 & 1.000 & 34 & 1.000 & 34 & 0.997 & 35 & 0.964 & 35 & 0.934 & 35 & 0.867 & 35 \\
\hline Q31V & 5773 & 37.3 & 0.997 & 33 & 0.994 & 33 & 0.970 & 34 & 0.946 & 34 & 0.886 & 34 & 0.786 & 34 \\
\hline R30V & 10517 & 67.9 & 0.822 & 21 & 0.663 & 20 & 0.491 & 21 & 0.367 & 17 & 0.307 & 17 & 0.244 & 15 \\
\hline
\end{tabular}

Table 28: For the DATASET T: TRAVEL WALKWAY installation, with 4800 subjects enrolled with a frontal still, the values are detection counts summed over 10 cameras, the rate over all cameras, identification-mode FNIR(T) for each algorithm at three different decision thresholds corresponding to false positive counts of 10,100,1000, and investigation-mode FNIR(R) for ranks 1, 5, 20. Each value is accompanied by an integer ranking across all algorithms. The shading indicates the most important metric to watchlist applications. 10 cameras were used. Their frames rates varied from $1.5 \mathrm{fps}$ to $20 \mathrm{fps}$. The detections are summed over all of them. The accuracy values are aggregated over all sightings of all subjects in the field of view of those cameras. NB: This dataset is not sequestered it has been made available to some developers. Their ability to tune and train on this data may mean that accuracy values may be optimistic.

\begin{tabular}{|c|c|c|c|c|c|c|c|c|c|}
\hline \multicolumn{4}{|c|}{ PARTICIPANT KEY } & SET & SCENE & CAMERA & SET & SCENE & CAMERA \\
\hline $\mathrm{A}=$ DIGITAL BARRIERS & $\mathrm{E}=$ NEUROTECHNOLOGY & $I=$ EYEDEA & $M=N E C$ & C & PHOTOJOURNALISM & PRO & $\mathrm{T}$ & CONCOURSE & PRO \\
\hline $\mathrm{B}=\mathrm{HBINNO}$ & $\mathrm{F}=\mathrm{VAPPLICA}$ & $\mathrm{J}=\mathrm{HISIGN}$ & $\mathrm{N}=$ TOSHIBA & $\mathrm{J}$ & PASSENGER LOADING & PRO & $\mathrm{H}$ & CONCOURSE & PRO \\
\hline $\mathrm{C}=$ VIGILANT & $\mathrm{G}=\mathrm{MORPHO}$ & $\mathrm{K}=$ COGNITEC & $\mathrm{Q}=$ IMAGUS & $\mathrm{P}$ & SPORTS ARENA & CONSUMER & $\mathrm{U}$ & CHOKEPOINT & WEBCAM \\
\hline $\mathrm{D}=\mathrm{AYONIX}$ & $\mathrm{H}=3 \mathrm{M}$ COGENT & $\mathrm{L}=$ CYBEREXTRUDER & $\mathrm{R}=\mathrm{RANK}$ ONE & $\mathrm{L}$ & LUGGAGE RACK & WEBCAM & & & \\
\hline
\end{tabular}


times higher than with Dataset $\mathrm{H}$, depending on algorithm, and this reflects the somewhat wider fields of view and denser crowds in T vs. H. There is one exception to this, algorithms I3xV, both report enormous numbers of detections, almost 100 times more than with Dataset $\mathrm{H}$. We have no explanation for this anomalous behavior, but note that the same algorithms produce the best rank-based miss rates. While the I algorithms are competitive on other datasets, it is possible that by producing many templates, the search accuracy is artificially improved by sheer volume of searches, some of which fortuitously place the correct actor at rank 1 . This applies with both $\mathrm{N}=480$ and 4800 . This observation is consistent with I algorithms having relatively lower performance when the threshold is raised to produce only small numbers of false positives. Whether the developers of the I algorithms experimented with Dataset $\mathrm{T}$ is not known.

While the $\mathrm{M}$ and I algorithms give similar rank one miss rates for Datasets $\mathrm{T}$ and $\mathrm{H}$, other algorithms give generally higher rate on the $\mathrm{T}$ data. The cause of this is unknown, but may be associated with higher crowd densities. The highthreshold FNIR(N, L, T) rates are not easily comparable because the thresholds set to achieve, for example, NFP(T) $=100$ are different given that the datasets differ in the total number of people appearing in the videos. The Dataset $\mathrm{H}$ error rates are generally lower, and again we'd like to attribute this to lower volumes (travelers per minute) with Dataset $\mathrm{H}$, but a large number of other factors come into play. The only solid conclusion is that while Datasets $\mathrm{T}$ and $\mathrm{H}$ are both nominally professional installations of video surveillance cameras, the observed differences in identification miss rates are essentially a measure of uncertainty such that a deployer cannot know a priori precisely how well face recognition will work in their environment.

\begin{tabular}{|l|l|l|l|}
\hline \multicolumn{4}{|c|}{ PARTICIPANT KEY } \\
\hline $\mathrm{A}=$ DIGITAL BARRIERS & $\mathrm{E}=$ NEUROTECHNOLOGY & $\mathrm{I}=$ EYEDEA & $\mathrm{M}=$ NEC \\
\hline $\mathrm{B}=$ HBINNO & $\mathrm{F}=$ VAPPLICA & $\mathrm{J}=$ HISIGN & $\mathrm{N}=$ TOSHIBA \\
\hline $\mathrm{C}=$ VIGILANT & $\mathrm{G}=$ MORPHO & $\mathrm{K}=$ COGNITEC & $\mathrm{Q}=$ IMAGUS \\
\hline $\mathrm{D}=$ AYONIX & $\mathrm{H}=$ 3M COGENT & $\mathrm{L}=$ CYBEREXTRUDER & $\mathrm{R}=$ RANK ONE \\
\hline
\end{tabular}

\begin{tabular}{|l|l|l|l|l|l|}
\hline SET & SCENE & CAMERA & SET & SCENE & CAMERA \\
\hline C & PHOTOJOURNALISM & PRO & T & CONCOURSE & PRO \\
\hline J & PASSENGER LOADING & PRO & H & CONCOURSE & PRO \\
\hline P & SPORTS ARENA & CONSUMER & U & CHOKEPOINT & WEBCAM \\
\hline L & LUGGAGE RACK & WEBCAM & & & \\
\hline
\end{tabular}




\begin{tabular}{|l|l|}
\hline Property & Value \\
\hline Cameras & Many, mostly professional still + television \\
\hline Camera mounting & Handheld or fixed mount \\
\hline Camera height & Near head height \\
\hline Camera declination to face & Adverse yaw, only modest pitch \\
\hline Frame rate & Variable, usually $\geq 24 \mathrm{sec}^{-1}$ \\
\hline Width & Variable \\
\hline Height & Variable \\
\hline
\end{tabular}

Table 29: Key imaging properties for DATASET C: PHOTOJOURNALISM results.

\subsection{DATASET C: PHOTOJOURNALISM}

Overview: This section documents two experiments, one video-to-still (as in the rest of this report) and one video-to-video. Both experiments use imagery comprised of unconstrained photographs and videos of celebrities, actors, politicians, and diplomats. The images are quite different in character compared to the others used in this report. First, they were not collected with any notion that face recognition would be applied. Second, as they were mostly acquired by professional photo journalists, they are quality-biased, in the sense that they have survived a selection process in which, mostly, they do not exhibit poor focus and poor exposure. Instead, the images are selected to be engaging to a human viewer. Thus, neutral expressions and fully frontal views are not the norm. Some faces are partially occluded, and there are wide variations in head pose and expression. While the term "in the wild" has been used to describe such data [24], it is a misnomer in the sense that professional photographers collect better constrained data than entirely amateur "wild" and un-constrained data. A slightly better description might be "in the limelight"! Note that the imagery used here was not selected on the basis that a face detector found the faces and selected it for inclusion. Note also that the imagery is in the public domain, and could, in-principle have been used in training the algorithms submitted to FIVE. The still images and video frames from this dataset have recently been released $[26]^{15}$.

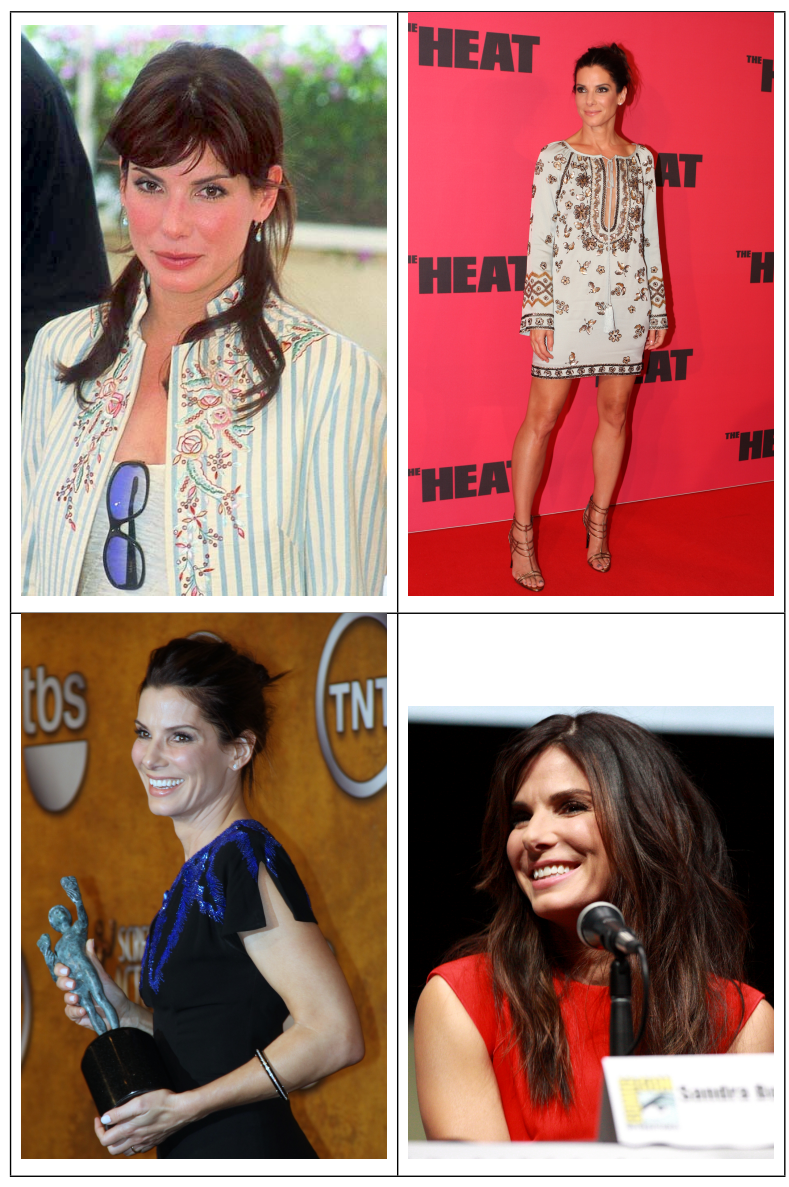

Figure 37: DATASET C: PHOTOJOURNALISM : Examples of "in the wild" photojournalism stills. ${ }^{* *}$ The images in this figure are of celebrities and politicians. They are freely available on the internet under Creative Commons licenses.

The type of equipment is not known. Given the photojournalism origins of most of the data, we can assume that the cameras were of professional grade.

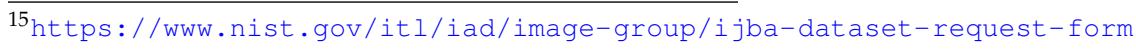

\begin{tabular}{|l|l|l|l|}
\hline \multicolumn{4}{|c|}{ PARTICIPANT KEY } \\
\hline A = DIGITAL BARRIERS & E = NEUROTECHNOLOGY & I = EYEDEA & M = NEC \\
\hline B = HBINNO & F = VAPPLICA & J = HISIGN & N = TOSHIBA \\
\hline C = VIGILANT & G = MORPHO & K = COGNITEC & Q = IMAGUS \\
\hline D = AYONIX & H = 3M COGENT & L = CYBEREXTRUDER & R = RANK ONE \\
\hline
\end{tabular}

\begin{tabular}{|l|l|l|l|l|l|}
\hline SET & SCENE & CAMERA & SET & SCENE & CAMERA \\
\hline C & PHOTOJOURNALISM & PRO & T & CONCOURSE & PRO \\
\hline J & PASSENGER LOADING & PRO & H & CONCOURSE & PRO \\
\hline P & SPORTS ARENA & CONSUMER & U & CHOKEPOINT & WEBCAM \\
\hline L & LUGGAGE RACK & WEBCAM & & & \\
\hline
\end{tabular}



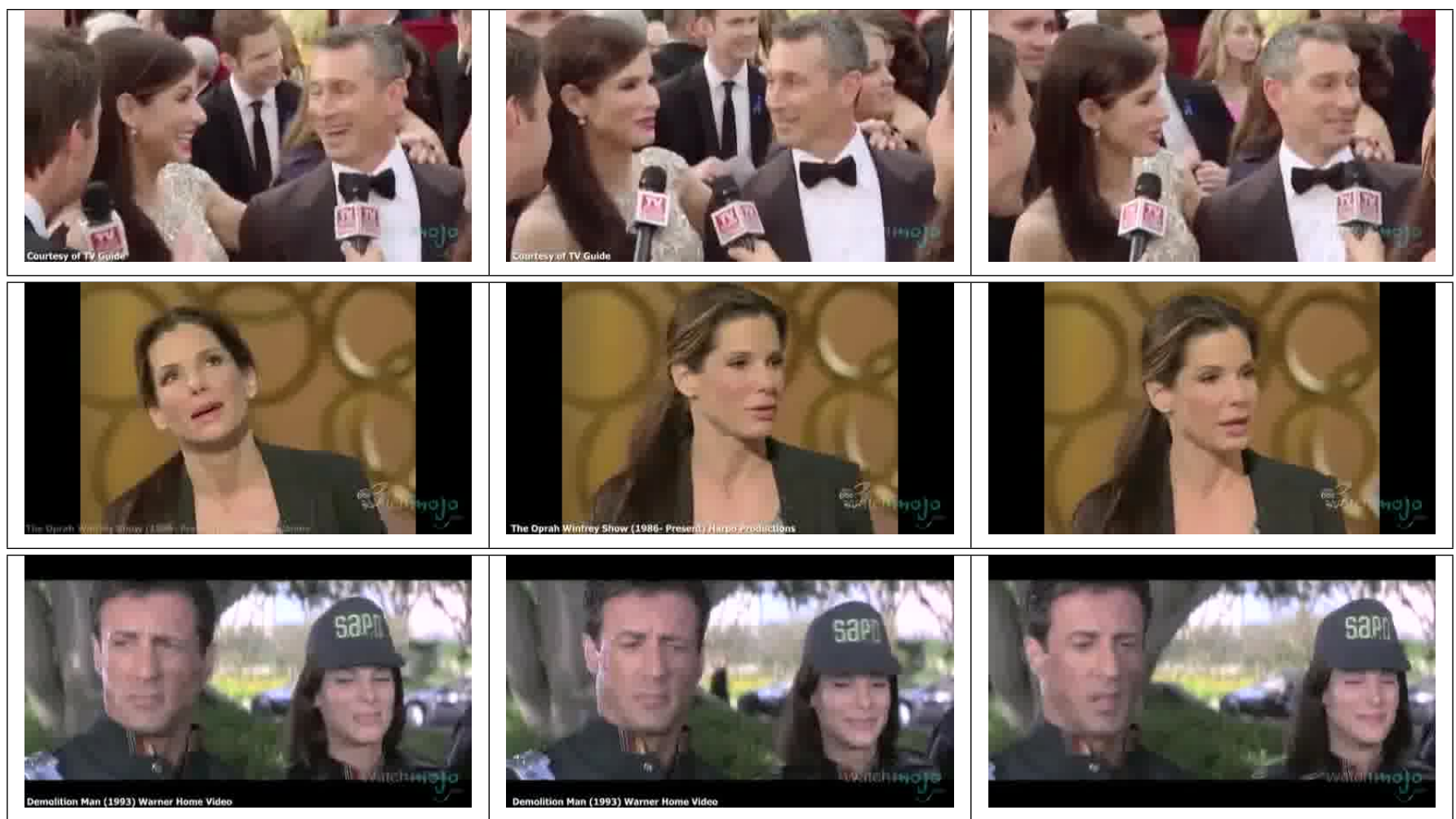

Figure 38: DATASET C: PHOTOJOURNALISM : Examples of "in the wild" photojournalism video clips. ${ }^{* *}$ The video frames in this figure are taken from videos of celebrities and politicians that are available on the internet under Creative Commons licenses.

Experimental design: The individuals are often giving speeches or are being interviewed. There are sometimes many other people in the scene - see, for example, Figure 38. Two experiments were conducted.

In the first video-to-still experiment, a common set of 7194 videos were searched against two still galleries, one of size 940 (S1), and the other of size 930 (S2) persons. Both galleries are composed of unconstrained face photographs, one per subject, examples of which are shown in Figure 37. The probeset contains both mated and nonmated search videos: A subset denoted P1 forms the mated searches for S1 and the non-mated searches for S2. The disjoint subset P2 forms the nonmated searches for S1 and mated searches for S2. Example videos appear in Figure 38.

In the second video-to-video experiment, 1356 mated video clips are searched against templates extracted from each of 393 gallery video clips. Enrollment of this kind generally produces more templates than there are videos, because multiple faces appear in some videos. As all searches contain at least one face known to be in the gallery, this experiment is "closed universe" and is therefore atypical operationally.

Key experimental design details are summarized in Table 30.

\begin{tabular}{|c|c|c|c|}
\hline \multicolumn{4}{|c|}{ PARTICIPANT KEY } \\
\hline $\mathrm{A}=$ DIGITAL BARRIERS & $\mathrm{E}=\mathrm{NEUROTECHNOLOGY}$ & $I=$ EYEDEA & $M=N E C$ \\
\hline $\mathrm{B}=\mathrm{HBINNO}$ & $\mathrm{F}=$ VAPPLICA & $\mathrm{J}=\mathrm{HISIGN}$ & $\mathrm{N}=$ TOSHIBA \\
\hline $\mathrm{C}=$ VIGILANT & $G=M O R P H O$ & $\mathrm{~K}=$ COGNITEC & $Q=$ IMAGUS \\
\hline $\mathrm{D}=\mathrm{AYONIX}$ & $\mathrm{H}=3 \mathrm{M}$ COGENT & $\mathrm{L}=$ CYBEREXTRUDER & $\mathrm{R}=\mathrm{RANK}$ ONE \\
\hline
\end{tabular}

\begin{tabular}{|l|l|l|l|l|l|}
\hline SET & SCENE & CAMERA & SET & SCENE & CAMERA \\
\hline C & PHOTOJOURNALISM & PRO & T & CONCOURSE & PRO \\
\hline J & PASSENGER LOADING & PRO & H & CONCOURSE & PRO \\
\hline P & SPORTS ARENA & CONSUMER & U & CHOKEPOINT & WEBCAM \\
\hline L & LUGGAGE RACK & WEBCAM & & & \\
\hline
\end{tabular}




\begin{tabular}{|l|l|}
\hline Quantity & Value or description \\
\hline \hline Mode & Video search to video enrollment \\
\hline Number of enrolled subjects & 393 \\
\hline Number of enrolled videos & 393 \\
\hline Number of enrolled stills & 0 \\
\hline Number of actors & 393 \\
\hline Number of non-actors & Many \\
\hline Number of search clips actors & 1356 \\
\hline Number of search clips no actors & 0 \\
\hline Video duration with actors (minutes) & 699 \\
\hline Video duration no actors & 0 \\
\hline Gallery video duration (frames) & Variable: 433 median, 765 mean \\
\hline Probe video duration (frames) & Variable: 460 median, 847 mean \\
\hline Properties of enrolled videos & Unconstrained mostly photojournalism \\
\hline FNIR estimation & Actors present video vs. enrolled gallery \\
\hline FPIR estimation & Not done \\
\hline \hline Mode & Video search to still enrollment \\
\hline Number of enrolled subjects & Two disjoint galleries, S1-940, S2-930 \\
\hline Number of enrolled videos & 0 \\
\hline Number of enrolled stills & Multiple per subject \\
\hline Number of actors & 1870 \\
\hline Number of search clips & 7195 about half actors, half non-actors \\
\hline Properties of enrolled stills & Unconstrained mostly photojournalism \\
\hline FNIR estimation & Actors present video vs. enrolled gallery: P1-S1, P2-S2 \\
\hline FPIR estimation & Actors absent video vs. same enrolled gallery: P1-S2, P2-S1 \\
\hline Probe video frame rate (per second) & Variable, many 24 \\
\hline Probe video total duration (frames) & 4493 284 \\
\hline Probe video clip duration (frames) & Variable: 390 median, 625 mean \\
\hline \hline & Factors in common \\
\hline Candidate list length & 20 \\
\hline Subject motion & Often on podium or seated facing journalist \\
\hline Number of persons in FOV & Variable, typically 1, 2, few \\
\hline Number of cameras & Many, often professional \\
\hline Video ground truth & Style A: See Figure 6 \\
\hline
\end{tabular}

Table 30: Key experimental design for the DATASET C: PHOTOJOURNALISM results.

\begin{tabular}{|c|c|c|c|c|c|c|c|c|c|}
\hline \multicolumn{4}{|c|}{ PARTICIPANT KEY } & \multirow{2}{*}{\begin{tabular}{|l|} 
SET \\
$\mathrm{C}$ \\
\end{tabular}} & SCENE & \multirow{2}{*}{\begin{tabular}{|l|} 
CAMERA \\
PRO
\end{tabular}} & \multirow{2}{*}{\begin{tabular}{|l|} 
SET \\
$\mathrm{T}$ \\
\end{tabular}} & \multirow{2}{*}{\begin{tabular}{|l|} 
SCENE \\
CONCOURSE \\
\end{tabular}} & \multirow{2}{*}{\begin{tabular}{|l} 
CAMERA \\
PRO
\end{tabular}} \\
\hline $\mathrm{A}=$ DIGITAL BARRIERS & $\mathrm{E}=$ NEUROTECHNOLOGY & $\mathrm{I}$ = EYEDEA & $M=N E C$ & & PHOTOJOURNALISM & & & & \\
\hline $\mathrm{C}=$ VIGILANT & $\mathrm{G}=\mathrm{MORPHO}$ & $\mathrm{K}=$ COGNITEC & $Q=$ IMAGUS & $\mathrm{P}$ & SPORTS ARENA & CONSUMER & $\mathrm{U}$ & CHOKEPOINT & WEBCAM \\
\hline $\mathrm{D}=$ AYONIX & $\mathrm{H}=3 \mathrm{M}$ COGENT & $\mathrm{L}=$ CYBEREXTRUDER & $\mathrm{R}=\mathrm{RANK}$ ONE & $\mathrm{L}$ & LUGGAGE RACK & WEBCAM & & & \\
\hline
\end{tabular}




\begin{tabular}{|c|c|c|c|c|c|c|c|c|c|c|c|c|c|}
\hline \multirow{3}{*}{$\begin{array}{l}\mathrm{N}=930 \mathrm{OR} \\
\mathrm{N}=940 \\
\text { ALG }\end{array}$} & \multicolumn{2}{|c|}{ NUM ACTORS 1870} & \multicolumn{2}{|c|}{ NUM FEEDS 7194} & \multicolumn{3}{|c|}{ NUM CLIPS 7194} & \multicolumn{3}{|c|}{ NUM FRAMES 4493284} & \multicolumn{3}{|c|}{ NUM MINUTES 2781.5} \\
\hline & \multicolumn{2}{|c|}{ DETECTIONS } & \multicolumn{5}{|c|}{ THRESHOLD BASED IDENTIFICATION } & \multicolumn{6}{|c|}{ RANK BASED INVESTIGATION } \\
\hline & NUM & $\begin{array}{l}\text { FNIR(T } \\
\text { FPIR(T }\end{array}$ & $\begin{array}{l}\mathrm{FP}(\mathrm{T})=1, \\
0.0001\end{array}$ & $\begin{array}{l}\text { FNIR( } \\
\text { FPIR(T }\end{array}$ & 10 & $\begin{array}{l}\text { FNIR(T } \\
\text { FPIR(T }\end{array}$ & 00 & FNIR(I & $=0)$ & FNIR(I & $\Gamma=0)$ & FNIR( & $=0)$ \\
\hline $\mathrm{A} 30 \mathrm{~V}$ & 431026 & 0.993 & 26 & 0.975 & 26 & 0.955 & 27 & 0.816 & 28 & 0.696 & 28 & 0.138 & 10 \\
\hline A31V & 431026 & 0.992 & 23 & 0.988 & 29 & 0.980 & 29 & 0.816 & 27 & 0.696 & 27 & 0.138 & 9 \\
\hline B30V & 52954 & 0.975 & 3 & 0.936 & 14 & 0.898 & 22 & 0.706 & 25 & 0.571 & 25 & 0.325 & 31 \\
\hline C30V & 297740 & 0.994 & 29 & 0.992 & 31 & 0.985 & 31 & 0.913 & 31 & 0.798 & 31 & 0.233 & 28 \\
\hline C31V & 341668 & 0.993 & 28 & 0.991 & 30 & 0.985 & 30 & 0.916 & 32 & 0.798 & 32 & 0.228 & 27 \\
\hline D30V & 219672 & 0.998 & 31 & 0.998 & 33 & 0.998 & 33 & 0.954 & 35 & 0.810 & 33 & 0.426 & 34 \\
\hline D31V & 91868 & 0.982 & 5 & 0.975 & 27 & 0.964 & 28 & 0.879 & 29 & 0.697 & 29 & 0.395 & 32 \\
\hline E30V & 53578 & 0.975 & 4 & 0.954 & 23 & 0.873 & 19 & 0.550 & 10 & 0.412 & 12 & 0.215 & 25 \\
\hline E31V & 53540 & 0.971 & 1 & 0.960 & 24 & 0.932 & 24 & 0.575 & 16 & 0.433 & 16 & 0.216 & 26 \\
\hline F30V & 507530 & 1.000 & 36 & 1.000 & 36 & 1.000 & 36 & 0.994 & 36 & 0.960 & 36 & 0.476 & 36 \\
\hline G30V & 44229 & 0.992 & 24 & 0.925 & 12 & 0.841 & 16 & 0.621 & 22 & 0.476 & 22 & 0.260 & 29 \\
\hline G31V & 46406 & 0.992 & 22 & 0.914 & 6 & 0.705 & 2 & 0.445 & 5 & 0.307 & 4 & 0.135 & 8 \\
\hline G32V & 46191 & 0.991 & 21 & 0.841 & 1 & 0.624 & 1 & 0.359 & 1 & 0.245 & 1 & 0.108 & 6 \\
\hline H30V & 80856 & 0.999 & 33 & 0.954 & 22 & 0.849 & 18 & 0.596 & 19 & 0.465 & 21 & 0.190 & 18 \\
\hline H31V & 80856 & 0.991 & 19 & 0.951 & 20 & 0.815 & 11 & 0.588 & 17 & 0.459 & 19 & 0.186 & 16 \\
\hline H32V & 80856 & 0.991 & 20 & 0.951 & 21 & 0.822 & 14 & 0.593 & 18 & 0.465 & 20 & 0.190 & 19 \\
\hline $\mathrm{I} 30 \mathrm{~V}$ & 131712 & 0.993 & 25 & 0.922 & 11 & 0.797 & 7 & 0.494 & 6 & 0.346 & 6 & 0.095 & 4 \\
\hline I31V & 131712 & 0.993 & 27 & 0.983 & 28 & 0.937 & 25 & 0.561 & 12 & 0.401 & 10 & 0.099 & 5 \\
\hline $\mathrm{J} 30 \mathrm{~V}$ & 46554 & 0.990 & 18 & 0.910 & 4 & 0.813 & 10 & 0.570 & 15 & 0.426 & 15 & 0.213 & 24 \\
\hline $\mathrm{J} 31 \mathrm{~V}$ & 46554 & 0.989 & 17 & 0.915 & 7 & 0.803 & 8 & 0.566 & 13 & 0.422 & 13 & 0.210 & 23 \\
\hline $\mathrm{J} 32 \mathrm{~V}$ & 46554 & 0.986 & 12 & 0.941 & 16 & 0.817 & 12 & 0.557 & 11 & 0.409 & 11 & 0.188 & 17 \\
\hline K30V & 219018 & 0.988 & 14 & 0.968 & 25 & 0.943 & 26 & 0.712 & 26 & 0.587 & 26 & 0.164 & 13 \\
\hline K31V & 82280 & 0.983 & 6 & 0.938 & 15 & 0.896 & 21 & 0.639 & 23 & 0.493 & 24 & 0.195 & 20 \\
\hline K32V & 53494 & 0.986 & 11 & 0.943 & 18 & 0.900 & 23 & 0.605 & 21 & 0.459 & 18 & 0.207 & 22 \\
\hline K33V & 49348 & 0.985 & 10 & 0.935 & 13 & 0.886 & 20 & 0.601 & 20 & 0.441 & 17 & 0.201 & 21 \\
\hline L30V & 159850 & 0.999 & 32 & 0.997 & 32 & 0.993 & 32 & 0.891 & 30 & 0.766 & 30 & 0.276 & 30 \\
\hline M30V & 120034 & 0.987 & 13 & 0.922 & 10 & 0.713 & 4 & 0.419 & 2 & 0.305 & 2 & 0.065 & 3 \\
\hline M31V & 120034 & 0.989 & 16 & 0.911 & 5 & 0.722 & 5 & 0.425 & 3 & 0.307 & 5 & 0.064 & 1 \\
\hline M32V & 120034 & 0.984 & 9 & 0.943 & 17 & 0.710 & 3 & 0.426 & 4 & 0.305 & 3 & 0.064 & 2 \\
\hline N30V & 50806 & 0.983 & 8 & 0.902 & 3 & 0.777 & 6 & 0.514 & 7 & 0.387 & 8 & 0.167 & 14 \\
\hline N31V & 50806 & 0.988 & 15 & 0.920 & 9 & 0.824 & 15 & 0.537 & 9 & 0.400 & 9 & 0.146 & 12 \\
\hline N32V & 50806 & 0.994 & 30 & 0.891 & 2 & 0.809 & 9 & 0.568 & 14 & 0.423 & 14 & 0.182 & 15 \\
\hline N33V & 50806 & 0.973 & 2 & 0.916 & 8 & 0.821 & 13 & 0.529 & 8 & 0.381 & 7 & 0.141 & 11 \\
\hline Q30V & 125234 & 1.000 & 35 & 1.000 & 35 & 1.000 & 35 & 0.930 & 34 & 0.830 & 34 & 0.474 & 35 \\
\hline Q31V & 125234 & 1.000 & 34 & 1.000 & 34 & 0.999 & 34 & 0.925 & 33 & 0.831 & 35 & 0.412 & 33 \\
\hline R30V & 111832 & 0.983 & 7 & 0.945 & 19 & 0.845 & 17 & 0.641 & 24 & 0.477 & 23 & 0.125 & 7 \\
\hline
\end{tabular}

Table 31: For the DATASET C: PHOTOJOURNALISM installation, with 1870 subjects enrolled with one or more unconstrained stills, the values are identification-mode $\mathrm{FNIR}(\mathrm{N}, \mathrm{L}, \mathrm{T})$ for each algorithm at three different decision thresholds corresponding to false positive counts of 1,10,100, and investigation-mode $\operatorname{FNIR}(\mathrm{N}, \mathrm{R}, 0)$ for ranks 1, 5, 20. Each value is accompanied by an integer ranking across all algorithms. The shading indicates arguably the most important metric to operator-led media searching applications (e.g. broadcast news) where there is only mild intolerance for false positives. Note very high miss rates throughout, especially at low false positive rates. The FPIR(T) values are computed as the the number of false positives divided by the number of impostor videos used. This is an unconventional definition because the number of faces found in any given clip may exceed one, and each of these produces a template which is searched. This means FPIR here is an upper bound greater than or equal to its value if the number of faces present was known exactly.

Results V2S: The results are summarized in Table 31. It shows FNIR values aggregated over both trials by concatenating all scores and ranks. This step is defensible only because the two galleries have almost the same size, and are sampled randomly from the same parent image population.

The error rates are much higher than in other experiments documented in this report, reflecting the lack of geometric constraints on the photography, particularly in allowing and selecting highly variable head poses. Moreover, this factor applies to both the gallery and search imagery. All other tests in this report use standards-conformant frontal enrollments.

At high thresholds, FNIR is essentially $100 \%$ with NFP $=1$. Even allowing NFP $=100$, the lower threshold still yield a best FNIR(N, L, T) $=0.62$ from algorithm G32V. If such data was to be used with a human review, then the mate is not at rank one still quiet often: $\operatorname{FNIR}(\mathrm{N}, 1,0)=0.36(\mathrm{G} 32 \mathrm{~V})$ and not within the top 20 ranks at best with FNIR(N, 20, 0$)=0.064$ (M31V). This dataset is easier at rank 20, than is Dataset $\mathrm{H}$, despite the latter being easier at rank 1 i.e. the cumulative match characteristics cross.

\begin{tabular}{|l|l|l|l|}
\hline \multicolumn{4}{|c|}{ PARTICIPANT KEY } \\
\hline $\mathrm{A}=$ DIGITAL BARRIERS & $\mathrm{E}=$ NEUROTECHNOLOGY & $\mathrm{I}=$ EYEDEA & $\mathrm{M}=$ NEC \\
\hline $\mathrm{B}=$ HBINNO & $\mathrm{F}=$ VAPPLICA & $\mathrm{J}=$ HISIGN & $\mathrm{N}=$ TOSHIBA \\
\hline $\mathrm{C}=$ VIGILANT & $\mathrm{G}=$ MORPHO & $\mathrm{K}=$ COGNITEC & $\mathrm{Q}=$ IMAGUS \\
\hline $\mathrm{D}=$ AYONIX & $\mathrm{H}=$ 3M COGENT & $\mathrm{L}=$ CYBEREXTRUDER & $\mathrm{R}=$ RANK ONE \\
\hline
\end{tabular}

\begin{tabular}{|l|l|l|l|l|l|}
\hline SET & SCENE & CAMERA & SET & SCENE & CAMERA \\
\hline C & PHOTOJOURNALISM & PRO & T & CONCOURSE & PRO \\
\hline J & PASSENGER LOADING & PRO & H & CONCOURSE & PRO \\
\hline P & SPORTS ARENA & CONSUMER & U & CHOKEPOINT & WEBCAM \\
\hline L & LUGGAGE RACK & WEBCAM & & & \\
\hline
\end{tabular}


Results V2V: Figure 39 summarizes accuracy for the video-to-video case by showing $\operatorname{FNIR}(N, R, 0)$ i.e. miss rates at three ranks. We do not show high-threshold $\operatorname{FNIR}(N, L, T)$ as we did not run mateless video-to-video searches over which we could compute false positive outcomes. The algorithms given the best rank-1 miss rates are from providers $M$, G, I, H, N. Notably for rank-20 the K, E and B algorithms give low error rates, but $\mathrm{N}$ does not.

As with detection on prior datasets, the number of persons detected and enrolled into the gallery varies considerably. Algorithm E30V finds fewer people than are actually present. The most accurate algorithms, M3xV, find 2688 faces and A30V finds 11635. We do not have complete ground truth on the actual number of faces present, per the discussion in section 4.1 .

Some notable observations from Figure 39 are as follows. Some developers, N, M, J, I, H, appear use the same detection and tracking algorithms for all their submissions, producing identical numbers of tracks. However the feature extraction code sometimes differs. Thus, M31V produces accuracy better than M30V with enrollment templates three times smaller. N30V similarly has accuracy better than N32V but with a template four times smaller. I3xV doubles its template size between submissions, with the smaller template giving better accuracy.

Refering to the text annotations in Figure 39, over all algorithms, there is a massive variation in the amount of template data extracted from video: G30V extracts less than a $1 \mathrm{MB}$ from the entire video set, representing each of the 1078 detected faces with just 0.4 kilobytes. R30V is almost as parsimonious detecting slightly few faces (937) but encoding each with an average of 0.6 kilobytes. At the other end of the spectrum, the N33V enrollments average 1830 kilobytes, i.e. three orders of magnitude more per face than the R30V and G30V algorithms. The G31V, J3xV, and L30V algorithms are very economical also. Size may have implications for speed of searching large media collections.

\section{Computational resource requirements}

Face recognition engines detect and track faces in video clips, then produce searchable templates. While search duration is fast for small enrollment databases, the image processing and template generation stage is expensive. The time and memory resources it takes for an algorithm to generate a template and search it against a database determines hardware requirements. Furthermore, the size of templates produced can impact network bandwidth and disk space requirements.

\subsection{Test environment}

Software environment: The algorithms were submitted to NIST as pre-compiled libraries that implemented a NISTspecified C++ API [20]. The API declares the functions necessary to find and extract features from faces in still and video imagery. Source code is not provided to NIST: As such, this is a black box evaluation. NIST has no knowledge of how the implementations effect recognition.

Configuration data: Each algorithm was accompanied by configuration data.

Hardware: Testing was performed on high-end server-class blades, most of which were 6-core machines with dual processors running at $3.47 \mathrm{GHz}$ with $192 \mathrm{~GB}$ of main memory. GPUs were not used. IPP - Intel Integrated Performance Primitives was permitted if supporting libraries were delivered with submission.

Operating system: All processing was done on the CentOS 7.0 64-bit Linux variant. The test harness was built on top of the NIST Biometric Evaluation Framework [17] and used concurrent processing to distribute workload across dozens of

\begin{tabular}{|l|l|l|l|}
\hline \multicolumn{5}{|c|}{ PARTICIPANT KEY } \\
\hline $\mathrm{A}=$ DIGITAL BARRIERS & $\mathrm{E}=$ NEUROTECHNOLOGY & $\mathrm{I}=$ EYEDEA & $\mathrm{M}=$ NEC \\
\hline $\mathrm{B}=$ HBINNO & $\mathrm{F}=$ VAPPLICA & $\mathrm{J}=$ HISIGN & $\mathrm{N}=$ TOSHIBA \\
\hline $\mathrm{C}=$ VIGILANT & $\mathrm{G}=$ MORPHO & $\mathrm{K}=$ COGNITEC & $\mathrm{Q}=$ IMAGUS \\
\hline $\mathrm{D}=$ AYONIX & $\mathrm{H}=$ 3M COGENT & $\mathrm{L}=$ CYBEREXTRUDER & $\mathrm{R}=$ RANK ONE \\
\hline
\end{tabular}

\begin{tabular}{|l|l|l|l|l|l|}
\hline SET & SCENE & CAMERA & SET & SCENE & CAMERA \\
\hline C & PHOTOJOURNALISM & PRO & T & CONCOURSE & PRO \\
\hline J & PASSENGER LOADING & PRO & H & CONCOURSE & PRO \\
\hline P & SPORTS ARENA & CONSUMER & U & CHOKEPOINT & WEBCAM \\
\hline L & LUGGAGE RACK & WEBCAM & & & \\
\hline
\end{tabular}




\section{$\mathbf{R} 20 \bigcirc 5 \bigcirc 1$}

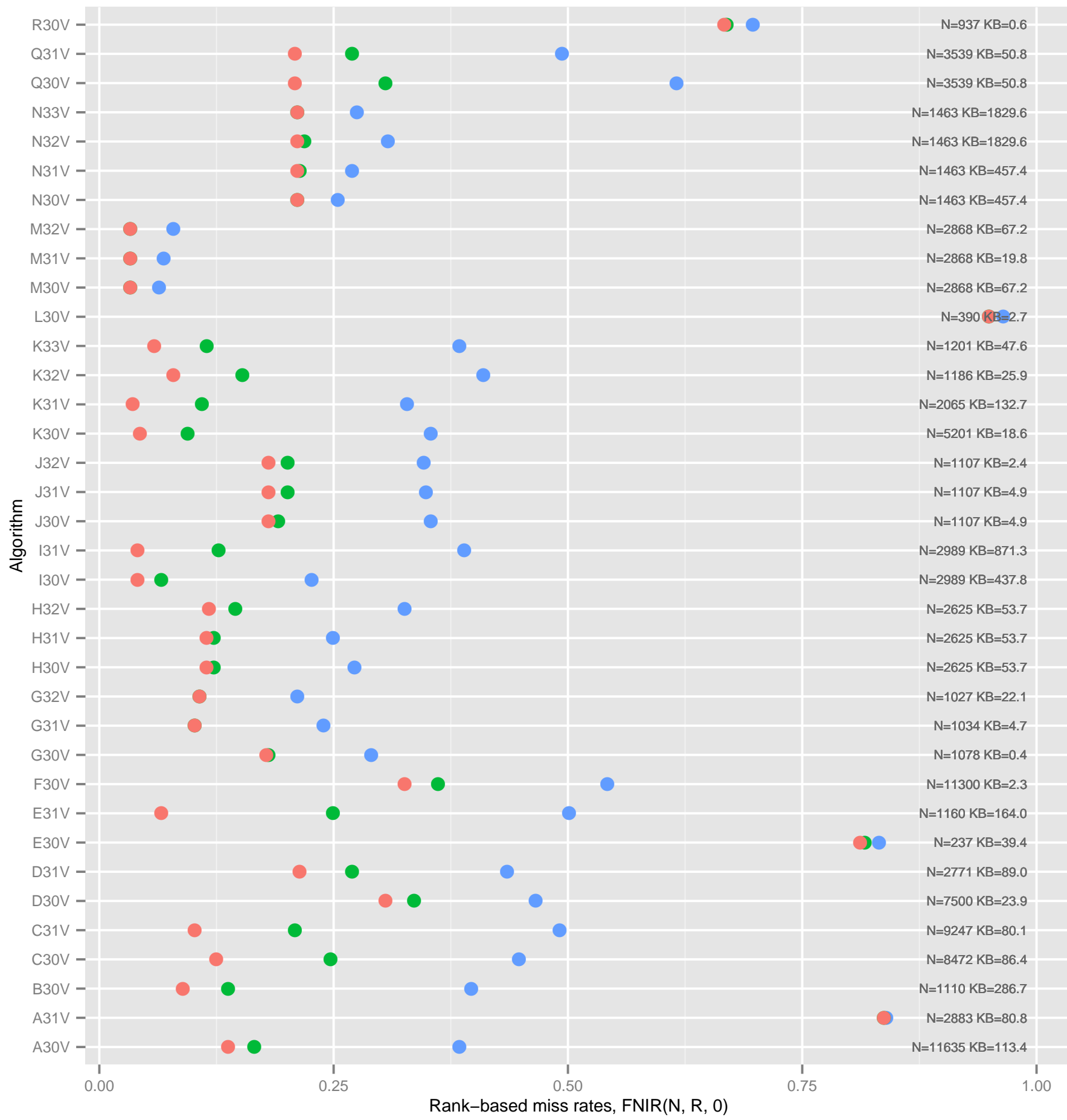

Figure 39: Video-to-video recognition: The dots show miss rates, $\operatorname{FNIR}(N, R, 0)$, for $R=\{1,5,20\}$ for each algorithm applied to DATASET C: PHOTOJOURNALISM imagery. The algorithms enroll variable numbers of templates from faces detected in the enrollment video clips. These numbers appear as text, and vary under the influence of false positive and false negative detection rates, tracking integrity rates, and image quality acceptance criteria. The template size is computed as the size of the finalized enrollment data divided by the number of persons detected. Note that the number of enrolled templates is generally higher than the 393 input videos because, in addition to the 393 known individuals, there are additionally an unknown number of other persons present. All of the 1356 search video clips contain at least one, and very rarely more, of the actors in the gallery. The probes also contain unknown faces.

\begin{tabular}{|c|c|c|c|c|c|c|c|c|c|}
\hline \multicolumn{4}{|c|}{ PARTICIPANT KEY } & \multirow{2}{*}{\begin{tabular}{|l|} 
SET \\
$\mathrm{C}$ \\
\end{tabular}} & SCENE & \multirow{2}{*}{\begin{tabular}{|l|} 
CAMERA \\
PRO
\end{tabular}} & \multirow{2}{*}{\begin{tabular}{|l|} 
SET \\
$T$
\end{tabular}} & \multirow{2}{*}{\begin{tabular}{|l|} 
SCENE \\
CONCOURSE \\
\end{tabular}} & \multirow{2}{*}{\begin{tabular}{|l|} 
CAMERA \\
PRO
\end{tabular}} \\
\hline $\mathrm{A}=$ DIGITAL $\mathrm{B}$. & $E=$ NEUROTECHNOLOGY & $I=$ EYEDEA & $M=N E C$ & & PHOTOJOURNALISM & & & & \\
\hline $\mathrm{C}=$ VIGILANT & $G=\mathrm{MORPHO}$ & $\mathrm{K}=$ COGNITEC & $Q=$ IMAGUS & $P$ & SPORTS ARENA & CONSUMER & $\mathrm{U}$ & CHOKEPOINT & WEBCAM \\
\hline $\mathrm{D}=\mathrm{AYONIX}$ & $\mathrm{H}=3 \mathrm{M}$ COGENT & $\mathrm{L}=$ CYBEREXTRUDER & $\mathrm{R}=\mathrm{RANK}$ ONE & $\mathrm{L}$ & LUGGAGE RACK & WEBCAM & & & \\
\hline
\end{tabular}




\begin{tabular}{|c|c|c|c|c|c|c|c|}
\hline \multicolumn{2}{|c|}{ Algorithm } & \multicolumn{2}{|c|}{ Algorithm } & \multicolumn{2}{|c|}{ Algorithm } & \multicolumn{2}{|c|}{ Algorithm } \\
\hline A30V & 104 & A31V & 104 & & & & \\
\hline B30V & 1 & & & & & & \\
\hline C30V & 173 & C31V & 173 & & & & \\
\hline D30V & 95 & D31V & 95 & & & & \\
\hline E30V & 99 & E31V & 99 & & & & \\
\hline F30V & 122 & & & & & & \\
\hline G30V & 1646 & G31V & 1647 & G32V & 1647 & & \\
\hline H30V & 155 & H31V & 155 & $\mathrm{H} 32 \mathrm{~V}$ & 155 & & \\
\hline I30V & 746 & I31V & 745 & & & & \\
\hline $\mathrm{J} 30 \mathrm{~V}$ & 388 & J31V & 433 & $\mathrm{~J} 32 \mathrm{~V}$ & 249 & & \\
\hline K30V & 684 & K31V & 684 & K32V & 684 & K33V & 684 \\
\hline L30V & 604 & & & & & & \\
\hline M30V & 284 & M31V & 284 & M32V & 284 & & \\
\hline N30V & 317 & N31V & 317 & N32V & 317 & N33V & 317 \\
\hline Q30V & 47 & Q31V & 47 & & & & \\
\hline R30V & 119 & & & & & & \\
\hline
\end{tabular}

Table 32: For each algorithm, sizes in megabytes, of static read-only configuration data supplied with the algorithm, as reported by the unix command "du -sm".

blades.

\subsection{Configuration directory size}

Participants were permitted to provide static, read-only configuration data with their algorithm submission. The location of such configuration data is provided to the implementation during algorithm initialization for template generation and search. Configuration data typically includes trained feature extraction models, but here, the content is entirely unregulated. Table 32 reports the size of the configuration data for each algorithm.

\subsection{Video processing time}

Face recognition in video sequences implies computational cost over recognition using still images. A portrait photograph can be enrolled in less than a second [21]. In video however, two factors imply slower processing. First, video imagery typically has larger width and height, as cameras often have wider fields of view. Second, there are many frames, and processing times scale, to first order, linearly with the frame rate. Given 24 or 30 frames per second the costs are considerable. This section details cost.

Video processing time refers to the amount of time that elapsed while a video sequence was processed by a recognition algorithm. The primary purpose of processing a video is to produce matchable templates. Reported times do not include any pre-processing steps performed by the testing harness such as loading the video from disk. The timing machine ran with an Intel Xeon E5-2695 v3 @ 2.30 GHz, 56 logical CPUs, 528 GB RAM.

Generating a template from a video sequence is computationally intensive and generally scales with the number of frames in the video sequence but depends on other factors as well. For example, Table 33 demonstrates that all algorithms require greater processing time as the number of people in the videos increases. This component of the duration includes the time taken for any fine localization and alignment, and feature extraction.

\begin{tabular}{|l|l|l|l|}
\hline \multicolumn{5}{|c|}{ PARTICIPANT KEY } \\
\hline $\mathrm{A}=$ DIGITAL BARRIERS & E = NEUROTECHNOLOGY & I = EYEDEA & $\mathrm{M}=$ NEC \\
\hline $\mathrm{B}=$ HBINNO & $\mathrm{F}=$ VAPPLICA & $\mathrm{J}=$ HISIGN & $\mathrm{N}=$ TOSHIBA \\
\hline $\mathrm{C}=$ VIGILANT & $\mathrm{G}=$ MORPHO & $\mathrm{K}=$ COGNITEC & $\mathrm{Q}=$ IMAGUS \\
\hline $\mathrm{D}=$ AYONIX & $\mathrm{H}=3 \mathrm{M}$ COGENT & $\mathrm{L}=$ CYBEREXTRUDER & $\mathrm{R}=$ RANK ONE \\
\hline
\end{tabular}

\begin{tabular}{|l|l|l|l|l|l|}
\hline SET & SCENE & CAMERA & SET & SCENE & CAMERA \\
\hline C & PHOTOJOURNALISM & PRO & T & CONCOURSE & PRO \\
\hline J & PASSENGER LOADING & PRO & H & CONCOURSE & PRO \\
\hline P & SPORTS ARENA & CONSUMER & U & CHOKEPOINT & WEBCAM \\
\hline L & LUGGAGE RACK & WEBCAM & & & \\
\hline
\end{tabular}




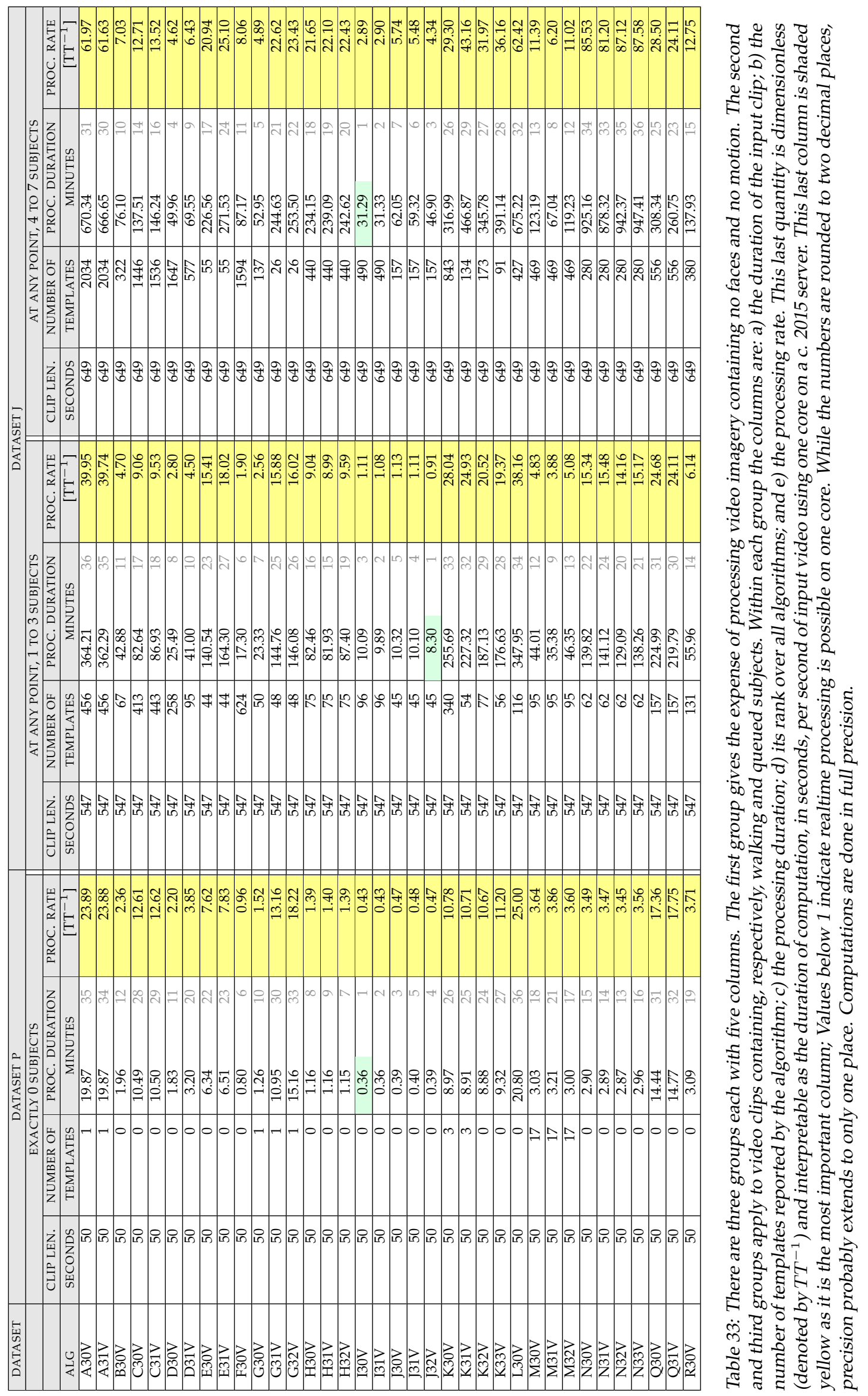

\begin{tabular}{|l|l|l|l|}
\hline \multicolumn{4}{|c|}{ PARTICIPANT KEY } \\
\hline $\mathrm{A}=$ DIGITAL BARRIERS & E = NEUROTECHNOLOGY & I = EYEDEA & $\mathrm{M}=$ NEC \\
\hline $\mathrm{B}=$ HBINNO & $\mathrm{F}=$ VAPPLICA & $\mathrm{J}=$ HISIGN & $\mathrm{N}=$ TOSHIBA \\
\hline $\mathrm{C}=$ VIGILANT & $\mathrm{G}=$ MORPHO & $\mathrm{K}=$ COGNITEC & $\mathrm{Q}=$ IMAGUS \\
\hline $\mathrm{D}=$ AYONIX & $\mathrm{H}=$ 3M COGENT & $\mathrm{L}=$ CYBEREXTRUDER & $\mathrm{R}=$ RANK ONE \\
\hline
\end{tabular}

\begin{tabular}{|l|l|l|l|l|l|}
\hline SET & SCENE & CAMERA & SET & SCENE & CAMERA \\
\hline C & PHOTOJOURNALISM & PRO & T & CONCOURSE & PRO \\
\hline J & PASSENGER LOADING & PRO & H & CONCOURSE & PRO \\
\hline P & SPORTS ARENA & CONSUMER & U & CHOKEPOINT & WEBCAM \\
\hline L & LUGGAGE RACK & WEBCAM & & & \\
\hline
\end{tabular}




\begin{tabular}{|c|c|c|c|c|}
\hline Alg & Template Size (bytes) & Size Rank & Template Generation Time (sec) & Time Rank \\
\hline A30V & 5070 & 22 & $0.81 \quad(\sigma=0.12)$ & 30 \\
\hline A31V & 5070 & 22 & $0.87(\sigma=0.16)$ & 31 \\
\hline B30V & 1060 & 4 & $0.08 \quad(\sigma=0.02)$ & 2 \\
\hline C30V & $109150 \quad(\sigma=33810)$ & 36 & $1.32(\sigma=0.35)$ & 35 \\
\hline $\mathrm{C} 31 \mathrm{~V}$ & $109150 \quad(\sigma=33810)$ & 36 & $1.29(\sigma=0.34)$ & 34 \\
\hline D30V & 1140 & 6 & $0.06 \quad(\sigma=0.01)$ & 1 \\
\hline D31V & 1140 & 6 & $0.09 \quad(\sigma=0.03)$ & 3 \\
\hline E30V & 10251 & 29 & $0.64(\sigma=0.12)$ & 23 \\
\hline E31V & 42219 & 32 & $0.79(\sigma=0.17)$ & 29 \\
\hline F30V & $2268(\sigma=111)$ & 10 & $0.12(\sigma=0.01)$ & 5 \\
\hline G30V & $459 \quad(\sigma=20)$ & 2 & $0.19(\sigma=0.01)$ & 8 \\
\hline G31V & $4990(\sigma=227)$ & 18 & $1.49(\sigma=0.08)$ & 36 \\
\hline G32V & $5736(\sigma=261)$ & 27 & $1.22(\sigma=0.06)$ & 32 \\
\hline H30V & 2021 & 8 & $0.67 \quad(\sigma=0.04)$ & 24 \\
\hline $\mathrm{H} 31 \mathrm{~V}$ & 2021 & 8 & $0.71 \quad(\sigma=0.08)$ & 28 \\
\hline $\mathrm{H} 32 \mathrm{~V}$ & 2021 & 8 & $0.68 \quad(\sigma=0.06)$ & 25 \\
\hline $\mathrm{I} 30 \mathrm{~V}$ & $4098(\sigma=375)$ & 17 & $0.68(\sigma=0.09)$ & 26 \\
\hline $\mathrm{I} 31 \mathrm{~V}$ & $8152(\sigma=747)$ & 28 & $0.68(\sigma=0.07)$ & 27 \\
\hline J30V & 5048 & 20 & $0.15(\sigma=0.03)$ & 6 \\
\hline $\mathrm{J} 31 \mathrm{~V}$ & 5048 & 20 & $0.15(\sigma=0.02)$ & 7 \\
\hline $\mathrm{J} 32 \mathrm{~V}$ & 2532 & 11 & $0.12(\sigma=0.02)$ & 4 \\
\hline K30V & 5368 & 24 & $0.30 \quad(\sigma=0.05)$ & 10 \\
\hline K31V & 5368 & 24 & $0.30(\sigma=0.04)$ & 11 \\
\hline K32V & 5368 & 24 & $0.30(\sigma=0.05)$ & 13 \\
\hline K33V & 5368 & 24 & $0.30(\sigma=0.05)$ & 12 \\
\hline L30V & 1032 & 3 & $1.26(\sigma=0.15)$ & 33 \\
\hline M30V & 2585 & 13 & $0.34(\sigma=0.03)$ & 16 \\
\hline M31V & 2585 & 13 & $0.32(\sigma=0.02)$ & 15 \\
\hline M32V & 2585 & 13 & $0.32(\sigma=0.01)$ & 14 \\
\hline N30V & 3134 & 16 & $0.58 \quad(\sigma=0.06)$ & 22 \\
\hline N31V & 3134 & 16 & $0.58 \quad(\sigma=0.05)$ & 21 \\
\hline N32V & 12350 & 30 & $0.57(\sigma=0.05)$ & 20 \\
\hline N33V & 12350 & 30 & $0.57 \quad(\sigma=0.05)$ & 19 \\
\hline Q30V & 53264 & 34 & $0.46 \quad(\sigma=0.17)$ & 18 \\
\hline Q31V & 53264 & 34 & $0.41 \quad(\sigma=0.15)$ & 17 \\
\hline R30V & 128 & 1 & $0.21 \quad(\sigma=0.09)$ & 9 \\
\hline
\end{tabular}

Table 34: Mean template size and generation time for each submission over 480 still face images from DATASET J: PASSENGER LOADING BRIDGE . Standard deviations in parenthesis when there is variation. The mean interocular distance is 118 pixels.

\subsection{Still face template size}

The time it takes to generate templates for still face images is less operationally relevant since it is only a factor when the database is being generated or altered. Its computation time is tiny compared to the time it takes to generate templates for video face tracks (see Table 34). The size of the templates will affect disk space requirements. For templates generated from a single still-face image, sizes range from 128 bytes (R30V) to 109,150 bytes (C30V, and C31V). Many systems operate by loading the entire database into memory to expedite matching. In this case, a system would need $109 \mathrm{MB}$ of memory for a database of size 1,000 and 10.3 GB for a database of size 100,000.

Back in section 5.5.2 the effect of enrolling $K=3$ images per subject was examined. Table 35 demonstrates that in such cases template generation time usually scales about linearly with the number of input faces. This is true in most cases, but with the exceptions of F30V, G30V, G31V, J30V, J31V, and J32V which maintain approximately constant size. This may occur because only one image is used, for example the "best" one, or because information is being fused across all $K$ inputs, either at the image level (modeling) or at the feature level.

\begin{tabular}{|l|l|l|l|}
\hline \multicolumn{5}{|c|}{ PARTICIPANT KEY } \\
\hline $\mathrm{A}=$ DIGITAL BARRIERS & $\mathrm{E}=$ NEUROTECHNOLOGY & $\mathrm{I}=$ EYEDEA & $\mathrm{M}=$ NEC \\
\hline $\mathrm{B}=$ HBINNO & $\mathrm{F}=$ VAPPLICA & $\mathrm{J}=$ HISIGN & $\mathrm{N}=$ TOSHIBA \\
\hline $\mathrm{C}=$ VIGILANT & $\mathrm{G}=$ MORPHO & $\mathrm{K}=$ COGNITEC & $\mathrm{Q}=$ IMAGUS \\
\hline $\mathrm{D}=$ AYONIX & $\mathrm{H}=$ 3M COGENT & $\mathrm{L}=$ CYBEREXTRUDER & $\mathrm{R}=$ RANK ONE \\
\hline
\end{tabular}

\begin{tabular}{|l|l|l|l|l|l|}
\hline SET & SCENE & CAMERA & SET & SCENE & CAMERA \\
\hline C & PHOTOJOURNALISM & PRO & T & CONCOURSE & PRO \\
\hline J & PASSENGER LOADING & PRO & H & CONCOURSE & PRO \\
\hline P & SPORTS ARENA & CONSUMER & U & CHOKEPOINT & WEBCAM \\
\hline L & LUGGAGE RACK & WEBCAM & & & \\
\hline
\end{tabular}


Table 35: Mean template sizes in bytes, and mean template generation times in milliseconds, for each submission over 56 still face images from the DATASET H: TRAVEL WALKWAY dataset. The column heading numbers give the number of still images passed to the template generation function. The numbers in light grey are the factor increase compared to the 1-pose column. The mean interocular distance is 199 pixels. The timing numbers are sometimes slower than the formal estimates over the larger set given in Table 34 due to higher resolution.

\begin{tabular}{|c|c|c|c|c|c|c|c|c|c|c|c|c|c|c|}
\hline Alg & Size1 & Size3 & & Size5 & & Size7 & & Time1 & Time3 & & Time5 & & Time? & \\
\hline A30V & 5070 & 14857 & 2.9 & 24553 & 4.8 & 33016 & 6.5 & 1340 & 3924 & 2.9 & 6551 & 4.9 & 9106 & 6.8 \\
\hline A31V & 5070 & 14857 & 2.9 & 24553 & 4.8 & 33016 & 6.5 & 1371 & 4006 & 2.9 & 6516 & 4.8 & 8904 & 6.5 \\
\hline B30V & 1060 & 3148 & 3.0 & 5236 & 4.9 & 7324 & 6.9 & 55 & 165 & 3.0 & 267 & 4.9 & 378 & 6.9 \\
\hline C30V & 120593 & 373575 & 3.1 & 621151 & 5.2 & 866837 & 7.2 & 1263 & 3952 & 3.1 & 6528 & 5.2 & 9021 & 7.1 \\
\hline C31V & 120593 & 373575 & 3.1 & 621151 & 5.2 & 866837 & 7.2 & 1292 & 3888 & 3.0 & 6542 & 5.1 & 9592 & 7.4 \\
\hline D30V & 21140 & 3388 & 3.0 & 5616 & 4.9 & 7802 & 6.8 & 103 & 301 & 2.9 & 4966 & 44.8 & 692 & 6.7 \\
\hline D31V & 1140 & 3388 & 3.0 & 5616 & 4.9 & 7782 & 6.8 & 237 & 678 & 2.9 & 866 & 3.7 & 1163 & 4.9 \\
\hline E30V & 10251 & 30713 & 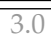 & 51175 & 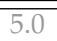 & 71637 & 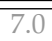 & 660 & 1791 & 2.7 & 4080 & 6.2 & 4191 & $\bar{~} 6.3$ \\
\hline E31V & 42219 & 126617 & 3.0 & 211015 & 5.0 & 295413 & 7.0 & 783 & 2189 & 2.8 & 3764 & 4.8 & 5151 & 6.6 \\
\hline F30V & 2267 & 2308 & 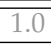 & 2896 & 1.3 & 3142 & 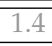 & 1111 & 314 & 2.8 & 503 & 4.5 & $\overline{c 656}$ & 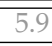 \\
\hline G30V & 460 & 436 & 0.9 & 436 & 0.9 & 436 & 0.9 & 206 & 596 & 2.9 & 1000 & 4.9 & 1484 & $\overline{77.2}$ \\
\hline G31V & 5000 & 4976 & 1.0 & 4976 & 1.0 & 4976 & 1.0 & 1400 & 4316 & 3.1 & 7347 & 5.2 & 9887 & 7.1 \\
\hline G32V & 5748 & 17220 & 3.0 & 28692 & 5.0 & 40062 & 7.0 & 1236 & 3682 & 3.0 & 6160 & 5.0 & 8938 & 7.2 \\
\hline H30V & 2021 & 5991 & 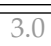 & $\overline{99925}$ & 4.9 & 13858 & 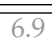 & 707 & 21928 & 2.7 & 3334 & 4.7 & 24541 & $\overline{76.4}$ \\
\hline H31V & 2021 & 5991 & 3.0 & 9925 & 4.9 & 13858 & 6.9 & 640 & 1925 & 3.0 & 3231 & 5.0 & 4556 & 7.1 \\
\hline $\mathrm{H} 32 \mathrm{~V}$ & 2021 & 5991 & 3.0 & 9925 & 4.9 & 13858 & 6.9 & 782 & 1908 & 2.4 & 3328 & 4.3 & 4562 & 5.8 \\
\hline$\overline{\mathrm{I} \text { I30V }}$ & 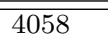 & 12241 & 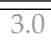 & 20128 & 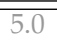 & 27280 & 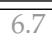 & $\overline{2633}$ & 1947 & 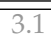 & 3206 & 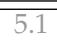 & 4365 & $\overline{76.9}$ \\
\hline $\mathrm{I} 31 \mathrm{~V}$ & 8073 & 24359 & 3.0 & 40058 & 5.0 & 54290 & 6.7 & 770 & 1987 & 2.6 & 3214 & 4.2 & 4409 & 5.7 \\
\hline J30V & 5048 & 5048 & 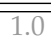 & 5048 & $\begin{array}{l}1.0 \\
\end{array}$ & 5048 & 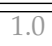 & 208 & 526 & 2.5 & 842 & 4.0 & 1163 & $\overline{5.6}$ \\
\hline $\mathrm{J} 31 \mathrm{~V}$ & 5048 & 5048 & 1.0 & 5048 & 1.0 & 5048 & 1.0 & 222 & 540 & 2.4 & 860 & 3.9 & 1265 & 5.7 \\
\hline $\mathrm{J} 32 \mathrm{~V}$ & 2532 & 2532 & 1.0 & 2532 & 1.0 & 2532 & 1.0 & 185 & 501 & 2.7 & 819 & 4.4 & 1161 & 6.3 \\
\hline K30V & 25368 & 15809 & 2.9 & 26154 & 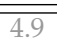 & 26345 & 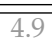 & 600 & 1766 & 2.9 & 2927 & 74.9 & 24040 & $\overline{c 6.7}$ \\
\hline K31V & 5368 & 16000 & 3.0 & 26537 & 4.9 & 37073 & 6.9 & 581 & 1769 & 3.0 & 2931 & 5.0 & 4076 & 7.0 \\
\hline K32V & 5368 & 15809 & 2.9 & 26154 & 4.9 & 26345 & 4.9 & 578 & 1761 & 3.0 & 2921 & 5.1 & 4057 & 7.0 \\
\hline K33V & 5368 & 16000 & 3.0 & 26537 & 4.9 & 37073 & 6.9 & 578 & 1765 & 3.1 & 2948 & 5.1 & 4078 & 7.1 \\
\hline L30V & 1032 & $\begin{array}{c}4104 \\
\end{array}$ & 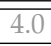 & 6115 & 5.9 & 6152 & 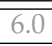 & 1744 & 46647 & 2.7 & 8291 & 4.8 & 9865 & 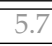 \\
\hline M30V & 2585 & 7755 & 3.0 & 12740 & 4.9 & 17818 & $\overline{26.9}$ & 371 & 1082 & 2.9 & 1800 & 4.9 & 2526 & $\overline{26.8}$ \\
\hline M31V & 2585 & 7755 & 3.0 & 12740 & 4.9 & 17818 & 6.9 & 362 & 1096 & 3.0 & 1786 & 4.9 & 2515 & 6.9 \\
\hline M32V & 2585 & 7755 & 3.0 & 12740 & 4.9 & 17818 & 6.9 & 371 & 1088 & 2.9 & 1804 & 4.9 & 2528 & 6.8 \\
\hline N30V & 3134 & 9278 & 3.0 & 15422 & 4.9 & 21566 & $\overline{26.9}$ & 2657 & 21985 & 3.0 & 3680 & 5.6 & 5385 & $\overline{8.2}$ \\
\hline N31V & 3134 & 9278 & 3.0 & 15422 & 4.9 & 21566 & 6.9 & 659 & 2011 & 3.1 & 3727 & 5.7 & 5377 & 8.2 \\
\hline N32V & 12350 & 36926 & 3.0 & 61502 & 5.0 & 86078 & 7.0 & 656 & 2008 & 3.1 & 3662 & 5.6 & 5299 & 8.1 \\
\hline N33V & 12350 & 36926 & 3.0 & 61502 & 5.0 & 86078 & 7.0 & 659 & 2020 & 3.1 & 3666 & 5.6 & 5298 & 8.0 \\
\hline Q30V & 53264 & 146476 & 2.8 & 245395 & 4.6 & 316731 & $\bar{~} 5.9$ & $\overline{21091}$ & 3254 & 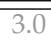 & 5435 & $\overline{5.0}$ & 7602 & $\overline{77.0}$ \\
\hline Q31V & 53264 & 146476 & 2.8 & 245395 & 4.6 & 316731 & 5.9 & 1040 & 3179 & 3.1 & 5215 & 5.0 & 7294 & 7.0 \\
\hline R30V & 128 & 144 & 1.1 & 190 & 1.5 & 208 & 1.6 & 340 & 942 & 2.8 & 1531 & 4.5 & 2100 & 6.2 \\
\hline
\end{tabular}

\subsection{Memory usage during video processing}

The FIVE approach to video processing is to pass an entire video clip to the algorithm via a single function call invocation. The size of the input data is equal to the product of the width of the image, the height, the number of color channels (always 3 here) and the number of frames. The largest single video clips in this study were those from the simulated aircraft boarding of DATASET J: PASSENGER LOADING BRIDGE, extending to about 12 minutes in length. Given 1920x1080 frames, 30 frames per second, the uncompressed data requires about 125 gigabytes of storage. Total memory requirements exceed that because algorithms generally allocate working memory. This is recorded for two somewhat shorter video clips in Figure 40. The plot shows, as vertical lines, the baseline amount of memory used by a dummy NIST implementation which did no computation.

\begin{tabular}{|l|l|l|l|}
\hline \multicolumn{4}{|c|}{ PARTICIPANT KEY } \\
\hline $\mathrm{A}=$ DIGITAL BARRIERS & E = NEUROTECHNOLOGY & $\mathrm{I}=$ EYEDEA & $\mathrm{M}=$ NEC \\
\hline $\mathrm{B}=$ HBINNO & $\mathrm{F}=$ VAPPLICA & $\mathrm{J}=$ HISIGN & $\mathrm{N}=$ TOSHIBA \\
\hline $\mathrm{C}=$ VIGILANT & $\mathrm{G}=$ MORPHO & $\mathrm{K}=$ COGNITEC & $\mathrm{Q}=$ IMAGUS \\
\hline $\mathrm{D}=$ AYONIX & $\mathrm{H}=$ 3M COGENT & $\mathrm{L}=$ CYBEREXTRUDER & $\mathrm{R}=$ RANK ONE \\
\hline
\end{tabular}

\begin{tabular}{|l|l|l|l|l|l|}
\hline SET & SCENE & CAMERA & SET & SCENE & CAMERA \\
\hline C & PHOTOJOURNALISM & PRO & T & CONCOURSE & PRO \\
\hline J & PASSENGER LOADING & PRO & H & CONCOURSE & PRO \\
\hline P & SPORTS ARENA & CONSUMER & U & CHOKEPOINT & WEBCAM \\
\hline L & LUGGAGE RACK & WEBCAM & & & \\
\hline
\end{tabular}


The algorithms generally consume very litle additional memory. The exceptions are the algorithms from the J, K, L and I developers. The $\mathrm{K}$ algorithms use more than double the input data size consistent with making a copy of the input data. We assume this is unnecessary and therefore not material to algorithm selection.

The FIVE measurements of memory use are likely representative to applications which do offline processing of video. For cases where video is streamed continuously to a face recognition system, programmers will need to architect a solution that operates on a first-in first-out buffer that, to first order, is sized about the length of the subject appearance, and is long enough to afford good tracking, noise suppression, and feature extraction.

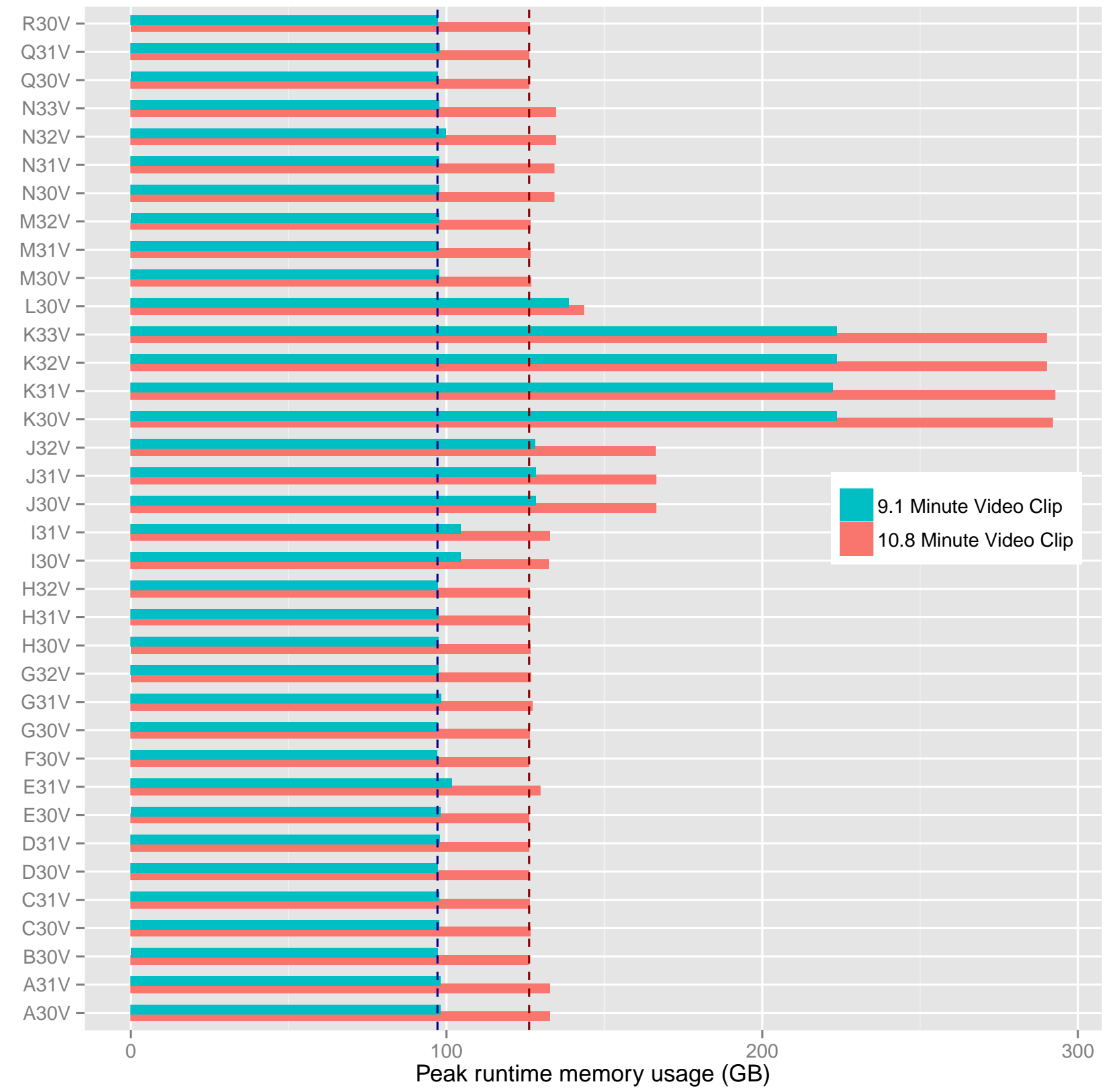

Figure 40: Maximum memory usage during video processing (template generation) using shorter and longer video clips from DATASET J: PASSENGER LOADING BRIDGE. The dotted lines mark how much memory was required to load the raw video data (independent of the matching software).

\begin{tabular}{|l|l|l|l|}
\hline \multicolumn{4}{|c|}{ PARTICIPANT KEY } \\
\hline $\mathrm{A}=$ DIGITAL BARRIERS & E = NEUROTECHNOLOGY & $\mathrm{I}=$ EYEDEA & $\mathrm{M}=$ NEC \\
\hline $\mathrm{B}=$ HBINNO & F = VAPPLICA & $\mathrm{J}=$ HISIGN & $\mathrm{N}=$ TOSHIBA \\
\hline $\mathrm{C}=$ VIGILANT & $\mathrm{G}=$ MORPHO & K = COGNITEC & $\mathrm{Q}=$ IMAGUS \\
\hline $\mathrm{D}=$ AYONIX & $\mathrm{H}=$ 3M COGENT & $\mathrm{L}=$ CYBEREXTRUDER & $\mathrm{R}=$ RANK ONE \\
\hline
\end{tabular}

\begin{tabular}{|l|l|l|l|l|l|}
\hline SET & SCENE & CAMERA & SET & SCENE & CAMERA \\
\hline C & PHOTOJOURNALISM & PRO & T & CONCOURSE & PRO \\
\hline $\mathrm{J}$ & PASSENGER LOADING & PRO & H & CONCOURSE & PRO \\
\hline P & SPORTS ARENA & CONSUMER & U & CHOKEPOINT & WEBCAM \\
\hline L & LUGGAGE RACK & WEBCAM & & & \\
\hline
\end{tabular}




\section{References}

[1] United states of america vs. dow chemical. Technical Report 84-1259. 476 U.S. 227, Supreme Court of the United States, May 1986. https://www.law.uh.edu/faculty/thester/courses/Emerging

[2] Kyllo v. united states. Technical Report 99-8508. 533 U.S. 27, Supreme Court of the United States, June 2001. https://supreme.justia.com/cases/federal/us/533/27/.

[3] Face recognition as a search tool foto-fahndung. Technical report, Bundeskriminamt (BKA), Thaerstrasse 11, 65193, Wiesbaden, Germany, February 2007.

[4] Striking the balance - a government approach to facial recognition privacy and civil liberties. Technical report, Federal Bureau of Investigation, FBI National Academy, Quantico, Virginia, March 2012. https:/ / www.fbi.gov / filerepository/about-us-cjis-fingerprints_biometrics-biometric-center-of-excellences-forum_3_minutes.pdf.

[5] United states of america vs. leonel michel vargas. Technical Report 13-6025-EFS, United States District Court Eastern District of Washington, December 2014. https:/ / www.eff.org/files/2014/12/15/vargas_order.pdf.

[6] A national surveillance camera strategy for england and wales [draft]. Technical report, Surveillance Camera Commissioner, First Floor, Peel Building, 2 Marsham Street, London, SW1P 4DF, October 2016. https://www.gov.uk/government/uploads/system/uploads/attachment_data/file/561520/NSCS_Strategy_FINAL.pdf.

[7] United states of america vs. rocky joe houston. Technical Report 14-5800, United States Court of Appeals for the Sixth Circuit, February 2016. http://www.opn.ca6.uscourts.gov/opinions.pdf/16a0031p-06.pdf.

[8] V. Biaud, C. Herold, V. Despiegel, and S. Gentric. Semi-supervised evaluation of face recognition in videos. In Proc. Third International Biometrics Performance Conference, Gaithersburg (MD), 42014. https://www.nist.gov/itl/iad/image-group/ibpc-2014-presentations.

[9] Marc Jonathan Blitz. The fourth amendment future of public surveillance: Remote recording and other searches in public space. American University Law Review, 63(Issue 1 Article 2), 2013.

[10] Blumstein, Cohen, Roth, and Visher, editors. Random parameter stochastic models of criminal careers. National Academy of Sciences Press, 1986.

[11] Jeremy Brown. Pan, tilt, zoom: Regulating the use of video surveillance of public places. Berkeley Technology Law Journal, 23(Issue 1 Article 33), January 2008. http://scholarship.law.berkeley.edu/btlj/vol23/iss1/33.

[12] Mark Burge. Janus program broad agency announcement. $\quad$ Technical Report IARPA-BAA-13-07, Intelligence Advanced Research Projects Activity, $12 \quad 2013$. https:/ / www.fbo.gov/index?s=opportunity\&id=9af4574d0fcf89a2fbcd9eb2d8c8921f.

[13] Jordan Cheney, Ben Klein, Anil K. Jain, and Brendan F. Klare. Unconstrained face detection: State of the art baseline and challenges. In Proc. Eighth IAPR International Conference on Biometrics (ICB), May 2015.

[14] White D., Kemp R. I., Jenkins R., Matheson M, and Burton A. M. Passport officers errors in face matching. PLoS ONE, 9(8), 2014. e103510. doi:10.1371/journal. pone.0103510.

\begin{tabular}{|l|l|l|l|}
\hline \multicolumn{5}{|c|}{ PARTICIPANT KEY } \\
\hline A = DIGITAL BARRIERS & E = NEUROTECHNOLOGY & I = EYEDEA & $\mathrm{M}=$ NEC \\
\hline B = HBINNO & F = VAPPLICA & J = HISIGN & N = TOSHIBA \\
\hline C = VIGILANT & G = MORPHO & K = COGNITEC & Q = IMAGUS \\
\hline D = AYONIX & $\mathrm{H}=$ MM COGENT & L = CYBEREXTRUDER & R = RANK ONE \\
\hline
\end{tabular}

\begin{tabular}{|l|l|l|l|l|l|}
\hline SET & SCENE & CAMERA & SET & SCENE & CAMERA \\
\hline C & PHOTOJOURNALISM & PRO & T & CONCOURSE & PRO \\
\hline J & PASSENGER LOADING & PRO & H & CONCOURSE & PRO \\
\hline P & SPORTS ARENA & CONSUMER & U & CHOKEPOINT & WEBCAM \\
\hline L & LUGGAGE RACK & WEBCAM & & & \\
\hline
\end{tabular}


[15] Working Group 3. Ed. D. D'Amato. ISO/IEC 19794-5 Amendment 1 - Biometric data interchange formats - Part 5: Face image data - Conditions for taking photographs for face image data. JTC1 :: SC37, 1 edition, 122007. http://webstore.ansi.org.

[16] Stephance Gentric et al. Border control: From technical to operational evaluation. In Proceedings of the Second International Biometrics Performance Conference, NIST, Gaithersburg, MD, March 2012.

[17] Gregory Fiumara, Wayne Salamon, and Craig Watson. Towards Repeatable, Reproducible, and Efficient Biometric Technology Evaluations. In Biometrics Theory, Applications and Systems (BTAS), 2015 IEEE 7th International Conference on, pages 1-8, Sept 2015.

[18] Working Group 3. Ed. P. Griffin. ISO/IEC 19794-5 Information Technology - Biometric Data Interchange Formats - Part 5: Face image data. JTC1 :: SC37, 1 edition, 2005. http://webstore.ansi.org.

[19] P. Grother, G. W. Quinn, and P. J. Phillips. Evaluation of 2d still-image face recognition algorithms. NIST Interagency Report 7709, National Institute of Standards and Technology, 8 2010. http:/ / face.nist.gov/mbe as MBE2010 FRVT2010.

[20] Patrick Grother and Mei Ngan. Face In Video Evaluation (FIVE) Concept, Evaluation Plan, and API, November 2014. http://biometrics.nist.gov/cS_links/face/five/NIST_FIVE_API_2014Nov19.pdf.

[21] Patrick Grother and Mei Ngan. Interagency report 8009, performance of face identification algorithms. Face Recognition Vendor Test (FRVT), May 2014.

[22] Patrick Grother and Jonathon Phillips. Models of large population recognition performance. In Proc. IEEE Conference on Computer Vision and Pattern Recognition (CVPR), Washington, DC, volume 2, pages 68-75, June 2004.

[23] Patrick Grother, George W. Quinn, and Mei Ngan. Face recognition vendor test - still face image and video concept, evaluation plan and api. Technical report, National Institute of Standards and Technology, 72013. http://biometrics.nist.gov/cs_links/face/frvt/frvt2012/NIST_FRVT2012_api_Aug15.pdf.

[24] Gary B. Huang and Erik Learned-Miller. Labeled faces in the wild: Updates and new reporting procedures. Technical Report Technical Report UM-CS-2014-003, University of Massachusetts, Amherst, 42014.

[25] Ira Kemelmacher-Shlizerman, Steven M. Seitz, Daniel Miller, and Evan Brossard. The megaface benchmark: 1 million faces for recognition at scale. CoRR, abs/1512.00596, 2015.

[26] Brendan F. Klare, Ben Klein, Emma Taborsky, Austin Blanton, Jordan Cheney, Kristen Allen, Patrick Grother, Alan Mah, Mark J. Burge, and Anil K. Jain. Pushing the frontiers of unconstrained face detection and recognition: IARPA janus benchmark A. In IEEE Conference on Computer Vision and Pattern Recognition, CVPR 2015, Boston, MA, USA, June 7-12, 2015, pages 1931-1939, 2015. http:/ /dx.doi.org/10.1109/CVPR.2015.7298803.

[27] Yann LeCun, Yoshua Bengio, and Geoffrey Hinton. Deep learning. Nature, 521:436 - 444, 52015.

[28] Kuang-Chih Lee, Jeffrey Ho, Ming-Hsuan Yang, and David J. Kriegman. Visual tracking and recognition using probabilistic appearance manifolds. Computer Vision and Image Understanding, 99(3):303-331, 2005.

\begin{tabular}{|l|l|l|l|}
\hline \multicolumn{5}{|c|}{ PARTICIPANT KEY } \\
\hline $\mathrm{A}=$ DIGITAL BARRIERS & $\mathrm{E}=$ NEUROTECHNOLOGY & $\mathrm{I}=$ EYEDEA & $\mathrm{M}=$ NEC \\
\hline $\mathrm{B}=$ HBINNO & $\mathrm{F}=$ VAPPLICA & $\mathrm{J}=$ HISIGN & $\mathrm{N}=$ TOSHIBA \\
\hline $\mathrm{C}=$ VIGILANT & $\mathrm{G}=$ MORPHO & $\mathrm{K}=$ COGNITEC & $\mathrm{Q}=$ IMAGUS \\
\hline $\mathrm{D}=$ AYONIX & $\mathrm{H}=$ 3M COGENT & $\mathrm{L}=$ CYBEREXTRUDER & $\mathrm{R}=$ RANK ONE \\
\hline
\end{tabular}

\begin{tabular}{|l|l|l|l|l|l|}
\hline SET & SCENE & CAMERA & SET & SCENE & CAMERA \\
\hline C & PHOTOJOURNALISM & PRO & T & CONCOURSE & PRO \\
\hline J & PASSENGER LOADING & PRO & H & CONCOURSE & PRO \\
\hline P & SPORTS ARENA & CONSUMER & U & CHOKEPOINT & WEBCAM \\
\hline L & LUGGAGE RACK & WEBCAM & & & \\
\hline
\end{tabular}


[29] Joseph N. Onek and Sharon Bradford Franklin. Guidelines for public video surveillance - a guide to protecting communities and preserving civil liberties. Technical report, The Constitution Project. A report of TCP's Liberty and Security Committee, 1200 18th Street, NW Suite 1000 Washington DC, 20036, 2007. http://www.constitutionproject.org/wp-content/uploads/2012/09/54.pdf.

[30] O. M. Parkhi, A. Vedaldi, and A. Zisserman. Deep face recognition. In British Machine Vision Conference, 2015.

[31] Florian Schroff, Dmitry Kalenichenko, and James Philbin. Facenet: A unified embedding for face recognition and clustering. In The IEEE Conference on Computer Vision and Pattern Recognition (CVPR), June 2015.

[32] Mahmood Sharif, Sruti Bhagavatula, Lujo Bauer, and Michael K. Reiter. Accessorize to a crime: Real and stealthy attacks on state-of-the-art face recognition. In Proceedings of the 23rd ACM SIGSAC Conference on Computer and Communications Security, October 2016. http://dx.doi.org/10.1145/2976749.2978392.

[33] David White, James D. Dunn, Alexandra C. Schmid, and Richard I. Kemp. Error rates in users of automatic face recognition software. PLoS ONE, October 2015.

[34] Andreas Wolf. Reference facial images for mrtds - portrait quality. Technical report, International Civil Aviation Organization (ICAO), 2017. Technical Report.

[35] Galit Yovel and Alice J. OToole. Recognizing people in motion. Trends in Cognitive Sciences, 20(5):383-395, May 2016.

\begin{tabular}{|l|l|l|l|}
\hline \multicolumn{4}{|c|}{ PARTICIPANT KEY } \\
\hline A = DIGITAL BARRIERS & E = NEUROTECHNOLOGY & I = EYEDEA & $\mathrm{M}=$ NEC \\
\hline B = HBINNO & F = VAPPLICA & J = HISIGN & N $=$ TOSHIBA \\
\hline C = VIGILANT & G = MORPHO & K = COGNITEC & Q IMAGUS \\
\hline D = AYONIX & H = 3M COGENT & L = CYBEREXTRUDER & R = RANK ONE \\
\hline
\end{tabular}

\begin{tabular}{|l|l|l|l|l|l|}
\hline SET & SCENE & CAMERA & SET & SCENE & CAMERA \\
\hline C & PHOTOJOURNALISM & PRO & T & CONCOURSE & PRO \\
\hline J & PASSENGER LOADING & PRO & H & CONCOURSE & PRO \\
\hline P & SPORTS ARENA & CONSUMER & U & CHOKEPOINT & WEBCAM \\
\hline L & LUGGAGE RACK & WEBCAM & & & \\
\hline
\end{tabular}




\section{APPENDIX I: INTEROCULAR DISTANCES}

\begin{tabular}{|c|c|c|c|c|c|c|c|c|}
\hline DATASET P DOOR & \multicolumn{6}{|c|}{ VIDEO SEARCH } & \multirow{2}{*}{\multicolumn{2}{|c|}{$\begin{array}{l}\text { STILL IMAGE } \\
\text { IOD (PIXELS) }\end{array}$}} \\
\hline & NUMBER OF & MEAN TRACK & MEAN TRACK & MEAN & MEAN & MEAN & & \\
\hline ALGORITHM & TRACKS & LENGTH (FRAMES) & EXTENT (FRAMES) & MIN IOD (PX) & MEAN IOD (PX) & MAX IOD (PX) & MEAN & STD. DEV \\
\hline A30V & 24383 & 10 & 10 & 35 & 37 & 39 & 123 & 25 \\
\hline A31V & 24383 & 10 & 10 & 35 & 37 & 39 & 123 & 25 \\
\hline B30V & 6014 & 44 & 53 & 34 & 46 & 142 & 125 & 25 \\
\hline C30V & 18552 & 7 & 8 & 47 & 51 & 55 & 126 & 26 \\
\hline C31V & 19962 & 6 & 7 & 45 & 49 & 53 & 126 & 26 \\
\hline D30V & 22065 & 8 & 8 & 55 & 57 & 59 & 125 & 27 \\
\hline D31V & 8741 & 29 & 30 & 42 & 48 & 55 & 125 & 28 \\
\hline E30V & 4297 & 74 & 100 & 37 & 49 & 62 & 123 & 25 \\
\hline E31V & 4297 & 74 & 100 & 37 & 49 & 62 & 123 & 25 \\
\hline F30V & 35204 & 2 & 9 & 43 & 45 & 47 & 122 & 24 \\
\hline G30V & 2673 & 52 & 78 & 41 & 49 & 62 & 125 & 25 \\
\hline G31V & 3623 & 43 & 85 & 38 & 44 & 52 & 125 & 25 \\
\hline G32V & 3623 & 43 & 85 & 38 & 44 & 52 & 125 & 25 \\
\hline H30V & 5654 & 40 & 45 & 40 & 48 & 62 & 125 & 25 \\
\hline H31V & 5654 & 40 & 45 & 40 & 48 & 62 & 125 & 25 \\
\hline H32V & 5654 & 40 & 45 & 40 & 48 & 62 & 125 & 25 \\
\hline I30V & 7629 & 50 & 50 & 16 & 28 & 40 & 127 & 25 \\
\hline I31V & 7629 & 50 & 50 & 16 & 28 & 40 & 127 & 25 \\
\hline J30V & 2564 & 43 & 63 & 46 & 55 & 64 & 127 & 25 \\
\hline J31V & 2564 & 43 & 63 & 46 & 55 & 64 & 127 & 25 \\
\hline $\mathrm{J} 32 \mathrm{~V}$ & 2564 & 43 & 63 & 46 & 55 & 64 & 127 & 25 \\
\hline K30V & 34354 & 6 & 20 & 16 & 20 & 23 & 126 & 25 \\
\hline K31V & 5022 & 61 & 358 & 7 & 27 & 45 & 126 & 25 \\
\hline K32V & 2537 & 52 & 102 & 44 & 50 & 56 & 126 & 25 \\
\hline K33V & 1680 & 89 & 228 & 42 & 51 & 61 & 126 & 25 \\
\hline L30V & 6385 & 27 & 28 & 12 & 32 & 49 & 123 & 24 \\
\hline M30V & 6993 & 35 & 40 & 34 & 38 & 43 & 111 & 22 \\
\hline M31V & 6993 & 35 & 40 & 34 & 38 & 43 & 111 & 22 \\
\hline M32V & 6993 & 35 & 40 & 34 & 38 & 43 & 111 & 22 \\
\hline N30V & 4148 & 43 & 60 & 36 & 44 & 53 & 124 & 25 \\
\hline N31V & 4148 & 43 & 60 & 36 & 44 & 53 & 124 & 25 \\
\hline N32V & 4148 & 43 & 60 & 36 & 44 & 53 & 124 & 25 \\
\hline N33V & 4148 & 43 & 60 & 36 & 44 & 53 & 124 & 25 \\
\hline Q30V & 9750 & 27 & 29 & 30 & 37 & 44 & 121 & 24 \\
\hline Q31V & 9750 & 27 & 29 & 30 & 37 & 44 & 121 & 24 \\
\hline R30V & 6578 & 5 & 43 & 48 & 51 & 56 & 123 & 24 \\
\hline
\end{tabular}

Table 36: For DATASET P: SPORTS ARENA and each video processing algorithm the table shows: a) the number of reported tracks; b) the mean number of frames reported within those tracks; c) the mean extent (first minus last frames indices plus one); $d$ ) the mean over all tracks of of the minimum interocular distance (IOD) reported; e) the mean of the mean IOD; f) the mean of the maximum $I O D ; g)$ the enrollment still image mean IOD; and h) its standard deviation. For some algorithms (F, J, $L, R)$ the tracks don't include all consecutive frames, so the extent of the track can exceed the number of frames in it. For the $D, F$, and $L$ algorithms, the reported still-image eye coordinates are erroneous.

\begin{tabular}{|c|c|c|c|c|c|c|c|c|c|}
\hline \multicolumn{4}{|c|}{ PARTICIPANT KEY } & SET & SCENE & CAMERA & SET & SCENE & CAMERA \\
\hline $\mathrm{A}=$ DIGITAL BARRIERS & $\mathrm{E}=$ NEUROTECHNOLOGY & $\mathrm{I}=\mathrm{EYEDEA}$ & $\mathrm{M}=\mathrm{NEC}$ & C & PHOTOJOURNALISM & PRO & $\mathrm{T}$ & CONCOURSE & PRO \\
\hline $\mathrm{B}=\mathrm{HBINNO}$ & $\mathrm{F}=$ VAPPLICA & $\mathrm{J}=\mathrm{HISIGN}$ & $\mathrm{N}=$ TOSHIBA & $\mathrm{J}$ & PASSENGER LOADING & PRO & $\mathrm{H}$ & CONCOURSE & PRO \\
\hline $\mathrm{C}=$ VIGILANT & $\mathrm{G}=\mathrm{MORPHO}$ & $\mathrm{K}=$ COGNITEC & $\mathrm{Q}=\mathrm{IMAGUS}$ & $\mathrm{P}$ & SPORTS ARENA & CONSUMER & $\mathrm{U}$ & CHOKEPOINT & WEBCAM \\
\hline $\mathrm{D}=$ AYONIX & $\mathrm{H}=3 \mathrm{M}$ COGENT & $\mathrm{L}=$ CYBEREXTRUDER & $\mathrm{R}=\mathrm{RANK}$ ONE & $\mathrm{L}$ & LUGGAGE RACK & WEBCAM & & & \\
\hline
\end{tabular}




\begin{tabular}{|c|c|c|c|c|c|c|c|c|}
\hline DATASET P LOW NEAR & \multicolumn{6}{|c|}{ VIDEO SEARCH } & \multirow{2}{*}{\multicolumn{2}{|c|}{$\begin{array}{l}\text { STILL IMAGE } \\
\text { IOD (PIXELS) }\end{array}$}} \\
\hline & NUMBER OF & MEAN TRACK & MEAN TRACK & MEAN & MEAN & MEAN & & \\
\hline ALGORITHM & TRACKS & LENGTH (FRAMES) & EXTENT (FRAMES) & MIN IOD (PX) & MEAN IOD (PX) & MAX IOD (PX) & MEAN & STD. DEV \\
\hline A30V & 45875 & 11 & 11 & 42 & 45 & 48 & 123 & 25 \\
\hline A31V & 45875 & 11 & 11 & 42 & 45 & 48 & 123 & 25 \\
\hline $\mathrm{B} 30 \mathrm{~V}$ & 8117 & 77 & 96 & 40 & 55 & 178 & 125 & 25 \\
\hline $\mathrm{C} 30 \mathrm{~V}$ & 33188 & 9 & 10 & 55 & 59 & 64 & 126 & 26 \\
\hline C31V & 36176 & 9 & 10 & 52 & 56 & 61 & 126 & 26 \\
\hline D30V & 34092 & 11 & 11 & 60 & 63 & 65 & 125 & 27 \\
\hline D31V & 14196 & 35 & 35 & 47 & 55 & 63 & 125 & 28 \\
\hline E30V & 5697 & 95 & 129 & 37 & 47 & 55 & 123 & 25 \\
\hline E31V & 5697 & 95 & 129 & 37 & 47 & 55 & 123 & 25 \\
\hline F30V & 36430 & 5 & 32 & 59 & 62 & 66 & 122 & 24 \\
\hline G30V & 4793 & 83 & 116 & 45 & 55 & 69 & 125 & 25 \\
\hline G31V & 4403 & 106 & 201 & 38 & 47 & 57 & 125 & 25 \\
\hline G32V & 4403 & 106 & 201 & 38 & 47 & 57 & 125 & 25 \\
\hline H30V & 9148 & 55 & 60 & 47 & 58 & 74 & 125 & 25 \\
\hline H31V & 9148 & 55 & 60 & 47 & 58 & 74 & 125 & 25 \\
\hline H32V & 9148 & 55 & 60 & 47 & 58 & 74 & 125 & 25 \\
\hline $\mathrm{I} 30 \mathrm{~V}$ & 12869 & 62 & 62 & 15 & 31 & 47 & 127 & 25 \\
\hline I31V & 12869 & 62 & 62 & 15 & 31 & 47 & 127 & 25 \\
\hline J30V & 4021 & 74 & 98 & 53 & 66 & 77 & 127 & 25 \\
\hline $\mathrm{J} 31 \mathrm{~V}$ & 4021 & 74 & 98 & 53 & 66 & 77 & 127 & 25 \\
\hline $\mathrm{J} 32 \mathrm{~V}$ & 4021 & 74 & 98 & 53 & 66 & 77 & 127 & 25 \\
\hline K30V & 41675 & 12 & 31 & 15 & 23 & 27 & 126 & 25 \\
\hline K31V & 8820 & 73 & 344 & 9 & 33 & 50 & 126 & 25 \\
\hline K32V & 3694 & 102 & 225 & 50 & 56 & 62 & 126 & 25 \\
\hline K33V & 3629 & 115 & 279 & 50 & 57 & 65 & 126 & 25 \\
\hline L30V & 11966 & 33 & 34 & 18 & 40 & 59 & 123 & 24 \\
\hline M30V & 12700 & 46 & 52 & 36 & 42 & 47 & 111 & 22 \\
\hline M31V & 12700 & 46 & 52 & 36 & 42 & 47 & 111 & 22 \\
\hline M32V & 12700 & 46 & 52 & 36 & 42 & 47 & 111 & 22 \\
\hline N30V & 7733 & 63 & 79 & 42 & 52 & 61 & 124 & 25 \\
\hline N31V & 7733 & 63 & 79 & 42 & 52 & 61 & 124 & 25 \\
\hline N32V & 7733 & 63 & 79 & 42 & 52 & 61 & 124 & 25 \\
\hline N33V & 7733 & 63 & 79 & 42 & 52 & 61 & 124 & 25 \\
\hline Q30V & 16025 & 32 & 35 & 34 & 45 & 53 & 121 & 24 \\
\hline Q31V & 16025 & 32 & 35 & 34 & 45 & 53 & 121 & 24 \\
\hline R30V & 9614 & 4 & 59 & 59 & 65 & 71 & 123 & 24 \\
\hline
\end{tabular}

Table 37: For DATASET P: SPORTS ARENA and each video processing algorithm the table shows: a) the number of reported tracks; b) the mean number of frames reported within those tracks; $c$ ) the mean extent (first minus last frames indices plus one); $d$ ) the mean over all tracks of of the minimum interocular distance (IOD) reported; e) the mean of the mean IOD; $f$ ) the mean of the maximum $I O D ; g)$ the enrollment still image mean IOD; and h) its standard deviation. For some algorithms $(F, J, L, R)$ the tracks don't include all consecutive frames, so the extent of the track can exceed the number of frames in it. For the $D, F$, and $L$ algorithms, the reported still-image eye coordinates are erroneous.

\begin{tabular}{|c|c|c|c|c|c|c|c|c|c|}
\hline \multicolumn{4}{|c|}{ PARTICIPANT KEY } & \multirow{2}{*}{\begin{tabular}{|l|} 
SET \\
C \\
\end{tabular}} & \multirow{2}{*}{\begin{tabular}{|l|} 
SCENE \\
PHOTOJOURNALISM \\
\end{tabular}} & \multirow{2}{*}{\begin{tabular}{|l} 
CAMERA \\
PRO
\end{tabular}} & \multirow{2}{*}{\begin{tabular}{|l|} 
SET \\
T
\end{tabular}} & \multirow{2}{*}{\begin{tabular}{|l|} 
SCENE \\
CONCOURSE
\end{tabular}} & \multirow{2}{*}{\begin{tabular}{|l|} 
CAMERA \\
PRO
\end{tabular}} \\
\hline $\mathrm{A}=$ DIGITAL BARRIERS & $\mathrm{E}=$ NEUROTECHNOLOGY & $\mathrm{I}=$ EYEDEA & $M=N E C$ & & & & & & \\
\hline $\mathrm{B}=\mathrm{HBINNO}$ & $\mathrm{F}=$ VAPPLICA & $\mathrm{J}=\mathrm{HISIGN}$ & $\mathrm{N}=$ TOSHIBA & $\mathrm{J}$ & PASSENGER LOADING & PRO & $\mathrm{H}$ & CONCOURSE & PRO \\
\hline $\mathrm{C}=$ VIGILANT & $\mathrm{G}=\mathrm{MORPHO}$ & $\mathrm{K}=$ COGNITEC & $\mathrm{Q}=\mathrm{IMAGUS}$ & $\mathrm{P}$ & SPORTS ARENA & CONSUMER & $\mathrm{U}$ & CHOKEPOINT & WEBCAM \\
\hline $\mathrm{D}=\mathrm{AYONIX}$ & $\mathrm{H}=3 \mathrm{M}$ COGENT & $\mathrm{L}=$ CYBEREXTRUDER & $\mathrm{R}=\mathrm{RANK}$ ONE & $\mathrm{L}$ & LUGGAGE RACK & WEBCAM & & & \\
\hline
\end{tabular}




\begin{tabular}{|c|c|c|c|c|c|c|c|c|}
\hline \multirow[t]{2}{*}{ DATASET P HIGH NEAR } & \multicolumn{6}{|c|}{ VIDEO SEARCH } & \multirow{2}{*}{\multicolumn{2}{|c|}{$\begin{array}{l}\text { STILL IMAGE } \\
\text { IOD (PIXELS) }\end{array}$}} \\
\hline & NUMBER OF & MEAN TRACK & MEAN TRACK & MEAN & MEAN & MEAN & & \\
\hline ALGORITHM & TRACKS & LENGTH (FRAMES) & EXTENT (FRAMES) & MIN IOD (PX) & MEAN IOD (PX) & MAX IOD (PX) & MEAN & STD. DEV \\
\hline A30V & 21953 & 6 & 6 & 39 & 41 & 42 & 123 & 25 \\
\hline A31V & 21953 & 6 & 6 & 39 & 41 & 42 & 123 & 25 \\
\hline B30V & 3353 & 70 & 99 & 40 & 61 & 269 & 125 & 25 \\
\hline C30V & 7852 & 7 & 8 & 56 & 60 & 64 & 126 & 26 \\
\hline C31V & 8072 & 6 & 8 & 55 & 59 & 63 & 126 & 26 \\
\hline D30V & 23794 & 6 & 6 & 63 & 64 & 66 & 125 & 27 \\
\hline D31V & 7954 & 22 & 23 & 50 & 56 & 63 & 125 & 28 \\
\hline E30V & 1919 & 95 & 127 & 43 & 52 & 63 & 123 & 25 \\
\hline E31V & 1919 & 95 & 127 & 43 & 52 & 63 & 123 & 25 \\
\hline F30V & 9980 & 4 & 23 & 55 & 57 & 58 & 122 & 24 \\
\hline G30V & 1125 & 97 & 164 & 48 & 58 & 76 & 125 & 25 \\
\hline G31V & 1538 & 68 & 165 & 44 & 51 & 60 & 125 & 25 \\
\hline G32V & 1538 & 68 & 165 & 44 & 51 & 60 & 125 & 25 \\
\hline H30V & 3700 & 43 & 50 & 51 & 61 & 79 & 125 & 25 \\
\hline H31V & 3700 & 43 & 50 & 51 & 61 & 79 & 125 & 25 \\
\hline H32V & 3700 & 43 & 50 & 51 & 61 & 79 & 125 & 25 \\
\hline I31V & 4529 & 60 & 60 & 15 & 28 & 44 & 127 & 25 \\
\hline J30V & 1618 & 54 & 86 & 52 & 60 & 68 & 127 & 25 \\
\hline J31V & 1618 & 54 & 86 & 52 & 60 & 68 & 127 & 25 \\
\hline $\mathrm{J} 32 \mathrm{~V}$ & 1618 & 54 & 86 & 52 & 60 & 68 & 127 & 25 \\
\hline K30V & 28308 & 5 & 22 & 12 & 16 & 19 & 126 & 25 \\
\hline K31V & 5128 & 42 & 353 & 6 & 24 & 40 & 126 & 25 \\
\hline K32V & 979 & 107 & 229 & 48 & 55 & 61 & 126 & 25 \\
\hline K33V & 984 & 135 & 323 & 46 & 54 & 62 & 126 & 25 \\
\hline L30V & 3034 & 26 & 27 & 15 & 31 & 57 & 123 & 24 \\
\hline M30V & 4216 & 36 & 44 & 44 & 48 & 53 & 111 & 22 \\
\hline M31V & 4216 & 36 & 44 & 44 & 48 & 53 & 111 & 22 \\
\hline M32V & 4216 & 36 & 44 & 44 & 48 & 53 & 111 & 22 \\
\hline N30V & 2064 & 55 & 89 & 44 & 53 & 62 & 124 & 25 \\
\hline N31V & 2064 & 55 & 89 & 44 & 53 & 62 & 124 & 25 \\
\hline N32V & 2064 & 55 & 89 & 44 & 53 & 62 & 124 & 25 \\
\hline N33V & 2064 & 55 & 89 & 44 & 53 & 62 & 124 & 25 \\
\hline Q30V & 10421 & 19 & 23 & 30 & 37 & 42 & 121 & 24 \\
\hline Q31V & 10421 & 19 & 23 & 30 & 37 & 42 & 121 & 24 \\
\hline
\end{tabular}

Table 38: For DATASET P: SPORTS ARENA and each video processing algorithm the table shows: a) the number of reported tracks; $b$ ) the mean number of frames reported within those tracks; $c$ ) the mean extent (first minus last frames indices plus one); $d)$ the mean over all tracks of of the minimum interocular distance (IOD) reported; e) the mean of the mean IOD; $f$ ) the mean of the maximum $I O D ; g)$ the enrollment still image mean IOD; and h) its standard deviation. For some algorithms $(F, J, L, R)$ the tracks don't include all consecutive frames, so the extent of the track can exceed the number of frames in it. For the $D, F$, and $L$ algorithms, the reported still-image eye coordinates are erroneous.

\begin{tabular}{|c|c|c|c|c|c|c|c|c|c|}
\hline \multicolumn{4}{|c|}{ PARTICIPANT KEY } & \multirow{2}{*}{\begin{tabular}{|l|} 
SET \\
C \\
\end{tabular}} & \multirow{2}{*}{\begin{tabular}{|l|} 
SCENE \\
PHOTOJOURNALISM \\
\end{tabular}} & \multirow{2}{*}{\begin{tabular}{|l} 
CAMERA \\
PRO
\end{tabular}} & \multirow{2}{*}{\begin{tabular}{|l|} 
SET \\
T
\end{tabular}} & \multirow{2}{*}{\begin{tabular}{|l|} 
SCENE \\
CONCOURSE
\end{tabular}} & \multirow{2}{*}{\begin{tabular}{|l|} 
CAMERA \\
PRO
\end{tabular}} \\
\hline $\mathrm{A}=$ DIGITAL BARRIERS & $\mathrm{E}=$ NEUROTECHNOLOGY & $\mathrm{I}=$ EYEDEA & $M=N E C$ & & & & & & \\
\hline $\mathrm{B}=\mathrm{HBINNO}$ & $\mathrm{F}=$ VAPPLICA & $\mathrm{J}=\mathrm{HISIGN}$ & $\mathrm{N}=$ TOSHIBA & $\mathrm{J}$ & PASSENGER LOADING & PRO & $\mathrm{H}$ & CONCOURSE & PRO \\
\hline $\mathrm{C}=$ VIGILANT & $\mathrm{G}=\mathrm{MORPHO}$ & $\mathrm{K}=$ COGNITEC & $\mathrm{Q}=\mathrm{IMAGUS}$ & $\mathrm{P}$ & SPORTS ARENA & CONSUMER & $\mathrm{U}$ & CHOKEPOINT & WEBCAM \\
\hline $\mathrm{D}=\mathrm{AYONIX}$ & $\mathrm{H}=3 \mathrm{M}$ COGENT & $\mathrm{L}=$ CYBEREXTRUDER & $\mathrm{R}=\mathrm{RANK}$ ONE & $\mathrm{L}$ & LUGGAGE RACK & WEBCAM & & & \\
\hline
\end{tabular}




\begin{tabular}{|c|c|c|c|c|c|c|c|c|}
\hline DATASET P LOW FAR & \multicolumn{6}{|c|}{ VIDEO SEARCH } & \multirow{2}{*}{\multicolumn{2}{|c|}{$\begin{array}{l}\text { STILL IMAGE } \\
\text { IOD (PIXELS) }\end{array}$}} \\
\hline & NUMBER OF & MEAN TRACK & MEAN TRACK & MEAN & MEAN & MEAN & & \\
\hline ALGORITHM & TRACKS & LENGTH (FRAMES) & EXTENT (FRAMES) & MIN IOD (PX) & MEAN IOD (PX) & MAX IOD (PX) & MEAN & STD. DEV \\
\hline A30V & 65205 & 5 & 5 & 27 & 28 & 30 & 123 & 25 \\
\hline A31V & 65205 & 5 & 5 & 27 & 28 & 30 & 123 & 25 \\
\hline B30V & 10230 & 24 & 34 & 32 & 43 & 125 & 125 & 25 \\
\hline C30V & 23004 & 4 & 5 & 37 & 40 & 42 & 126 & 26 \\
\hline C31V & 32672 & 4 & 5 & 31 & 34 & 36 & 126 & 26 \\
\hline D30V & 24399 & 5 & 5 & 60 & 63 & 66 & 125 & 27 \\
\hline D31V & 12360 & 15 & 16 & 44 & 50 & 57 & 125 & 28 \\
\hline E30V & 8802 & 41 & 63 & 24 & 29 & 36 & 123 & 25 \\
\hline E31V & 8802 & 41 & 63 & 24 & 29 & 36 & 123 & 25 \\
\hline F30V & 27367 & 2 & 5 & 41 & 42 & 44 & 122 & 24 \\
\hline G30V & 3023 & 23 & 38 & 39 & 45 & 54 & 125 & 25 \\
\hline G31V & 9440 & 16 & 43 & 28 & 31 & 36 & 125 & 25 \\
\hline G32V & 9440 & 16 & 43 & 28 & 31 & 36 & 125 & 25 \\
\hline H30V & 9088 & 23 & 28 & 36 & 42 & 51 & 125 & 25 \\
\hline H31V & 9088 & 23 & 28 & 36 & 42 & 51 & 125 & 25 \\
\hline H32V & 9088 & 23 & 28 & 36 & 42 & 51 & 125 & 25 \\
\hline I30V & 19650 & 34 & 34 & 12 & 18 & 25 & 127 & 25 \\
\hline I31V & 19650 & 34 & 34 & 12 & 18 & 25 & 127 & 25 \\
\hline J30V & 3178 & 20 & 31 & 48 & 53 & 61 & 127 & 25 \\
\hline J31V & 3178 & 20 & 31 & 48 & 53 & 61 & 127 & 25 \\
\hline $\mathrm{J} 32 \mathrm{~V}$ & 3178 & 20 & 31 & 48 & 53 & 61 & 127 & 25 \\
\hline K30V & 62966 & 3 & 18 & 15 & 18 & 20 & 126 & 25 \\
\hline K31V & 7744 & 53 & 332 & 5 & 23 & 38 & 126 & 25 \\
\hline K32V & 3488 & 29 & 72 & 32 & 36 & 39 & 126 & 25 \\
\hline K33V & 2211 & 59 & 226 & 29 & 36 & 44 & 126 & 25 \\
\hline L30V & 9640 & 17 & 18 & 6 & 21 & 38 & 123 & 24 \\
\hline M30V & 16696 & 19 & 24 & 24 & 26 & 29 & 111 & 22 \\
\hline M31V & 16696 & 19 & 24 & 24 & 26 & 29 & 111 & 22 \\
\hline M32V & 16696 & 19 & 24 & 24 & 26 & 29 & 111 & 22 \\
\hline N30V & 5924 & 26 & 40 & 34 & 41 & 49 & 124 & 25 \\
\hline N31V & 5924 & 26 & 40 & 34 & 41 & 49 & 124 & 25 \\
\hline N32V & 5924 & 26 & 40 & 34 & 41 & 49 & 124 & 25 \\
\hline N33V & 5924 & 26 & 40 & 34 & 41 & 49 & 124 & 25 \\
\hline Q30V & 34187 & 16 & 20 & 19 & 22 & 26 & 121 & 24 \\
\hline Q31V & 34187 & 16 & 20 & 19 & 22 & 26 & 121 & 24 \\
\hline R30V & 7128 & 3 & 41 & 65 & 70 & 77 & 123 & 24 \\
\hline
\end{tabular}

Table 39: For DATASET P: SPORTS ARENA and each video processing algorithm the table shows: a) the number of reported tracks; $b$ ) the mean number of frames reported within those tracks; $c$ ) the mean extent (first minus last frames indices plus one); $d$ ) the mean over all tracks of of the minimum interocular distance (IOD) reported; e) the mean of the mean IOD; $f$ ) the mean of the maximum $I O D ; g)$ the enrollment still image mean IOD; and h) its standard deviation. For some algorithms $(F, J, L, R)$ the tracks don't include all consecutive frames, so the extent of the track can exceed the number of frames in it. For the $D, F$, and $L$ algorithms, the reported still-image eye coordinates are erroneous.

\begin{tabular}{|c|c|c|c|c|c|c|c|c|c|}
\hline \multicolumn{4}{|c|}{ PARTICIPANT KEY } & SET & SCENE & CAMERA & SET & SCENE & CAMERA \\
\hline $\mathrm{A}=$ DIGITAL BARRIERS & $\mathrm{E}=$ NEUROTECHNOLOGY & $\mathrm{I}=$ EYEDEA & $\mathrm{M}=\mathrm{NEC}$ & C & PHOTOJOURNALISM & PRO & $\mathrm{T}$ & CONCOURSE & PRO \\
\hline $\mathrm{B}=\mathrm{HBINNO}$ & $\mathrm{F}=$ VAPPLICA & $\mathrm{J}=$ HISIGN & $\mathrm{N}=$ TOSHIBA & $\mathrm{J}$ & PASSENGER LOADING & PRO & $\mathrm{H}$ & CONCOURSE & PRO \\
\hline $\mathrm{C}=$ VIGILANT & $\mathrm{G}=\mathrm{MORPHO}$ & $\mathrm{K}=$ COGNITEC & $\mathrm{Q}=$ IMAGUS & $\mathrm{P}$ & SPORTS ARENA & CONSUMER & $\mathrm{U}$ & CHOKEPOINT & WEBCAM \\
\hline $\mathrm{D}=\mathrm{AYONIX}$ & $\mathrm{H}=3 \mathrm{M}$ COGENT & $\mathrm{L}=$ CYBEREXTRUDER & $\mathrm{R}=\mathrm{RANK}$ ONE & $\mathrm{L}$ & LUGGAGE RACK & WEBCAM & & & \\
\hline
\end{tabular}




\begin{tabular}{|c|c|c|c|c|c|c|c|c|}
\hline DATASET P HIGH FAR & \multicolumn{6}{|c|}{ VIDEO SEARCH } & \multirow{2}{*}{\multicolumn{2}{|c|}{$\begin{array}{l}\text { STILL IMAGE } \\
\text { IOD (PIXELS) }\end{array}$}} \\
\hline & NUMBER OF & MEAN TRACK & MEAN TRACK & MEAN & MEAN & MEAN & & \\
\hline ALGORITHM & TRACKS & LENGTH (FRAMES) & EXTENT (FRAMES) & MIN IOD (PX) & MEAN IOD (PX) & MAX IOD (PX) & MEAN & STD. DEV \\
\hline A30V & 92654 & 6 & 6 & 22 & 23 & 24 & 123 & 25 \\
\hline A31V & 92654 & 6 & 6 & 22 & 23 & 24 & 123 & 25 \\
\hline B30V & 15079 & 24 & 32 & 28 & 36 & 96 & 125 & 25 \\
\hline C30V & 40243 & 4 & 5 & 30 & 33 & 35 & 126 & 26 \\
\hline C31V & 60916 & 5 & 6 & 25 & 28 & 30 & 126 & 26 \\
\hline D30V & 29462 & 4 & 4 & 60 & 62 & 63 & 125 & 27 \\
\hline D31V & 15773 & 16 & 18 & 38 & 42 & 47 & 125 & 28 \\
\hline E30V & 13747 & 57 & 83 & 21 & 25 & 31 & 123 & 25 \\
\hline E31V & 13747 & 57 & 83 & 21 & 25 & 31 & 123 & 25 \\
\hline F30V & 40336 & 2 & 4 & 36 & 38 & 39 & 122 & 24 \\
\hline G30V & 4209 & 22 & 43 & 32 & 37 & 46 & 125 & 25 \\
\hline G31V & 12635 & 23 & 58 & 25 & 28 & 33 & 125 & 25 \\
\hline G32V & 12635 & 23 & 58 & 25 & 28 & 33 & 125 & 25 \\
\hline H30V & 11421 & 32 & 39 & 30 & 35 & 45 & 125 & 25 \\
\hline H31V & 11421 & 32 & 39 & 30 & 35 & 45 & 125 & 25 \\
\hline H32V & 11421 & 32 & 39 & 30 & 35 & 45 & 125 & 25 \\
\hline I30V & 32034 & 42 & 42 & 9 & 15 & 20 & 127 & 25 \\
\hline I31V & 32034 & 42 & 42 & 9 & 15 & 20 & 127 & 25 \\
\hline J30V & 2937 & 22 & 36 & 41 & 46 & 53 & 127 & 25 \\
\hline J31V & 2937 & 22 & 36 & 41 & 46 & 53 & 127 & 25 \\
\hline $\mathrm{J} 32 \mathrm{~V}$ & 2937 & 22 & 36 & 41 & 46 & 53 & 127 & 25 \\
\hline K30V & 73715 & 6 & 20 & 13 & 18 & 21 & 126 & 25 \\
\hline K31V & 13124 & 58 & 217 & 2 & 18 & 31 & 126 & 25 \\
\hline K32V & 6657 & 32 & 81 & 24 & 27 & 30 & 126 & 25 \\
\hline K33V & 4198 & 69 & 266 & 21 & 26 & 33 & 126 & 25 \\
\hline L30V & 14776 & 19 & 21 & 5 & 19 & 33 & 123 & 24 \\
\hline M30V & 29021 & 24 & 30 & 19 & 21 & 24 & 111 & 22 \\
\hline M31V & 29021 & 24 & 30 & 19 & 21 & 24 & 111 & 22 \\
\hline M32V & 29021 & 24 & 30 & 19 & 21 & 24 & 111 & 22 \\
\hline N30V & 7108 & 32 & 52 & 27 & 33 & 39 & 124 & 25 \\
\hline N31V & 7108 & 32 & 52 & 27 & 33 & 39 & 124 & 25 \\
\hline N32V & 7108 & 32 & 52 & 27 & 33 & 39 & 124 & 25 \\
\hline N33V & 7108 & 32 & 52 & 27 & 33 & 39 & 124 & 25 \\
\hline Q30V & 34001 & 23 & 26 & 20 & 23 & 27 & 121 & 24 \\
\hline Q31V & 34001 & 23 & 26 & 20 & 23 & 27 & 121 & 24 \\
\hline R30V & 8551 & 3 & 33 & 43 & 45 & 50 & 123 & 24 \\
\hline
\end{tabular}

Table 40: For DATASET P: SPORTS ARENA and each video processing algorithm the table shows: a) the number of reported tracks; b) the mean number of frames reported within those tracks; $c$ ) the mean extent (first minus last frames indices plus one); $d$ ) the mean over all tracks of of the minimum interocular distance (IOD) reported; e) the mean of the mean IOD; $f$ ) the mean of the maximum $I O D ; g)$ the enrollment still image mean IOD; and h) its standard deviation. For some algorithms $(F, J, L, R)$ the tracks don't include all consecutive frames, so the extent of the track can exceed the number of frames in it. For the $D, F$, and $L$ algorithms, the reported still-image eye coordinates are erroneous.

\begin{tabular}{|l|l|l|l|}
\hline \multicolumn{4}{|c|}{ PARTICIPANT KEY } \\
\hline $\mathrm{A}=$ DIGITAL BARRIERS & $\mathrm{E}=$ NEUROTECHNOLOGY & $\mathrm{I}=$ EYEDEA & $\mathrm{M}=$ NEC \\
\hline $\mathrm{B}=$ HBINNO & $\mathrm{F}=$ VAPPLICA & $\mathrm{J}=$ HISIGN & $\mathrm{N}=$ TOSHIBA \\
\hline $\mathrm{C}=$ VIGILANT & $\mathrm{G}=$ MORPHO & $\mathrm{K}=$ COGNITEC & $\mathrm{Q}=$ IMAGUS \\
\hline $\mathrm{D}=$ AYONIX & $\mathrm{H}=$ 3M COGENT & $\mathrm{L}=$ CYBEREXTRUDER & $\mathrm{R}=$ RANK ONE \\
\hline
\end{tabular}

\begin{tabular}{|l|l|l|l|l|l|}
\hline SET & SCENE & CAMERA & SET & SCENE & CAMERA \\
\hline C & PHOTOJOURNALISM & PRO & T & CONCOURSE & PRO \\
\hline J & PASSENGER LOADING & PRO & H & CONCOURSE & PRO \\
\hline P & SPORTS ARENA & CONSUMER & U & CHOKEPOINT & WEBCAM \\
\hline L & LUGGAGE RACK & WEBCAM & & & \\
\hline
\end{tabular}




\begin{tabular}{|c|c|c|c|c|c|c|c|c|}
\hline DATASET H F & \multicolumn{6}{|c|}{ VIDEO SEARCH } & \multirow{2}{*}{\multicolumn{2}{|c|}{$\begin{array}{l}\text { STILL IMAGE } \\
\text { IOD (PIXELS) }\end{array}$}} \\
\hline & NUMBER OF & MEAN TRACK & MEAN TRACK & MEAN & MEAN & MEAN & & \\
\hline ALGORITHM & TRACKS & LENGTH (FRAMES) & EXTENT (FRAMES) & MIN IOD (PX) & MEAN IOD (PX) & MAX IOD (PX) & MEAN & STD. DEV \\
\hline $\mathrm{A} 30 \mathrm{~V}$ & 251 & 11 & 11 & 35 & 38 & 41 & 123 & 30 \\
\hline A31V & 251 & 11 & 11 & 35 & 38 & 41 & 123 & 30 \\
\hline B30V & 51 & 73 & 77 & 40 & 71 & 485 & 125 & 30 \\
\hline $\mathrm{C} 30 \mathrm{~V}$ & 205 & 9 & 10 & 50 & 53 & 57 & 127 & 32 \\
\hline C31V & 207 & 9 & 10 & 49 & 53 & 57 & 127 & 32 \\
\hline D30V & 192 & 15 & 15 & 51 & 55 & 60 & 126 & 36 \\
\hline D31V & 66 & 50 & 51 & 42 & 54 & 67 & 126 & 36 \\
\hline E30V & 41 & 81 & 92 & 39 & 55 & 78 & 123 & 31 \\
\hline E31V & 41 & 81 & 92 & 39 & 55 & 78 & 123 & 31 \\
\hline F30V & 283 & 5 & 28 & 51 & 53 & 56 & 121 & 30 \\
\hline G30V & 34 & 55 & 86 & 41 & 56 & 82 & 125 & 31 \\
\hline G31V & 36 & 73 & 297 & 37 & 49 & 70 & 125 & 31 \\
\hline G32V & 36 & 73 & 297 & 37 & 49 & 70 & 125 & 31 \\
\hline H30V & 70 & 48 & 53 & 62 & 80 & 108 & 126 & 31 \\
\hline H31V & 70 & 48 & 53 & 62 & 80 & 108 & 126 & 31 \\
\hline H32V & 70 & 48 & 53 & 62 & 80 & 108 & 126 & 31 \\
\hline $\mathrm{I} 30 \mathrm{~V}$ & 62 & 74 & 74 & 22 & 43 & 64 & 126 & 31 \\
\hline I31V & 62 & 74 & 74 & 22 & 43 & 64 & 126 & 31 \\
\hline $\mathrm{J} 30 \mathrm{~V}$ & 43 & 57 & 68 & 49 & 63 & 82 & 127 & 32 \\
\hline J31V & 43 & 57 & 68 & 49 & 63 & 82 & 127 & 32 \\
\hline $\mathrm{J} 32 \mathrm{~V}$ & 43 & 57 & 68 & 49 & 63 & 82 & 127 & 32 \\
\hline K30V & 917 & 4 & 189 & 15 & 17 & 18 & 127 & 31 \\
\hline K31V & 59 & 87 & 2460 & 10 & 28 & 52 & 127 & 31 \\
\hline K32V & 45 & 51 & 163 & 43 & 52 & 65 & 127 & 31 \\
\hline K33V & 36 & 64 & 302 & 43 & 54 & 71 & 127 & 31 \\
\hline L30V & 63 & 35 & 37 & 21 & 47 & 66 & 123 & 30 \\
\hline M30V & 56 & 60 & 66 & 36 & 47 & 60 & 111 & 28 \\
\hline M31V & 56 & 60 & 66 & 36 & 47 & 60 & 111 & 28 \\
\hline M32V & 56 & 60 & 66 & 36 & 47 & 60 & 111 & 28 \\
\hline N30V & 52 & 56 & 68 & 38 & 53 & 71 & 125 & 31 \\
\hline N31V & 52 & 56 & 68 & 38 & 53 & 71 & 125 & 31 \\
\hline N32V & 52 & 56 & 68 & 38 & 53 & 71 & 125 & 31 \\
\hline N33V & 52 & 56 & 68 & 38 & 53 & 71 & 125 & 31 \\
\hline Q30V & 72 & 40 & 41 & 39 & 50 & 61 & 122 & 30 \\
\hline Q31V & 72 & 40 & 41 & 39 & 50 & 61 & 122 & 30 \\
\hline R30V & 120 & 2 & 96 & 47 & 51 & 56 & 123 & 30 \\
\hline
\end{tabular}

Table 41: For DATASET H: TRAVEL WALKWAY and each video processing algorithm the table shows: a) the number of reported tracks; b) the mean number of frames reported within those tracks; c) the mean extent (first minus last frames indices plus one); d) the mean over all tracks of of the minimum interocular distance (IOD) reported; e) the mean of the mean IOD; f) the mean of the maximum $I O D ; g)$ the enrollment still image mean IOD; and h) its standard deviation. For some algorithms $(F, J, L, R)$ the tracks don't include all consecutive frames, so the extent of the track can exceed the number of frames in it. For the $D, F$, and $L$ algorithms, the reported still-image eye coordinates are erroneous.

\begin{tabular}{|c|c|c|c|c|c|c|c|c|c|}
\hline \multicolumn{4}{|c|}{ PARTICIPANT KEY } & SET & SCENE & CAMERA & SET & SCENE & CAMERA \\
\hline $\mathrm{A}=$ DIGITAL BARRIERS & $\mathrm{E}=$ NEUROTECHNOLOGY & $\mathrm{I}=$ EYEDEA & $\mathrm{M}=\mathrm{NEC}$ & C & PHOTOJOURNALISM & PRO & $\mathrm{T}$ & CONCOURSE & PRO \\
\hline $\mathrm{B}=\mathrm{HBINNO}$ & $\mathrm{F}=$ VAPPLICA & $\mathrm{J}=$ HISIGN & $\mathrm{N}=$ TOSHIBA & $\mathrm{J}$ & PASSENGER LOADING & PRO & $\mathrm{H}$ & CONCOURSE & PRO \\
\hline $\mathrm{C}=$ VIGILANT & $\mathrm{G}=\mathrm{MORPHO}$ & $\mathrm{K}=$ COGNITEC & $\mathrm{Q}=$ IMAGUS & $\mathrm{P}$ & SPORTS ARENA & CONSUMER & $\mathrm{U}$ & CHOKEPOINT & WEBCAM \\
\hline $\mathrm{D}=\mathrm{AYONIX}$ & $\mathrm{H}=3 \mathrm{M}$ COGENT & $\mathrm{L}=$ CYBEREXTRUDER & $\mathrm{R}=\mathrm{RANK}$ ONE & $\mathrm{L}$ & LUGGAGE RACK & WEBCAM & & & \\
\hline
\end{tabular}




\begin{tabular}{|c|c|c|c|c|c|c|c|c|}
\hline DATASET H R & \multicolumn{6}{|c|}{ VIDEO SEARCH } & \multirow{2}{*}{\multicolumn{2}{|c|}{$\begin{array}{l}\text { STILL IMAGE } \\
\text { IOD (PIXELS) }\end{array}$}} \\
\hline & NUMBER OF & MEAN TRACK & MEAN TRACK & MEAN & MEAN & MEAN & & \\
\hline ALGORITHM & TRACKS & LENGTH (FRAMES) & EXTENT (FRAMES) & MIN IOD (PX) & MEAN IOD (PX) & MAX IOD (PX) & MEAN & STD. DEV \\
\hline A30V & 2020 & 5 & 5 & 68 & 71 & 75 & 123 & 30 \\
\hline A31V & 2020 & 5 & 5 & 68 & 71 & 75 & 123 & 30 \\
\hline B30V & 170 & 61 & 70 & 45 & 80 & 427 & 125 & 30 \\
\hline C30V & 695 & 6 & 7 & 60 & 63 & 66 & 127 & 32 \\
\hline C31V & 696 & 6 & 7 & 60 & 63 & 66 & 127 & 32 \\
\hline D30V & 7118 & 2 & 2 & 50 & 52 & 54 & 126 & 36 \\
\hline D31V & 1354 & 36 & 31 & 42 & 49 & 57 & 126 & 36 \\
\hline E30V & 117 & 79 & 102 & 43 & 58 & 81 & 123 & 31 \\
\hline E31V & 117 & 79 & 102 & 43 & 58 & 81 & 123 & 31 \\
\hline F30V & 731 & 3 & 12 & 51 & 53 & 55 & 121 & 30 \\
\hline G30V & 117 & 47 & 76 & 51 & 67 & 92 & 125 & 31 \\
\hline G31V & 167 & 46 & 236 & 51 & 63 & 81 & 125 & 31 \\
\hline G32V & 167 & 46 & 236 & 51 & 63 & 81 & 125 & 31 \\
\hline H30V & 166 & 61 & 66 & 48 & 61 & 82 & 126 & 31 \\
\hline H31V & 166 & 61 & 66 & 48 & 61 & 82 & 126 & 31 \\
\hline H32V & 166 & 61 & 66 & 48 & 61 & 82 & 126 & 31 \\
\hline I30V & 182 & 72 & 72 & 29 & 51 & 73 & 126 & 31 \\
\hline I31V & 182 & 72 & 72 & 29 & 51 & 73 & 126 & 31 \\
\hline $\mathrm{J} 30 \mathrm{~V}$ & 141 & 52 & 62 & 52 & 65 & 83 & 127 & 32 \\
\hline J31V & 141 & 52 & 62 & 52 & 65 & 83 & 127 & 32 \\
\hline $\mathrm{J} 32 \mathrm{~V}$ & 141 & 52 & 62 & 52 & 65 & 83 & 127 & 32 \\
\hline K30V & 3334 & 3 & 196 & 13 & 15 & 18 & 127 & 31 \\
\hline K31V & 266 & 63 & 2062 & 7 & 32 & 56 & 127 & 31 \\
\hline K32V & 133 & 55 & 98 & 48 & 60 & 77 & 127 & 31 \\
\hline K33V & 101 & 81 & 371 & 46 & 62 & 86 & 127 & 31 \\
\hline L30V & 204 & 26 & 27 & 16 & 40 & 67 & 123 & 30 \\
\hline M30V & 181 & 54 & 59 & 41 & 51 & 64 & 111 & 28 \\
\hline M31V & 181 & 54 & 59 & 41 & 51 & 64 & 111 & 28 \\
\hline M32V & 181 & 54 & 59 & 41 & 51 & 64 & 111 & 28 \\
\hline N30V & 154 & 60 & 68 & 42 & 57 & 79 & 125 & 31 \\
\hline N31V & 154 & 60 & 68 & 42 & 57 & 79 & 125 & 31 \\
\hline N32V & 154 & 60 & 68 & 42 & 57 & 79 & 125 & 31 \\
\hline N33V & 154 & 60 & 68 & 42 & 57 & 79 & 125 & 31 \\
\hline Q30V & 695 & 29 & 34 & 33 & 40 & 46 & 122 & 30 \\
\hline Q31V & 695 & 29 & 34 & 33 & 40 & 46 & 122 & 30 \\
\hline R30V & 364 & 2 & 113 & 63 & 68 & 75 & 123 & 30 \\
\hline
\end{tabular}

Table 42: For DATASET H: TRAVEL WALKWAY and each video processing algorithm the table shows: a) the number of reported tracks; b) the mean number of frames reported within those tracks; c) the mean extent (first minus last frames indices plus one); d) the mean over all tracks of of the minimum interocular distance (IOD) reported; e) the mean of the mean IOD; f) the mean of the maximum $I O D ; g)$ the enrollment still image mean IOD; and h) its standard deviation. For some algorithms $(F, J, L, R)$ the tracks don't include all consecutive frames, so the extent of the track can exceed the number of frames in it. For the $D, F$, and $L$ algorithms, the reported still-image eye coordinates are erroneous.

\begin{tabular}{|l|l|l|l|}
\hline \multicolumn{4}{|c|}{ PARTICIPANT KEY } \\
\hline $\mathrm{A}=$ DIGITAL BARRIERS & $\mathrm{E}=$ NEUROTECHNOLOGY & $\mathrm{I}=$ EYEDEA & $\mathrm{M}=$ NEC \\
\hline $\mathrm{B}=$ HBINNO & $\mathrm{F}=$ VAPPLICA & $\mathrm{J}=$ HISIGN & $\mathrm{N}=$ TOSHIBA \\
\hline $\mathrm{C}=$ VIGILANT & $\mathrm{G}=$ MORPHO & $\mathrm{K}=$ COGNITEC & $\mathrm{Q}=$ IMAGUS \\
\hline $\mathrm{D}=$ AYONIX & $\mathrm{H}=$ 3M COGENT & $\mathrm{L}=$ CYBEREXTRUDER & $\mathrm{R}=$ RANK ONE \\
\hline
\end{tabular}

\begin{tabular}{|l|l|l|l|l|l|}
\hline SET & SCENE & CAMERA & SET & SCENE & CAMERA \\
\hline C & PHOTOJOURNALISM & PRO & T & CONCOURSE & PRO \\
\hline J & PASSENGER LOADING & PRO & H & CONCOURSE & PRO \\
\hline P & SPORTS ARENA & CONSUMER & U & CHOKEPOINT & WEBCAM \\
\hline L & LUGGAGE RACK & WEBCAM & & & \\
\hline
\end{tabular}




\begin{tabular}{|c|c|c|c|c|c|c|c|c|}
\hline DATASET H S & \multicolumn{6}{|c|}{ VIDEO SEARCH } & \multirow{2}{*}{\multicolumn{2}{|c|}{$\begin{array}{l}\text { STILL IMAGE } \\
\text { IOD (PIXELS) }\end{array}$}} \\
\hline & NUMBER OF & MEAN TRACK & MEAN TRACK & MEAN & MEAN & MEAN & & \\
\hline ALGORITHM & TRACKS & LENGTH (FRAMES) & EXTENT (FRAMES) & MIN IOD (PX) & MEAN IOD (PX) & MAX IOD (PX) & MEAN & STD. DEV \\
\hline $\mathrm{A} 30 \mathrm{~V}$ & 1155 & 13 & 13 & 59 & 63 & 67 & 123 & 30 \\
\hline A31V & 1155 & 13 & 13 & 59 & 63 & 67 & 123 & 30 \\
\hline B30V & 271 & 65 & 75 & 61 & 86 & 307 & 125 & 30 \\
\hline $\mathrm{C} 30 \mathrm{~V}$ & 1442 & 7 & 8 & 91 & 97 & 103 & 127 & 32 \\
\hline C31V & 1447 & 7 & 8 & 91 & 97 & 102 & 127 & 32 \\
\hline D30V & 1708 & 9 & 9 & 64 & 66 & 69 & 126 & 36 \\
\hline D31V & 524 & 32 & 33 & 54 & 63 & 72 & 126 & 36 \\
\hline E30V & 167 & 74 & 83 & 58 & 74 & 98 & 123 & 31 \\
\hline E31V & 167 & 74 & 83 & 58 & 74 & 98 & 123 & 31 \\
\hline F30V & 1516 & 6 & 24 & 66 & 69 & 73 & 121 & 30 \\
\hline G30V & 196 & 48 & 73 & 58 & 72 & 92 & 125 & 31 \\
\hline G31V & 175 & 83 & 301 & 53 & 67 & 85 & 125 & 31 \\
\hline G32V & 175 & 83 & 301 & 53 & 67 & 85 & 125 & 31 \\
\hline H30V & 257 & 62 & 66 & 60 & 73 & 93 & 126 & 31 \\
\hline H31V & 257 & 62 & 66 & 60 & 73 & 93 & 126 & 31 \\
\hline H32V & 257 & 62 & 66 & 60 & 73 & 93 & 126 & 31 \\
\hline $\mathrm{I} 30 \mathrm{~V}$ & 253 & 77 & 77 & 34 & 59 & 80 & 126 & 31 \\
\hline I31V & 253 & 77 & 77 & 34 & 59 & 80 & 126 & 31 \\
\hline $\mathrm{J} 30 \mathrm{~V}$ & 224 & 59 & 68 & 62 & 74 & 89 & 127 & 32 \\
\hline J31V & 224 & 59 & 68 & 62 & 74 & 89 & 127 & 32 \\
\hline $\mathrm{J} 32 \mathrm{~V}$ & 224 & 59 & 68 & 62 & 74 & 89 & 127 & 32 \\
\hline K30V & 4715 & 5 & 323 & 14 & 16 & 18 & 127 & 31 \\
\hline K31V & 218 & 124 & 2508 & 20 & 46 & 76 & 127 & 31 \\
\hline K32V & 209 & 74 & 150 & 57 & 71 & 87 & 127 & 31 \\
\hline K33V & 184 & 85 & 239 & 58 & 74 & 92 & 127 & 31 \\
\hline L30V & 329 & 34 & 36 & 38 & 62 & 80 & 123 & 30 \\
\hline M30V & 248 & 61 & 65 & 49 & 62 & 76 & 111 & 28 \\
\hline M31V & 248 & 61 & 65 & 49 & 62 & 76 & 111 & 28 \\
\hline M32V & 248 & 61 & 65 & 49 & 62 & 76 & 111 & 28 \\
\hline N30V & 262 & 53 & 58 & 56 & 70 & 87 & 125 & 31 \\
\hline N31V & 262 & 53 & 58 & 56 & 70 & 87 & 125 & 31 \\
\hline N32V & 262 & 53 & 58 & 56 & 70 & 87 & 125 & 31 \\
\hline N33V & 262 & 53 & 58 & 56 & 70 & 87 & 125 & 31 \\
\hline Q30V & 413 & 34 & 37 & 51 & 62 & 71 & 122 & 30 \\
\hline Q31V & 413 & 34 & 37 & 51 & 62 & 71 & 122 & 30 \\
\hline R30V & 537 & 2 & 74 & 63 & 66 & 71 & 123 & 30 \\
\hline
\end{tabular}

Table 43: For DATASET H: TRAVEL WALKWAY and each video processing algorithm the table shows: a) the number of reported tracks; b) the mean number of frames reported within those tracks; c) the mean extent (first minus last frames indices plus one); d) the mean over all tracks of of the minimum interocular distance (IOD) reported; e) the mean of the mean IOD; f) the mean of the maximum $I O D ; g)$ the enrollment still image mean IOD; and $h)$ its standard deviation. For some algorithms $(F, J, L, R)$ the tracks don't include all consecutive frames, so the extent of the track can exceed the number of frames in it. For the $D, F$, and $L$ algorithms, the reported still-image eye coordinates are erroneous.

\begin{tabular}{|c|c|c|c|c|c|c|c|c|c|}
\hline \multicolumn{4}{|c|}{ PARTICIPANT KEY } & SET & SCENE & CAMERA & SET & SCENE & CAMERA \\
\hline $\mathrm{A}=$ DIGITAL BARRIERS & $\mathrm{E}=$ NEUROTECHNOLOGY & $\mathrm{I}=$ EYEDEA & $\mathrm{M}=\mathrm{NEC}$ & C & PHOTOJOURNALISM & PRO & $\mathrm{T}$ & CONCOURSE & PRO \\
\hline $\mathrm{B}=\mathrm{HBINNO}$ & $\mathrm{F}=$ VAPPLICA & $\mathrm{J}=$ HISIGN & $\mathrm{N}=$ TOSHIBA & $\mathrm{J}$ & PASSENGER LOADING & PRO & $\mathrm{H}$ & CONCOURSE & PRO \\
\hline $\mathrm{C}=$ VIGILANT & $\mathrm{G}=\mathrm{MORPHO}$ & $\mathrm{K}=$ COGNITEC & $\mathrm{Q}=$ IMAGUS & $\mathrm{P}$ & SPORTS ARENA & CONSUMER & $\mathrm{U}$ & CHOKEPOINT & WEBCAM \\
\hline $\mathrm{D}=\mathrm{AYONIX}$ & $\mathrm{H}=3 \mathrm{M}$ COGENT & $\mathrm{L}=$ CYBEREXTRUDER & $\mathrm{R}=\mathrm{RANK}$ ONE & $\mathrm{L}$ & LUGGAGE RACK & WEBCAM & & & \\
\hline
\end{tabular}




\begin{tabular}{|c|c|c|c|c|c|c|c|c|}
\hline \multirow[t]{2}{*}{ DATASET C } & \multicolumn{6}{|c|}{ VIDEO SEARCH } & \multirow{2}{*}{\multicolumn{2}{|c|}{$\begin{array}{l}\text { STILL IMAGE } \\
\text { IOD (PIXELS) }\end{array}$}} \\
\hline & NUMBER OF & MEAN TRACK & MEAN TRACK & MEAN & MEAN & MEAN & & \\
\hline ALGORITHM & TRACKS & LENGTH (FRAMES) & EXTENT (FRAMES) & MIN IOD (PX) & MEAN IOD (PX) & MAX IOD (PX) & MEAN & STD. DEV \\
\hline A30V & 215513 & 18 & 18 & 35 & 37 & 39 & 55 & 79 \\
\hline A31V & 215513 & 18 & 18 & 35 & 37 & 39 & 55 & 79 \\
\hline $\mathrm{B} 30 \mathrm{~V}$ & 26477 & 149 & 179 & 34 & 45 & 145 & 97 & 92 \\
\hline C30V & 148870 & 15 & 17 & 45 & 48 & 52 & 56 & 81 \\
\hline C31V & 170834 & 15 & 17 & 41 & 44 & 47 & 56 & 81 \\
\hline D30V & 109836 & 19 & 19 & 55 & 57 & 59 & 5679 & 10451 \\
\hline D31V & 45934 & 60 & 61 & 44 & 50 & 55 & 6576 & 10734 \\
\hline E30V & 26789 & 161 & 187 & 30 & 36 & 42 & 88 & 87 \\
\hline E31V & 26771 & 160 & 186 & 30 & 36 & 42 & 88 & 87 \\
\hline F30V & 253765 & 9 & 84 & 35 & 37 & 39 & 3502 & 8332 \\
\hline G30V & 20905 & 148 & 179 & 40 & 48 & 59 & 58 & 85 \\
\hline G31V & 20831 & 171 & 250 & 35 & 41 & 50 & 62 & 81 \\
\hline G32V & 20731 & 172 & 250 & 35 & 41 & 50 & 62 & 81 \\
\hline H30V & 40428 & 84 & 89 & 37 & 44 & 54 & 64 & 85 \\
\hline H31V & 40428 & 84 & 89 & 37 & 44 & 54 & 64 & 85 \\
\hline H32V & 40428 & 84 & 89 & 37 & 44 & 54 & 64 & 85 \\
\hline I30V & 65856 & 95 & 95 & 14 & 23 & 31 & 51 & 79 \\
\hline I31V & 65856 & 95 & 95 & 14 & 23 & 31 & 51 & 79 \\
\hline $\mathrm{J} 30 \mathrm{~V}$ & 21972 & 122 & 151 & 44 & 52 & 58 & 53 & 86 \\
\hline $\mathrm{J} 31 \mathrm{~V}$ & 21972 & 122 & 151 & 44 & 52 & 58 & 53 & 86 \\
\hline $\mathrm{J} 32 \mathrm{~V}$ & 21972 & 122 & 151 & 44 & 52 & 58 & 61 & 87 \\
\hline K30V & 109509 & 43 & 87 & 16 & 26 & 33 & 67 & 68 \\
\hline K31V & 41140 & 127 & 326 & 12 & 28 & 39 & 68 & 69 \\
\hline K32V & 26747 & 155 & 266 & 32 & 36 & 40 & 67 & 67 \\
\hline K33V & 24674 & 184 & 363 & 31 & 36 & 41 & 68 & 69 \\
\hline L30V & 80041 & 42 & 44 & 19 & 29 & 40 & 1226 & 5345 \\
\hline M30V & 60017 & 81 & 87 & 26 & 29 & 33 & 50 & 64 \\
\hline M31V & 60017 & 81 & 87 & 26 & 29 & 33 & 50 & 64 \\
\hline M32V & 60017 & 81 & 87 & 26 & 29 & 33 & 50 & 64 \\
\hline N30V & 25403 & 112 & 135 & 37 & 45 & 53 & 81 & 81 \\
\hline N31V & 25403 & 112 & 135 & 37 & 45 & 53 & 81 & 81 \\
\hline N32V & 25403 & 112 & 135 & 37 & 45 & 53 & 81 & 81 \\
\hline N33V & 25403 & 112 & 135 & 37 & 45 & 53 & 81 & 81 \\
\hline Q30V & 62617 & 46 & 48 & 29 & 36 & 41 & 36 & 64 \\
\hline Q31V & 62617 & 46 & 48 & 29 & 36 & 41 & 36 & 64 \\
\hline R30V & 55916 & 4 & 86 & 40 & 43 & 47 & 55 & 82 \\
\hline
\end{tabular}

Table 44: For DATASET C: PHOTOJOURNALISM and each video processing algorithm the table shows: a) the number of reported tracks; b) the mean number of frames reported within those tracks; c) the mean extent (first minus last frames indices plus one); d) the mean over all tracks of of the minimum interocular distance (IOD) reported; e) the mean of the mean IOD; f) the mean of the maximum $I O D ; g)$ the enrollment still image mean IOD; and h) its standard deviation. For some algorithms $(F, J, L, R)$ the tracks don't include all consecutive frames, so the extent of the track can exceed the number of frames in it. For the $D, F$, and $L$ algorithms, the reported still-image eye coordinates are erroneous.

\begin{tabular}{|c|c|c|c|c|c|c|c|c|c|}
\hline \multicolumn{4}{|c|}{ PARTICIPANT KEY } & SET & SCENE & CAMERA & SET & SCENE & CAMERA \\
\hline $\mathrm{A}=$ DIGITAL BARRIERS & $\mathrm{E}=$ NEUROTECHNOLOGY & $\mathrm{I}=$ EYEDEA & $\mathrm{M}=\mathrm{NEC}$ & C & PHOTOJOURNALISM & PRO & $\mathrm{T}$ & CONCOURSE & PRO \\
\hline $\mathrm{B}=\mathrm{HBINNO}$ & $\mathrm{F}=$ VAPPLICA & $\mathrm{J}=$ HISIGN & $\mathrm{N}=$ TOSHIBA & $\mathrm{J}$ & PASSENGER LOADING & PRO & $\mathrm{H}$ & CONCOURSE & PRO \\
\hline $\mathrm{C}=$ VIGILANT & $\mathrm{G}=\mathrm{MORPHO}$ & $\mathrm{K}=$ COGNITEC & $\mathrm{Q}=$ IMAGUS & $\mathrm{P}$ & SPORTS ARENA & CONSUMER & $\mathrm{U}$ & CHOKEPOINT & WEBCAM \\
\hline $\mathrm{D}=\mathrm{AYONIX}$ & $\mathrm{H}=3 \mathrm{M}$ COGENT & $\mathrm{L}=$ CYBEREXTRUDER & $\mathrm{R}=\mathrm{RANK}$ ONE & $\mathrm{L}$ & LUGGAGE RACK & WEBCAM & & & \\
\hline
\end{tabular}

